\title{
In vitro and in vivo characterization of the E3 ubiquitin ligase RNF157 in the brain
}

\author{
Dissertation \\ for the award of the degree \\ "Doctor rerum naturalium" \\ of the Georg-August-Universität Göttingen \\ within the doctoral program \\ Molecular Physiology of the Brain \\ of the Georg-August University School of Science (GAUSS)
}

submitted by

\section{Shih-Ju Lee}

born in Chiayi, Taiwan

Göttingen 2014 


\section{Thesis Committee Members:}

\section{Dr. Judith Stegmüller}

Laboratory of Cellular and Molecular Neurobiology,

Max-Planck-Institute of experimental Medicine, Göttingen

\section{Prof. Dr. Nils Brose}

Department of Molecular Neurobiology,

Max-Planck-Institute of experimental Medicine, Göttingen

\section{Prof. Dr. André Fischer}

Department for Psychiatry and Psychotherapy

German Center for Neurodegenerative Diseases (DZNE), Göttingen

\section{Extended thesis committee members:}

Reviewer: Dr. Judith Stegmüller, Laboratory of Cellular and Molecular Neurobiology, MaxPlanck-Institute of experimental Medicine, Göttingen

Reviewer: Prof. Dr. Nils Brose, Department of Molecular Neurobiology, Max-Planck-Institute of experimental Medicine, Göttingen

Prof. Dr. André Fischer, Department for Psychiatry and Psychotherapy German Center for Neurodegenerative Diseases (DZNE), Göttingen

Prof. Dr. Dr. Hannelore Ehrenreich, Division of Clinical Neuroscience, Max-Planck-Institute of experimental Medicine, Göttingen

Prof. Dr. Thomas Bayer, Division of Molecular Psychiatry, Georg-August-University Göttingen

Prof. Dr. Ralf Heinrich, Department of Cellular Neurobiology, Johann-Friedrich-BlumenbachSchwann-Schleiden Research Centre, Göttingen

Date of submission of the dissertation: $26^{\text {th }}$ September 2014

Date of the oral examination: $1^{\text {st }}$ December 2014 


\section{I hereby confirm that this dissertation}

"In vitro and in vivo characterization of the E3 ubiquitin ligase RNF157 in the brain" was prepared on my own and with no other sources or aids than quoted.

Göttingen, September 2014

Shih-Ju Lee 


\section{Contents}

Acknowledgements............................................................................................................................

Summary .................................................................................................................................................................iii

Abbreviations ................................................................................................................................................iv

1 Introduction .......................................................................................................................................................

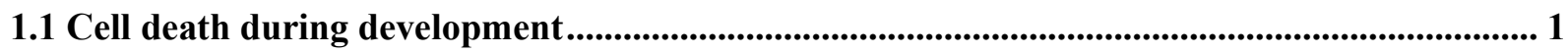

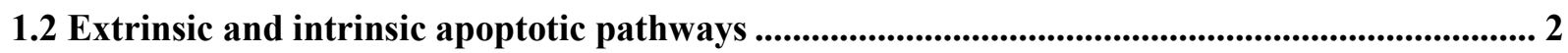

1.2.1 The intrinsic apoptotic pathway is regulated by $\mathrm{Bcl}-2$ proteins ........................................... 5

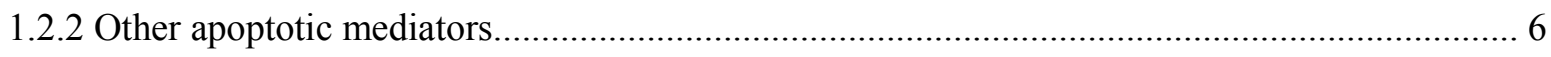

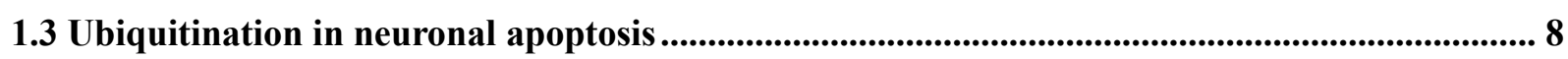

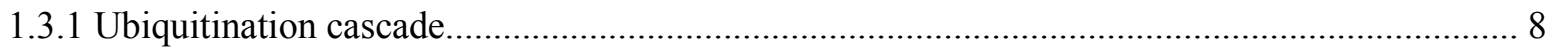

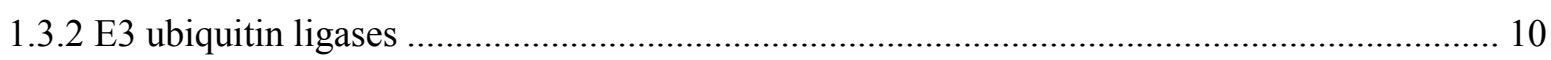

1.3.3 RING-type E3 ligases in apoptosis and neurodegerative diseases ........................................ 12

1.3.4 RING E3 ligases in neurodevelopment and neurodegenerative diseases ............................... 14

1.3.5 RING E3 ligase RNF157 in neuronal apoptosis ............................................................... 18

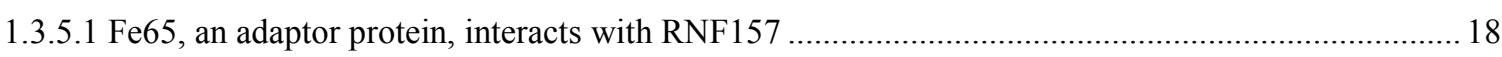

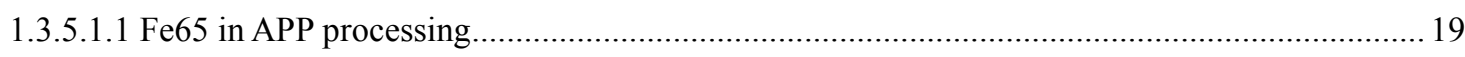

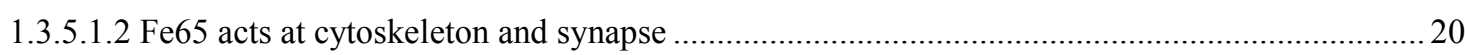

1.3.5.1.3 Fe65 in gene transcription and nuclear signaling ............................................................... 20

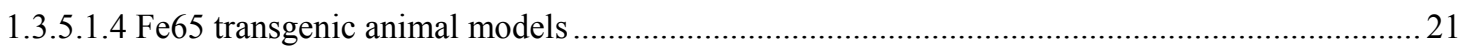

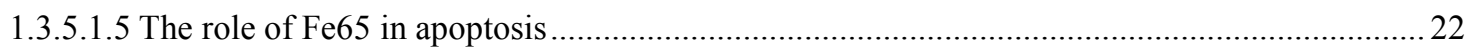

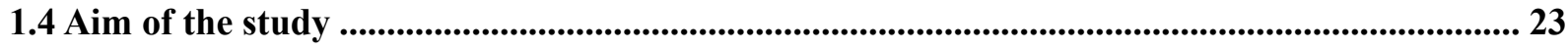

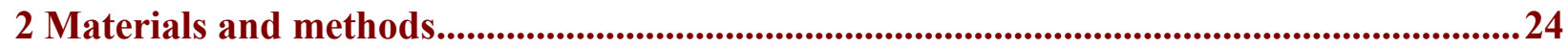

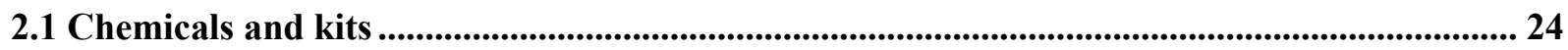

2.2 General Equipment..................................................................................................................... 24

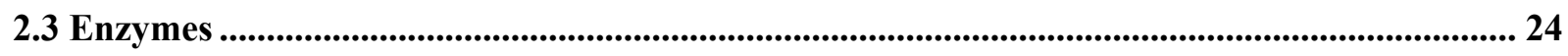

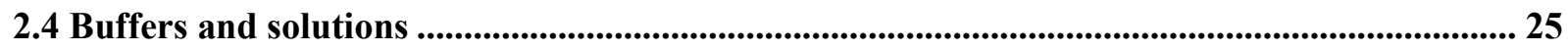

2.5 Expression plasmids and primers................................................................................................. 27

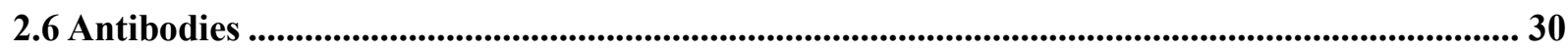

2.7 Expression plasmid construction and cloning ...................................................................................... 32

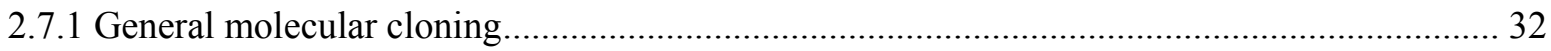

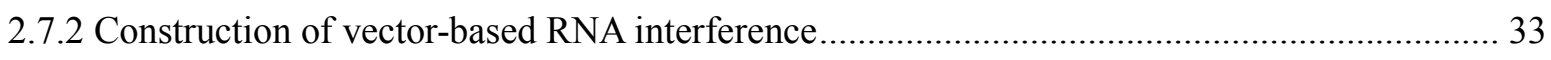

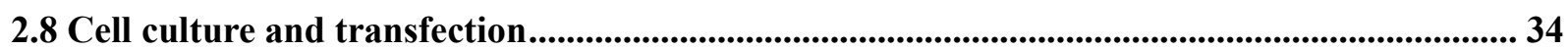

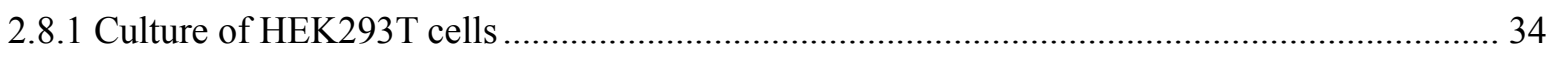

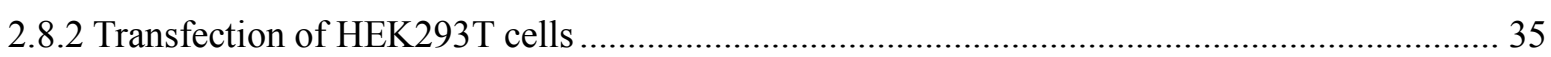

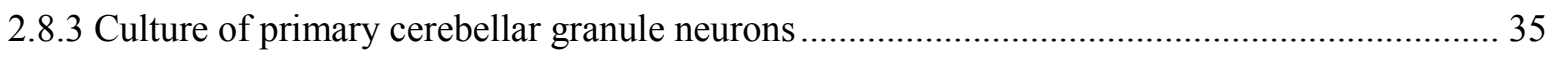

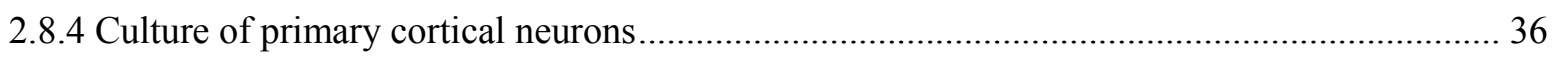




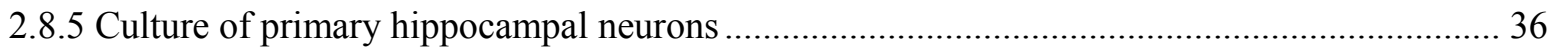

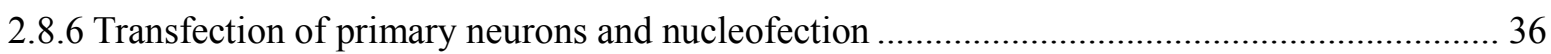

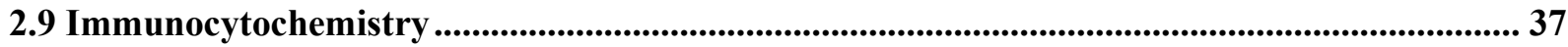

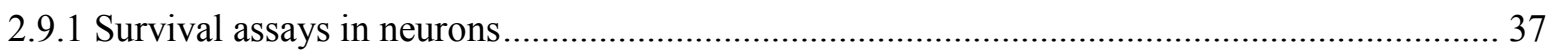

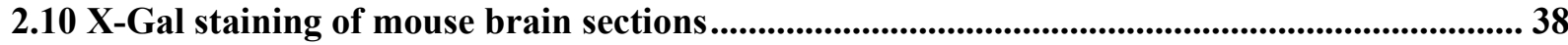

2.11 Biochemical methods................................................................................................... 38

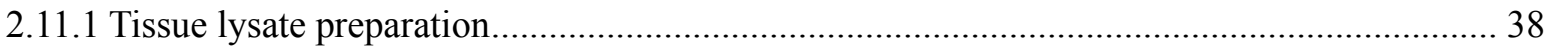

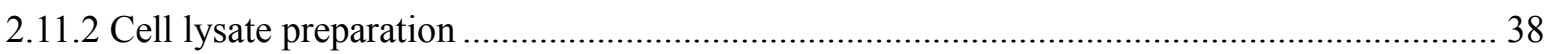

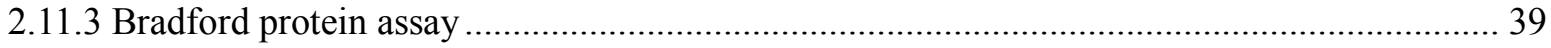

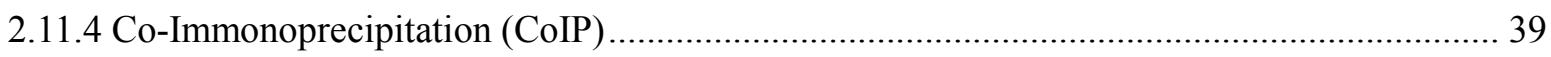

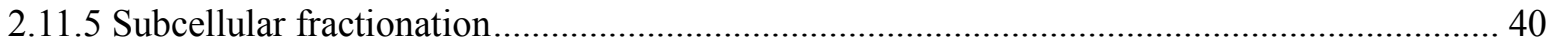

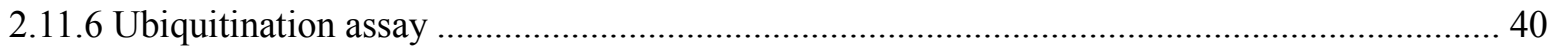

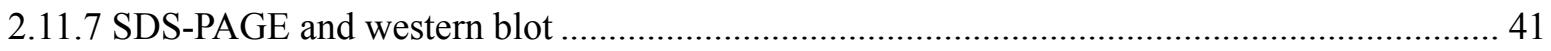

2.11.8 Protein expression and purification ..................................................................... 42

2.12 Generation of RNF157 transgenic mouse ........................................................................ 43

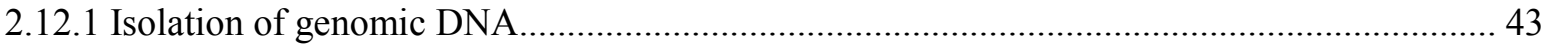

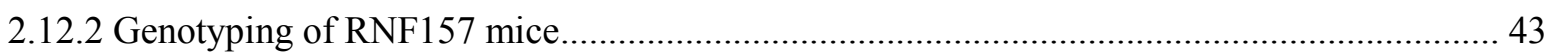

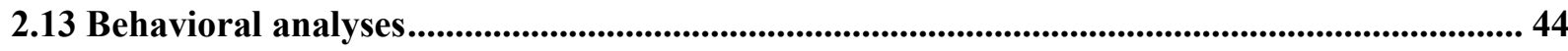

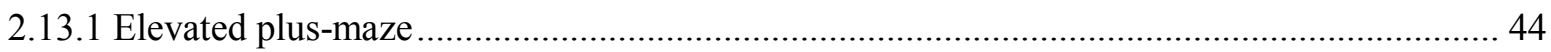

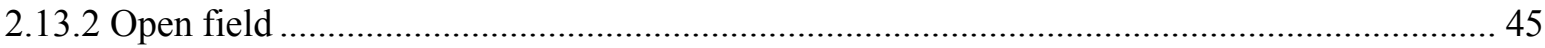

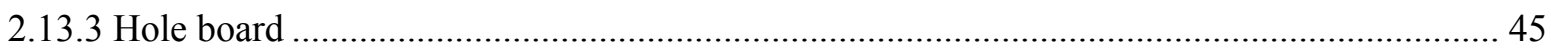

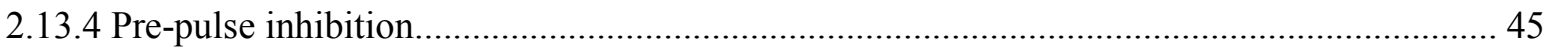

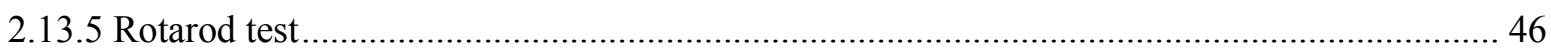

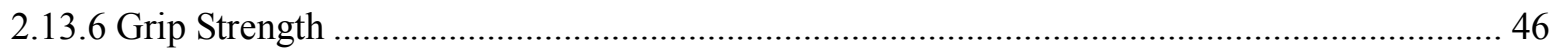

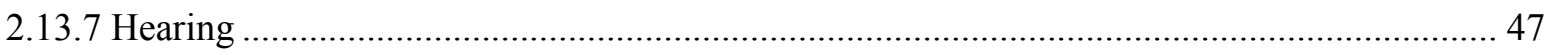

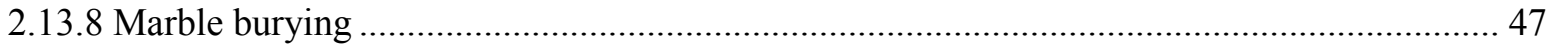

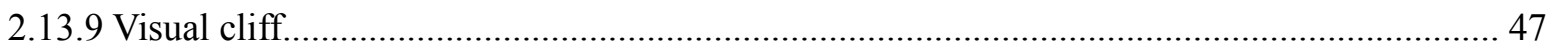

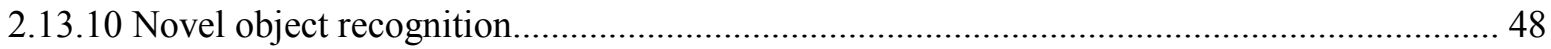

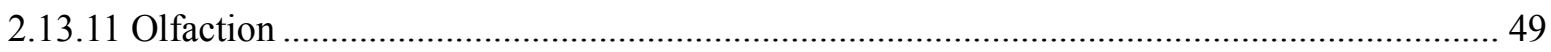

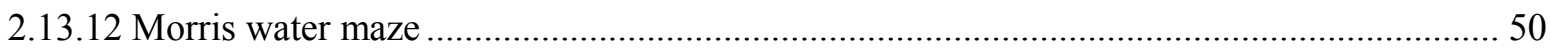

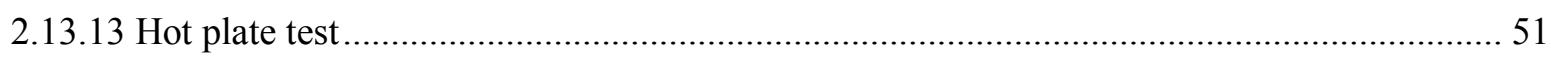

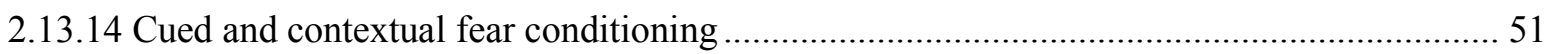

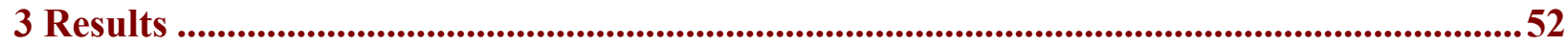

3.1 Characterization of the expression and function of RNF157 ............................................5 52

3.1.1 RNF157 is present in neuronal and non-neuronal cell types in the brain...........................52

3.1.2 RNF157 is strongly expressed in various brain regions .............................................. 53

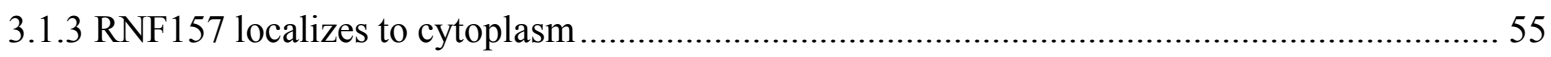

3.1.4 Altered major pathways regulating cell survival due to deletion of RNF157 .....................56

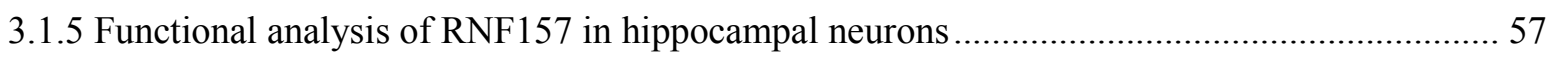

3.2 Fe65, a novel interactor of RNF157, regulates neuronal survival in different neuronal types 59

3.2.1 Fe65 localizes to the nucleus and cytoplasm of cerebellar granule neurons and cortical neurons 
3.2.2 Nuclear Fe65 is the main contributor to cell apoptosis in hippocampal neurons ...................... 60

3.2.3 Fe65 acts downstream of RNF157 to regulate neuronal survival ............................................. 61

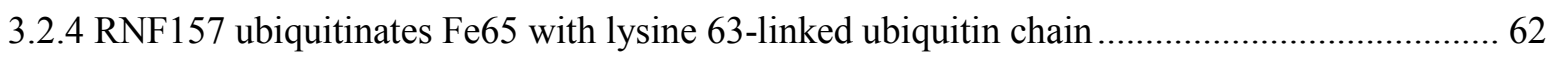

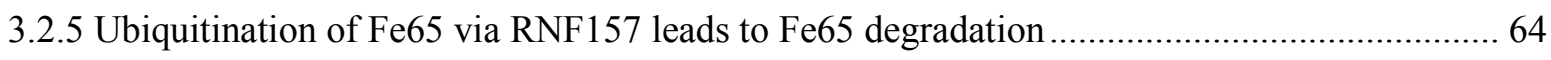

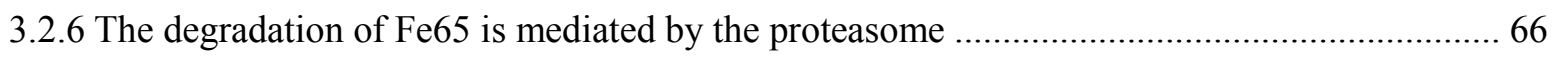

3.3 Potential players involved in the RNF157/Fe65 survival pathway .............................................67

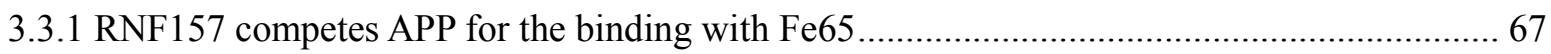

3.3.2 Tip60, interacts with Fe65 at PTB2 domain, does not promote apoptosis .............................. 69

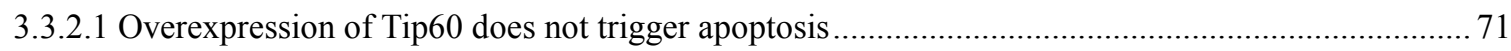

3.3.3 Tip110, a novel interactor of Fe65, localizes to nucleus....................................................... 72

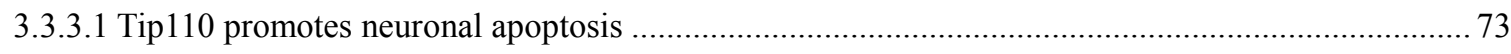

3.3.3.2 Tip110 acts together with Fe65 and RNF157 in controlling neuronal survival ................................ 74

3.4 Behavioral phenotyping of RNF157 knockout mice ...................................................................... 76

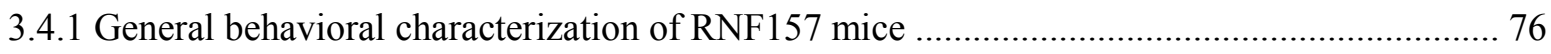

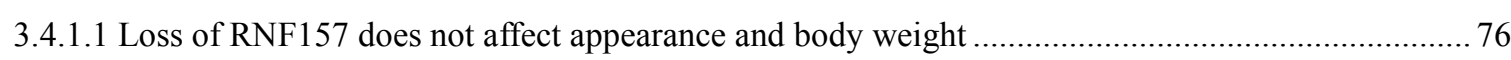

3.4.1.2 Anxiety and locomotor activity is unaltered in RNF157/- mice ................................................... 76

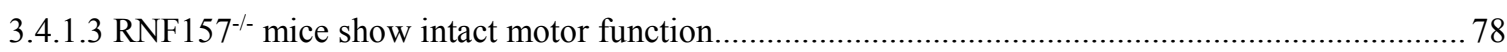

3.4.1.4 Intact sensory and sensorimotor gating functions in RNF157/- mice ........................................... 79

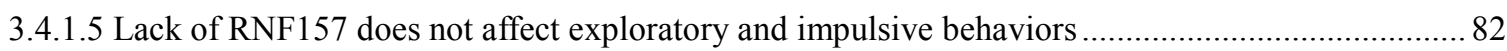

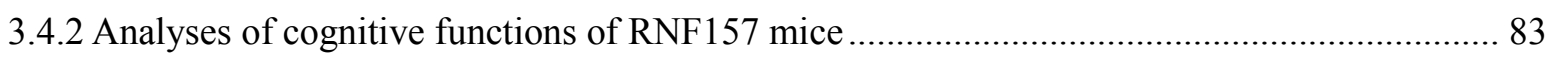

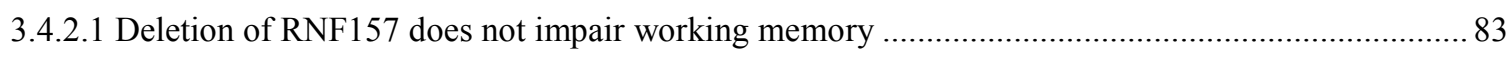

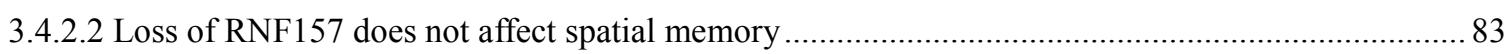

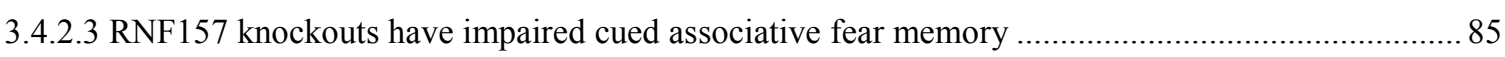

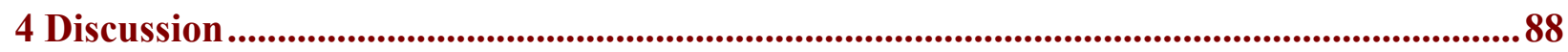

4.1 RNF157 is a brain-dominant protein involved in the Akt- and Erk-mediated survival

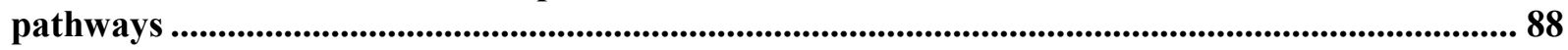

4.2 RNF157/Fe65 pathway regulates apoptosis in hippocampal neurons ........................................ 89

4.3 RNF157 ubiquitinates Fe65 via K63 chains for progressive proteasomal degradation............ 90

4.4 RNF157 competes with APP for the binding of Fe65 ..................................................................... 92

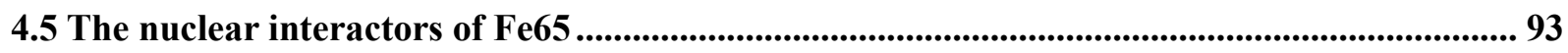

4.5.1 Tip60 interacts with Fe65 but does not promote neuronal apoptosis..................................... 93

4.5.2 Tip110, a novel interactor of Fe65, induces neuronal apoptosis........................................... 96

4.6 The role of RNF157 in cognitive function ..................................................................................... 97

4.6.1 RNF157 has no impact on hippocampus-mediated working and spatial memory .................. 98

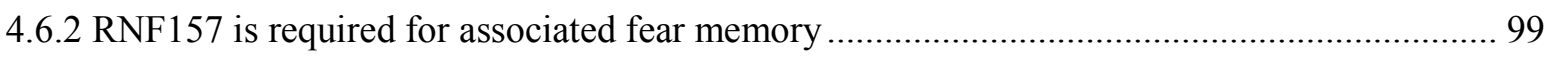

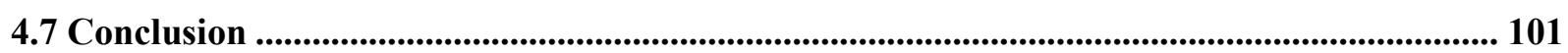

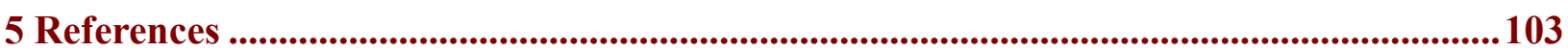




\section{Acknowledgements}

First of all, I would like to thank Dr. Judith Stegmüller for opening the door to molecular neuroscience for me, for guiding me through my $\mathrm{PhD}$ to make my journey as smooth as possible. I deeply appreciate all the encouragement and suggestions for my scientific and future career. Without her, I would not have been where I am today. Her scientific acumen and drive to succeed is truly inspirational.

Furthermore, I would like to thank my thesis committee members Prof. Nils Brose and Prof. Andre Fischer for their time and for the valuable comments and suggestions during every meeting. I would like to additionally thank Prof. Hannelore Ehrenreich, who kindly gave me the chance to complete the behavioral analyses in her group and to attend the fruitful behavioral meetings, which further broadened my scientific view. I also truly appreciate all the great help from the behavioral team.

Next, many thanks go to the most lovely lab mates to ever exist in the world. Firstly, I would like to show my gratitude to Madhu, who accompanied me through the adventure into the unknown scientific world. I appreciate all the knowledge, experiences and emotions that we shared. I thank Mayur for being there whenever I had urgent questions in the lab and also for the lame jokes he made to cheer everyone up. Special thanks go to my project partner Annika, who has always been very supportive through our RNF157 project. Moreover, I would like to thank Nicola for all the great help for my project and my life. I appreciate Anna for giving me all kinds of support that I needed. Big thanks go to Chaitali, who continuously inspired and motivated me through all the brilliant scientific discussions we had. I am really grateful for her proofreading of my English writing and for all the warm emotional support. I thank David for sharing all his professional knowledge with me. I also appreciate David's great contribution to the lab atmosphere by being the Prince of lame jokes. Huge thanks go to Alina, Guergana and Sabitha who are constantly showing the happiness and enthusiasm that reminds me of the beauty of science. Giant thanks go to Siv, who has introduced a lot of different colors into my life. I am deeply grateful for her priceless company and cheering me up at every moment. I greatly appreciate the time that she spent on proofreading of my thesis. I am so glad to be in the same lab with all of you guys!

I would also like to thank all of my friends, who have always been great cheerleaders in my life. In addition, I would like to thank Matthias to share all my ups and downs with me. I am truly grateful for all of his continuous support especially toward the end of my PhD. 
At the end, I would like to express my deepest gratitude to mama, papa, ama, agong, atsim and shushu (媽媽,爸爸,阿嬤,阿公,嬏嬏和叔叔), who have been always on my side for every moment of my life. Without your wise advices and unconditional love, I would never have made it so far. Thank you very much! 
From early neurogenesis to the maintenance of post-mitotic neurons, apoptosis plays a pivotal role. Uncontrolled apoptotic events can lead to abnormal neuronal cell loss, which is a hallmark of various neurodegenerative diseases including Alzheimer's disease, Parkinson's disease or Huntington's disease. As an important protein quality control, the ubiquitin-mediated proteasomal degradation is crucially involved in the apoptotic events. E3 ligases are the most abundant proteins in the ubiquitin-proteasome system that confer the target specificity and catalyze ubiquitination of substrates. Among those, the RING (Really Interesting New Gene)type E3 ligases consisting of more than 600 members represent the most numerous components in ubiquitination.

In this study, I show that the brain-dominant protein RNF157, a RING-type E3 ligase, is present exclusively in the cytoplasm. In addition, together with our previous finding, my functional analyses revealed that RNF157 promotes neuronal survival in hippocampal neurons. Further epistasis analyses demonstrated that the newly identified interactor of RNF157, the adaptor protein Fe65, operates downstream of RNF157 in regulating neuronal survival. Notably, RNF157 ubiquitinates Fe65 via a K63-linked ubiquitin chain and targets Fe65 non-canonically for proteasomal degradation, thus inhibiting the pro-apoptotic function of Fe65.

In addition, I found that nuclear Fe65 is the main contributor in triggering apoptosis in hippocampal neurons as compared to cytoplasmic Fe65. Subsequently, I explored if any nuclear interactors of Fe65 are involved in the RNF157/Fe65 pathway. While the histone acetyltransferase Tip60 does not induce cell death, the novel interactor of Fe65, the RNAbinding protein Tip110, induces neuronal apoptosis. Epistasis analysis indicates that Tip110 acts downstream in the RNF157/Fe65 pathway to regulate neuronal survival.

Furthermore, I investigated if RNF157 has a role in mouse behavior. Using the RNF157 knockout mouse model, I found that loss of RNF157 has no impact on the hippocampusmediated working and spatial memory. Instead, mice lacking RNF157 exhibit impairments in amygdala-mediated fear memory. To sum up, my study discloses a novel RNF157/Fe65/Tip110 pathway in the control of neuronal survival at the cellular level and the importance of RNF157 in cognitive functions. This provides a further insight into the role of E3 ligases in regulating neuronal apoptosis, and thus into mechanisms leading to neurodegeneration. 


\section{Abbreviations}

AA

AD

AICD

AIF

ALS

ANOVA

Apaf1

APC/C complex

APP

$\mathbf{A} \boldsymbol{\beta}$

ATM

ATP

Bcl-2

BDNF

BH

BMP

CAD

CARD

CD95

CDK

CGN

CHIP

CIP

DISC

DIV

DLK

DNA

DR

DTT

amino acid

Alzheimer's disease

amyloid precursor protein intercellular domain

apoptosis-inducing factor

amyotrophic lateral sclerosis

analysis of variance

apoptotic protease-activating factor 1

anaphase-Promoting Complex

amyloid precursor protein

amyloid beta

ataxia-telangiectasia-mutated

adenosine triphosphate

B-cell lymphoma 2

brain-derived neurotrophic factor

Bcl-2 homology

bone morphogenic protein

caspase-activated DNase

caspase activation and recruitment domain

cluster of differentiation 95

cyclin-dependent kinase

cerebellar granule neuron

carboxyl terminus of Hsp70-interacting protein

calf intestinal phosphatase

death-inducing signaling complex

day in vitro

dual leucine zipper-bearing kinase

deoxyribonucleic acid

death receptor

dithiothreitol 


\begin{tabular}{|c|c|}
\hline DUB & deubiquitinating enzyme \\
\hline $\mathbf{E}$ & embryonic day \\
\hline endoG & endonuclease $\mathrm{G}$ \\
\hline EDTA & ethylenediaminetetraacetic acid \\
\hline EGTA & ethyleneglycoltetraacetic acid \\
\hline ERAD & endoplasmic reticulum-associated degradation \\
\hline Erk & extracellular signal-regulated kinase \\
\hline ES & embryonic stem \\
\hline FADD & Fas-associated death domain protein \\
\hline FLIP & FLICE-like inhibitory protein \\
\hline GAN & giant axonal neuropathy \\
\hline GSK3 & glycogen synthase 3 \\
\hline GTPase & guanosine 5'-triphosphate hydrolase \\
\hline HAT & histone acetyltransferase \\
\hline HD & Huntington's disease \\
\hline HECT & homologous to E6AP carboxyl terminus \\
\hline HECTD3 & HECT domain containing E3 ubiquitin protein ligase 3 \\
\hline Het & heterozygous \\
\hline Hom & homozygous \\
\hline HRP & horseradish peroxidase \\
\hline HTN & hippocampal neurons \\
\hline Htt & huntingtin \\
\hline IAP & inhibitor of apoptosis protein \\
\hline ICAD & inhibitor of caspase-activated DNase \\
\hline JNK & c-Jun N-terminal kinase \\
\hline $\mathbf{k b}$ & kilobase \\
\hline kDa & kilodalton \\
\hline LRP & low density lipoprotein receptor-related protein \\
\hline MEK & Ras/Raf/Mitogen-activated protein kinase kinase \\
\hline mg & milligram \\
\hline$\mu g$ & microgram \\
\hline
\end{tabular}




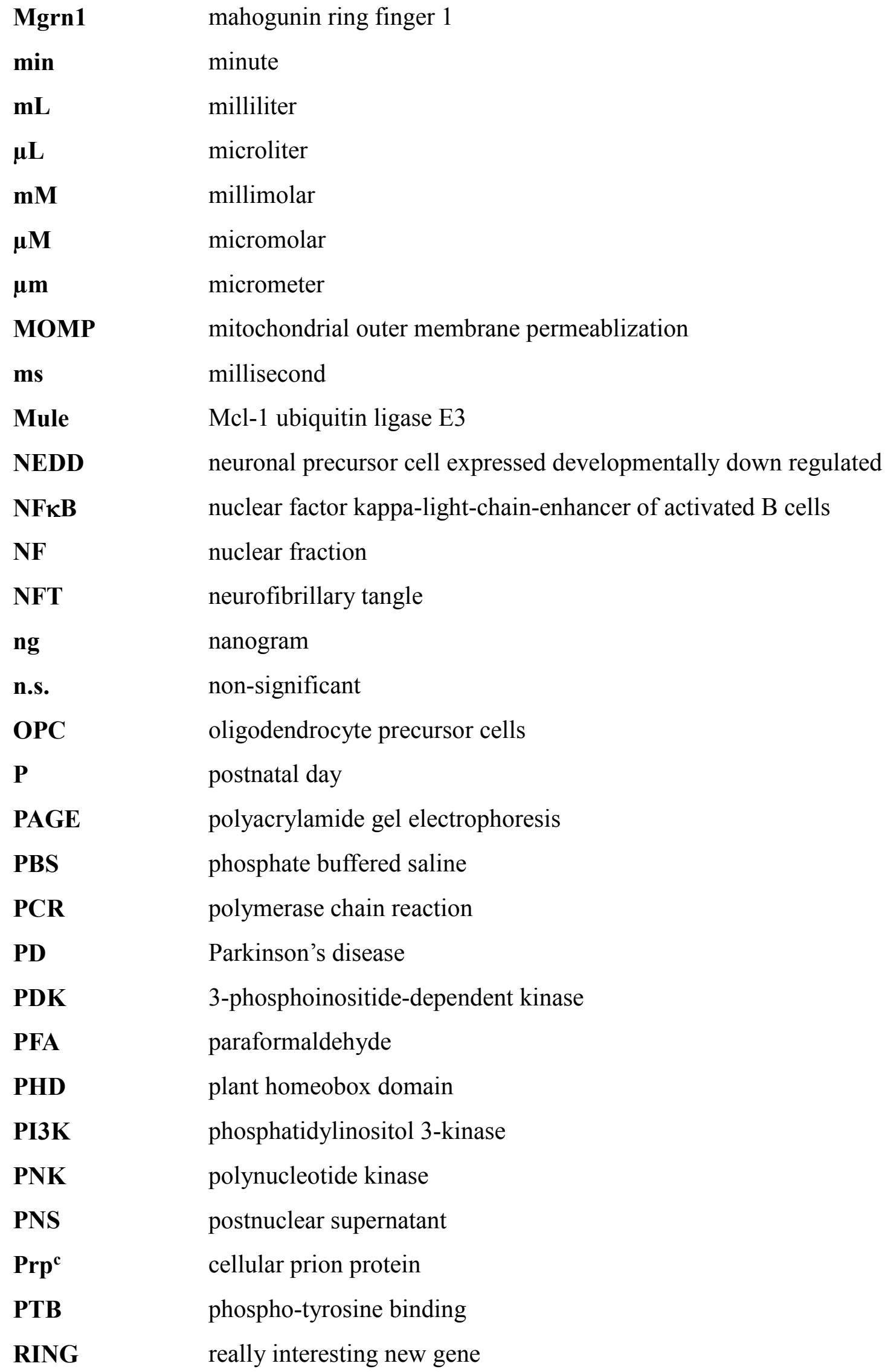


RNA ribonucleic acid

RNAi ribonucleic acid interference

RNF RING finger protein

rpm revolutions per minute

RT room temperature

SCF complex Skp1, Cullin, F-box containing complex

sec second

SDS sodium dodecyl sulfate

s.e.m. standard error of the mean

Siah seven in Absentia homolog

Skp1 S-phase kinase-associated protein 1

Smac Second mitochondria-derived activator of caspase

TEMED tetramethylethylenediamine

Tip HIV-1 tat-interacting protein

TNF tumor necrosis factor

TNFR1 TNF-receptor 1

TRRAP transformation/transcription domain-associated protein

TuJ1 class III beta-tubulin

UPS ubiquitin-proteasome system

VIF vimentin intermediate filaments

WW tryptophan-tryptophan

WT wild type 
Nucleotide

Adenosine

Guanine

Cytosine

Thymine

Amino acid

Alanine

Arginine

Asparagine

Aspartic acid

Cysteine

Glutamic acid

Glutamine

Glycine

Histidine

Isoleucine

Leucine

Lysine

Methionine

Phenylalanine

Proline

Serine

Threonine

Tryptophan

Tyrosine

Valine

Unspecified

\section{Single-letter code}

A

G

C

$\mathrm{T}$

\section{3-letter code}

Ala

Arg

Asn

Asp

Cys

Glu

Gln

Gly

His

Ile

Leu

Lys

Met

Phe

Pro

Ser

Thr

Trp

Tyr

Val
Single-letter code

A

$\mathrm{R}$

$\mathrm{N}$

D

C

E

Q

G

H

I

L

K

M

F

P

S

T

W

Y

V

X 


\section{Introduction}

Our brain works wonders so that we can move and perceive the world, experience and express our emotions, remember and forget things surrounding us. To achieve this, the fascinating network of more than a hundred billion of neurons needs to be precisely controlled. From neurogenesis, neuronal differentiation and migration, synaptogenesis to neuronal maintenance, even the smallest event requires collaborations of numerous molecules. Any mistake can lead to devastating consequences and therefore, we have diverse systems that enable quality control at every step. Even after the brain is fully developed, various triggers can gradually destroy its homeostasis and lead to unwanted loss of neurons. This in turn results in a number of neurodegenerative diseases that can impede our well-being. With this in mind, it is essential to investigate the mechanisms underlying cell death. Only then can we understand how to retain neuronal viability and prevent those precious cells from being destroyed.

\subsection{Cell death during development}

From the embryonic phase throughout the entire life, there is an ongoing controlled loss of cells in every organ of our body. The most studied process mediating this event is apoptosis or programmed cell death, which is evolutionary conserved from plants to animals. In animals, various developmental events are accompanied by apoptosis. One example is the elimination of interdigital webs by genes encoding apoptosis activators or pro-apoptotic proteins (Lindsten et al., 2000; Yoshida et al., 1998). In mice lacking these genes interdigital webs persist and they also develop abnormally enlarged lymphoids (Lindsten et al., 2000). Mutations in apoptosisassociated genes can furthermore lead to the formation of tumors (Lee and Bernstein, 1995; Yip and Reed, 2008). This underscores a fundamental role of the apoptotic machinery in elimination of unnecessary or dangerous cells to ensure early organogenesis and to manage homeostasis of mature tissues.

In addition to its role in non-neural organogenesis, apoptosis plays a critical role in the establishment and refinement of the nervous system. During early development, a proportion of newly born neurons, which were overproduced, undergo apoptosis. The lack of apoptotic signals in knockout mouse models, results in supernumerary neurons, thus leading to malformation of 
the brain (Kuan et al., 2000). Moreover, the excess of post-mitotic neurons is further reduced by the apoptotic process. While deletion of the anti-apoptotic gene Bcl-xL induces massive cell loss among post-mitotic neurons, mice lacking its pro-apoptotic inhibitor Bax retain superfluous proliferating cells (Motoyama et al., 1995; White et al., 1998). In later stages, once neurons have migrated, the access to limited neurotrophic factors is essential for neuronal survival. Neurotrophic factors are released from target cells to attract neurons for synaptic contact. If the neurons fail to capture survival factors, they will undergo apoptosis (Oppenheim, 1991). Taken together, these findings demonstrate the importance of apoptosis throughout neuronal development.

Once the central nervous system is fully formed, mature neurons have very limited capacity to proliferate or to regenerate. Hence, abnormal apoptotic events in this system will disrupt neuronal networks and lead to severe diseases. In this study, I will first elaborate on the apoptotic machinery in neurons and present the key players involved in the regulation of neuronal apoptosis as well as their underlying signaling pathways. Further, I will discuss the consequences of an imbalance of apoptotic pathways in the nervous system.

\subsection{Extrinsic and intrinsic apoptotic pathways}

Various events can trigger apoptosis, including deprivation of neurotrophic factors (Gorin and Johnson, 1980), removal of serum or potassium (Atabay et al., 1996; D'Mello et al., 1993), as well as DNA damage induced by irradiation or other environmental influence (Roos and Kaina, 2013). When a cell undergoes apoptosis, a series of morphological changes can be initially observed: cell shrinkage, chromatin condensation (pyknosis), plasma membrane blebbing and nuclear fragmentation (karyorrhexis). These are followed by the formation of apoptotic bodies containing cell fragments and eventually ingestion and digestion by phagocytes, or, in the brain, by microglia (Elmore, 2007; Erwig and Henson, 2008; Witting et al., 2000).

Two apoptotic pathways manage mainly the incoming apoptotic signals (Fig 1.1). The first is the extrinsic pathway, where extracellular ligands bind to and activate their corresponding death receptors. Death receptors are normally homotrimers and belong to the superfamily of the tumor necrosis factor (TNF) receptors, including CD95 (also known as FAS or Apo1), TNF receptor 1 (TNFR1), death receptor 3 (DR3, also called Apo3 or WSL-1), DR4 and DR5 (also known as Apo2, TRAIL-R2 or KILLER). Members of this family harbor an extracellular ligandbinding domain, a transmembrane domain and an intracellular death domain, which is required 
to activate the apoptosis-signaling cascade. The ligands that bind to these receptors, belong to the TNF superfamily. For example, the CD95 ligand (CD95L) binds to CD95, TNF binds to TNFR1 and Apo3 ligand (Apo3L) binds to DR3. Upon ligand-binding, the cytosolic death domain of the receptor recruits its specific intracellular adaptor protein, such as Fas-associated death domain protein (FADD), which in turn connects to the initiators pro-caspase- 8 or pro-caspase-10. This process forms a death-inducing signaling complex (DISC). If the initiator caspases are not present, the binding sites can be occupied by a cellular caspase-8 (FLICE)-like inhibitory protein (FLIP), which inhibits the induction of apoptotic signals. Once DISC is formed, it activates initiator pro-caspases, which further activate their downstream executioner caspase-3 or caspase7 to trigger apoptosis. (Ashkenazi and Dixit, 1998; Portt et al., 2011; Siegel, 2006; Vucic et al., 2011).

Apoptosis can also be induced by a signaling cascade inside the cell, namely the intrinsic apoptotic pathway. Release of cytochrome c from mitochondria into the cytosol initiates this pathway as a response to the cellular stress such as irradiation or growth factor deprivation. Cytochrome c binds to the apoptotic protease-activating factor 1 (Apaf1), causing the oligomerization of Apaf1 into a wheel-like heptamer called apoptosome. Apaf1 harbors a caspase activation and recruitment domain (CARD) that recruits and activates the pro-caspase- 9 in the apoptosome (Shiozaki et al., 2002; Zou et al., 1997; Zou et al., 1999). The active caspase-9 then activates its downstream effector caspases, caspase-3, caspase-6 and caspase-7, to induce apoptosis (Li et al., 1997; Slee et al., 1999; Srinivasula et al., 1998; Zou et al., 1999).

Furthermore, a cross-talk between extrinsic and intrinsic pathways exists. Depending on the amount of DISC assembly, extrinsic apoptosis proceeds with two different scenarios. In type I cells, sufficient amount of DISC formation leads to direct activation of effector caspases and cleavage of the cell, while in type II cells, lower amount of DISC leads to less activated caspase8 (Scaffidi et al., 1998). Therefore, the intrinsic pathway is required to amplify the apoptotic signals and to execute cell death. Here, caspase- 8 first cleaves Bid, a pro-apoptotic protein belonging to the Bcl-2 family, resulting in the translocation of the truncated Bid (tBid) from cytosol to the mitochondria. This in turn triggers the release of cytochrome c and subsequently activates the intrinsic apoptotic pathway (Li et al., 1998; Luo et al., 1998). 


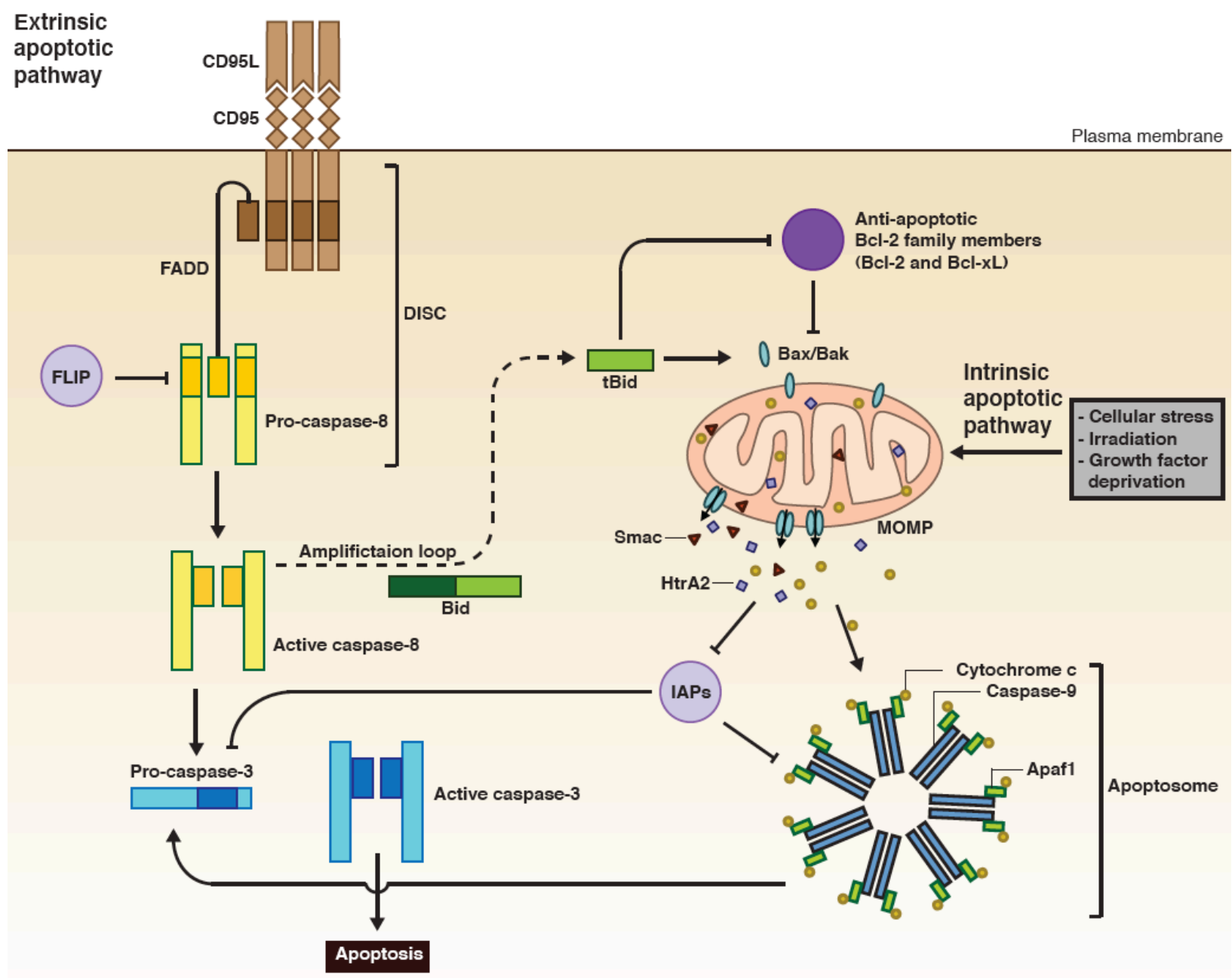

\section{Fig 1.1 Extrinsic and intrinsic apoptotic pathways}

In the extrinsic pathway, a death receptor (here CD95) binds to its ligand (CD95L) to initiate the formation of the death-inducing complex (DISC). This leads to the activation of pro-caspase-8, which directly activates the executioner caspase- 3 and triggers apoptosis. When the amount of active caspase- 8 is not sufficient, the caspase- 8 alternatively cleaves Bid. The truncated Bid (tBid) in turn activates the intrinsic apoptotic pathway. Other intrinsic stimuli including DNA damage, cellular stress or deprivation of growth factors can induce the intrinsic mitochondria-mediated mechanisms. Upon apoptotic signals, the anti-apoptotic Bcl-2 family proteins are inactivated by the pro-apoptotic family members. Meanwhile, Bax or Bak is activated, triggering mitochondrial outer membrane permeablization (MOMP) and the resulting release of mitochondrial proteins such as cytochrome c, Smac and HtrA2. Cytochrome $\mathrm{c}$ then binds to Apaf1, which further recruits caspase-9 to from a complex called apoptosome that facilitates the activation of caspase-9. This is followed by the activation of caspase-3, leading to apoptosis. FLIP, cellular caspase-8 (FLICE)-like inhibitory protein; FADD, Fas-associated death domain protein; IAPs, inhibitors of apoptosis. Modified from Siegel, 2006. 


\subsubsection{The intrinsic apoptotic pathway is regulated by Bcl-2 proteins}

The release of cytochrome $\mathrm{c}$ is the consequence of an event called mitochondrial outer membrane permeablization (MOMP), which is tightly regulated by the family of Bcl-2 proteins. In terms of function, Bcl-2 family members can be divided into either anti-apoptotic or proapoptotic proteins. Besides controlling the exit of cytochrome c from the mitochondria, these proteins can bind to each other to counteract each other's function. In terms of structure, Bcl-2 proteins possess one or more Bcl-2 Homology domains (BH-1, -2, -3 and -4). Anti-apoptotic Bcl-2 members (Bcl-2, Bcl-xL, Bcl-w, A1 and Mcl-1) as well as some of the pro-apoptotic ones (Bax, Bak and Bok) harbor all four BH domains (Kvansakul et al., 2008), whereas the proapoptotic BH3-only members (Bid, Bim, Bad, Puma and etc.) contain a single BH-3 domain (Shamas-Din et al., 2011).

In a healthy cell, Bak is inactive and bound to the outer mitochondrial membrane while Bax is predominantly localized to the cytosol. When a cell receives an apoptotic stimulus, the proapoptotic Bax and Bak aggregate at the outer mitochondrial membrane and oligomerize to trigger MOMP, thus leading to the efflux of cytochrome $\mathrm{c}$ and other pro-apoptotic mitochondrial proteins from the mitochondria into the cytosol to regulate downstream apoptotic events (Gross et al., 1998; Peixoto et al., 2011; Wei et al., 2001).

At the same time, the anti-apoptotic proteins, such as Bcl-2, Bcl-xL and Mcl-1, attempt to prevent MOMP from taking place. These proteins normally localize to intracellular membrane structures such as mitochondria or endoplasmic reticulum (Akao et al., 1994; Hsu et al., 1997; Kaufmann et al., 2003; Perciavalle et al., 2012). They are known to prevent the activation of Bax or Bak and the resulting MOMP by either sequestering Bax or Bak directly or competing with the BH3-only proteins (Billen et al., 2008; Cheng et al., 2001; Willis et al., 2005). In addition, $\mathrm{Bcl}-\mathrm{xL}$ is reported to be involved in the re-translocation of Bax from mitochondria to cytosol (Edlich et al., 2011).

On the other hand, the pro-apoptotic BH3-only members can counteract the anti-apoptotic signals. Unlike the activators Bid, Bim or Puma, which directly activate Bax-Bak to trigger the release of cytochrome c (Chipuk and Green, 2005; Desagher et al., 1999; Kim et al., 2006; Wei et al., 2000), the sensitizer Bad, Bik, Bmf, Hrk or Noxa, sequester the anti-apoptotic Bcl-2 proteins from Bax, Bak or other activators to recover the apoptotic potential (Certo et al., 2006; Kuwana et al., 2005; Letai et al., 2002). In short, the Bcl-2 family plays a key role in regulating apoptosis. 


\subsubsection{Other apoptotic mediators}

After induction of MOMP by the Bcl-2 family, other molecules, apart from cytochrome c, are released from mitochondria to promote or inhibit apoptosis in a caspase-dependent or independent manner. For instance, the second mitochondrial activator of caspases (Smac, also known as Diablo) is a pro-apoptotic protein, which inhibits the activity of the inhibitors of apoptosis (IAP) family. This inhibition leads to the efflux of cytochrome $\mathrm{c}$ and activation of effector caspases (Du et al., 2000; Verhagen et al., 2000). A similar pro-apoptotic molecule is HtrA2 (also called Omi). During apoptosis, mature HtrA2 translocates into cytosol and binds to IAP, thereby eliminating this caspase-inhibition leading to cell death. Besides its caspasedependent modulation, HtrA2 also harbors serine protease activity which induces atypical cell death. This form of HtrA2-induced apoptosis can not be rescued by inhibition of caspases, indicating its role in caspase-independent apoptotic regulation (Hegde et al., 2002; Suzuki et al., 2001a; Verhagen et al., 2002).

Additional mitochondrial proteins are involved in caspase-independent apoptotic pathway: the apoptosis-inducing factor (AIF) translocates to the nucleus during apoptosis, inducing DNA fragmentation and resulting in cell death without caspase-activation (Cregan et al., 2002; Joza et al., 2001; Susin et al., 1999). Another similar mitochondria-localized molecule, the endonuclease $\mathrm{G}$ (EndoG), also moves to the nucleus upon apoptotic signals and triggers caspase-independent DNA degradation (Li et al., 2001; van Loo et al., 2001). In addition, DNA fragmentation can also be mediated by caspases. The non-mitochondrial protein, caspase-activated DNase (CAD), is activated during apoptosis after its inhibitor ICAD is cleaved by caspase-3. The cleavage of ICAD appears to happen in the nucleus upon apoptotic stimuli, leading to the release of active CAD from ICAD. The active CAD then induces DNA fragmentation and chromatin condensation (Durrieu et al., 2000; Enari et al., 1998; Liu et al., 1998; Sakahira et al., 1998).

In addition to these pro-apoptotic mediators, there are further anti-apoptotic regulators, such as ICAD and IAPs. So far, eight IAP members have been identified in humans (Salvesen and Duckett, 2002). IAPs such as XIAP, c-IAP-1 or c-IAP2 promote cell survival by directly binding to the caspases 3, 6 or 7, preventing improper caspase activation and thereby inhibiting apoptosis (Deveraux et al., 1999; Roy et al., 1997).

Other than the abovementioned core regulators in the apoptotic machinery, there are numerous molecules that can affect cell viability. For instance, the neuronal survival pathway mediated by phosphatidylinositide 3-kinases (PI3K)/Akt depends on the activation of PI3k/Akt 
by neurotrophic factors. This in turn activates the pro-survival transcription factor NFKB and results in transcription of survival genes including BcL-xL and IAPs (Brunet et al., 2001). On the other hand, DNA damage can activate the pro-apoptotic gene p53. Activated p53 triggers the transcription of pro-apoptotic genes such as Fas, Bax or Noxa that induces neuronal apoptosis (Nakamura, 2004; Riley et al., 2008).

Besides the transcriptional regulation of apoptotic genes, cell fate is also controlled by various post-transcriptional modifications such as phosphorylation, acetylation, or ubiquitination. Prior to activating its effectors, Akt requires the phosphorylation by PI3K (Hemmings and Restuccia, 2012). Phosphorylated Akt can then further phosphorylate its downstream effectors including the pro-apoptotic protein Bad, thereby inhibiting Bad-induced cell death (Datta et al., 1997). On the contrary, c-Jun N-terminal kinase (JNK) phosphorylates a pro-apoptotic BH-3 member, Bim, upon trophic factor deprivation. This further activates Bax-dependent apoptosis (Putcha et al., 2003). In addition to phosphorylation, the acetylation of p53 by Tip60 is essential for triggering the transcription of Puma, which in turn leads to apoptosis (Tang et al., 2006). Lastly, ubiquitination of various apoptotic regulators can control their stability and activity (Vucic et al., 2011). For example, the anti-apoptotic Mcl-1 can be ubiquitinated by Mule, leading to the elimination of its pro-survival function and thus inducing apoptosis (Zhong et al., 2005). On the other hand, the ubiquitination of caspase- 8 by HECTD3 decreases the caspase- 8 activation, leading to cell survival (Li et al., 2013). These findings underline the importance of protein modifications as a control mechanism of apoptosis so that cell homeostasis can be maintained. 


\subsection{Ubiquitination in neuronal apoptosis}

Ubiquitination is involved in various apoptotic events in different cell types. Among those, neuronal apoptosis, due to disturbed ubiquitination, has drawn increasing attention in the last decades (Ciechanover and Brundin, 2003; Vucic et al., 2011). In the following paragraph, I will address the mechanism of ubiquitination and its implication in regulating apoptosis.

\subsubsection{Ubiquitination cascade}

Ubiquitination is characterized by the process where a 76-amino-acid protein, ubiquitin, is attached to the substrate. An enzymatic cascade requires an E1 ubiquitin-activating enzyme, an E2 ubiquitin-conjugating enzyme and an E3 ubiquitin ligase to catalyze the covalent attachment of ubiquitin onto substrates (Fig 1.2). First of all, the E1 enzyme activates ubiquitin in an ATPdependent manner, leading to the formation of a thioester bond between ubiquitin and its active cysteine residue. The activated ubiquitin is then transferred to an active cysteine residue of an E2 enzyme. The E2-ubiquitin conjugate is further recognized by an E3 ligase that binds target substrates. There are mainly two types of E3 ligases: the HECT-type and the RING-type E3 ligases. The HECT-type E3 ligases harbor a homologous to E6AP carboxyl terminus (HECT) domain containing a catalytic cysteine that recruits the ubiquitin directly from an E2 and forms an E3-ubiquitin thioester intermediate, followed by the transfer of the ubiquitin from the active cysteine to one or more lysine residues of the substrate associated with the E3. On the other hand, members of the really interesting new gene (RING) E3 family act as scaffolds to bring the E2 and substrates in close proximity and thus mediate the transfer of ubiquitin from E2 to the substrates (Berndsen and Wolberger, 2014; Rotin and Kumar, 2009). While E1, E2 and E3 enzymes catalyze the ubiquitination, deubiquitinating enzymes (DUBs) can remove the ubiquitin from the substrate. The cellular signaling mediated by ubiquitination is therefore dependent on the interplay between ubiquitinating versus deubiquitinating enzymes (Reyes-Turcu et al., 2009).

Once a target substrate is ubiquitinated, the type of ubiquitin chains decides its fate. A substrate can be monoubiquitinated, multi-monoubiquitinated or polyubiquitinated. These events can take place at the lysine residues (Lys) of ubiquitin, including Lys6, Lys11, Lys27, Lys29, Lys33, Lys48 and Lys63. The structure of these chains then determines the physiological consequences of target substrates (Fig 1.2b, c). The best-known example is the polyubiquitin chain linked via Lys48 that targets substrate proteins for proteasomal degradation. The Lys11linked polyubiquitin chain also serves in a similar manner. On the other hand, the linear ubiquitin 
chains formed via Lys63 regulate NFאB activation, DNA repair as well as lysosomal degradation. The monoubiquitination or the multi-monoubiquitination also have an important role in mediating protein interaction, localization and substrate activity. Besides these established functions of the aforementioned ubiquitin chains, the role of Lys6, Lys27, Lys29 or Lys33mediated ubiquitination, however, remains unclear (Komander and Rape, 2012; Ye and Rape, 2009).

a

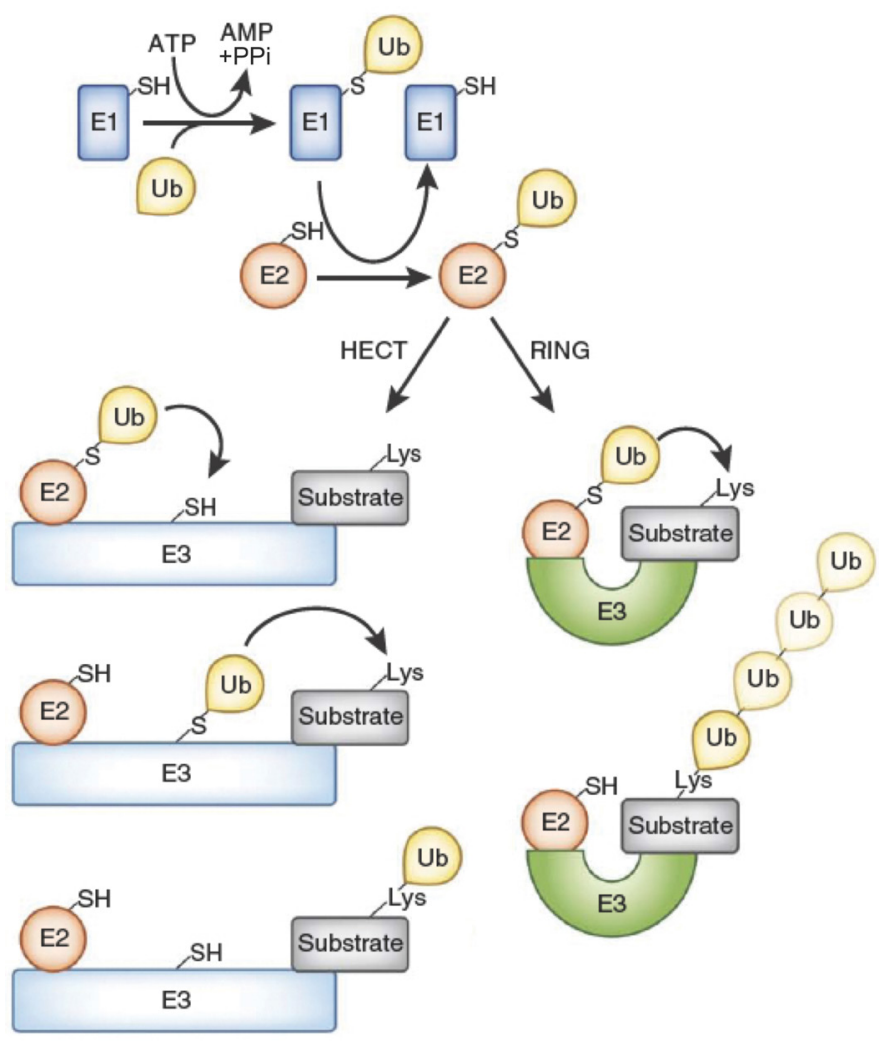

b

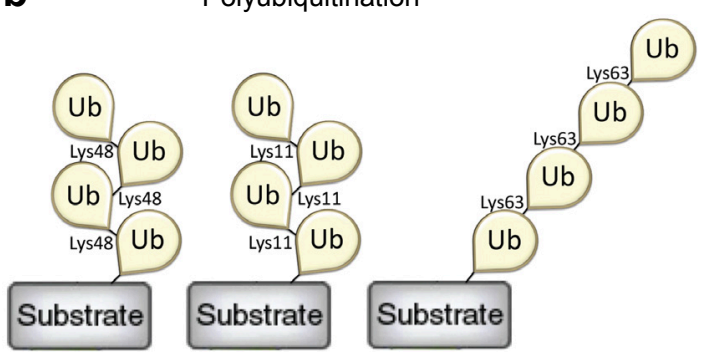

C Monoubiquitination or multi-monoubiquitination

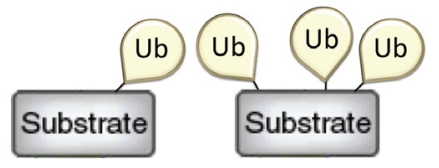

Fig 1.2 Overview of ubiquitination cascade

a) Ubiquitination of target substrates starts with ATP-dependent activation of ubiquitin by an E1 ubiquitinactivating enzyme, leading to the formation of a thioester bond between the E1 and ubiquitin. The activated ubiquitin is then transferred to the active cysteine residue of an E2 ubiquitin-conjugating enzyme. An E3 ubiquitin then facilitates the transfer of ubiquitin onto one or more lysine residues of the substrate protein. One type of E3 ligases harbors a HECT domain (light blue), which recruits ubiquitin from an E2 onto its active cysteine and then transfers the ubiquitin to a lysine of a substrate protein. Another type of E3 ligases possesses a RING domain (green), which recognizes the E2. By bringing the E2-ubiquitin thioester intermediate close to the substrate, a RING E3 facilitates the transfer of ubiquitin from the E2 to the substrate. Modified from Berndsen and Wolberger, 2014.

b) Polyubiquitination via Lys 48 or Lys 11 targets the substrate to the $26 \mathrm{~S}$ proteasome for degradation, while Lys63 linked polyubiquitination regulates NF-kB activation, DNA repair and lysosomal degradation. Modified from Ye and Rape, 2009.

c) Monoubiquitination or multi-monoubiquitination leads to functional modification of substrates, affecting protein interaction, localization or activity. Modified from Ye and Rape, 2009. 


\subsubsection{E3 ubiquitin ligases}

In the ubiquitination system, the substrate specificity is conferred by E3 ligases. This explains why the E3 ligases outnumber the E1 and E2 enzymes. While the human genome encodes two E1 and approx. 30 E2, over 600 E3 enzymes are predicted in a genome-wide analysis (Li et al., 2008b). Among those E3 ligases, approx. 30 are HECT-type while the others are RING-type E3 ligases. HECT-type E3 ligases are characterized by the conserved HECT domain at $\mathrm{C}$-terminus with diverse $\mathrm{N}$-terminal domain structures. These other domains determine substrate targeting (Rotin and Kumar, 2009). In mammals, HECT E3 ligases have been shown to be involved in cell signaling to control various cellular events such as cell growth, proliferation, protein trafficking or DNA damage response. Several HECT E3 ligases also impact on neuronal development as well neuronal survival. For example, Nedd4-1 can mono- or diubiquitinate a small GTPase, Rap2A, to inhibit its activity, and thus promote the function of its effectors leading to dendritic growth (Kawabe et al., 2010). In addition, while Smurfl downregulates a small GTPase, RhoA, to induce axon extension, Smurf2 degrades Rap1B and thus enhances neuronal polarization (Bryan et al., 2005; Schwamborn et al., 2007; Wang et al., 2003). Another HECT E3, E6AP, promotes cell survival in a neuronal cell line and can ubiquitinate p53 for degradation, suggesting that E6AP could protect cells from p53-linked apoptosis (Culmsee and Mattson, 2005; Mishra and Jana, 2008).

With more than 600 members, RING-type E3 ligases represent the largest group of E3 ligases. Unlike HECT-type E3 ligases forming a thioester intermediate with ubiquitin, RINGtype E3 ligases mediate ubiquitin transfer between the E2 and the substrate. RING-type E3 ligases consist of a RING finger and often other functional domains. The canonical RING domain is characterized by a defined spacing of cysteine residues and a histidine (Cys- $\mathrm{X}_{2}$-Xys$\mathrm{X}_{9-39}$-Cys- $\mathrm{X}_{1-3}-\mathrm{His}_{2} \mathrm{X}_{2-3}$-Cys- $\mathrm{X}_{2}-\mathrm{Cys}-\mathrm{X}_{4-48}-\mathrm{Cys}-\mathrm{X}_{2}$-Cys, $\mathrm{X}$ can be occupied by any amino acids), resulting in a "cross-brace" arrangement stabilized by the binding of two zinc ions to the cysteine and histidine residues (Fig 1.3a). In addition, several RING finger variants have been identified; either cysteines or histidines change positions, or one of them is exchanged with other zinc coordinating residues, such as aspartic acid. Not all of the variants possess E3 ligase activity. For instance, LIM and PHD domains share similar organization of cysteins and histidines, but they are not implicated in ubiquitination due to their different folding structures. On the other hand, a derivative of the RING finger, U-Box, is structurally similar to the RING finger and can mediate ubiquitination although the metal-chelating residues of its RING finger are lacking (Aravind and Koonin, 2000). 
a

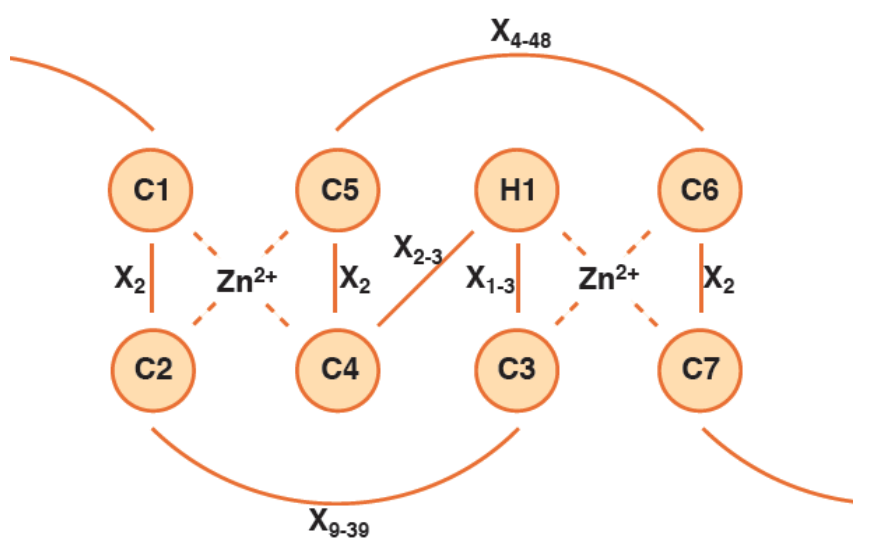

b

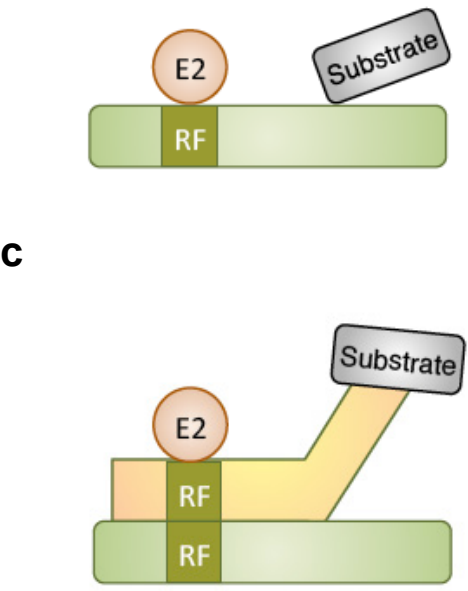

d

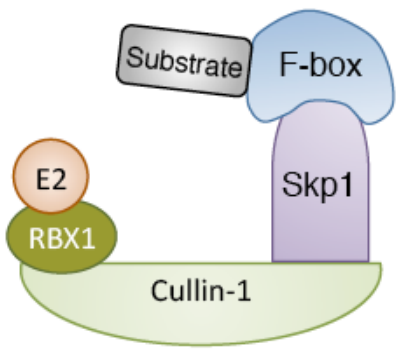

Fig 1.3 The RING E3 ligase and its RING finger motif.

a) The RING finger domain is organized by cysteine and histidine residues with two zinc ions stabilizing its structure. $\mathrm{C} 1$ refers to the first cysteine coordinates with zinc and so on. H1 is the position where histidine is localized. Modified from Deshaies and Joazeiro, 2009.

b-d) RING E3 ligases can act as a monomer (b), dimer (c) or as a multi-subunit complex (d). RING finger domains in RING E3 ligases can interact with E2 and bring E2 closer to the substrate. When RING E3 executes its function in the form of a dimer, only one of the RING domains recruits E2. The binding of the other RING E3 is to enhance

or initiate the E3 ligase activity. The multi-subunit is composed of a scaffold protein, a small RING finger protein, an adaptor protein as well as a F-box subunit for substrate targeting. There are two main groups of RING multisubunit complexes: the SCF and the APC/C E3. In (d), the arrangement of an SCF complex is depicted. Here, Cullin-1 serves as the adaptor protein and RBX1 is the RING-containing protein, which binds to E2. An adaptor protein is Skp1, which interacts with an F-box protein conferring the substrate specificity. Modified from Lipkowitz and Weissman, 2011.

While HECT-type E3 ligases form monomers only, RING-type E3 ligases can act as monomers, dimers or as a multi-subunit complex (Fig 1.3b, c, d, respectively). The dimerization is achieved by binding of two identical RING-type E3 ligases at the RING domains or between a RING E3 and its interactor. Mostly, only one of the RING domains of these dimers acts as the functional recruiter of an E2. The homodimerization of the RNF4 E3 ligase via the RING domain is essential for its activity (Liew et al., 2010). The most complex RING-type E3 ligases are multi-subunit E3 ligases. A multimeric E3 ligase consists of a small RING finger protein, a scaffold protein, several adaptor proteins and a substrate recognition subunit. Among these complexes, the Skp1, Cullin, F-box containing complex (SCF complex) and the anaphasepromoting complex (APC/C complex) have been investigated the most. The SCF complex 
consists of a RING domain containing protein Rbx1, the scaffold protein Cullin-1, the adaptor protein Skp1 and diverse F-box domain proteins, which confer the substrate specificity. The APC/C E3 ligase is more complex and formed by the RING finger protein APC11, the cullinrelated scaffold protein APC2, and a co-activator Cdc20 or Cdh1 as well as at least 11 other subunits. The function of co-activators of the APC/C complex resembles the F-box proteins of the SCF complex, namely to control substrate recognition. In addition, other post-transcriptional modifications, such as phosphorylation or sumoylation, further regulate substrate targeting as well as the activity of RING-type E3 ligases (Deshaies and Joazeiro, 2009; Lipkowitz and Weissman, 2011; Nakayama and Nakayama, 2006). Due to their abundance, RING E3 ligases are involved in almost every physiological event, from regulating cell cycle and maintaining genomic integrity to signaling transduction. Therefore, imbalance of ubiquitination causes various diseases. In neurons, RING-type E3 ligases have also shown their capacity in modulating neuronal migration, neurite outgrowth as well as the neuron viability (Kawabe and Brose, 2011; Weissman et al., 2011). Collectively, E3 ligases are essential to achieve proper cell development and integrity.

\subsubsection{RING-type E3 ligases in apoptosis and neurodegerative diseases}

RING-type E3 ligases are directly involved in major apoptotic pathways. Several members of the anti-apoptotic IAP family as mentioned in chapter 1.1.2 harbor a RING domain in addition to the BIR domains. Take XIAP for example, while the binding of caspase-3 and -7 to BIR1-2 domains of XIAP can already inhibit the activation of caspases, the ubiquitination of mature caspase-9, active caspase-3 as well as Smac mediated by XIAP targets these substrates for degradation and therefore inhibits apoptosis (Deveraux et al., 1999; Morizane et al., 2005; Suzuki et al., 2001b). Other IAPs, such as c-IAP1 and c-IAP2, are also reported to mediate the ubiquitination of Smac, whose subsequent degradation leads to cell survival ( $\mathrm{Hu}$ and Yang, 2003).

Furthermore, upon apoptotic stimuli, IAPs display autoubiquitination activity. Truncated cIAP1, harboring a mutation in the RING domain, is more resistant to autoubiquitination as compared to wild type c-IAP1. The XIAP without RING domain is also less ubiquitinated and thus acts in a protective manner in cells. Since autoubiquitination probably also lowers the cellular apoptotic threshold during apoptosis (Yang et al., 2000), IAPs play an important role in fine-tuning the apoptotic pathway. 
In addition to the direct role of IAPs in apoptosis, numerous RING E3 ligases mediate neuronal viability by providing a suitable cellular environment for the survival of post-mitotic neurons. For example, besides its role in mitosis, the cell cycle regulator Cdh1-APC ligase has been shown to protect post-mitotic neurons from cell cycle re-entry by degrading cyclin B1 and thus inhibiting apoptosis (Almeida et al., 2005; Kruman et al., 2004). Another multi-subunit RING E3, the Fbw7-SCF ubiquitinates phosphorylated c-Jun and targets it for degradation, which results in the disruption of the JNK-mediated neuronal apoptosis (Nateri et al., 2004). Furthermore, an SCF-like E3 complex composed of Cullin-1, the RING-containing protein parkin, as well as an F-box/WD repeat protein hSel-10 targets cyclin E for ubiquitinationmediated degradation (Staropoli et al., 2003). Deletion of parkin results in accumulation of cyclin $\mathrm{E}$ in post-mitotic cultured neurons. Treatment of parkin deficient cultured neurons with the excitotoxin kainate leads to accumulation of cyclin $\mathrm{E}$ followed by apoptosis. In contrast, overexpression of parkin protects neurons from cell death (Staropoli et al., 2003). This suggests a role of parkin in maintaining neuronal survival.

Aside from multi-subunit E3 ligases, various single-subunit E3 ligases are also involved in regulating neuronal apoptosis. For instance, Phr1 ubiquitinates dual leucine zipper-bearing kinase (DLK) for degradation and promotes cell survival (Huntwork-Rodriguez et al., 2013). This survival phenotype can be, however, altered by JNK-mediated phosphorylation of DLK. The phosphorylation of DLK prevents it from being ubiquitinated and degraded, which then inhibits neuronal viability (Huntwork-Rodriguez et al., 2013). Furthermore, Mdm2 is known to ubiquitinate pro-apoptotic protein $\mathrm{p} 53$, which by itself induces apoptosis in neurons. The ubiquitination of p53 via Mdm2 probably leads to the export of p53 from the nucleus to the cytoplasm, where it is degraded by the proteasome and prevents cell death (Fang et al., 2000; Haupt et al., 1997; Jesenberger and Jentsch, 2002; Jordan et al., 1997; Tao and Levine, 1999).

There are also E3 ligases that can directly mediate the intrinsic apoptotic pathway, such as the Trim17 E3 ligase, which belongs to the TRIM/RBCC protein family (Reymond et al., 2001). It acts upstream of mitochondria to enhance neuronal apoptosis (Lassot et al., 2010). While overexpression of Trim17 triggers Bax-dependent apoptosis, knockdown of Trim17 inhibits cell death in cultured neurons. Moreover, deletion of its RING domain abolishes its apoptotic phenotype, indicating that the ligase activity is essential to trigger cell death (Lassot et al., 2010). A subsequent study demonstrates that the Trim17 ubiquitinates an anti-apoptotic Bcl-2 member, Mcl-2, for degradation (Magiera et al., 2013). Taken together, the E3 ligase Trim17 promotes the turnover of Mcl-2 and in turn initiates the Bax-mediated apoptosis. 


\subsubsection{RING E3 ligases in neurodevelopment and neurodegenerative diseases}

One of the major functions of ubiquitination is to target the substrate for degradation. Ubiquitination-mediated proteasomal protein turnover serves as protein quality control, which protects the cell from misfolded proteins or toxic aggregates. Disrupted ubiquitination in neurons can thus lead to abnormal accumulation of these toxic protein species, which are hallmarks of various neurological disorders.

For instance, giant axonal neuropathy (GAN), an early-onset recessive neurological disease, is attributed to mutations in the GAN gene (Kuhlenbaumer et al., 2002). GAN encodes gigaxonin, which is a potential adaptor protein of a multi-subunit E3 ligase together with cullin-3 (Furukawa et al., 2003). Recently, a study has shown that loss of GAN potentiates irregular aggregates of vimentin intermediate filaments (VIF) in $\mathrm{GAN}^{-/-}$mouse embryonic fibroblasts as well as in the cells from patients carrying GAN mutations. Other intermediate filaments (IF) such as neurofilament proteins have also been found to accumulate in patients (Mahammad et al., 2013). These aggregates in the nervous system are believed to induce progressive muscle weakness, disrupted tendon reflexes and disabled walking. As the disease develops further, the central nervous system is affected and patients suffer from dementia, seizures and require life support including artificial nutrition and mechanical ventilation until they die prematurely at around 30 years of age. Although it is clear that a dysfunctional gigaxonin-containing E3 ligase leads to GAN by inducing abnormal aggregation of IFs (Mahammad et al., 2013), it is not known which apoptotic pathway is involved.

Another example is Alzheimer's disease (AD), which is the most common neurodegenerative disease, representing $50-70 \%$ of all dementia cases. It is characterized by agedependent decline of memory and progressive deterioration of cognitive functions. Histopathological analyses of $\mathrm{AD}$ brains reveal brain atrophy in various cognition-related areas, which is a consequence of extensive loss of neuronal cells as well as synaptic connections. The hallmarks of $\mathrm{AD}$ brains are $\beta$-Amyloid plaques and neurofibrillary tangles (NFTs). Emerging evidence shows the presence of ubiquitin in the plaques as well as NFTs, indicating a potential role of ubiquitination during the development of AD (Atkin and Paulson, 2014; Götz and Ittner, 2008).

$\beta$-Amyloid plaques are mainly composed of $A \beta$, a peptide derived from amyloid precursor protein (APP). The cleavage of APP through $\beta$-secretase and the subsequent $\gamma$-secretase leads to the generation of $A \beta_{40}$ or $A \beta_{42}$. While $A \beta_{40}$ is more common and appears to be more 
neuroprotective, $A \beta_{42}$, the major component of plaques, is more neurotoxic (Götz and Ittner, 2008; Gregory and Halliday, 2005). Both of A $\beta$ species can induce apoptosis. While microinjection of $A \beta_{40}$ into mouse cortex leads to caspase-3-mediated cell loss (Takuma et al., 2004), intrahippocampal $A \beta_{42}$ injection in mice also induces caspase-3-associated neuronal loss and related memory deficits (Brouillette et al., 2012), suggesting a link between $A \beta$ and caspasedependent cell death. The production of $A \beta$ can be modified by E3 ligases, such as HRD1, a ligase for endoplasmic reticulum-associated degradation (ERAD). It is one of several E3 ligases that are involved in different stages of APP processing as well as A $\beta$ formation. A reduced level of HRD1 is found in the cortices of AD patients. Overexpression of HRD1 promotes the ubiquitination and degradation of APP, which in turn reduces A $\beta$ production. Accordingly, suppression of HRD1 potentiates APP accumulation as well as A $\beta$ formation, indicating its role in processing of APP (Kaneko et al., 2010). Additionally, a ubiquitin ligase adaptor protein Fbxo2 also has a potential role in regulating APP through ERAD (Atkin et al., 2014). Overexpression of Fbxo2 in a heterologous cells system reduces the APP level, while mice lacking Fbxo2 show an age-dependent upregulation of APP in the brain, indicating that Fbxo2 could be involved in the ubiquitination of APP, which results in APP degradation (Atkin et al., 2014).

The other pathological hallmark of AD, the NFTs, consists of hyperphosphorylated tau. Tau is a microtubule-associated protein, which could regulate cytoskeleton by the binding with actin or tubulin. It is a phosphoprotein with 84 putative phosphorylation sites. Under normal condition, phosphorylated tau is believed to stabilize microtubules, while in the pathological condition, the hyperphosphorylation of tau detaches from microtubule, thereby facilitating the formation of NFTs (Götz and Ittner, 2008; Morris et al., 2011). Upon caspase-3 activation, tau can be cleaved by active caspase-3. Cleaved tau in turn induces cell death. Although the downstream cascade is not yet elucidated, these studies indicate the toxicity of truncated tau (Chung et al., 2001; IdanFeldman et al., 2012). An U-box containing E3 ligase, CHIP, forms a complex with Hsp70, resulting in ubiquitination of phosphorylated tau. In addition, overexpression of CHIP can rescue tau-induced cell death, indicating the CHIP/Hsp70 complex can ubiquitinate tau to attenuate the cellular toxicity (Shimura et al., 2004).

The second most common neurodegenerative disease is Parkinson's disease (PD), characterized by impaired motor functions including tremor, bradykinesia, rigidity, and postural instability. Patients can also suffer from mood disorder, sleep abnormalities or cognitive decline. The deteriorating motor functions can be attributed to the cell death of dopaminergic neurons of 
the substantia nigra. The cause for the cell loss is yet unclear, but various studies indicate a role of $\alpha$-synuclein in regulating neuronal apoptosis in PD (Yasuda et al., 2013). PD patients also show intraneuronal aggregation so-called Lewy bodies, which are mainly composed of misfolded $\alpha$-synuclein. Additionally, mutations in the SCNA gene are involved in familial PD (Andalib et al., 2014; Yasuda et al., 2013). Due to the importance of $\alpha$-synuclein, numerous RING-type E3 ligases that modulate $\alpha$-synuclein are identified. For example, Siah-1 mediates $\alpha$ synuclein ubiquitination, which promotes the aggregation of $\alpha$-synuclein as well as cell apoptosis (Lee et al., 2008a). Furthermore, besides its role in AD, the U-box E3 ligase CHIP is also found in Lewy bodies of PD patients. Overexpression of CHIP can reduce $\alpha$-synuclein accumulation and enhance $\alpha$-synuclein degradation through either proteasomal or lysosomal protein turnover routes, thus protecting cells from $\alpha$-synuclein triggered cell death (Shin et al., 2005).

Moreover, a dysfunction of the E3 ligase parkin is identified in various cases of autosomal recessive PD (Kitada et al., 1998). Parkin can ubiquitinate the pro-apoptotic Bax (Johnson et al., 2012). Instead of degradation, ubiquitination of Bax prevents its stress-induced translocation to mitochondria, thus inhibiting the downstream apoptotic pathway. The disease-related mutation of parkin renders it unable to keep Bax away from mitochondria, leading to enhanced apoptosis (Johnson et al., 2012).

In addition to aforementioned diseases, RING-type E3 ligases are further involved in the amyotrophic lateral sclerosis (ALS), Huntington's disease (HD) as well as spongiform degenerative diseases (Dantuma and Bott, 2014). Patients with familial ALS carry various mutations in the SOD1 gene. Notably, transgenic mice with mutant SOD1 exhibit similar ubiquitin-positive cytoplasmic protein inclusions as shown in patients, although these aggregates are not necessarily SOD1-positive (Watanabe et al., 2001). Mutant SOD1 contributes to caspase1 and -3-mediated motor neuron apoptosis (Pasinelli et al., 2000). Furthermore, mutant SOD1 associates stronger with anti-apoptotic Bcl-2 as compared to wild type SOD1, and thus leads to potential inhibition of Bcl-2 function (Pasinelli et al., 2004). SOD1-induced apoptosis can be rescued by Hsp70, which forms a complex with the E3 ligase CHIP. CHIP does not ubiquitinate mutant SOD1 directly, although only mutant SOD1 interacts with the CHIP/Hsp70 complex. Instead, CHIP mediates ubiquitination of Hsp70. Ubiquitinated Hsp70 then recruits mutant SOD1 and probably translocates together with SOD1 to the proteasome for degradation (Urushitani et al., 2004). This could in turn attenuate mutant SOD1-mediated apoptosis. 
In HD, intranuclear aggregates in neurons as well as dystrophic neuritis are found in patients. Both of the abnormal features are ubiquitin-positive and truncated huntingtin (Htt)-positive, indicating a possible role of the ubiquitin proteasome system in the progress of HD (Becher et al., 1998; DiFiglia et al., 1997). While mutant Htt in the nucleus triggers apoptosis, wild type Htt acts downstream of Bcl-2 and upstream of caspase-3 to protect neurons from cell death (Rigamonti et al., 2000; Saudou et al., 1998). An E3 ligase, TRAF6, binds to mutant Htt, which induces atypical ubiquitination of mutant Htt via Lys6, Lys27 and Lys29-linked chains (Zucchelli et al., 2011). The functional outcome of this ubiquitination, however remains unclear.

Last but not least, recent studies implicate ubiquitin in the spongiform neurodegenerative diseases (Whatley et al., 2008). The hallmark of spongiform degeneration is widespread neuronal or glial cell loss characterized by numerous vacuoles in the central nervous system. CreutzfeldtJakob disease is the best-known one in human and corresponds to scrapie in sheep and bovine spongiform encephalopathy in cow, which are all characterized by progressive motor disturbances and cognitive dysfunctions. The cause of this disease is considered to be the conformational change of a wild type cellular prion protein $\left(\operatorname{Prp}^{c}\right)$ into a pathological isoform, in scrapie called $\operatorname{Prp}^{\mathrm{Sc}}$. The affected brain tissue reveals ubiquitin-positive staining in and around the spongiform structure as well as surrounding the prion plaques. Moreover, various studies also report dysfunction of the proteasome and the resulting alterations in ubiquitination in prion diseases (Whatley et al., 2008). However, the E3 ligases involved in disease progression are not yet identified.

In addition to prion proteins, spongiform neurodegeneration can be directly triggered by loss of an E3 ligase. Mice carrying a point mutation in the RING E3 ligase mahogunin 1 (Mgrn1) gene, which disrupts the transcription of Mgrn1, exhibit progressive widespread spongiform neurodegeneration in the central nervous system (He et al., 2003). The pathological mechanism underlying Mgrn1-deletion is different from the progress of prion diseases but rather a consequence of mitochondrial dysfunction (Silvius et al., 2013). Moreover, Mgrn1 monoubiquitinates TSG101 to regulate the TSG101-mediated endo-lysosomal trafficking of ubiquitinated cargos, although a direct link to neuronal death is still missing (Jiao et al., 2009). Recently, Mgrn1 was reported to be upregulated with Hsp70 in misfolded inclusion bodies upon cellular stress (Chhangani and Mishra, 2013). Overexpression of Mgrn1 restores viability of stressed cells and can enhance degradation of misfolded proteins, while knockdown of Mgrn1 facilitates the release of cytochrome $\mathrm{c}$ followed by apoptosis in cells exposed to stress. Furthermore, Hsp70 appears to potentiate Mgrn1 in protecting cell integrity (Chhangani and 
Mishra, 2013). Although it remains unclear if Hsp70 is a substrate of Mgrn1, this study further supports the notion that Mgrn1 is necessary for cellular homeostasis (Chhangani and Mishra, 2013).

Interestingly, a homologue of Mgrn1, RNF157 (Mgrn2) was introduced in Jiao's study. Mgrn1 and RNF157 share 69\% homology in sequence with a highly comparable N-terminus containing the RING domain (Jiao et al., 2009). While Mgrn1 is expressed throughout the body from brain to periphery tissues including heart, kidney, liver, lung and spleen, RNF157 appears to be predominantly present in the brain (Expression Atlas, EMBL-EBI). Therefore, we hypothesized that RNF157 could have a similar role as Mgrn1 in mammals. Since the function of RNF157 was elusive, we set out to investigate its role in the central nervous system.

\subsubsection{RING E3 ligase RNF157 in neuronal apoptosis}

In the pioneering study, A. Matz has demonstrated a brain-dominant protein expression of RNF157, which is consistent with the finding that RNF157 mRNA is robustly expressed in the brain, while only to a smaller extent in lung, heart and kidney (Matz et al., in press). In addition, RNF157 protein is stably expressed in cultured cerebellar granule neurons as well as in cortical neurons. Using whole tissue lysate, she also confirmed that throughout brain development from embryonic day (E) 18 to postnatal (P) 60, protein expression of RNF157 is downregulated in the cortex, upregulated in cerebellum and unchanged in hippocampus. Intriguingly, RNF157 was found to promote cell survival in primary cerebellar granule neurons. While knockdown of RNF157 triggers neuronal apoptosis, overexpression of RNF157 protects neurons from cell death induced by serum deprivation (Matz et al., in press). Moreover, the mutant RNF157 lacking the catalytic RING domain fails to rescue neurons from death, suggesting that RNF157 promotes neuronal survival in a ligase-activity-dependent manner (Matz et al., in press).

\subsubsection{Fe65, an adaptor protein, interacts with RNF157}

The story took an interesting turn when an interaction partner of RNF157, Fe65, was identified in our previous yeast two-hybrid screening (Matz et al., in press). Fe65 is a member of the Fe65s family including Fe65L1 and Fe65L2. While Fe65L1 and Fe65L2 are widely expressed, Fe65 reveals a brain-enriched expression pattern (Guenette et al., 1996a; Sabo et al., 2003; Tanahashi and Tabira, 1999). Each member harbors a tryptophan-tryptophan (WW) 
domain and two phospho-tyrosine binding (PTB) domains (Fig 1.4a). The mapping analyses demonstrated that Fe65 associates with N-terminal region of RNF157 excluding the RING domain, and RNF157 binds to the c-terminal PTB2 domain of Fe65 (Fig 1.4b) (Matz et al., in press). Fe65s act as adaptor or scaffold proteins to form protein complexes. Depending on the binding partners of Fe65s, they are involved in various cellular events such as APP trafficking and processing, cell motility, synaptic transmission and nuclear signaling (Borquez and Gonzalez-Billault, 2012; Matz et al., in press; McLoughlin and Miller, 2008)

a

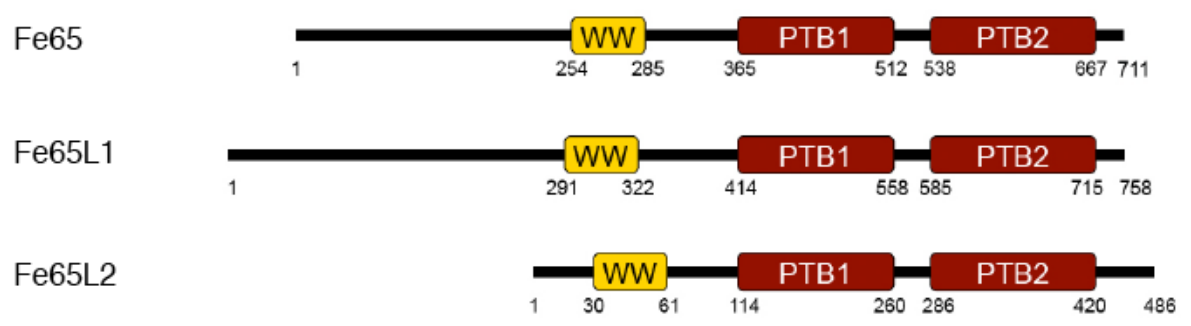

b

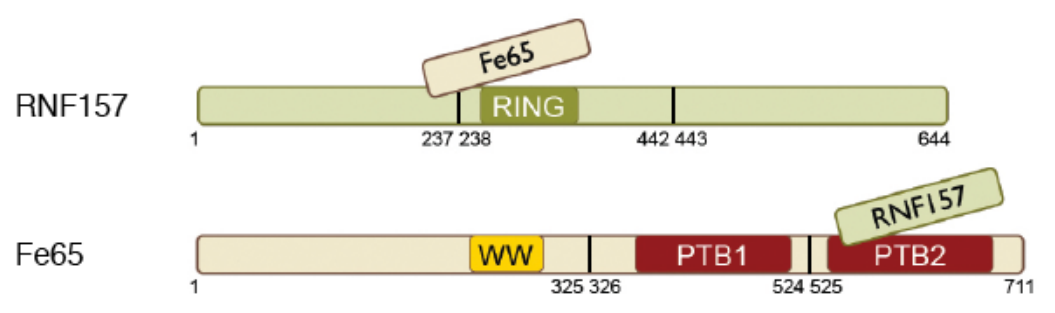

Fig 1.4 Fe65s and its interaction with RNF157.

a) Domain structure of human Fe65s. Modified from McLoughlin and Miller, 2008.

b) Fe65 binds to the N-terminus of RNF157 exclusive of the RING domain, and RNF157 associates with the PTB2 domain of Fe65.

\subsection{Fe65 in APP processing}

Fe65s drew a lot of attention owing to the interaction with APP, which implicates a role in Alzheimer's disease. All Fe65 members of the family can bind to APP (Kimberly et al., 2001; McLoughlin and Miller, 1996; Tanahashi and Tabira, 1999). The interaction between the PTB2 domain of Fe65 and the YENPTY motif in the intracellular domain of APP (AICD) is of particular interest. This binding stabilizes AICD and colocalizes Fe65 and AICD in the nucleus (Kimberly et al., 2001). Furthermore, mutations in the YENPTY motif in APP block its endocytic trafficking and decreases $A \beta$ formation (Perez et al., 1999). Therefore, it is possible that Fe65 binds to APP to mediate the nuclear translocation of AICD and Fe65 thus influence A $\beta$ 
production. Furthermore, while APP binds to the PTB2 domain of Fe65, the low-density lipoprotein receptor-related protein (LRP) can bind to Fe65's PTB1 domain (Trommsdorff et al., 1998). This leads to the formation of a trimeric complex consisting of Fe65, APP and LRP, which is also involved in regulating APP processing (Pietrzik et al., 2004). Overexpression of LRP enhances Fe65-mediated APP processing (Pietrzik et al., 2004), whereas Dab1, another adaptor protein with a PTB domain, can bind to LRP to counteract the impact of Fe65 or LRP on APP cleavage (Kwon et al., 2010). In addition, Dab1 competes with Fe65 for the binding to APP, resulting in the release of Fe65 from APP and thus facilitating the nuclear translocation of Fe65 as well as suppressing the Fe65-mediated APP processing (Kwon et al., 2010). Besides the binding to LRP, Fe65 can also bind to the apoE receptor ApoEr2 and may in turn form a complex with APP to modulate APP processing (Hoe et al., 2006). While ApoEr2 or Fe65 by itself can enhance APP processing, the Fe65/ApoEr2 complex further increases the amount of membranebound c-terminal APP fragment (Hoe et al., 2006; Hoe et al., 2005). These findings support the notion that Fe65, together with its interacting proteins, is essential for the cleavage of APP.

\subsection{Fe65 acts at cytoskeleton and synapse}

Moreover, Fe65 binds to tau via its PTB1 domain. This complex colocalizes with APP in primary neurons, implicating a role of Fe65 in the regulation of the cytoskeleton (Barbato et al., 2005). In addition, Mena, an Abl-associated signaling protein involved in regulating actin dynamics, interacts with Fe65 through its WW domain (Ermekova et al., 1997). It associates with APP and Fe65 simultaneously and colocalizes with actin, Fe65 and APP in lamellipodia (Sabo et al., 2001). Since overexpression of APP and Fe65 accelerates cell migration (Sabo et al., 2001), it is speculated that the APP/Fe65/Mena complex acts at the cytoskeleton to regulate movement. Furthermore, Fe65 is present at synapses and binds to P2X 2 subunits (Masin et al., 2006). $P 2 X_{2}$ is a ligand-gated ion channel activated by extracellular ATP and is involved in synaptic transmission. Overexpression of Fe65 hinders the permeability change of $\mathrm{P}_{2} \mathrm{X}_{2}$ receptors (Masin et al., 2006), suggesting a role of Fe65 in synaptic transmission.

\subsection{Fe65 in gene transcription and nuclear signaling}

In addition to the aforementioned functions, nuclear Fe65 acts with various proteins in the nucleus to regulate gene transcription. Upon the binding to the transcription factor late SV40 
factor (LSF) via its PTB1 domain, Fe65 blocks LSF-mediated transactivation of the thymidylate synthase gene. Interestingly, overexpression of APP counteracts this inhibition (Bruni et al., 2002). Moreover, as already mentioned, Fe65 can translocate into the nucleus upon binding to AICD. Once Fe65 and AICD have entered the nucleus either separately or together, Fe65 binds to the histone acetyltransferase (HAT) Tip60 to initiate the transcription of target genes (Cao and Südhof, 2001; Kimberly et al., 2001). However, the transcription can be suppressed by Dexras1, a Ras family small G protein (Lau et al., 2008). Dexras1 binds to Fe65 at its PTB2 domain. Hence, it competes with APP for the association with Fe65. Once APP-Fe65 binding is disrupted, the APP-Fe65-mediated transcription is subsequently blocked (Lau et al., 2008).

Fe65 is also involved in DNA damage response. When DNA damage occurs, Tip60 forms a complex with TRRAP, a cofactor of HAT complexes, to acetylate histone H4 at DNA double strand breaks and in turn, promote DNA repair (Murr et al., 2006). Upon DNA damage, deletion of Fe65 suppresses the recruitment of Tip60-TRRAP to the damage site and therefore hinders DNA repair. Interestingly, APP is required for Fe65-mediated DNA repair (Stante et al., 2009). Knockdown of APP restricts Fe65-induced recruitment of Tip60-TRRAP and thus decreases DNA repair efficiency. A similar series of studies using sorbitol to induce DNA damage supports this finding (Cao and Südhof, 2001; Nakaya et al., 2008, 2009; Tang et al., 2006). Under osmotic stress induced by sorbitol, Fe65 enters the nucleus mediated by APP and AICD. Nuclear Fe65 in turn prevents p53 from proteasomal degradation, perhaps regulated by the binding between Fe65 and Tip60 as well as the Tip60-induced acetylation of p53. The stabilized p53 then facilitates the phosphorylation of the histone $\mathrm{H} 2 \mathrm{AX}(\gamma \mathrm{H} 2 \mathrm{AX})$ and triggers either DNA repair or apoptosis. Taken together, these findings support that APP-regulated Fe65 translocation into nucleus is required for the nuclear signal transduction in response to DNA damage.

\subsection{Fe65 transgenic animal models}

To study the role of Fe65 in vivo, various transgenic models have been generated. Ablation of feh-1 gene, the only orthologue of Fe65 in Caenorhabditis elegans, leads to arrest during the late embryonic period or at the first larval stage L1, suggesting that Fe65 is required for proper development of C. elegans (Zambrano et al., 2002). The single Fe65 knockout mouse are grossly normal but with subtle impairments. Deletion of Fe65 affects neurogenesis and these mice exhibit reversal learning deficits in Morris water maze at 14 months of age, indicating a role of Fe65 in neurodevelopment and cognition (Ma et al., 2008; Wang et al., 2004). A similar study 
demonstrates that mice lacking either Fe65 or Fe65L1 appear to be apparently normal, while the Fe65/Fe65L1 double knockout mice have severe neurodevelopmental defects including disorganized cortices and hippocampi resulting from aberrant midline crossing and abnormal axonal projections (Guenette et al., 2006). Furthermore, mouse embryonic fibroblasts isolated from $\mathrm{Fe} 65^{-/-}$are more sensitive to irradiation, accelerating rapid upregulation of $\mathrm{p} 53$ and $\gamma \mathrm{H} 2 \mathrm{AX}$ (Minopoli et al., 2007). These findings suggest that Fe65 is crucial for proper neurodevelopment as well as DNA damage response in vivo.

\subsection{The role of Fe65 in apoptosis}

So far, studies concerning the role of Fe65 in cell viability demonstrate that overexpression of Fe65 either does not induce cell death in HEK293T and SH-SY5Y cells, or augments apoptosis in CHO, HEK293T as well as Neuro 2A cells (Chow et al., 2012; Kim et al., 2007b; Lee et al., 2009a; Vazquez et al., 2009). The discrepancy could be attributed to different experimental procedures. On the other hand, knockdown of Fe65 is shown to partially attenuate $\mathrm{H}_{2} \mathrm{O}_{2}$-induced apoptosis in HEK293T cells (Kim et al., 2012). These findings suggest a potential pro-apoptotic function of Fe65. However, the role of Fe65 in neuronal apoptosis was not well investigated.

The study by Matz and colleagues demonstrated that overexpression of Fe65 triggers apoptosis in cerebellar granule neurons, while knockdown of Fe65 prevented neurons from serum-deprivation-induced cell death (Matz et al., in press). Further survival analyses using Fe65 deletion mutants revealed that the PTB2 domain of Fe65 was localized to the nucleus and was most potent in initiating apoptosis. Having established that Fe65 interacted with RNF157 and served as an apoptotic inducer in cerebellar granule neurons, subsequent epistasis analyses were carried out. The result exhibited that Fe65 acted downstream of RNF157 to regulate apoptosis in cerebellar granule neurons (Matz et al., in press). 


\subsection{Aim of the study}

The aim of my study was to expand the understanding of how the RING-type E3 ligase RNF157 and its interactor Fe65 work in the brain. Using the RNF157 knockout mouse model, I first elucidated the spatial distribution of RNF157 in vitro and in vivo. Next, I characterized the function of RNF157 as well as the role of its interactor Fe65 in hippocampal neurons. Furthermore, I sought to identify possible molecules that are involved in the RNF157/Fe65 survival pathway. In addition to the establishment of an RNF157-dependent linear survival pathway in vitro, I aimed at understanding the functional implication of RNF157 in vivo. I therefore conducted a comprehensive behavioral test battery in the RNF157 knockout mice. Utilizing biochemical and immunochemical methods as well as behavioral analysis, my study provides a comprehensive view of the role of RNF157 in neuronal viability and cognition in the central nervous system. 


\section{Materials and methods}

\subsection{Chemicals and kits}

Unless mentioned in the text, chemicals and reagents used in this study were purchased from AppliChem (Darmstadt, Germany), Roth (Karlsruhe, Germany), Merck (Darmstadt, Germany), Becton Dickinson and Company (Heidelberg, Germany), Sigma-Aldrich (Steinheim, Germany) or Invitrogen (Darmstadt, Germany).

Human Fetal Brain Mate \& Plate ${ }^{\circledR}$ Library was from Clontech Laboratories, Inc. (Mountain View, USA). NucleoSpin ${ }^{\circledR}$ Plasmid QuickPure kit, NucleoSpin ${ }^{\circledR}$ Gel and PCR Clean-up kit, and NucleoBond ${ }^{\circledR}$ Xtra Midi EF kit were from Macherey-Nagel (Düren, Germany).

\subsection{General Equipment}

The following equipment was used in the experiments: plastic wares such as $1.5 \mathrm{ml}$ microcentrifuge tubes, pipette tips, reaction tubes $(15$ or $50 \mathrm{ml})$ or culture dishes were from Eppendorf (Hamburg, Germany), Sarstedt AG (Nürnbrecht, Germany), Gilson (Middleton ,USA) or BioOne (Frickenhausen, Germany), centrifuges from Eppendorf, rocker and shaker from Heidolph (Schwabach , Germany), thermocycler from Biometra (Göttingen, Germany), heat block from Grant Instruments (Shepreth, UK), and spectrophotometer from GE Healthcare (Freiburg, Germany). Microscopic images were taken with an SP2 confocal microscope from Leica (Solms, Germany) or with an inverted light microscope (Exlipse TS100) from Nikon (Tokyo, Japan). Dissection microscope (SMZ645) was from Nikon.

\subsection{Enzymes}

Enzymes used in this study are listed below:

\begin{tabular}{l|l} 
Enzyme & Supplier \\
\hline Pfu DNA polymerase & Fermentas
\end{tabular}




\begin{tabular}{l|l} 
Restriction Enzymes & New England Biolabs \\
\hline Alkaline phosphatase, calf intestinal (CIP) & New England Biolabs \\
\hline T4 DNA Ligase & Fermentas \\
\hline T4 DNA Polymerase & Fermentas \\
\hline T4 Polynucleotide Kinase & New England Biolabs \\
GoTaq ${ }^{\circledR}$ DNA polymerase & Fermentas \\
PCR SuperMix & Invitrogen
\end{tabular}

\subsection{Buffers and solutions}

The buffers and solutions listed bellow were frequently used in various experiments:

TX-100 lysis buffer: $150 \mathrm{mM} \mathrm{NaCl,} 50 \mathrm{mM}$ Tris $\mathrm{HCl} \mathrm{pH}$ 7.5, $1 \mathrm{mM}$ EDTA, 1\% Triton X-100

Running buffer: $25 \mathrm{mM}$ Tris, $250 \mathrm{mM}$ glycine, $0.1 \%$ SDS

Transfer buffer: $190 \mathrm{mM}$ glycine, $24 \mathrm{mM}$ Tris, $0.0375 \%$ SDS, 20\% methanol

Lower buffer: $1.5 \mathrm{M}$ Tris $\mathrm{pH} 8.8,0.4 \%$ SDS

Upper buffer: $0.5 \mathrm{M}$ Tris $\mathrm{pH} 6.8,0.4 \%$ SDS

4x SDS sample buffer: $25 \mathrm{ml}$ Upper Buffer, $20 \mathrm{ml}$ Glycerol, 4 g SDS, $2 \mathrm{ml}$ 2-Mercaptoethanol, $2 \mathrm{~g}$ Bromphenolblue (stored in $1 \mathrm{ml}$ aliquots at $-20^{\circ} \mathrm{C}$ )

PBS: $136 \mathrm{mM} \mathrm{NaCl}, 2.68 \mathrm{mM} \mathrm{KCl}, 4.29 \mathrm{mM} \mathrm{Na}_{2} \mathrm{HPO}_{4}$ x 7H $2 \mathrm{O}, 1.47 \mathrm{mM} \mathrm{KH}_{2} \mathrm{PO}_{4}$

PBST: PBS, $0.1 \%$ Tween 20

2xYT media (1L): $16 \mathrm{~g}$ tryptone, $10 \mathrm{~g}$ yeast extract, $5 \mathrm{~g} \mathrm{NaCl}$

Annealing buffer: $100 \mathrm{mM}$ potassium acetate, $30 \mathrm{mM}$ Hepes, $2 \mathrm{mM}$ magnesium acetate

8\% PFA: $375 \mathrm{ml}$ sterile $\mathrm{H}_{2} \mathrm{O}$ at $60^{\circ} \mathrm{C}, 40 \mathrm{~g}$ of PFA, $50 \mathrm{ml} \mathrm{10x}$ PBS and $40 \mathrm{~g}$ sucrose; fill up with sterile $\mathrm{H}_{2} \mathrm{O}$ to final volume of $500 \mathrm{ml}, \mathrm{pH} 7.4$ 
Co-IP buffer: $150 \mathrm{mM} \mathrm{NaCl}, 20 \mathrm{mM}$ Tris $\mathrm{pH}$ 7.4, $1 \mathrm{mM}$ EDTA, 1\% Nonidet P-40, 10\% Glycerol

RIPA buffer: $50 \mathrm{mM}$ Tris-HCl $\mathrm{pH} 8.0,150 \mathrm{mM} \mathrm{NaCl}, 1 \% \mathrm{NP} 40,0.5 \%$ Sodium deoxycholate, $0.1 \%$ SDS, $5 \mathrm{mM}$ EDTA

Buffer A: 10 mM HEPES pH 7.9, 10 mM KCl, 0.1 mM EGTA, 0.1 mM EDTA

Buffer $\mathrm{A}^{+}$: Buffer A, 0.1\% Nonidet P-40

Buffer C: 20 mM Hepes pH 7.9, 400 mM NaCl, 1 mM EDTA, 1 mM EGTA

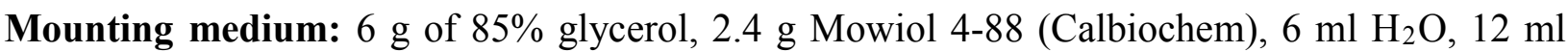
$0.2 \mathrm{M}$ Tris- $\mathrm{HCl} \mathrm{pH} 8.5$ and $2.5 \%$ DABCO

Cbc medium: BME [+] Earle's [-] L-Glutamine, 10\% Calf serum (heat inactivated, Hyclone), $1 \%$ PSG and $25 \mathrm{mM} \mathrm{KCl}$

BME112 medium: $35 \mathrm{mM}$ Glucose, $10 \mu \mathrm{g} / \mathrm{ml}$ Insulin, 1\% PSG in BME [+] Earle's [-] LGlutamine

2x HBSS: $4 \mathrm{~g} \mathrm{NaCl}, 117.5 \mathrm{mg} \mathrm{KCl}, 95 \mathrm{mg} \mathrm{Na}{ }_{2} \mathrm{HPO}_{4} \times \mathrm{xH}_{2} \mathrm{O}, 675 \mathrm{mg}$ glucose, $2.5 \mathrm{~g}$ Hepes-free acid in $250 \mathrm{ml} \mathrm{H}_{2} \mathrm{O}, \mathrm{pH} 7.05$ or $\mathrm{pH} 7.08$

HHGN: 1x HBSS-Hank's buffered salt solution [-] Calcium [-] magnesium, $2.5 \mathrm{mM}$ Hepes pH 7.3-7.5, $350 \mathrm{mM}$ glucose and $0.04 \mathrm{M} \mathrm{NaHCO}_{3}$

DnB: $500 \mu \mathrm{l}$ DNase $(2 \mu \mathrm{g} / \mu \mathrm{l}), 10 \mathrm{ml}$ BME [+] Earle's [-] L-Glutamine

TDn: $250 \mu \mathrm{l}$ DNase $(2 \mu \mathrm{g} / \mu \mathrm{l}), 5 \mathrm{ml}$ HHGN, $50 \mathrm{mg}$ Trypsin (Worthington)

Hippocampal neuron plating medium: DMEM $[+] 4.5 \mathrm{~g} / 1$ glucose $[+]$ glutamine $[+]$ pyruvate, $10 \%$ FBS (Biochrom), 1\% PSG, $0.0125 \mathrm{mM}$ glutamate

Neuron growth medium: Neurobasal medium, 2\% B27 supplement, 1\% PSG

HEK 293T medium: DMEM [+] $4.5 \mathrm{~g} / 1$ glucose [-] glutamine [-] pyruvate $\left(\mathrm{GIBCO}^{\circledR}\right.$, Life Technologies $^{\mathrm{TM}}$, Catalog no. 11960) with $10 \%$ FBS and 1\% GlutaMAX $^{\circledR}$

Bacterial lysis buffer: $10 \mathrm{mM}$ Tris/ $\mathrm{HCl} \mathrm{pH} 7.9,10 \%$ Glycerol, $0.5 \mathrm{M} \mathrm{NaCl}, 0.1 \%$ Nonidet P-40 and $5 \mathrm{mM}$ 2-Mercaptoethanol

BC100 buffer: $20 \mathrm{mM}$ Tris/HCl pH 7.9, 20\% Glycerol, $100 \mathrm{mM} \mathrm{KCl}$ and $5 \mathrm{mM} 2-$ Mercaptoethanol 
Tail Lysis buffer: $10 \mathrm{mM}$ Tris pH 8.0, $10 \mathrm{mM}$ EDTA, 0.5\% SDS; add fresh $200 \mathrm{mM} \mathrm{NaCl,} 200$ $\mu \mathrm{g} / \mathrm{ml}$ Proteinase K

Blocking buffer: BME [+] Earle's [-] L-Glutamine with $10 \% \mathrm{HS}$

DAPI: $1 \mu \mathrm{g} / \mu 1$ stock, dilute $1: 8000$ in $\mathrm{H}_{2} \mathrm{O}$

Hoechst: $1 \mu \mathrm{g} / \mu \mathrm{l}$ in PBS, $0.1 \%$ Triton X-100

X-Gal staining solution: $5 \mathrm{mM}$ potassium ferricyanide, $5 \mathrm{mM}$ potassium ferrocyanide, $2 \mathrm{mM}$ $\mathrm{MgCI}_{2}, 0.01 \%$ sodium deoxycholate, and $0.02 \%$ Nonidet P-40 (NP-40) in PBS, with freshly added $1 \mathrm{mg} / \mathrm{ml} \mathrm{X-Gal} \mathrm{(dissolved} \mathrm{in} \mathrm{DMSO)}$

\subsection{Expression plasmids and primers}

Expression constructs generated in the lab:

\begin{tabular}{|c|c|c|}
\hline Vector plasmid & Constructed by & Primers \\
\hline pBluescript-U6-RNF157 RNAi III & A. Matz & $14907 / 14908$ \\
\hline pCMV-myc-RNF157 & A. Matz & $15828 / 15829$ \\
\hline pCMV-myc-RNF157-Res & A. Matz & $22180 / 22181$ \\
\hline pCMV-myc-RNF157 $\Delta$ RING & A. Matz & $\begin{array}{l}15828 / 17434 / \\
17435 / 15829\end{array}$ \\
\hline pCMV-myc-Fe 65 aa $1-325$ & J. Stegmüller & $17802 / 17803$ \\
\hline pCMV-myc-Fe $65_{\text {aа326-524 }}$ & J. Stegmüller & $17804 / 17805$ \\
\hline pCMV-myc-Fe $65_{\text {aa } 525-711}$ & J. Stegmüller & $17806 / 17807$ \\
\hline pCMV-myc-Fe $65_{\text {aa } 1-524}$ & J. Stegmüller & $17802 / 17805$ \\
\hline pCMV-myc-Fe $65_{\text {aa326-711 }}$ & J. Stegmüller & $17804 / 17807$ \\
\hline pCMV-myc-Fe65 & A. Matz & $17802 / 17807$ \\
\hline pCMV-myc-NLS-FE65 & J. Stegmüller & \\
\hline pCMV-myc-NES-FE65 & J. Stegmüller & \\
\hline pBluescript-U6-Fe65 RNAi III & A. Matz & $19778 / 19779$ \\
\hline
\end{tabular}




\begin{tabular}{l|l|l} 
pEGFP-C2-RNF157 & A. Matz & $14864 / 14763$ \\
\hline pCMV-myc-Fe65 $\Delta$ PTB1 & S. Lee & $\begin{array}{l}17802 / 29386 / \\
29385 / 17807\end{array}$ \\
\hline pCMV-myc-Fe65 $\Delta$ PTB2 & S. Lee & $\begin{array}{l}17802 / 29384 / \\
29383 / 17807\end{array}$ \\
\hline pCMV-myc-Fe65 $\Delta$ B12 & S. Lee & $\begin{array}{l}17802 / 30092 / \\
30027 / 17807\end{array}$ \\
\hline pET3a-RNF157 immunogen & & $26304 / 26305$ \\
\hline pBluescript-U6-Tip110 RNAi \#1 & S. Lee & $31811 / 31812$ \\
\hline pBluescript-U6-Tip110 RNAi \#2 & S. Lee & $31813 / 31814$ \\
\hline pBluescript-U6-Tip110 RNAi \#3 & S. Lee & $31815 / 31816$ \\
\hline pBluescript-U6-Tip110 RNAi \#5 & S. Lee & $31819 / 31820$
\end{tabular}

Plasmids obtained from elsewhere:

\begin{tabular}{l|l} 
Plasmids & Obtained from \\
\hline pcDNA3.1-myc-Fe65 & U. Konietzko \\
\hline pcDNA3.1-Flag-Fe65 & T. Suzuki \\
\hline pcDNA3-human APP & A. Matz \\
\hline pcDNA3-swedish mutant APP & A. Matz \\
\hline pBICEP-CMV2-Tip60 & A. Repouskou \\
\hline pCS2-myc & M. Rape \\
\hline pCS2-myc-Tip110 & M. Rape \\
\hline pCS2-HA & M. Rape \\
\hline pCS2-HA-Tip110 & M. Rape
\end{tabular}


Sequence of the primers used for cloning:

\begin{tabular}{|c|c|}
\hline Primer & Sequence 5'-3' \\
\hline 14763 & GGCGTCGACCAGACAGCCAAAGGATCCCATACG \\
\hline 14864 & GGCAAGCTTCATGGGAGGAGAGAAGTTTGACTGC \\
\hline 14907 & $\begin{array}{l}\text { GGACAATAAGCTGTGCTCTGAAGTTAACGCAGAGCACAGCTTATTGTC } \\
\text { CCTTTTG }\end{array}$ \\
\hline 14908 & $\begin{array}{l}\text { AATTCAAAAGGGACAATAAGCTGTGCTCTGCGTTAACTTCAGAGCACA } \\
\text { GCTTATTGTCC }\end{array}$ \\
\hline 15828 & TATCTCGAGCCATGGGAGGAGAGAAGTTTGACTGC \\
\hline 15829 & TATGCGGCCGCTCAGACAGCCAAAGGATCCCATAC \\
\hline 17434 & GCCCGGAAGGGCAGCCGACACTCCGCACTGTTGTCACTGAC \\
\hline 17435 & GTCAGTGACAACAGTGCGGAGTGTCGGCTGCCCTTCCGGGC \\
\hline 17444 & TATAAGCTTCAACCTCACGCTGTCCTCCTCAG \\
\hline 17728 & TATTCTAGATCACGCACTGTTGTCACTGACATCATCC \\
\hline 17729 & TATTCTAGATCACTCACTCTCTGGAGTCACATCAGG \\
\hline 17730 & GGCTCTAGATCAGACAGCCAAAGGATCCCATACG \\
\hline 17742 & TATAAGCTTCGAGTGTGTGGTGTGTCTCTCAGAC \\
\hline 17802 & ATAGTCGACAATGTCTGTTCCATCATCACTGAG \\
\hline 17803 & ATAGCGGCCGCTTAGGCCTCATCACTGGGTTC \\
\hline 17804 & ATAGTCGACACCAATGGAGCTGGGACTG \\
\hline 17805 & ATAGTCGACACCAATGGAGCTGGGACTG \\
\hline 17806 & ATAGTCGACAGATGTCCCTTTCCAAGTGGA \\
\hline 17807 & ATAGCGGCCGCTCATGGGGTATGGGCCC \\
\hline 19778 & $\begin{array}{l}\text { AAGCTGACCCAGATGCTCAAAAGTTAACGTTGAGCATCTGGGTCAGCT } \\
\text { TCTTTTG }\end{array}$ \\
\hline 19779 & $\begin{array}{l}\text { AATTCAAAAGAAGCTGACCCAGATGCTCAACGTTAACTTTTGAGCATC } \\
\text { TGGGTCAGCTT }\end{array}$ \\
\hline 22180 & $\begin{array}{l}\text { GTCGCGAGCGTGAAAGCACTGGATAACAAACTCTGTTCGGAGGTCTGC } \\
\text { TTACCCGGTACCTGGCAAC }\end{array}$ \\
\hline 22181 & $\begin{array}{l}\text { GTTGCCAGGTACCGGGTAAGCAGACCTCCGAACAGAGTTTGTTATCCA } \\
\text { GTGCTTTCACGCTCGCGAC }\end{array}$ \\
\hline
\end{tabular}




\begin{tabular}{l|l}
$\mathbf{2 6 3 0 4}$ & TATAGAATTCGAGGAAGAGGATGGATCACC \\
\hline $\mathbf{2 6 3 0 5}$ & TATAGCGGCCGCCTACTGGGCATTCCGACTGAC \\
\hline $\mathbf{2 9 3 8 3}$ & GAATTCCCAGCGCCTAAGAATGCCTCCACCTCCTGC \\
\hline $\mathbf{2 9 3 8 4}$ & GCAGGAGGTGGAGGCATTCTTAGGCGCTGGGAATTC \\
\hline $\mathbf{2 9 3 8 5}$ & CCCCCACGGAATACCAACGTAAATGGACTCTCCCTGGA \\
\hline $\mathbf{2 9 3 8 6}$ & TCCAGGGAGAGTCCATTTACGTTGGTATTCCGTGGGGG \\
\hline $\mathbf{3 0 0 2 7}$ & CGTAATGCCCGCTGCTTGGAGTTGGTCCAGAAGTTCCAA \\
\hline $\mathbf{3 0 0 9 2}$ & TTGGAACTTCTGGACCAACTCCAAGCAGCGGGCATTACG \\
\hline $\mathbf{3 1 8 1 1}$ & $\begin{array}{l}\text { AAGAGCTCTGGCTGGAGTGGAAGTTAACGCCACTCCAGCCAGAGCTCT } \\
\text { TCTTTTG }\end{array}$
\end{tabular}

Sequence of primers for RNF157 genotyping:

RNF157 Genotyping Primer

\begin{tabular}{c|l|c}
\hline Primer & \multicolumn{1}{|c|}{ Sequence 5'-3, } & Description \\
\hline $\mathbf{2 8 7 4 3}$ & GAGAAAAGCAAGAGAAAGAGAAAAGC & WT FWD \\
\hline $\mathbf{2 8 7 4 4}$ & AGGCCATCTAAATACACGCACG & WT REV \\
\hline $\mathbf{2 5 1 7 1}$ & CTTGCAAAATGGCGTTACTTAAGC & Mut FWD \\
\hline $\mathbf{2 5 1 7 2}$ & GCGCTGACATCCTGTGTTC & Mut REV
\end{tabular}

\subsection{Antibodies}

Primary and secondary antibodies used for immunoblotting and immunostaining in this study:

\begin{tabular}{l|l|l|l|l}
\multirow{2}{*}{ Primary antibody } & \multirow{2}{*}{$\begin{array}{c}\text { Host } \\
\text { species }\end{array}$} & \multicolumn{1}{|c}{ Company } & \multicolumn{2}{|c}{ Working Dilution } \\
\cline { 4 - 5 } & Rabbit & Sigma-Aldrich & $1: 300$ & ICC/IHC \\
\hline$\alpha$ RNF157 & Mouse & Santa Cruz & $1: 1000$ & \\
\hline$\alpha 14-3-3 \beta$ & Rabbit & Santa Cruz & $1: 500$ & \\
\hline$\alpha$ Sp1 & Rabbit & Santa Cruz & $1: 500$ &
\end{tabular}




\begin{tabular}{l|l|l|l|l}
$\alpha \gamma$-Tubulin & Mouse & & $1: 5000$ & \\
\hline$\alpha \alpha$-Tubulin & Mouse & & $1: 5000$ & \\
\hline$\alpha$ myc & Mouse & Santa Cruz & $1: 1000$ & $1: 250$ \\
\hline$\alpha \beta$-galactosidase & Mouse & Santa Cruz & $1: 500$ & $1: 100$ \\
\hline$\alpha$ GFP & Rabbit & Invitrogen & $1: 1000$ & $1: 1000$ \\
\hline$\alpha$ GFP & Mouse & Santa Cruz & $1: 1000$ & \\
\hline$\alpha$ Fe65 & Rabbit & Santa Cruz & $1: 1000$ & \\
\hline$\alpha$ Fe65 & Goat & Santa Cruz & $1: 500$ & \\
\hline$\alpha$ Flag & Mouse & Sigma Aldrich & $1: 1000$ & $1: 250$ \\
\hline$\alpha$ Ubiquitin & Rabbit & Dako & $1: 500$ & \\
\hline$\alpha$ HA & Rabbit & Abcam & $1: 1000$ & $1: 200$ \\
\hline$\alpha$ HA & Mouse & Santa Cruz & $1: 500$ & \\
\hline$\alpha$ TuJ1 & Mouse & Promega & & $1: 250$ \\
\hline$\alpha$ N-Cadherin & Mouse & Becton Dickinson & $1: 3000$ & \\
\hline$\alpha$ & & & \\
\hline$\alpha$
\end{tabular}

\begin{tabular}{l|l|l|l|l|l}
\multirow{2}{*}{$\begin{array}{c}\text { Secondary } \\
\text { antibody }\end{array}$} & Host & Conjugated & \multirow{2}{*}{$\begin{array}{c}\text { Company } \\
\text { species }\end{array}$} & substrate/dye & \multicolumn{2}{|c}{ Working Dilution } \\
\cline { 5 - 6 } & Go mouse IgG & HRP & Dianova & $1: 10000$ & \\
\hline$\alpha$ rabbit IgG & Goat & HRP & Dianova & $1: 10000$ & \\
\hline$\alpha$ mouse IgG & Goat & Cy2/ Alexi 488 & Dianova & & $1: 1000$ \\
\hline$\alpha$ rabbit IgG & Goat & Cy2/ Alexa 488 & Dianova & & $1: 1000$ \\
\hline$\alpha$ mouse IgG & Goat & Cy3/ Alexa 555 & Dianova & & $1: 1000$ \\
\hline$\alpha$ rabbit IgG & Goat & Cy3/ Alexa 555 & Dianova & & $1: 1000$
\end{tabular}




\subsection{Expression plasmid construction and cloning}

\subsubsection{General molecular cloning}

To clone a desired cDNA sequence into an expression vector, the amplification setup is listed as in the table below. The annealing temperature was set $5^{\circ} \mathrm{C}$ below the melting temperature of the primers. For the PCR program, the elongation time at $72^{\circ} \mathrm{C}$ was set according to the length of the template ( $1 \mathrm{~min} / 500 \mathrm{bp}$ of template).

\section{PCR reaction setup}

\begin{tabular}{l|l}
\hline $1 \mu \mathrm{l}$ & template DNA $(10 \mathrm{ng} / \mu \mathrm{l})$ \\
\hline $1 \mu \mathrm{l}$ & forward primer $(10 \mathrm{pmol} / \mu \mathrm{l})$ \\
\hline $1 \mu \mathrm{l}$ & reverse primer $(10 \mathrm{pmol} / \mu \mathrm{l})$ \\
\hline $0.4 \mu \mathrm{l}$ & dNTPs $(25 \mathrm{mM})$ \\
\hline $0.5 \mu \mathrm{l}$ & Pfu DNA polymerase $(2.5$ units $/ \mu \mathrm{l})$ \\
\hline $5 \mu 1$ & $10 X$ reaction buffer $\left(+\mathrm{MgSO}_{4}\right)$ \\
\hline $41.1 \mu \mathrm{l}$ & $\mathrm{H}_{2} \mathrm{O}$
\end{tabular}

PCR Program

\begin{tabular}{l|l|l}
\hline Denaturation $95^{\circ} \mathrm{C}$ & $3 \mathrm{~min}$ & \\
\cline { 1 - 2 } Denaturation $95^{\circ} \mathrm{C}$ & $30 \mathrm{sec}$ & \multirow{3}{*}{30 cycles } \\
\cline { 1 - 2 } Annealing $\mathrm{X}^{\circ} \mathrm{C}$ & $30 \mathrm{sec}$ & \\
\cline { 1 - 2 } Elongation $72^{\circ} \mathrm{C}$ & $\mathrm{X} \min$ & \\
\cline { 1 - 2 } Final elongation $72^{\circ} \mathrm{C}$ & $5 \mathrm{~min}$ &
\end{tabular}

To assess size and quality of the PCR product, the product was subjected to electrophoresis in a $0.8-2 \%$ agarose gel according to the expected size. DNA fragments of correct size were excised from the gel, extracted with NucleoSpin ${ }^{\circledR}$ Gel and PCR Clean-up kit and eluted in $40 \mu 1$ $\mathrm{H}_{2} \mathrm{O}$.

The purified PCR product (insert) and the target expression vector $(2 \mu \mathrm{g})$ were digested with the corresponding restriction enzymes for $1-2$ hour at $37^{\circ} \mathrm{C}$ in a volume of $50 \mu \mathrm{l}$. Directly after digestion, the insert was purified using NucleoSpin ${ }^{\circledR}$ Gel and PCR Clean-up kit and eluted in 20 $\mu \mathrm{H} \mathrm{H}_{2} \mathrm{O}$. The digested vector was further treated with 10 units of calf intestinal alkaline phosphatase (CIP) for $30 \mathrm{~min}$ at $37^{\circ} \mathrm{C}$ to remove the $5^{\prime}$ phosphate groups of DNA and thus prevent self-religation. After CIP treatment, the vector was separated electrophoretically in an 
agarose gel to verify the DNA linearization. The linearized fragment was subsequently extracted with NucleoSpin ${ }^{\circledR}$ Gel and PCR Clean-up kit and in $40 \mu 1 \mathrm{H}_{2} \mathrm{O}$.

For the ligation of the digested insert and vector, the reaction setup is listed in the table below:

\section{Ligation reaction setup}

\begin{tabular}{l|l}
\hline $6 \mu 1$ & insert \\
\hline $1 \mu 1$ & vector \\
\hline $1 \mu 1$ & ATP $(10 \mathrm{mM})$ \\
\hline $1 \mu 1$ & $10 \mathrm{X}$ T4 ligation buffer \\
\hline $1 \mu 1$ & T4 DNA ligase \\
\hline
\end{tabular}

The next day, the entire ligation reaction was transferred into $100 \mu \mathrm{l}$ of chemically competent DH5 $\alpha$ E. coli cells. The bacteria were then incubated on ice for $20 \mathrm{~min}$, heat shocked at $37^{\circ} \mathrm{C}$ for $1 \mathrm{~min}$ and cooled down on ice for $1 \mathrm{~min}$. Afterwards, $300 \mu \mathrm{l}$ antibiotic-free $2 \mathrm{xYT}$ media (room temperature) was added into the reaction followed by $30 \mathrm{~min}$ of shaking at $37^{\circ} \mathrm{C}$. Next, bacteria were spun down at $4000 \mathrm{rpm}$ for $3 \mathrm{~min}$ and plated on a pre-warmed 2xYT agar plate with a suitable antibiotic according to the resistance gene encoded in the vector. The plate was kept at $37^{\circ} \mathrm{C}$ overnight.

To assess if the ligation was successful, colonies on the plate were transferred into $2 \mathrm{ml}$ 2xYT media supplemented with the corresponding antibiotic. The cultures were incubated overnight at $37^{\circ} \mathrm{C}$. Next, the plasmid DNA from each culture was extracted using the NucleoSpin ${ }^{\circledR}$ Plasmid Quick Pure kit. The plasmids were then digested with the appropriate enzymes to determine the positive clones. Afterwards, the sequence of the DNA from positive clones was analyzed by the AGCTLab at the MPI of Experimental Medicine.

\subsubsection{Construction of vector-based RNA interference}

In this study, the RNA interferences (RNAi) were constructed in the pBlueScript vector containing the RNA polymerase III U6 promoter. The rules listed below were use to design the primers for generating RNAi: 
1. 5' primer starts with $\mathrm{AA}, \mathrm{AG}$ or $\mathrm{GG}$

2. Followed by 18 nucleotides (target sequence), alternative loop sequence AAGTTAACG harboring an HpaI site and the antiparallel target sequence

3. End with CTTTG (stop signal for the RNA polymerase III and a part of EcoRI site)

4. The target sequence should have $45-60 \%$ GC content

5. Avoid AUG and AAAA

6. Confirm that the sequence is specific for the target DNA

7. The 3 ' primer is complementary to the 5' primer with TTAA added to the end

To generate the RNAi, $2 \mu 1$ of 5' sense and 3' antisense primer (50 pmol/ $\mu$ l) were resuspended in $46 \mu \mathrm{l}$ of annealing buffer, incubated at $95^{\circ} \mathrm{C}$ for $4 \mathrm{~min}, 72^{\circ} \mathrm{C}$ for $10 \mathrm{~min}$ and slowly cooled down $\left(0.1^{\circ} \mathrm{C} / \mathrm{sec}\right)$ to room temperature. $20 \mu \mathrm{l}$ of the annealed reaction mix was phosphorylated using polynucleotide kinase $(\mathrm{PNK})$ at $37^{\circ} \mathrm{C}$ for $30 \mathrm{~min}$. In the meanwhile, the pBlueScript-U6 vector was first digested with ApaI and then blunted with T4 DNA polymerase to remove the sticky ends of the vector. Further, the vector was digested with EcoRI and dephosphorylated by CIP. The annealed oligonucleotides (insert) and the vector were ligated overnight in ice-cold water and transformed into DH5 $\alpha$ E. coli cells as described in Section 2.7.1. Positive clones were tested using HpaI restriction enzyme since the HpaI site was designed into the primers. The sequence was analyzed by the AGCTLab.

\subsection{Cell culture and transfection}

All the experimental procedure concerning cell culture was done in a biological safety cabinet (HERAsafe ${ }^{\circledR}$, Kendro). Cultured cells were kept in appropriate media according to the cell types. All the heterologous cells were grown in an incubator (Hera cell 150, Kendro) supplemented with $5 \% \mathrm{CO}_{2}$ at $37^{\circ} \mathrm{C}$, while the primary cells were in another incubator to avoid contamination.

\subsubsection{Culture of HEK293T cells}

HEK293T cells were maintained in a 10-cm dish with HEK293T medium in the CO2Incubator. Cell splitting was performed when the cells reached $90 \%$ confluence. First, the cells were washed once with PBS and incubated with $2 \mathrm{ml}$ of $1 \mathrm{x}$ TE at $37^{\circ} \mathrm{C}$ for $2 \mathrm{~min}$. Next, $8 \mathrm{ml}$ of 
HEK293T medium was added into the plate and mixed with the cells by pipetting up and down. The cell suspension was then spun down at $800 \mathrm{rpm}$ for $5 \mathrm{~min}$ at $4^{\circ} \mathrm{C}$, resuspended in $5 \mathrm{ml}$ medium and plated in 6-well, 12 -well or $10-\mathrm{cm}$ plates according to the experiment design. For maintenance of the cell line, $500 \mu \mathrm{l}$ of cell suspension was added into $10 \mathrm{ml}$ medium and plated in a $10-\mathrm{cm}$ dish.

\subsubsection{Transfection of HEK293T cells}

HEK293T cells were split into a 6-well plate one day before transfection. When the cells grew to approx. $70-80 \%$ confluency, $10 \mathrm{ng}-2 \mu \mathrm{g}$ plasmid DNA was diluted into $90 \mu 1$ sterile $\mathrm{H}_{2} \mathrm{O}$. Next, the DNA solution was mixed properly with $10 \mu \mathrm{l}$ of $2.5 \mathrm{M} \mathrm{CaCl}_{2}$ and then with $100 \mu \mathrm{l}$ of $2 \times$ HBSS. The mixture was incubated for 5 min at RT and added gently to the cells.

\subsubsection{Culture of primary cerebellar granule neurons}

Isolated cerebella from postnatal day 6 (P6) Wistar rats or from P5 RNF157 transgenic mice were collected in HHGN medium on ice. Using fine forceps, the meninges were then removed under a dissection microscope. After 3 HHGN washes, the cerebella were incubated with $5 \mathrm{ml}$ of TDn for $10 \mathrm{~min}$ at $37^{\circ} \mathrm{C}$ and another $10 \mathrm{~min}$ at room temperature. Next, the cerebella were washed 3 times with HHGN medium and triturated in $4 \mathrm{ml}$ of DnB till the suspension reached homogeneity. After $5 \mathrm{~min}$ incubation on ice, the supernatant was transferred into a fresh falcon. The remaining tissue was triturated with another $4 \mathrm{ml}$ of $\mathrm{DnB}$ and combined with the separated supernatant. The cell suspension was centrifuged at $800 \mathrm{rpm}$ for $5 \mathrm{~min}$ at $4^{\circ} \mathrm{C}$ and the pellet was resuspended in $20 \mathrm{ml}$ of $\mathrm{Cbc}$ medium. The number of cells was estimated in a hemocytometer. Prior to plating, the plates or coverslips were coated with $15 \mu \mathrm{g} / \mathrm{ml}$ of poly-ornithine for at least $30 \mathrm{~min}$ at $37^{\circ} \mathrm{C}$ and washed twice with sterile $\mathrm{H}_{2} \mathrm{O} .20 \times 10^{6}$ cells were then seeded in a 24-well plate, $30 \times 10^{6}$ cells in a 6 -well plate and $50 \times 10^{6}$ cells in a $10-\mathrm{cm}$ dish. At the next day, day in vitro (DIV) 1, neurons were treated with an anti-mitotic agent, $10 \mu \mathrm{M}$ nucleoside cytosine arabinoside (AraC), to inhibit the proliferation of non-neuronal cells. If neurons were kept longer in culture, $35 \mathrm{mM}$ glucose was supplemented to the medium every third day. 


\subsubsection{Culture of primary cortical neurons}

Cortices were isolated from E18 Wistar rat embryos in 1 x HBSS medium (BME [-] calcium [-] magnesium, Gibco ${ }^{\circledR}$ ) under a dissection microscope and transferred to a falcon filled with HBSS medium on ice. After 3 HBSS washes, the cortices were incubated with $5 \mathrm{ml}$ of TDn for $10 \mathrm{~min}$ at $37^{\circ} \mathrm{C}$ and another $10 \mathrm{~min}$ at room temperature. Next, the tissues were washed 3 times with HBSS medium and triturated in $5 \mathrm{ml}$ of neuron growth medium with $250 \mu 1$ freshly added DNase $(2 \mathrm{mg} / \mathrm{ml})$. The cell suspension was centrifuged at $800 \mathrm{rpm}$ for $5 \mathrm{~min}$ at $4^{\circ} \mathrm{C}$ the pellet was resuspended in $20 \mathrm{ml}$ of neuron growth medium. Cells were counted in a hemocytometer. Prior to plating, the plates or coverslips were coated with $15 \mu \mathrm{g} / \mathrm{ml}$ of poly-ornithine for at least $30 \mathrm{~min}$ at $37^{\circ} \mathrm{C}$ and washed twice with sterile $\mathrm{H}_{2} \mathrm{O} \cdot 1.2-2 \times 10^{6}$ cells were seeded in a 24-well plate, $8 \times 10^{6}$ cells in a 6 -well plate and $10 \times 10^{6}$ cells in a $10-\mathrm{cm}$ dish.

\subsubsection{Culture of primary hippocampal neurons}

Hippocampi were isolated from E18 Wistar rat embryos in HBSS medium (BME [-] calcium [-] magnesium, Gibco ${ }^{\circledR}$ ) and transferred to a falcon filled with HBSS medium on ice. After 3 HBSS washes, the volume was reduced to $1.8 \mathrm{ml}$ and $200 \mu \mathrm{l}$ of $0.05 \%$ Trypsin-EDTA (Gibco ${ }^{\circledR}$ ) was added. The hippocampi were incubated for $10 \mathrm{~min}$ at $37^{\circ} \mathrm{C}$. Followed by another $3 \mathrm{HBSS}$ washes, the hippocampi were triturated in $1 \mathrm{ml}$ of hippocampal neuron plating medium with 50 $\mu 1 \mathrm{DNase}(2 \mathrm{mg} / \mathrm{ml})$. The cell suspension was centrifuged at $800 \mathrm{rpm}$ for $5 \mathrm{~min}$ at $4^{\circ} \mathrm{C}$ the pellet was resuspended in $5 \mathrm{ml}$ of hippocampal neuron plating medium. Cells were counted in a hemocytometer. Prior to plating, the plates or coverslips were coated with $0.00083 \%$ poly-LLysine for at least $30 \mathrm{~min}$ at $37^{\circ} \mathrm{C}$ and washed twice with sterile $\mathrm{H}_{2} \mathrm{O}$. 1.2-2 $\times 10^{6}$ cells were seeded in a $24-w e l l$ plate and $8 \times 10^{6}$ cells in a 6-well plate. In the next day, the plating medium was replaced by neuron growth medium.

\subsubsection{Transfection of primary neurons and nucleofection}

Primary cerebellar granule neurons, hippocampal neurons and cortical neurons in 24-well plates were transfected at the indicated points in time. First, the conditioned culture medium was collected and kept at $37^{\circ} \mathrm{C}$. Neurons were washed twice with prewarmed DMEM $([+] 4.5 \mathrm{~g} / 1$ glucose [-] glutamate [-] pyruvate for transfection) and starved for 30-45 min in $500 \mu 1$ DMEM 
at $37^{\circ} \mathrm{C}$. Meanwhile, the plasmid DNA was diluted in sterile $\mathrm{H}_{2} \mathrm{O}$ to a volume of $18 \mu \mathrm{l}$. Then, the DNA solution was mixed properly with $2 \mu \mathrm{l}$ of $2.5 \mathrm{M} \mathrm{CaCl}_{2}$ and then with $20 \mu \mathrm{l}$ of $2 \times \mathrm{HBSS}$. The mixture was incubated for at least $5 \mathrm{~min}$ at RT and added gently to the cells after cell starvation. The neurons were incubated at $37^{\circ} \mathrm{C}$ for $5-18 \mathrm{~min}$ according to the experimental setup. Finally, neurons were washed twice with DMEM and the collected conditioned medium returned.

For nucleofection, 4-8 $\times 10^{6}$ dissociated cortical neurons were resuspended in $100 \mu \mathrm{l}$ of DMEM ([+] $4.5 \mathrm{~g} / 1$ glucose [-] glutamate [-] pyruvate) and mixed with $8 \mu \mathrm{g}$ of the indicated RNAi plamids, together with the GFP plasmid and the anti-apoptotic Bcl-xL plasmid. The cell suspension was pulsed using the program O-005 in the nucleofector (Amaxa, Lonza). Neurons were then immediately transferred into pre-warmed DMEM supplemented with $10 \%$ FCS in 6well plates. After 4 hours, the medium was exchanged with neuron growth medium.

\subsection{Immunocytochemistry}

The cells for morphological analysis were washed twice with PBS and fixed with 4\% PFA for $10 \mathrm{~min}$, while the neurons for survival assay were directly fixed by adding $8 \%$ PFA to the medium. After 2 more PBS washes, cells were permeabilized with 0.4\% Triton X-100 in PBS for $10 \mathrm{~min}$. Cells were then washed twice with PBS followed by blocked in blocking buffer (BME + $10 \%$ horse serum) for $30 \mathrm{~min}$. Next, primary antibody diluted in blocking buffer was applied to cells for either 1 hour at RT or overnight at $4^{\circ} \mathrm{C}$. Afterwards, the cells were washed twice with blocking buffer before the secondary antibody, the fluorophore-coupled antibody was diluted in blocking buffer and added to the cells. After $45 \mathrm{~min}$ of incubation, cells were washed twice with PBS and incubated with a DNA dye (bisbenzimide Hoechst 33258 or 4'6-diamidino-2phenylindole - DAPI) for 5-10 min. After 2 PBS washes, coverslips were mounted upside down with Mowiol mounting medium on microscope slides (Marienfeld) and kept at $4{ }^{\circ} \mathrm{C}$ till analysis.

\subsubsection{Survival assays in neurons}

Procedures for survival assays were modified from a previously described method (Becker and Bonni, 2004). Briefly, neurons were prepared as mentioned in 2.8.3 and 2.8.5 and seeded in a 24-well plate. At DIV2, neurons were transfected with the indicated expression plasmids together with a plasmid encoding $\beta$-galactosidase using the calcium phosphate transfection method as in 2.8.6. For gain-of-function experiments, neurons were cultured for 6 days, while 7 days for loss-of-function or epistasis analyses. Next, neurons were subjected to immunocytochemistry using the monoclonal $\beta$-galactosidase antibody and abovementioned 
DNA dyes. Neuronal survival and death were examined in $\beta$-galactosidase expressing neurons based on the integrity of neurites and the morphology of the nucleus. Analyses were performed in a blinded manner $(\mathrm{n} \approx 100$ cells per condition) using a fluorescence microscope.

\subsection{X-Gal staining of mouse brain sections}

For the sagittal brain sections, mice were first perfused with 4\% PFA and the brains were put into $4 \%$ PFA, gently shake at $4{ }^{\circ} \mathrm{C}$ for 3 hours as post-fixation. Afterwards, the brains were rinsed twice with PBS and kept in PBS supplemented with $0.02 \% \mathrm{NaN}_{3}$ at $4{ }^{\circ} \mathrm{C}$ till cutting. Dissection was performed using a vibratom (VT1000S, Leica). The brains were cut into $40 \mu \mathrm{m}$ sections and stored in PBS with $\mathrm{NaN}_{3}$.

For staining, sections were incubated with $\mathrm{X}$-Gal staining solution under lightproof conditions overnight at $37 \%$. The next day, the sections were washed twice with PBS, collected on a slide and stored at $4 \%$. The images were obtained using an inverted light microscope.

\subsection{Biochemical methods}

\subsubsection{Tissue lysate preparation}

Mice or rats were sacrificed by either decapitation or $\mathrm{CO}_{2}$ inhalation. Tissues were isolated and placed rapidly into ice-cold TX-100 lysis buffer with freshly added protease inhibitor cocktail ( $1 \mathrm{mM}$ DTT, $3 \mu \mathrm{g} / \mathrm{ml}$ aprotinin, $1 \mu \mathrm{g} / \mathrm{ml}$ leupeptin and $1 \mu \mathrm{g} / \mathrm{ml}$ pepstatin). Subsequently, the mixture was homogenized using $2 \mathrm{ml}$ Dounce or Ultra Turrax $\left(\mathrm{IKA}^{\circledR}\right)$ homogenizer. After 30 min of incubation on ice, samples were spun down at $14000 \mathrm{rpm}$ for $10 \mathrm{~min}$ at $4^{\circ} \mathrm{C}$ and the supernatants were collected into a fresh eppendorf tube. If not immediately used for further experiments, the lysates were flash frozen in liquid nitrogen and kept at $-80^{\circ} \mathrm{C}$.

\subsubsection{Cell lysate preparation}

To collect the lysate from cultured HEK 293 T or primary neurons, cultured medium was first aspirated off from the plates and the cells were washed once with PBS on the ice. Next. TX100 lysis buffer with freshly added protease inhibitor cocktail (1 mM DTT, $3 \mu \mathrm{g} / \mathrm{ml}$ aprotinin, 1 $\mu \mathrm{g} / \mathrm{ml}$ leupeptin and $1 \mu \mathrm{g} / \mathrm{ml}$ pepstatin) was added to the wells. Adherent cells were then 
removed from the plates using a cell scraper and collected in an eppendorf tube. Samples were incubated on ice for $30 \mathrm{~min}$ and centrifuged at $14000 \mathrm{rpm}$ for $10 \mathrm{~min}$ at $4^{\circ} \mathrm{C}$. The supernatants were collected into a fresh tube and kept at $4^{\circ} \mathrm{C}$ for the further experiments.

\subsubsection{Bradford protein assay}

To determine the concentration of protein in tissue or cell lysates, Bradford Reagent (Bio$\operatorname{Rad}^{\circledR}$ ) was used. The Reagent was diluted 5x in PBS. For the standard curve, 2, 4, and $7 \mu \mathrm{g}$ of

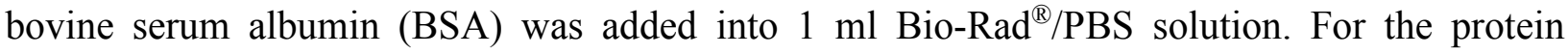
concentration, $2 \mu \mathrm{l}$ of lysates from proteins of interest were suspended in $1 \mathrm{ml} \mathrm{Bio-Rad}{ }^{\circledR} / \mathrm{PBS}$ solution. The absorbance shift based on the protein binding to Coomassie Brilliant Blue G-250 was determined by a photometer (Amersham Biosciences, Ultro spec 3100 pro) at the wavelength of $595 \mathrm{~nm}$. Lastly, the protein concentration was calculated according to the BSA standard curve.

\subsubsection{Co-Immonoprecipitation (CoIP)}

Transfected HEK 293T were washed once with PBS and lyzed in ice-cold CoIP buffer with freshly added protease inhibitor cocktail ( $1 \mathrm{mM} \mathrm{DTT}, 3 \mu \mathrm{g} / \mathrm{ml}$ aprotinin, $1 \mu \mathrm{g} / \mathrm{ml}$ leupeptin and 1 $\mu \mathrm{g} / \mathrm{ml}$ pepstatin) on ice. Samples were incubated on ice for $30 \mathrm{~min}$ and centrifuged at $14000 \mathrm{rpm}$ for $10 \mathrm{~min}$ at $4^{\circ} \mathrm{C}$. After measuring the protein concentration, $100 \mu \mathrm{g}$ of the samples were set aside as input control. Then, at least $1 \mathrm{mg}$ of protein was incubated with $1 \mu \mathrm{g}$ of antibody for 3 hours at $4^{\circ} \mathrm{C}$ on the rotator. Meanwhile, protein A-sepharose beads (Protein A-Sepharose ${ }^{\mathrm{TM}}$ Fast Flow, GE Healthcare), which bind to rabbit and mouse IgGs, were washed 3 times with CoIP buffer and stored in equal amount of CoIP buffer (i.e. $300 \mu 1$ buffer for $300 \mu \mathrm{l}$ beads) till needed. After 3 hours, $30 \mu \mathrm{l}$ of washed beads was added to every sample and rotated for another hour at $4^{\circ} \mathrm{C}$. Next, samples were spun down at $10000 \mathrm{rpm}$ for $1 \mathrm{~min}$ and the supernatants were discarded. The beads were washed twice with either RIPA or TX-100 lysis buffer and once with PBS. Finally, the PBS was removed leaving $\sim 10 \mu \mathrm{l}$ of the liquid upon the beads. The samples were then boiled at $95^{\circ} \mathrm{C}$ for $5 \mathrm{~min}$ with $20 \mu \mathrm{l}$ of $4 \mathrm{x}$ SDS sample buffer. The boiled CoIP samples and input controls were further subjected to SDS-PAGE and western-blot analysis. 


\subsubsection{Subcellular fractionation}

Primary neurons were grown in a 10-cm plate. Neurons were first washed once with PBS and lyzed in $250 \mu$ l of buffer A with freshly supplemented protease inhibitors $(5-10 \mu \mathrm{g} / \mathrm{ml}$ aprotinin, $1 \mathrm{mM}$ PMSF and 5-10 mM N-Ethylmaleimide (NEM). If mouse brains were used, brain tissues were lyzed directly in Buffer A with protease inhibitors. Next, samples were kept 15 $\mathrm{min}$ on ice and subjected to a mechanical disruption using a $2 \mathrm{ml}$ Dounce homogenizer.

When lysates reached homogeneity, samples were centrifuged at $2000 \mathrm{rpm}$ for $5 \mathrm{~min}$ at $4^{\circ} \mathrm{C}$. The postnuclear supernatant (PNS) was collected in a new eppendorf tube as the cytoplasmic fraction. The remaining nuclear pellets were then washed three times with Buffer $\mathrm{A}^{+}$and resuspended in $50 \mu \mathrm{l}$ Buffer $\mathrm{C}$ followed by $30 \mathrm{~min}$ of rotation at $4^{\circ} \mathrm{C}$. In the last step, the nuclei were centrifuged at $14000 \mathrm{rpm}$ for $20 \mathrm{~min}$ at $4^{\circ} \mathrm{C}$ and the supernatant was collected as nuclear fraction (NF).

For the tissue lysate, samples were centrifuged at $2000 \mathrm{rpm}$ for $5 \mathrm{~min}$ at $4^{\circ} \mathrm{C}$. The supernatant (S1) was collected in a new eppendorf tube and spun down again for 5 min at $4^{\circ} \mathrm{C}$. The supernatant from S1 was then the PNS. The pellets from S1 were washed 6 times with Buffer $\mathrm{A}^{+}$, resuspended in $100 \mu \mathrm{l}$ Buffer $\mathrm{C}$ and rotated for $30 \mathrm{~min}$ at $4^{\circ} \mathrm{C}$. Finally, the pellets were spun down at $14000 \mathrm{rpm}$ for $20 \mathrm{~min}$ at $4^{\circ} \mathrm{C}$ and the supernatant was harvested as nuclear fraction (NF). For further fractionation, the PNS I was ultracentrifuged for $40 \mathrm{~min}$ at $4^{\circ} \mathrm{C}$ at $21000 \mathrm{~g}$, the resulting pellet I and PNS II were collected. PNS II was then ultracentrifuged for 2.5 hours at $4^{\circ} \mathrm{C}$ at $257000 \mathrm{~g}$ resulting in pellet II and the cytoplasmic fraction. After measuring the protein concentration, equal amount of PNS and NF was subjected to SDS-PAGE and western-blot analysis.

\subsubsection{Ubiquitination assay}

Ubiquitination assays were performed under denaturing conditions according to the protocol of $\mathrm{Lu}$ and colleagues with some modifications to avoid the detection of non-specific ubiquitination ( $\mathrm{Lu}$ et al., 2007). Briefly, transfected HEK 293T cells were lysed in RIPA buffer without SDS (50 mM Tris- $\mathrm{HCl} \mathrm{pH} 8.0,150 \mathrm{mM} \mathrm{NaCl}, 1 \% \mathrm{NP} 40,0.5 \%$ sodium deoxycholate, 5 $\mathrm{mM}$ EDTA, protease inhibitors and $10 \mathrm{mM} \mathrm{NEM}$ ). Next, $1 \mathrm{mg}$ of total protein was incubated in $1 \%$ SDS for $5 \mathrm{~min}$ at $4{ }^{\circ} \mathrm{C}$, boiled for $12.5 \mathrm{~min}$ at $95{ }^{\circ} \mathrm{C}$, diluted $10 \mathrm{x}$ in lysis buffer $(50 \mathrm{mM}$ HEPES $\mathrm{pH} 7.5,150 \mathrm{mM} \mathrm{NaCl}, 10 \%$ glycerol, $1.5 \mathrm{mM} \mathrm{MgCl}_{2}, 1 \%$ Triton X-100) to adjust the concentration of SDS to $0.1 \%$ and immunoprecipitated with an antibody or with antibodyconjugated beads overnight at $4{ }^{\circ} \mathrm{C}$. For non-conjugated antibodies, the antibody-antigen complex 
was immunoprecipitated for $45 \mathrm{~min}$ at $4^{\circ} \mathrm{C}$ using Protein A-Sepharose. Precipitated proteins bound to the beads were washed twice with lysis buffer, twice with HNTG buffer $(20 \mathrm{~mm}$ HEPES, pH 7.5, $150 \mathrm{~mm} \mathrm{NaCl}, 0.1 \%$ Triton X-100, 10\% Glycerol) and eluted by boiling the beads in SDS sample buffer. Lysates were analyzed by SDS-PAGE and subsequent immunoblotting analysis.

\subsubsection{SDS-PAGE and western blot}

For SDS-PAGE, the polyacrylamide gels were prepared in the gel casting system (MiniPROTEAN Tetra Electrophoresis System, Bio-Rad ${ }^{\circledR}$ ). A gel consists of a lower separating gel (8$15 \%$ of acrylamide according to experimental design) and an upper stacking gel (3.9\%). The most commonly used gels contained $8 \%, 10$ or $12 \%$ and the reagents needed are listed below:

\begin{tabular}{l|c|c|c||c} 
& \multicolumn{3}{|l|}{ Separating Gel (ml) } & Stacking Gel \\
\hline \% Acrylamide & $8 \%$ & $10 \%$ & $12 \%$ & $3.9 \%$ \\
\hline $30 \%$ Acrylamide & 2 & 2.5 & 3 & $0.65 \mathrm{ml}$ \\
\hline Lower buffer & 1.875 & 1.875 & 1.875 & $1.25 \mathrm{ml}$ Upper buffer \\
\hline $\mathrm{H}_{2} \mathrm{O}$ & 3.625 & 3.125 & 2.625 & $3.05 \mathrm{ml}$ \\
\hline APS & \multicolumn{3}{|c}{$30 \mu 1$} \\
\hline TEMED & \multicolumn{3}{|l}{$3 \mu 1$}
\end{tabular}

The reagents for a separating gel were first mixed, loaded into the gel system and covered by a layer of isopropanol. After 45 min of polymerization, isopropanol was washed out with $\mathrm{H}_{2} \mathrm{O}$ and the stacking gel was added to the top followed by the insert of a 10 -well comb. The upper gel was polymerized after about $40 \mathrm{~min}$.

Lysates containing 30-80 $\mu \mathrm{g}$ of proteins were diluted in $4 \times$ SDS sample buffer and boiled for $5 \mathrm{~min}$ at $95^{\circ} \mathrm{C}$. After loading into the gel, the proteins were separated by eletrophoresis at $35 \mathrm{~mA}$ per gel. Subsequently, the proteins were transferred onto a nitrocellulose membrane using a Mini Trans-Blot ${ }^{\circledR}$ cell $\left(\right.$ Bio-Rad $\left.^{\circledR}\right)$ at 250-300 mA for $90 \mathrm{~min}$. After transfer, the membrane was blocked in PBST supplemented with 4\% milk powder (Frema Reform) for $30 \mathrm{~min}$ at RT and washed 3 times for 10 min in PBST. Next, a primary antibody prepared in 3\% BSA or 4\% milk powder with $0.02 \% \mathrm{NaN}_{3}$ in PBST was added to the membrane and incubated for 1 hour at RT or overnight at $4^{\circ} \mathrm{C}$. Afterwards, the membrane was washed 3 times with PBST for 10 min and 
incubated with a secondary antibody diluted in PBST with 4\% milk powder for 30-45 min at RT. The secondary antibody was coupled with horseradish peroxidase (HRP). Lastly, the membrane was washed 3 times with PBST for 10 min and incubated shortly in enhanced chemiluminescent (ECL) horseradish peroxidase substrates (Thermo Fisher Scientific). An X-ray film was then exposed to the membrane and developed in an automatic film processor (Kodak imaging station).

\subsubsection{Protein expression and purification}

The cDNA of interest was first cloned into pET3a expression vector. Then, the plasmid was transformed into BL21 E. coli cells on a 2xYT agar plate. A single colony was used for a $20 \mathrm{ml}$ overnight pre-culture in $2 \mathrm{xYT}$ medium supplemented with ampicillin at $37^{\circ} \mathrm{C}$. Next day, appropriate amount of the pre-culture was diluted in $250 \mathrm{ml}$ of $2 \mathrm{xYT}$ supplemented with ampicillin until the $\mathrm{OD}_{600}$ was 0.1 . Then, the culture was incubated on a shaker for $2.5 \sim 3$ hours at $37^{\circ} \mathrm{C}$ and $1 \mathrm{mM}$ IPTG was added to the culture when the $\mathrm{OD}_{600}$ reached $0.5 \sim 0.6$. Afterwards, the culture was shaken for another $3 \sim 3.5$ hours at $30^{\circ} \mathrm{C}$, distributed into $50-\mathrm{ml}$ falcons and spun down at $3500 \mathrm{rpm}$ for $10 \mathrm{~min}$ at $4^{\circ} \mathrm{C}$. The pellets were lyzed in ice-cold bacterial lysis buffer with freshly added protease inhibitors ( $1 \mu \mathrm{g} / \mathrm{ml}$ leupeptin, $1 \mu \mathrm{g} / \mathrm{ml}$ pepstatin and $0.5 \mathrm{mM}$ PMSF). Next, the samples were sonicated at $4{ }^{\circ} \mathrm{C}$ using $30 \%$ of the power and for 3 times $(30 \mathrm{sec}$ each sonication with $30 \mathrm{sec}$ intersection interval). After sonication, the samples were centrifuged at $15000 \mathrm{~g}$ for $30 \mathrm{~min}$ at $4^{\circ} \mathrm{C}$. The supernatant was collected into a new falcon with $1 \mathrm{mM}$ immidazol, $1 \mathrm{mM}$ PMSF and $500 \mu \mathrm{Ni}$-sepharose beads and rotated overnight at $4^{\circ} \mathrm{C}$.

Next day, the sample was spun down at $4000 \mathrm{rpm}$ for $5 \mathrm{~min}$ at $4^{\circ} \mathrm{C}$ and the supernatant was collected as flow through (FT). $10 \mathrm{ml} \mathrm{BC100} \mathrm{buffer} \mathrm{supplemented} \mathrm{with} 20 \mathrm{mM}$ immidazol and $0.5 \mathrm{mM}$ PMSF was added to the beads and the sample was rotated for $30 \mathrm{~min}$ at $4{ }^{\circ} \mathrm{C}$. Then, the sample was again centrifuged at $4000 \mathrm{rpm}$ for $5 \mathrm{~min}$ at $4{ }^{\circ} \mathrm{C}$ and the supernatant was kept as wash I (WI). The procedure was repeated with $5 \mathrm{ml} \mathrm{BC100}$ buffer supplemented with $40 \mathrm{mM}$ immidazol and 0.5 mM PMSF for wash II (WII). Finally, $400 \mu \mathrm{BC} 100$ buffer together with $200 \mathrm{mM}$ immidazol and $0.5 \mathrm{mM}$ PMSF was added to the beads. After $30 \mathrm{~min}$ rotation at $4^{\circ} \mathrm{C}$, the sample was spun down at $4000 \mathrm{rpm}$ for $5 \mathrm{~min}$ at $4{ }^{\circ} \mathrm{C}$ and the supernatant was the purified protein.

To verify the purification, the samples from FT, WI, WII, beads and purified protein were analyzed by SDS-PAGE and western blot. 


\subsection{Generation of RNF157 transgenic mouse}

The RNF157 knockout ES cells generated by Texas A\&M Institute for Genomic Medicine (TIGM, USA) were from a C57/BL6 mouse and injected into a blastocyst from a FVB/N mouse. In these ES cells, a gene trap cassette containing a $\beta$-galactosidase reporter gene and a neomycin resistance gene was inserted into the second intron of the RNF157 gene, so that the fusion mRNA only encodes the first 2 exons and thus the transcription of RNF157 is disrupted. The chimeric animals were crossed with wild type C57/BL6 mice to achieve a germline transmission.

\subsubsection{Isolation of genomic DNA}

Tail tips from RNF157 transgenic mice were incubated with tail lysis buffer ( $200 \mu 1$ / tail tip) for 3 hours or overnight at $55^{\circ} \mathrm{C}$. After flicking the samples to resuspend the DNA, the samples were spun down at $13000 \mathrm{rpm}$ for $5 \mathrm{~min}$ at RT. The supernatant was transferred to fresh tubes and $500 \mu \mathrm{l}$ of $100 \% \mathrm{EtOH}$ was added to precipitate the DNA. Following centrifugation at 13000 rpm for $10 \mathrm{~min}$, the supernatant was discarded, the precipitated DNA was washed with $70 \%$ EtOH and resuspended in 50-100 $\mu$ l of $\mathrm{H}_{2} \mathrm{O}$.

\subsubsection{Genotyping of RNF157 mice}

Two PCR reactions were set up for each sample, one using wild-type primer pairs (primer 28743/28744) and the other using mutant pairs (primer 25171/25172). The PCR reactions were set up with the GoTaq ${ }^{\circledR}$ DNA polymerase as following:

PCR reaction setup

\begin{tabular}{|c|c|}
\hline $0.5 \mu 1$ & genomic DNA (10 ng/ $\mu \mathrm{l})$ \\
\hline $1 \mu 1$ & forward primer $(10 \mathrm{pmol} / \mu \mathrm{l})$ \\
\hline $1 \mu 1$ & reverse primer $(10 \mathrm{pmol} / \mu \mathrm{l})$ \\
\hline $0.4 \mu 1$ & dNTPs (2.5 mM) \\
\hline $0.1 \mu 1$ & GoTaq $^{\circledR}$ DNA polymerase \\
\hline $5 \mu 1$ & $5 \mathrm{X}$ GoTaq ${ }^{\circledR}$ buffer \\
\hline $17 \mu 1$ & $\mathrm{H}_{2} \mathrm{O}$ \\
\hline
\end{tabular}


The following program was for the PCR:

RNF157 genotyping PCR

\begin{tabular}{l|l|l|l}
\hline Denaturation & $95^{\circ} \mathrm{C}$ & $3 \mathrm{~min}$ & \\
\hline Denaturation & $95^{\circ} \mathrm{C}$ & $30 \mathrm{sec}$ & \\
\cline { 1 - 3 } Annealing & $48^{\circ} \mathrm{C}$ & $30 \mathrm{sec}$ & \multirow{2}{*}{30 cycles } \\
\hline Elongation & $72^{\circ} \mathrm{C}$ & $25 \mathrm{sec}$ & \\
\hline Final Elongation & $72^{\circ} \mathrm{C}$ & $5 \mathrm{~min}$ &
\end{tabular}

\subsection{Behavioral analyses}

After weaning and during the period of behavioral testing, mice were housed in groups of 4 or 5 in standard plastic cages $(26.5 \times 20 \times 14 \mathrm{~cm})$ under a standard 12-hour light/dark cycle with food and water ad libitum, unless mentioned elsewhere. The temperature in the behavioral facility was kept at $21 \pm 2{ }^{\circ} \mathrm{C}$. All experiments were conducted in a blinded manner during the light phase of the day.

The experiment started when the animals were $9 \pm 1$ weeks old. All the tests were carried out in an order oriented toward increasing invasiveness as described before (Bodda et al., 2013): elevated plus maze for examining anxiety, open field for spontaneous activity, hole board for exploratory behavior, grip strength and rotarod for motor force, balance and coordination, marble burying for stereotypic, obsessive-compulsive behaviors, visual cliff for vision, the startle response to randomly presented acoustic stimuli ranging from $65 \mathrm{~dB}$ to $120 \mathrm{~dB}$ for hearing, prepulse inhibition of the startle response for sensorimotor gating, social interaction for sociability, buried food finding for olfaction, hot plate test for sensitivity of pain, fear conditioning for associative memory and Morris water maze for spatial learning and working memory.

\subsubsection{Elevated plus-maze}

To explore anxiety in mice, the elevated plus-maze was used. The maze was made of gray Perspex with a $5 \times 5 \mathrm{~cm}$ central platform and 2 open, 2 enclosed $30 \times 5 \times 15 \mathrm{~cm}$ arms. Individual mice were placed on the central platform, facing an open arm of the plus-maze inside a room with light intensity of 120 lx. Exploratory behavior was monitored by an overhead video camera 
connected to a PC with "Viewer 2" software (Biobserve GmbH, Bonn, Germany) to record the time each animal spent in open or closed arms. The proportion of time spent in open arms was used as readout of anxiety or anxiolytic-like behavior.

\subsubsection{Open field}

Locomotor activity was assessed using the open field test. In this test, a grey Perspex arena (120 $\mathrm{cm}$ in diameter, $25 \mathrm{~cm}$ high) was placed inside a room with light intensity of $120 \mathrm{~lx}$. The mouse was released from the center of the arena and allowed to explore the open field for $7 \mathrm{~min}$. The behavior was recorded by video camera linked to a PC equipped with "Viewer 2" software. The velocity, distance traveled and time spent in central, intermediate or peripheral zones of the open field were recorded.

\subsubsection{Hole board}

The hole board test was used to assess exploratory activity. The apparatus comprised a 50 $\mathrm{cm} \times 50 \mathrm{~cm} \times 36 \mathrm{~cm}$ transparent Perspex chamber with a non-transparent floor raised $3 \mathrm{~cm}$ above the bottom of the chamber with 16 holes of $2.2 \mathrm{~cm}$ diameter in a grid-pattern. The test was applied in a room with low illumination (5 1x). Individual mice were placed into the center and allowed to explore the chamber for $5 \mathrm{~min}$. The number of explored holes (nose pokes) was monitored by 2 layers of infrared photo beams connected to a computer with AKS software (TSE Systems GmbH, Bad Homburg, Germany).

\subsubsection{Pre-pulse inhibition}

In order to test the sensorimotor gating, the mice were placed individually in small metal cages $(90 \times 40 \times 40 \mathrm{~mm})$ to restrict major movements and exploratory behavior. The cages were equipped with a movable platform attached to a sensor that records vertical movements of the platform. The individual cages were placed into sound-attenuating isolation cabinets (TSE Systems $\mathrm{GmbH}$ ). Startle reflexes were evoked by acoustic stimuli delivered from a loudspeaker that was suspended above the cage and connected to an acoustic generator. The startle reaction to an acoustic stimulus evokes a movement of the platform. The transient force resulting from this 
movement of the platform was recorded with a computer during a recording window of $260 \mathrm{~ms}$ (beginning with the onset of pre-pulse) and saved for further evaluation. From the onset of the acoustic stimulus, the mice's reaction was recorded. Each experimental session started with a 2 min habituation to $65 \mathrm{~dB}$ background white noise, which was presented throughout the session, and followed by a 1-min baseline recording. Next, 6 pulse-alone trials with startle stimuli of 120 $\mathrm{dB}$ intensity and $40 \mathrm{~ms}$ duration were played in order to decrease influence of within-session habituation. These measurements were not included in the analysis. To assess pre-pulse inhibition, the $120 \mathrm{~dB} / 40 \mathrm{~ms}$ startle pulse was applied either alone or preceded by $20 \mathrm{~ms} 70 \mathrm{~dB}$, $75 \mathrm{~dB}$, or $80 \mathrm{~dB}$ pre-pulses. Between each pre-pulse and pulse stimulus, a $100 \mathrm{~ms}$ background white noise was presented. All trials were presented in a pseudorandom order with inter-trialintervals varying from 8 to $22 \mathrm{sec}$. The amplitude of the startle response (in arbitrary units) was defined as the difference between the maximum reaction detected during a recording window and the reaction measured right before the stimulus started. Amplitudes for stimulus alone trials and stimulus preceded by a pre-pulse were averaged for each mouse separately. Pre-pulse inhibition was calculated as the percentage of the startle response as below:

$$
\% \text { pre-pulse inhibition }=100-\frac{\text { startle amplitude after pre-pulse and pulse }}{\text { startle amplitude after pulse only }} \times 100
$$

\subsubsection{Rotarod test}

Rotarod is a test for motor coordination. In this test, a rotarod apparatus consisting of a rotating drum, automatic timers and falling sensors was used (MED Associates Inc., Vermont, USA). During test, the drum is accelerated from 4 to $40 \mathrm{rpm}$ over the course of $5 \mathrm{~min}$. After each mouse was placed on the drum, acceleration was started. The latency a mouse stayed on the drum till falling was recorded. Motor learning was measured by repeating the test 24 hours later.

\subsubsection{Grip Strength}

To assess the forelimb grip strength, a grip strength meter (TSE Systems GmbH) was used. Mice were held by their tail and lifted to enable then grasp a wire grid using their forepaws. The mice were then gently pulled backward facing the surface of the table until they released the grid. The peak force applied by the forelimbs of the mouse was recorded. Each mouse was tested three times, and the average of these three measurements was used for further statistical analysis. 


\subsubsection{Hearing}

In order to restrict mouse movement, individual mice were placed into small metal cages (90 $\times 40 \times 40 \mathrm{~mm}$ ), which were attached with a movable platform connected to a sensor that records vertical movements of the mice. Then, the cages were placed in sound-attenuating cabinets individually (TSE Systems GmbH). Mice were exposed to acoustic stimuli delivered from a loudspeaker, which were controlled by an acoustic generator. The startle response evoked by an acoustic stimulus was recorded with a computer for further analysis. The recording period was defined from the onset of the acoustic stimulus. Each session started with a 2 min habituation to $65 \mathrm{~dB}$ background white noise that lasted throughout the session, which was followed by a baseline recording for $1 \mathrm{~min}$. Afterwards, stimuli with different intensity were presented and each stimulus lasted for $40 \mathrm{~ms}$. The intensity of stimuli ranged from $65 \mathrm{~dB}$ to $120 \mathrm{~dB}$, increased with $3 \mathrm{~dB}$ per step. All intensity was presented 10 times in a pseudorandom manner with an inter-stimulus-interval of 8 to $22 \mathrm{sec}$. The amplitude of the startle response (in arbitrary units) to each stimulus was defined as the difference between the maximum movement detected and the movement measured immediately before the stimulus onset. Amplitudes of responses toward each stimulus with same intensity were averaged for individual mice.

\subsubsection{Marble burying}

This test was applied to investigate stereotypes and obsessive-compulsive behavior in mice. A plastic cage $(34.5 \times 56.5 \times 18 \mathrm{~cm})$ filled with $5 \mathrm{~cm}$ deep wood-chip bedding was placed in a room with dim light (6 lx). 24 glass marbles were positioned on top of the bedding. The marbles were placed in 6 rows with 4 marbles per row at a distance of $4 \mathrm{~cm}$. Each mouse was released into the cage and could freely manipulate the marbles for $30 \mathrm{~min}$. Number of buried marbles (to $2 / 3$ their depth) was counted.

\subsubsection{Visual cliff}

To assess more profound visual deficiencies, e.g. contrast perception, a transparent Perspex board $(63 \times 29 \mathrm{~cm})$ attached to a grey Perspex shelf $(31.5 \times 29 \times 54 \mathrm{~cm})$ as depicted below was used: 


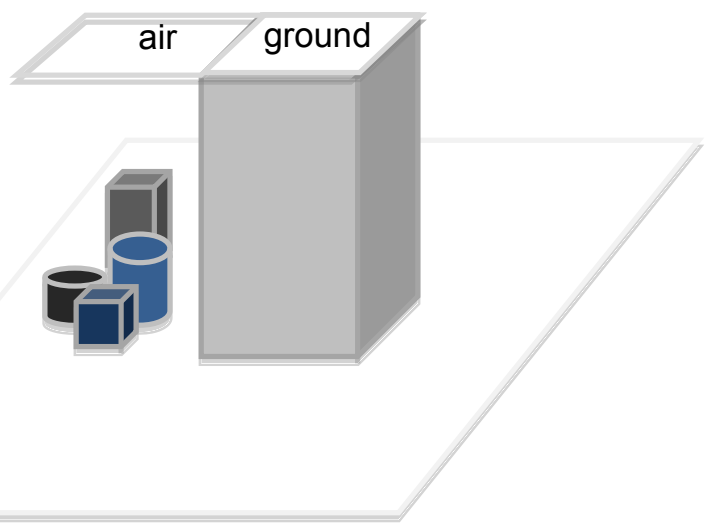

The transparent board was positioned in the way that half of the board was placed on the shelf with white paper below ("ground" side), and the other half was $54 \mathrm{~cm}$ above the floor ("air" side). Some objects were placed around the apparatus as visual cues for depth perception. Mice were placed in the middle of the "ground" area, and their activity was monitored for 5 min by a computer and the video-tracking system "Viewer 2" (Biobserve $\mathrm{GmbH}$,). The time spent in each area was recorded and the percentage of time in the "ground" and the "air" areas was used for statistical analysis. The light intensity in the room was $120 \mathrm{~lx}$.

\subsubsection{Novel object recognition}

This test was designed to assess an animal's spatial and non-spatial working memory, following the modified protocol as described previously (Roullet et al., 1996). The apparatus used was a grey Perspex arena $(60 \mathrm{~cm}$ in diameter, $40 \mathrm{~cm}$ high) positioned in the middle of a room with illumination at $120 \mathrm{~lx}$. The ground of the arena was divided into different sectors and covered by a layer of glass as illustrated below. As the only visual cue in the uniformed environment, a striped pattern $(20 \times 10 \mathrm{~cm})$ was attached to the wall of the apparatus.

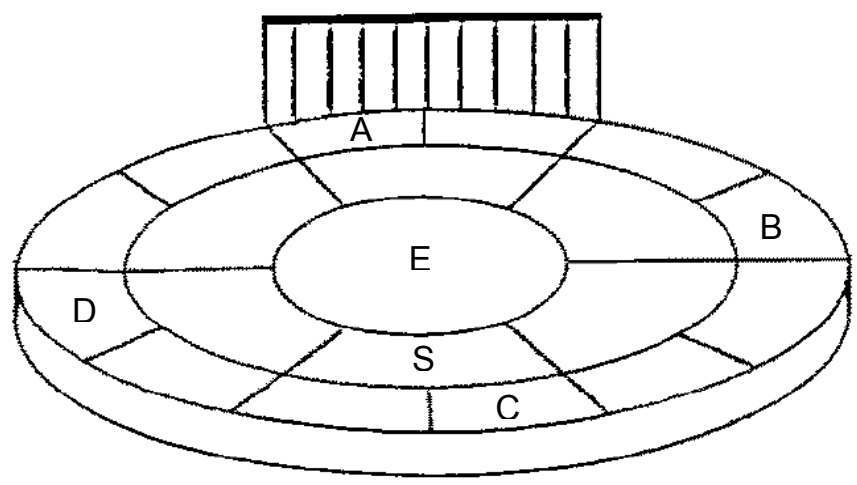

(Adapted from Roullet et al., 1996) 
Every mouse underwent 7 sessions. After released from position "S", animals were allowed to explore the area for $7 \mathrm{~min}$ with the 3 min inter-session interval. In the session 1, individual mice were placed into the empty arena for habituation. From session 2 to 4 , four different white Perspex objects were placed in a square arrangement at position A, B, C and D. During session 5 and 6, the object at position D was moved to the position E for assessing the spatial memory. In the final session 7, the white object at position B was replaced by a new black object for examining the non-spatial memory. The activity and time mice spent on exploring individual objects were recorded by a computer and the video-tracking system "Viewer 3" (Biobserve $\mathrm{GmbH})$.

\subsubsection{Olfaction}

Two days before habituation, 2 pieces of chocolate cookies per animal per day were placed into the cages. For habituation to testing environment, mice were placed into the test cages (29.5 $\times 18.5 \times 13 \mathrm{~cm}$ ) with a visible piece of cookie inside per trial. The cookie was placed randomly at the positions $\mathrm{A}, \mathrm{B}, \mathrm{C}$ or $\mathrm{D}$ as illustrated:

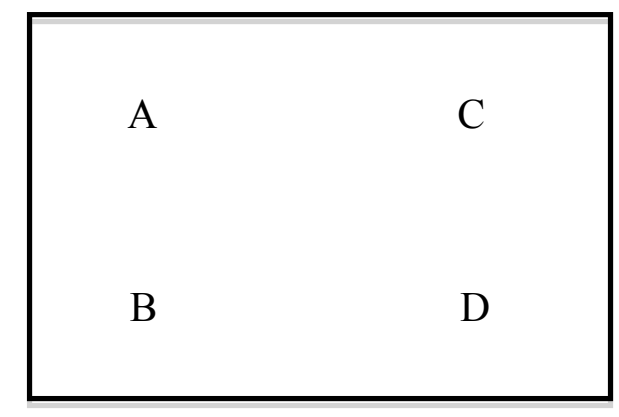

In every trial, mice were released in the center of the cage and allowed to explore the environment for $10 \mathrm{~min}$. All mice underwent 4 trials per day with an inter-trial interval of approx. $50 \mathrm{~min}$. After two days of habituation, mice were food deprived overnight while water was available ad libitum and 2 small pieces of cookie were placed within the homecage.

On day 5 the test took place. Individual mice were placed into test cages with a chocolate cookie hidden $1 \mathrm{~cm}$ under the standard bedding at position A, B, C or D. Mice were allowed to look for the hidden cookie for 5 min per trial. Each animal was tested twice with an inter-trial interval of approx. $100 \mathrm{~min}$. The latency from the time when the animals were released in the cage until they found the cookie was recorded. The average time of two trials was the measure of 
the integrity of the olfactory system.

In order to control for other sensory, motor or motivational deficiencies that might influence the food-finding latency, the test was repeated as described above with a piece of cookie clearly visibly placed on the bedding. A fresh cage and bedding was used for each trial, and all mice were subjected to identical testing procedures.

\subsubsection{Morris water maze}

Spatial learning and memory was assessed in a water maze as described previously (Morris, 1984; Radyushkin et al., 2010). Firstly, a circular tank (diameter $1.2 \mathrm{~m}$, depth $0.4 \mathrm{~m}$ ) was filled with opaque water $\left(25 \pm 1^{\circ} \mathrm{C}\right.$, depth $\left.0.3 \mathrm{~m}\right)$. The swim patterns of mice were monitored by the video-tracking system "Viewer 3" (Biobserve $\mathrm{GmbH}$ ). The escape latency, swim speed, path length and trajectory of swimming were recorded for each mouse. During the first 2 days, mice were trained to find a hidden escape platform $(10 \times 10 \mathrm{~cm})$ submerged $1 \mathrm{~cm}$ below the water surface tagged with a $15-\mathrm{cm}$ high black flag. The platform was located pseudo-randomly in different locations across trials. Mice underwent two days of training in the visible condition, followed by hidden platform training (acquisition) where the flag was removed. For 8 days, mice were trained to find a hidden platform that was placed at the center of one of the four quadrants of the tank. Throughout the acquisition, the platform for each mouse was fixed. Mice had to orientate themselves using spatial cues that were placed on the walls of the testing room. Every day, each mouse performed four trials with an inter-trial interval of $5 \mathrm{~min}$. Mice were released into the pool facing the sidewall at one of four start locations and allowed to search for the platform until they found it, or for a maximum of $90 \mathrm{sec}$. Any mouse that failed to find the platform within $90 \mathrm{sec}$ was guided to the platform and remained on the platform for $20 \mathrm{sec}$ before being removed from the pool. The next day after the hidden platform training, a probe trial was conducted to determine if mice developed a spatial preference to the location where the platform was. After removal of the platform, mice were allowed to swim freely for $90 \mathrm{sec}$. The percentage of time spent in each quadrant of the pool as well as the number of times the mice crossed the former position of the hidden platform were recorded. When no genotype differences were observed, mice were subjected to the reversal spatial learning after the probe trial. The reversal learning was applied following the same procedure as the spatial training but the platform was placed on the opposite quadrant of the previous location used in the hidden platform acquisition. The reversal learning lasted for four days followed by a probe trial. 


\subsubsection{Hot plate test}

To measure pain perception, the hot plate test was applied. Mice were placed onto a metal plate (Ugo Basile, Italy) that was preheated up to $55^{\circ} \mathrm{C}$. The latency to show hind paw licking or jumping was recorded. Mice were removed from the plate immediately after appearance of the response or after $40 \mathrm{sec}$ stayed on the plate to protect mice from injury.

\subsubsection{Cued and contextual fear conditioning}

The fear conditioning test was applied to assess the mice's associative memory. Mice were first trained within the same session for both contextual and cued fear conditioning. During the training session, mice were placed in the conditioning chamber (context) for $120 \mathrm{sec}$ to assess the baseline level of activity. This period was followed by a $10 \mathrm{sec}, 5 \mathrm{kHz}, 85 \mathrm{~dB}$ tone (conditioned stimulus, CS). Immediately after the tone, a $2 \mathrm{sec}, 0.4 \mathrm{~mA}$ foot shock (unconditioned stimulus, US) was applied. This CS-US pairing was repeated $13 \mathrm{sec}$ later. The individual mice remained in the chamber for an additional $23 \mathrm{sec}$ following the second CS-US pairing. 24 hours after training, mice were positioned into the same context (the same chamber) and monitored over 2 min for freezing to examine the contextual memory. The cued memory test was then performed 27 hours after training in a new chamber. First, freezing response in the new context was monitored over a $2 \mathrm{~min}$ pre-cue period with no tone followed by a $2 \mathrm{~min}$ cued period when the tone (cue) was presented. Duration of freezing behavior, defined as the absolute lack of movement (excluding respiratory movements), was recorded by a video camera connected to a PC equipped with "Video Freeze" software (MED Associates Inc.). 


\section{Results}

\subsection{Characterization of the expression and function of RNF157}

The balance between cell survival and cell death is essential for the development and maintenance of a functional neuronal network. Therefore, the molecules that mediate neuronal apoptosis draw much attention. Accumulating evidence suggests a role of the ubiquitinproteasome system (UPS) in controlling apoptosis. Since E3 ligases function as quality control in UPS, we attempted to investigate the role of E3 ligases in neuronal apoptosis. Here, the braindominant E3 ligase RNF157 was in focus. Our previous gain- and loss-of-function analyses indicated that the brain-dominant E3 ligase RNF157 promotes survival in cerebellar granule neurons. Subsequent experiments showed that Fe65 acts downstream in the RNF157-mediated survival pathway (Matz et al., in press). Following these results, I used both in vitro and in vivo techniques to expand the understanding of RNF157 as a regulator of neuronal survival.

\subsubsection{RNF157 is present in neuronal and non-neuronal cell types in the brain}

Previous analyses in our lab showed the E3 ligase RNF157 is predominately expressed in the postnatal and adult brain, with abundant expression in e.g. cerebellar granule neurons and cortical neurons (Matz et al., in press). Here, I carried out further analyses of RNF157 expression in other cell types.

Started out by validating the specificity of a new RNF157 antibody, I generated a recombinant rat RNF157 protein (Fig 3.1a) that contains amino acids 562 to 617 of the RNF157 protein. Importantly, this recombinant protein was anticipated to be recognized by the RNF157 antibody. Next, I used lysates of cultured cortical, hippocampal and cerebellar granule neurons and subjected them to immunoblotting with RNF157 antibody. While I found a band at $110 \mathrm{kDa}$ in the RNF157 antibody condition, the preabsorption with recombinant RNF157 protein led to the disappearance of this band (Fig 3.1b), indicating the specificity of the RNF157 antibody. It is also noteworthy to mention that the calculated molecular weight of RNF157 (70 kDa, 644 amino acids) differed from the $110 \mathrm{kDa}$ owing to the acidic nature of RNF157, which results in slow migratory behavior (Matagne et al., 1991). Furthermore, lysates from astrocytes and oligodendrocyte precursor cells (OPC) were included for immunoblotting analysis with RNF157 
antibody (Fig 3.1c). Interestingly, RNF157 could also be detected in these non-neuronal cell types. Taken together, RNF157 is expressed in astrocytes and oligodendrocytes in addition to its robust expression in cultured cortical, hippocampal and cerebellar granule neurons.

a

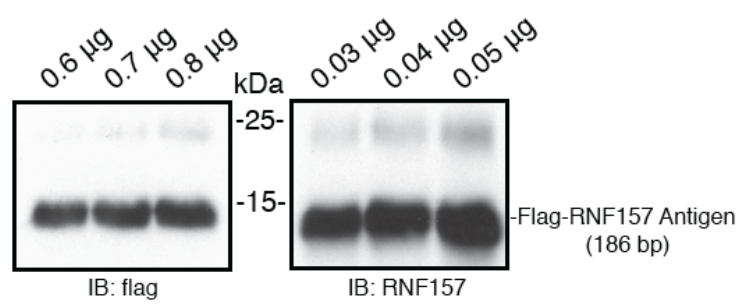

b

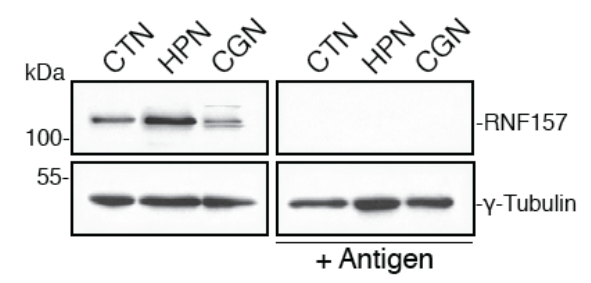

C

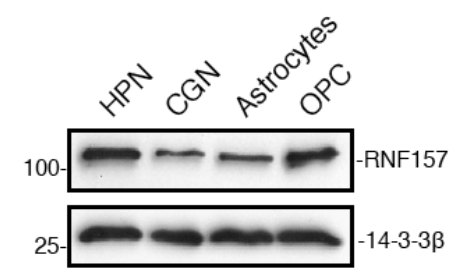

Fig 3.1 RNF157 is expressed in different cell types in the brain.

a) Immunoblotting of recombinant RNF157 protein using Flag or RNF157 antibodies.

b) Cultured cortical (CTN), hippocampal (HTN) and cerebellar granule neurons (CGN) were lyzed and subjected to immunoblotting with RNF157 antibody, RNF157 antibody preabsorbed with antigen or $\gamma$-Tubulin antibody. $\gamma$-Tubulin served as loading control.

c) Lysates from cultured hippocampal (HTN), cerebellar granule neurons (CGN), astrocytes and oligodendrocyte precursor cells (OPC) were immunoblotted with RNF157 and 14-3-3 $\beta$ antibodies. The latter served as loading control.

\subsubsection{RNF157 is strongly expressed in various brain regions}

Following the expression analysis in cell lysates, I went on to examine the spatial distribution of RNF157. In order to analyze the expression pattern and function of RNF157 in vivo, we generated a conventional mouse, in which a gene trap containing $\beta$-Geo gene was inserted into the second intron of Rnf157 gene to disrupt the transcription of RNF157 (Fig 3.2a). Instead, the $\beta$-Geo gene results in the expression of $\beta$-galactosidase driven by the endogenous RNF157 promoter. For genotyping, two sets of primers were designed to specifically detect the wild type or the mutant allele as described in chapter 2.12.2. The result of a representative genotyping PCR is illustrated in Fig 3.2b.

Next, I verified if the gene trap successfully deleted the expression of full length RNF157. Lysates of cortices and hippocampi isolated from wild type, heterozygous and homozygous postnatal day 5 (P5) RNF157 transgenic mice were subjected to immunoblotting with the $\beta$-Gal antibody and the RNF157 antibody. $\beta$-Gal was expressed in the RNF157 $7^{+/-}$and the amount increased in RNF157/- brain tissue. Furthermore, complete deletion of RNF157 could be 
observed in the RNF157/- brain while only half of the amount was expressed in $\mathrm{RNF} 157^{+/-}$mice as compared to the wild type $\left(\mathrm{RNF} 157^{+/+}\right)$(Fig 3.2c). The mice with homozygous deletion of the Rnf157 gene were viable and fertile with no obvious deficits.

a

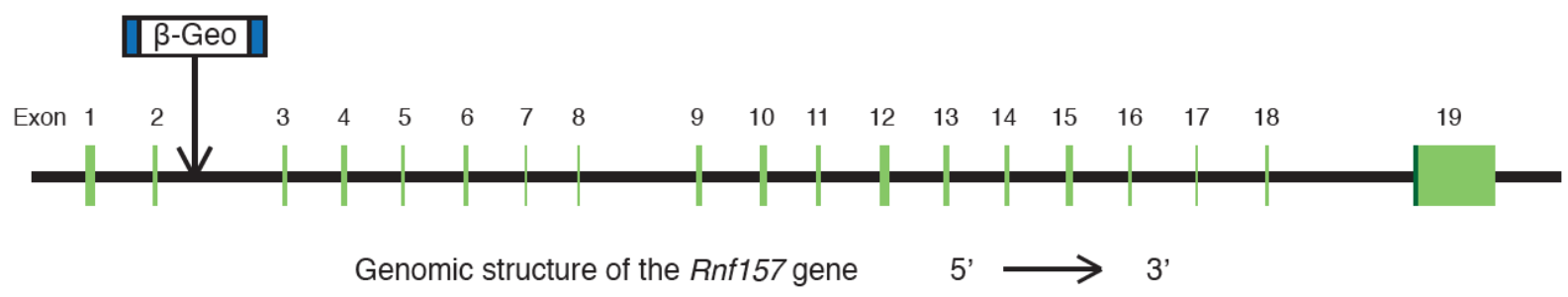

b

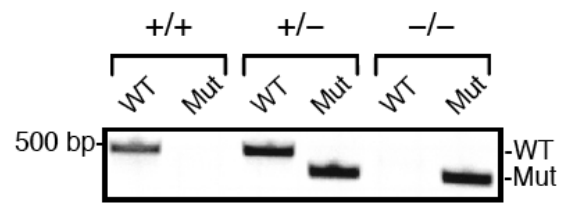

C

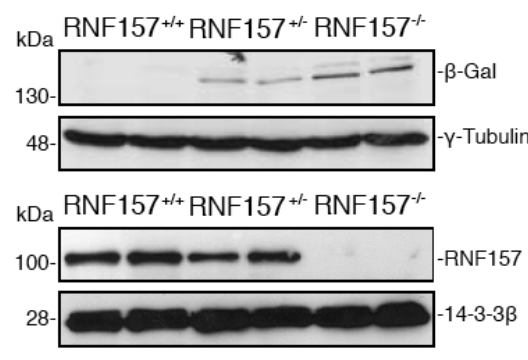

P5 Cortex
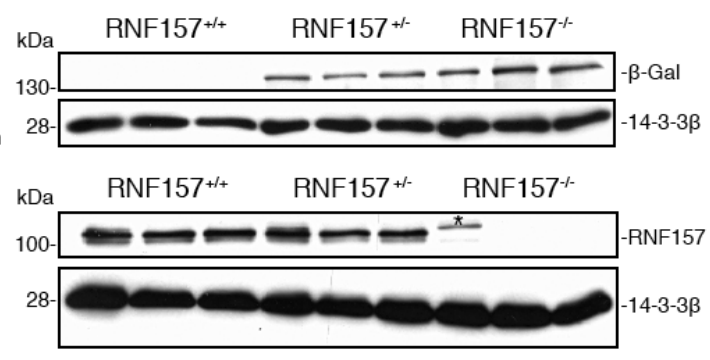

P5 Hippocampus

d

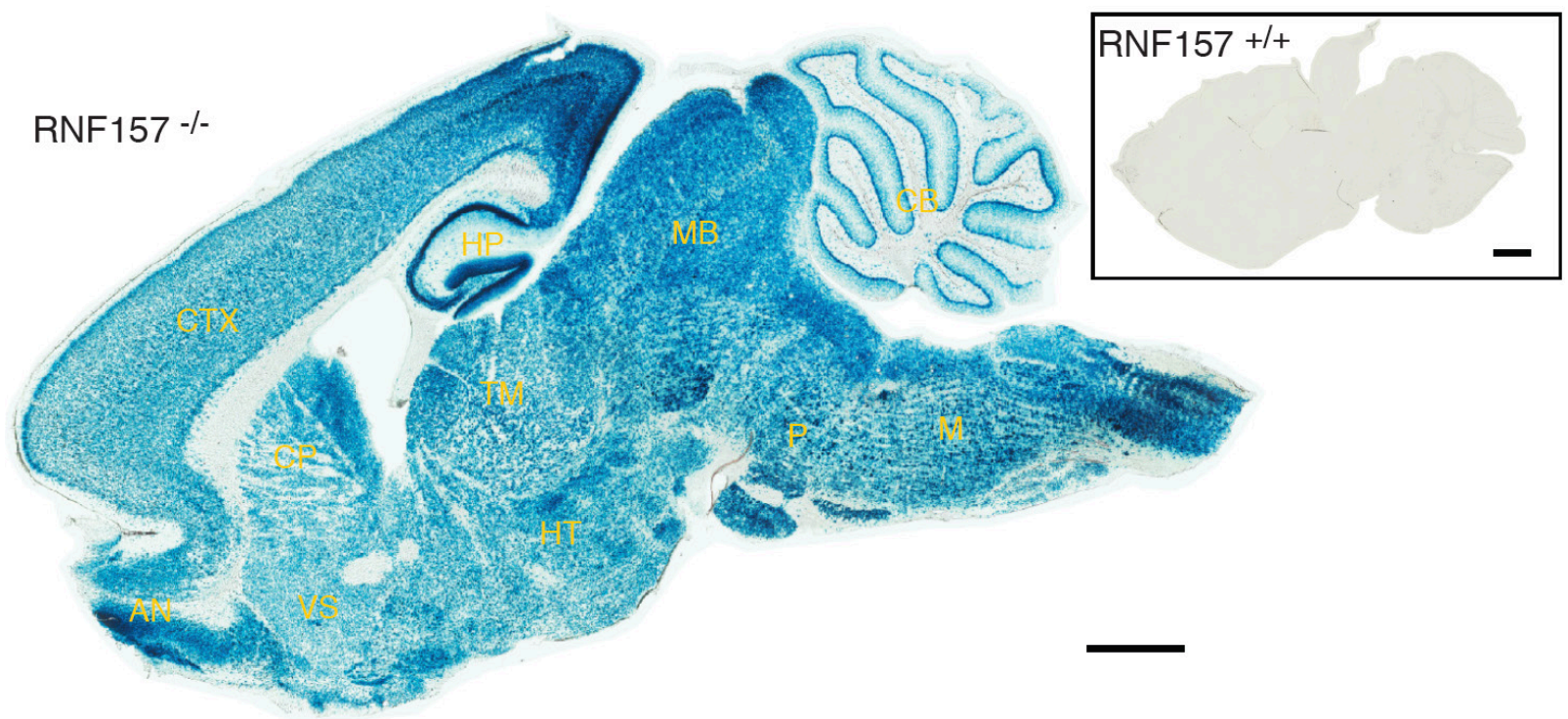


Fig 3.2 RNF157 is expressed in the brain.

a) Schematic representation of RNF157 genomic structure and the location of the gene trap cassette encoding the $\beta$-galactosidase ( $\beta$-Gal).

b) Genomic DNA was isolated from mouse tail biopsies and subjected to PCR amplification. The upper band at $498 \mathrm{bp}$ represents the wild type fragment while the lower band at $280 \mathrm{bp}$ indicates the mutant fragment.

c) Lysates of cortices and hippocampi isolated from P5 mice were immunoblotted using the $\beta$-galactosidase or the RNF157 antibody. 14-3-3 $\beta$ served as loading control. The asterisk indicates a non-specific band.

d) X-Gal staining of a sagittal brain section of a 28-day-old RNF157 homozygous mouse. Blue spots indicate the activity of RNF157 promoter. Inset is the X-Gal staining of a section from a wild type mouse, where no X-Gal signals can be detected. CTX, cortex; HP, hippocampus; MB, midbrain; CB, cerebellum; CP, caudate putamen; TM, thalamus; AN, anterior olfactory nucleus; VS, ventral striatum; HT, hypothalamus; P, pons; M, medulla. Scale bar $=1 \mathrm{~mm}$.

In addition, I subjected sagittal brain sections of P28 RNF157/- mouse and its wild type littermate to X-Gal staining (Fig 3.2d). While the staining of the section of the wild type mouse gave expectedly no results, I found strong enzymatic $\beta$-galactosidase activity throughout the whole brain of the RNF $157^{-/-}$mouse. I observed a particularly strong expression observed in the cortex, hippocampus, midbrain, thalamus and hypothalamus, while the signal in the cerebellum was more restricted to Purkinje cell layer with a low expression level in the molecular and granule cell layer. In summary, these results confirmed that indeed RNF157 is expressed in various cell types in the brain.

\subsubsection{RNF157 localizes to cytoplasm}

To refine our previous findings in which we described that RNF157 is a component of the postnuclear fraction in a subcellular fractionation experiment (Matz et al., in press), I carried out a more elaborate method to support a potential cytoplasmic localization. Together with N. Schwedhelm-Domeyer, I purified the cytoplasm from P5 wild type mouse cortex by applying two steps of ultracentrifugation at $21000 \mathrm{~g}$ and $257000 \mathrm{~g}$ to remove the plasma membrane and the heavy membrane. The collected fractions were subjected to immunoblotting analysis with the RNF157 antibody. The nuclear marker SnoN, the plasma membrane marker N-Cadherin and the cytoplasmic marker pan 14-3-3 were included as quality control for the fractions. The result revealed that RNF157 is a cytoplasmic protein (Fig 3.3). 


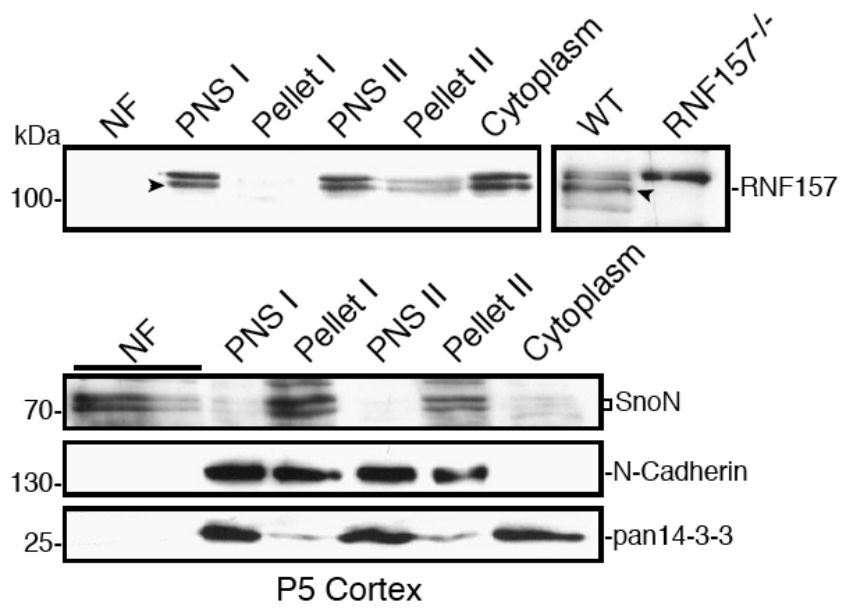

Fig 3.3 RNF157 is present in the cytoplasm.

Lysates of P5 mouse cortex was subjected to subcellular fractionation analysis. The nuclear fraction (NF) was first separated. The remaining post-nuclear supernatant (PNS) containing plasma membrane, heavy membrane and cytoplasm was further ultra-centrifuged. The resulting fractions PNS I, Pellet I, PNS II, Pellet II and cytoplasm were immunoblotted with RNF157, SnoN (nuclear fraction marker), NCadherin (plasma membrane marker) and pan 143-3 antibodies (cytoplasmic marker). Lysates of wild type and RNF157-/- mouse cortices served as positive control to indicate the specific RNF157 band.

\subsubsection{Altered major pathways regulating cell survival due to deletion of RNF157}

Having established the subcellular localization of RNF157, I determined the functional consequence of loss-of-RNF157 for neuronal survival in vivo. Although the RNF157/- mice are viable, a dysregulation of cell survival/apoptosis pathways could not be excluded. One of the critical regulators of neuronal survival is the serine-threonine kinase Akt, which has been implicated in various survival pathways and whose active phosphorylated form was reported to be neuroprotective (Datta et al., 1999; Lu and Xu, 2006b). Another important regulator of cell survival, the extracellular-signal-regulated kinase, Erk, has been shown to have pro-survival as well as pro-apoptotic functions in various conditions ( $\mathrm{Lu}$ and $\mathrm{Xu}, 2006 \mathrm{~b}$ ). Hence, I went on to investigate the level of total Akt, total Erk, activated Akt and activated Erk in the RNF157/- mice.

P5 cortical and hippocampal tissues from RNF157//- animals and their control littermates were subjected to immunoblotting with Erk, phosphorylated Erk (pErk), Akt and phosphorylated Akt (pAkt) antibodies. I found that the total amount and the active forms of these kinases were unchanged at P5 (Fig 3.4a, b). Next, cerebella, hippocampi and cortices of 10-week-old mice were lysed and immunoblotted with the abovementioned antibodies. Surprisingly, while the total amount of Akt and Erk remained unaltered in the 10-week-old mice, a clear reduction of pAkt and pErk was detected (Fig 3.4c). These results indicated that although loss-of-RNF157 did not have an immediate effect upon neuronal survival in vivo, but it induced an age-dependent alteration of the major survival/apoptotic regulators. Taken together, these data support the notion that RNF157 is involved in maintaining neuronal health. 
a

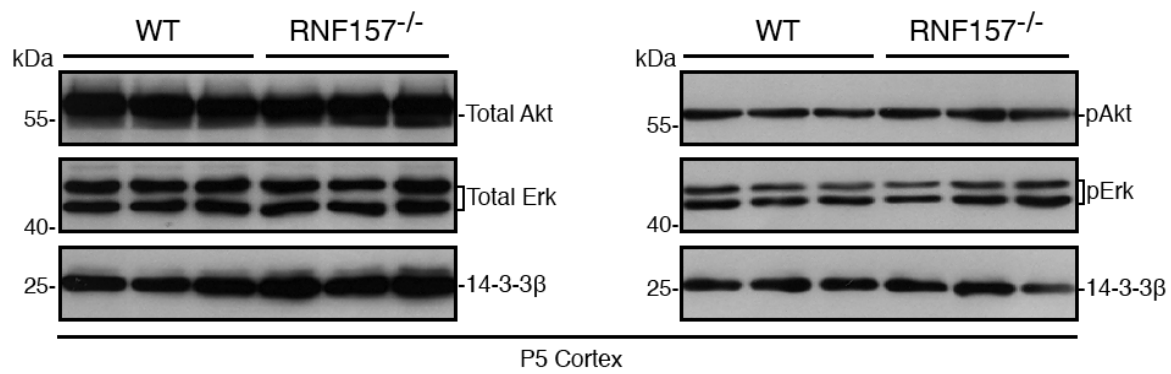

b

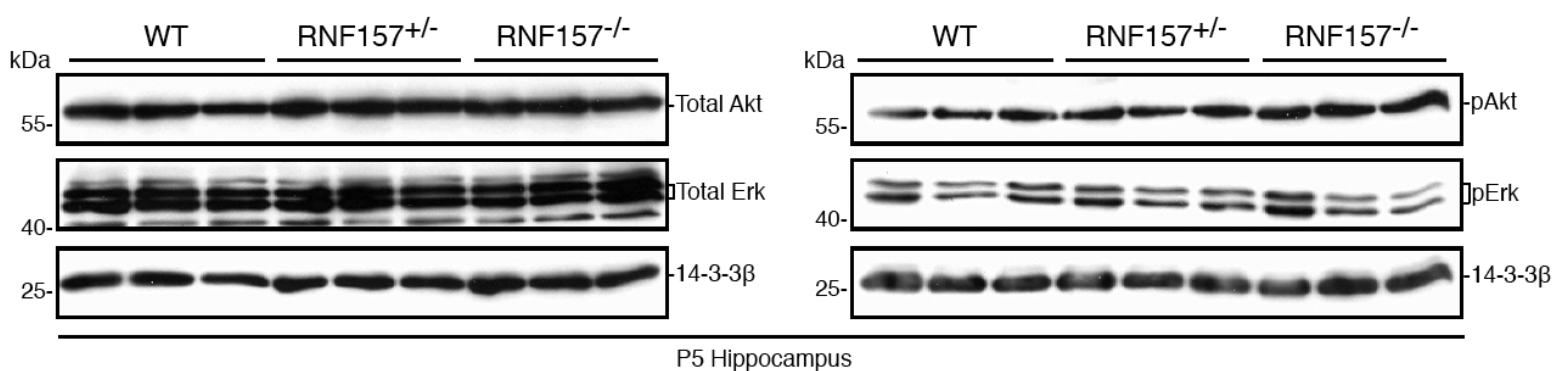

C

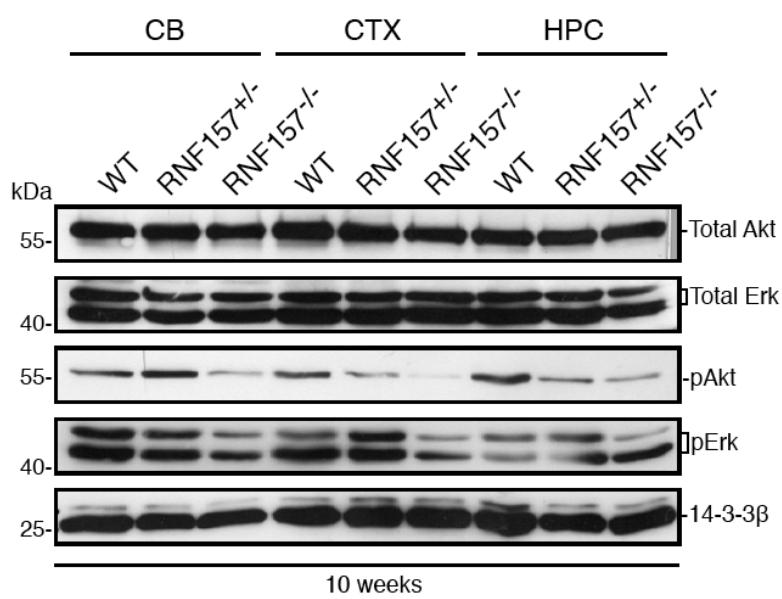

Fig 3.4 Loss of RNF157 led to agedependent downregulation of active Akt and active Erk.

a) Cortical and b) hippocampal lysates of P5 wild type, RNF157 heterozygous or homozygous mice were immunoblotted using the total Akt, total Erk, phosphorylated Akt (pAkt), phosphorylated Erk (pErk) and 14-3$3 \beta$ antibodies. The latter served as loading control.

c) Tissue lysates of 10-week-old mouse cerebella, cortices and hippocampi were subjected to immunoblot analysis with the total Akt, total Erk, phosphorylated Akt (pAkt), phosphorylated Erk (pErk) and 14-3$3 \beta$ antibodies. The latter serves as loading control. Courtesy of N. SchwedhelmDomeyer.

\subsubsection{Functional analysis of RNF157 in hippocampal neurons}

Having confirmed a potential role of RNF157 in neuronal viability in vivo, I set out to analyze the function of RNF157 in hippocampal neurons. A previous study in our lab described that RNF157 regulates neuronal survival using cerebellar granule neurons as a model system (Matz et al., in press). Since RNF157 exhibited a robust expression in hippocampal neurons, I asked if the pro-survival RNF157 pathway also operates in hippocampal neurons. To answer this 
question, I first examined the efficacy of the RNF157 RNAi construct in neurons for the further experiments.

Since RNF157 RNAi construct \#3 was most efficient in knocking down exogenous RNF157 in HEK293T (Matz et al., in press), I have used this plasmid throughout the study and referred to it as RNF157 RNAi. To examine endogenous knockdown of RNF157 in neurons, I nucleofected isolated cortical neurons with U6 control vector or with RNF157 RNAi plasmid together with the Bcl-xL and GFP expression plasmids. At day in vitro (DIV) 5, neurons were lysed and subjected to immunoblotting with RNF157 and $\gamma$-Tubulin antibodies (Fig 3.5a). The result indicates that expression of the RNF157 RNAi plasmid induces efficient endogenous knockdown of RNF157 in cortical neurons.

It has been demonstrated that loss-of-RNF157 in cerebellar granule neurons as well as hippocampal neurons leads to cell death (Matz et al., in press). To exclude that the RNF157 RNAi-induced apoptosis in hippocampal neurons is an off-target effect, I carried out rescue experiments using the RNF157-Rescue (RNF157-Res) construct previously generated in the lab. RNF157-Res harbors 6 silent mutations in the RNF157 RNAi targeting region and thus is resistant to knockdown. Cultured hippocampal neurons were transfected at DIV2 with the control vector, RNF157 RNAi, RNF157-Res plasmid, or both of the RNF157 RNAi and RNF157-Res expression plasmids together with the $\beta$-Gal construct. After 5 days, hippocampal neurons were subjected to immunocytochemistry and analyzed. Here, I found that while RNF157 knockdown led to a significant increase in cell death, expression of RNF157-Res in the background of RNF157 knockdown significantly protected hippocampal neurons from apoptosis (Fig 3.5b). Together with our previous finding, my result suggests that RNF157 serves as a prosurvival protein in hippocampal neurons.

a

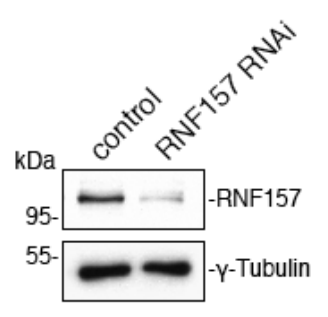

b

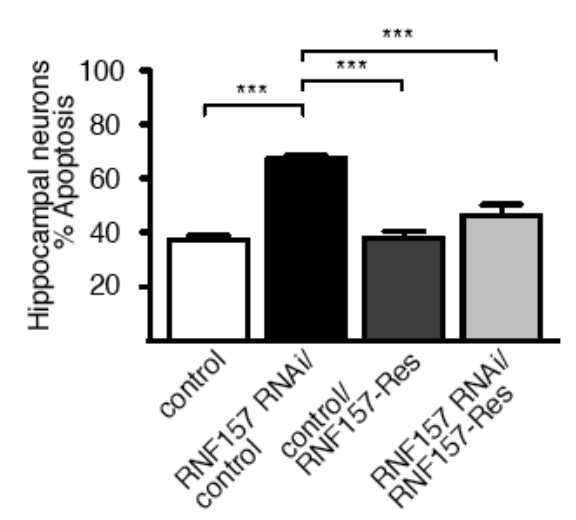


Fig 3.5 Knockdown of endogenous RNF157 leads to increased apoptosis in hippocampal neurons.

a) Lysates of cortical neurons, nucleofected with U6 control plasmid or RNF157 RNAi plasmid, were immunoblotted with RNF157 and $\gamma$-Tubulin antibodies. The latter served as loading control.

b) Hippocampal neurons transfected at DIV2 with control vector, the RNF157 RNAi, the RNF157-Res plasmid, or both the RNF157 RNAi and RNF157-Res plasmids together with $\beta$-gal expression plasmid, were fixed after 5 days and analyzed. A total of 1099 neurons from 4 independent experiments were included in the analysis (ANOVA, $* * * \mathrm{p}<0.001$, mean + s.e.m.).

\subsection{Fe65, a novel interactor of RNF157, regulates neuronal survival in different neuronal types}

Previously, Fe65 has been identified as an interactor of RNF157 and overexpression of Fe65 promotes cell death in cerebellar granule neurons (Matz et al., in press). I reasoned that Fe65 could have the same role in controlling neuronal survival in hippocampal neurons. Moreover, Fe65 was reported to be present in the nuclear and cytoplasmic extracts of PC12 cells (Minopoli et al., 2001) and previous data in the lab showed that the nuclear Fe65 (NLS-Fe65, NLS refers to nuclear localization signal) but not the cytoplasmic Fe65 (NES-Fe65, NES is nuclear export signal) contributes to the apoptotic phenotype in cerebellar granule neurons (Matz et al., in press). Therefore, I first asked if Fe65 localizes to nucleus and cytoplasm in neurons. Furthermore, I addressed if Fe65 is pro-apoptotic in the hippocampal neurons and if the localization of Fe65 is essential for its role in apoptosis.

\subsubsection{Fe65 localizes to the nucleus and cytoplasm of cerebellar granule neurons and cortical neurons}

To confirm the nuclear and cytoplasmic localization of Fe65 in neurons, cultured cerebellar granule neurons and cortical neurons were subjected to subcellular fractionation experiments and immunoblotted with the Fe65, SnoN, Sp1 or 14-3-3 $\beta$ antibodies. While SnoN and Sp1 are nuclear proteins, $14-3-3 \beta$ is found in the cytoplasm. The results revealed that in neurons, Fe65 is present in the nucleus as well as in the cytoplasm (Fig 3.6a, b).

Since the Fe65 antibody failed to work in immunocytochemistry, I overexpressed myc-Fe65 in HEK293T cells and stained the cells with myc antibody together with the DNA dye DAPI. The analysis of confocal images also supported the fractionation studies that Fe65 is localized to the nucleus as well as the cytoplasm (Fig 3.6c). 
a

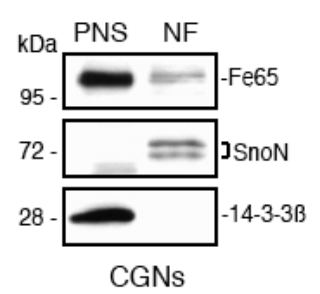

b

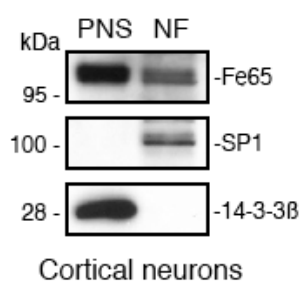

C

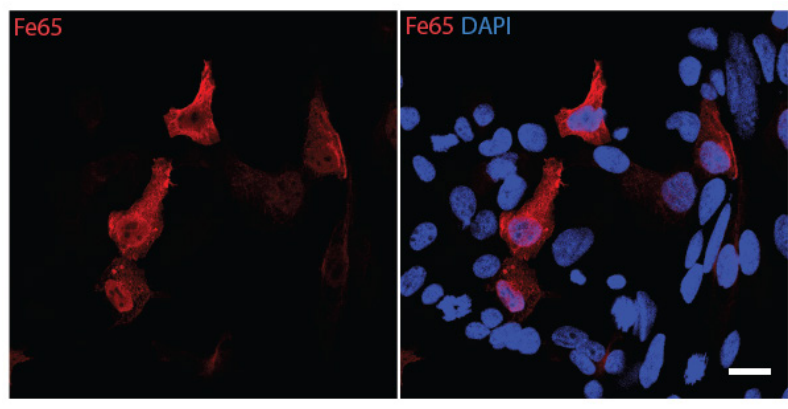

Fig 3.6 Fe65 localizes to the nucleus and cytoplasm in neurons as well as HEK293T cells.

a), b) Cerebellar granule neurons (CGNs) and cortical neurons were subjected to subcellular fractionation experiments and then immunoblotted with Fe65 antibody. SnoN and SP1 antibodies were used as quality control for postnuclear supernatant (PNS) while 14-3-3 $\beta$ served as control for nuclear fraction (NF). The analysis of cerebellar granule neurons was done by M. Kannan.

c) HEK293T cells were transfected with myc-Fe65 and subjected to immunochemistry analysis with the myc antibody and the DNA dye DAPI. Images were taken using confocal microscope. Scale bar $=20 \mu \mathrm{m}$.

\subsubsection{Nuclear Fe65 is the main contributor to cell apoptosis in hippocampal neurons}

Next, I analyzed the role of Fe65 and the relevance of its localization in neuronal survival in hippocampal neurons. I transfected cultured hippocampal neurons at DIV2 with control vector, wild type Fe65, NES-Fe65 or NLS-Fe65 plasmids together with the $\beta$-Gal plasmid. At DIV 6 , neurons were fixed and subjected to viability analysis. As previously reported by A. Matz, wild type Fe65 and NLS-Fe65 were equally potent in triggering neuronal apoptosis. Interestingly, I found that overexpression of NES-Fe65 also led to a significant increase in apoptosis. The stimulation however was not as potent as wild type Fe65 or NLS-Fe65 (Fig. 3.7). This result suggests that Fe65 operates mainly in the nucleus to mediate neuronal apoptosis.

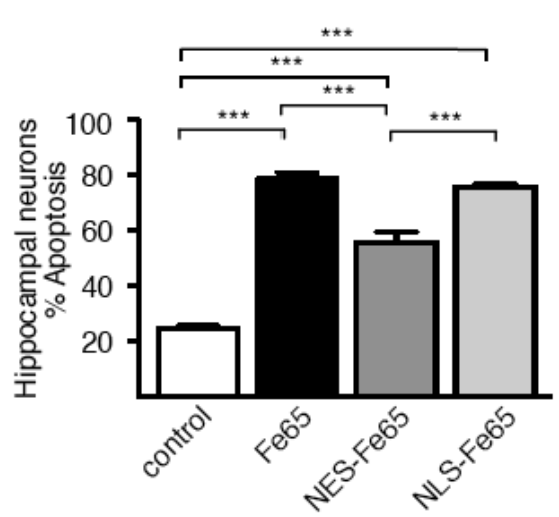

Fig 3.7 Nuclear Fe65 is the main contributor in triggering neuronal apoptosis in hippocampal neurons.

Hippocampal neurons transfected at DIV2 with control vector, wild type Fe65, NES-Fe65 or NLS-Fe65 plasmids together with $\beta$-Gal expression plasmid were fixed at DIV6 and subjected to quantification of apoptotic neurons. A total of 1361 neurons from 4 independent experiments was included in the analysis (ANOVA, $* * * \mathrm{p}<0.001$, mean + s.e.m.). 


\subsubsection{Fe65 acts downstream of RNF157 to regulate neuronal survival}

Since Fe65 is an interactor of RNF157, we then asked if they act in a linear pathway to control neuronal survival. Prior to epistasis analysis, I first verified the endogenous knockdown of Fe65 by the Fe65 RNAi plasmid. I nucleofected isolated cortical neurons with either U6 control vector or Fe65 RNAi together with the Bcl-xL and GFP expression plasmids. Neurons were lysed 5 days later and subjected to immunoblotting with Fe65 and $\gamma$-Tubulin antibodies (Fig 3.8a). The result showed that the Fe65 RNAi triggered efficient endogenous knockdown of Fe65 in cortical neurons.

a

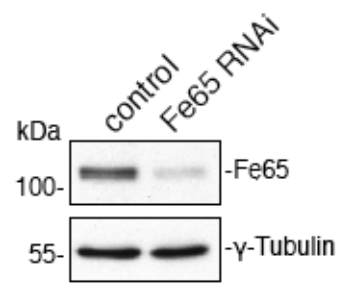

B

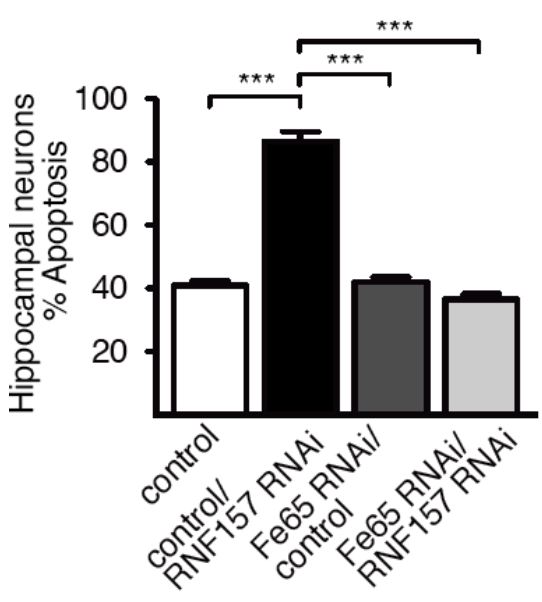

C

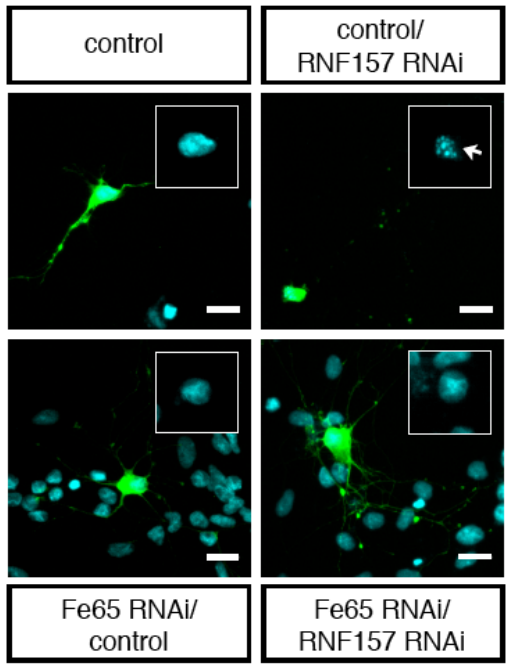

Fig 3.8 Fe65 acts downstream of RNF157 in a linear pathway to regulate neuronal survival.

a) Lysates from cortical neurons electroporated with U6 control vector or Fe65 RNAi plasmid were immunoblotted with Fe65 and $\gamma$-Tubulin antibodies. The latter served as loading control.

b) Hippocampal neurons were transfected at DIV2 with plasmids encoding control vectors, control vector with RNF157 RNAi, Fe65 RNAi with control vector, or Fe65 RNAi and RNF157 RNAi together with $\beta$-Gal plasmid. Four days later, neurons were subjected to immunocytochemistry with the $\beta$-Gal antibody and the DNA dye DAPI and the apoptotic rate was quantified. A total of 1305 neurons in 3 independent experiments was analyzed. This experiment was done by A. Matz. (ANOVA, *** $\mathrm{p}<0.001$, mean+s.e.m.).

c) Representative images of neurons in Fig 3.7b. Insets depict the nuclei of transfected neurons. The arrow indicates the pyknotic nucleus. Scale bar $=20 \mu \mathrm{m}$.

Using the Fe65 RNAi, epistasis analysis was performed by A. Matz. Here, hippocampal neurons were transfected with control vectors, RNF157 RNAi, Fe65 RNAi or both RNF157 RNAi and Fe65 RNAi together with $\beta$-Gal expression plasmid at DIV2. After 4 days, neurons were fixed and the apoptotic rate was analyzed. The neurons transfected with RNF157 RNAi showed a strong increase in apoptosis as compared to the control condition, the Fe65 knockdown or the RNF157/Fe65 double knockdown (Fig 3.8b, c). This result suggested that Fe65 
knockdown protects neurons from RNF157-knockdown induced neuronal death and indicated that Fe65 acts downstream of RNF157 in the control of neuronal survival.

\subsubsection{RNF157 ubiquitinates Fe65 with lysine 63-linked ubiquitin chain}

Having established that RNF157 acts together with Fe65 in a linear pathway in the control of neuronal survival, we set out to examine if Fe65 is a substrate of RNF157. First we analyzed if RNF157 has ligase activity and could autoubiquitinate itself. Since the RING domain is necessary to recruit the E2-ubiquitin conjugate, we generated a deletion mutant of RNF157 lacking the RING domain, which was anticipated to be ligase-dead. Then, the autoubiquitination assay was carried out by N. Schwedhelm-Domeyer, where she transfected HEK293T cells transiently with the myc-RNF157 or the myc-RNF157 $\triangle$ RING construct together with HAubiquitin plasmid. Next, the lysates were collected, denatured and immunoprecipitated with myc antibody followed by immunoblotting with HA antibody for ubiquitin. The result indicated a clear autoubiquitination response upon overexpression of wild type RNF157 but not RNF157 $\triangle$ RING (Fig 3.9a).

Additionally, I also tested if Fe65 responds to a deubiquitinase (DUB) inhibitor, Nethylmaleimide (NEM). The addition of NEM blocks DUB activity (Kapuria et al., 2010) and thus the attached ubiquitin on a substrate would be preserved. If a protein is ubiquitinated, a mobility shift using SDS-PAGE analysis should be observed with NEM treatment. Here, mouse cortical tissue was treated with either vehicle or $5 \mathrm{mM}$ NEM during lysis and subjected to immunoblotting with Fe65 and $\gamma$-Tubulin antibodies. A clear shift could be detected in the condition with NEM (Fig 3.9b), indicating that Fe65 can be indeed ubiquitinated.

Then we went on to determine if Fe65 is a substrate of RNF157. The ubiquitination assay was conducted by A. Matz to analyze if RNF157 ubiquitinates Fe65 and if the ubiquitination is ligase-activity-dependent. Moreover, the type of ubiquitin chain which was transferred to Fe65 was also investigated. For this experiment, Flag-Fe65 was expressed in HEK293T cells together with either myc-RNF157 or the myc-RNF157 $\triangle$ RING and with HA-tagged wild type ubiquitin or ubiquitin mutants K48R or K63R which are not able to form lysine (K)48 or K63-linked ubiquitin chain, respectively. Cell lysates were denatured, immunoprecipitated with Flagsepharose beads and immunoblotted with the HA antibody. The results showed that while wild type RNF157 ubiquitinated Fe65, the ligase-dead RNF157 $\Delta$ RING did not (Fig 3.9c). Moreover, 
these results also demonstrated that RNF157 ubiquitinated Fe65 via K63-linked chain as the ubiquitin-smear disappeared in the condition with K63R ubiquitin, where the formation of K63chain was abolished (Fig 3.9c).

a

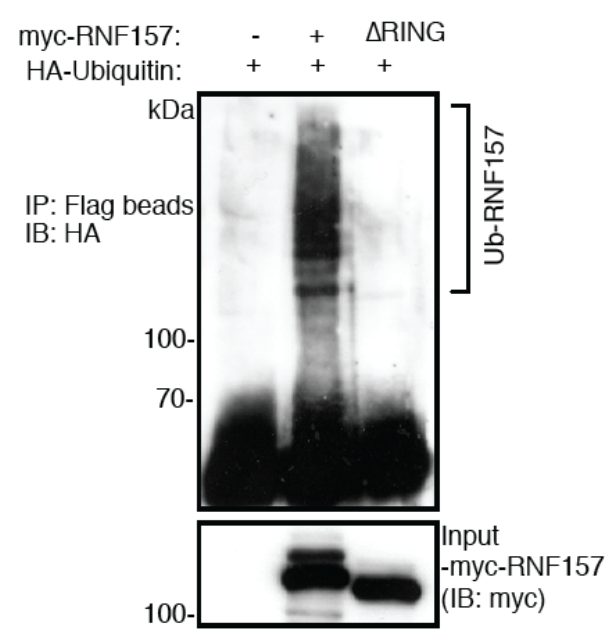

C

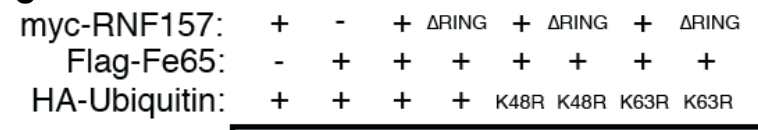

Taken together, these experiments indicated that Fe65 is ubiquitinated by RNF157, which attaches a K63-linked ubiquitin chain onto Fe65. b

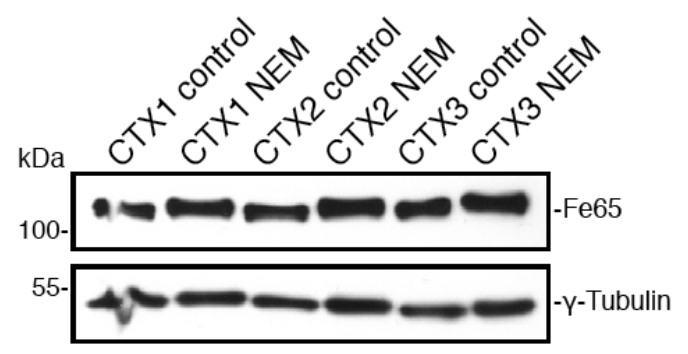

Fig 3.9 RNF157 is a RING E3 ligase and ubiquitinates Fe65.

a) HEK293T cells, transfected with mycRNF157 or myc-RNF157 $\triangle$ RING plasmid together with HA-ubiquitin plasmid, were lysed and subjected to a denaturing protocol, immunoprecipitated with myc antibody followed by immunoblotting with HA antibody. Courtesy of N. SchwedhelmDomeyer

b) Mouse cortical tissues were lysed with additional control or 5mM NEM. Lysates were immunoblotted with Fe65 and $\gamma$ Tubulin antibodies.

c) Lysates of HEK293T cells, transfected with myc-RNF157 or myc-RNF157 $\triangle$ RING construct together with Flag-Fe65 and HAtagged ubiquitin or indicated ubiquitin mutants, were denatured and subjected to immunoprecipitation with Flag-sepharose beads followed by immunoblotting with HA antibody. Courtesy of A. Matz. 


\subsubsection{Ubiquitination of Fe65 via RNF157 leads to Fe65 degradation}

Having identified Fe65 as a substrate of RNF157, I examined the consequence of the ubiquitination. Growing evidence has suggested the role of K63-linked ubiquitination in functional modification as well as lysosome-mediated degradation (Lim and Lim, 2011). However, the possibility that K63-linked ubiquitination leads to proteasomal degradation cannot be excluded (Saeki et al., 2009). Therefore, I first examined if ubiquitination of Fe65 leads to its degradation in vivo. I collected the cortical and hippocampal tissues from P5, 6 weeks and 10

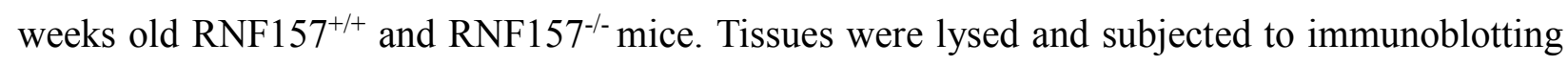
with the Fe65 antibody. While I found a slight upregulation of Fe65 in the brain of P5 RNF157-/mice, I detected a significant increase at the age of 6 weeks and 10 weeks, indicating an agedependent upregulation of Fe65 (Fig 3.10a, b). Moreover, to establish that the upregulation of Fe65 is a consequence of post-translational modifications, we further analyzed the mRNA level in the RNF157//- mice by quantitative PCR. First, I designed the primers for Fe65. Then total RNA was isolated from cortices of P5 and 10-week-old wild type and RNF157/- littermates (3 pairs). The quantitative PCR was carried out by N. Schwedhelm-Domeyer and we found that Fe65 mRNA expression remained unaffected in the RNF157/- brain (Fig 3.10c).

As mentioned in chapter 3.2.2, the localization of Fe65 influences neuronal apoptosis. Hence, I went on to examine if the accumulation of Fe65 is restricted to a certain compartment in the cell using subcellular fractionation analysis. Cortical tissues isolated from 6-week-old $\mathrm{RNF} 157^{+/+}$and RNF157/- mice were separated into the nuclear fraction (NF) and the postnuclear supernatant (PNS). Interestingly, I found that Fe65 was upregulated both in the PNS and NF (Fig 3.10d). On comparing the cytoplasmic Fe65, which also contained phosphorylated versions of Fe65, nuclear Fe65 appeared to be unphosphorylated. An additional Fe65 antibody/antigen competition experiment confirmed the specificity of these Fe65 bands (Fig 3.10e). Taken together, these results suggested that RNF157 is required for the ubiquitination of Fe65 and lossof-RNF157 results in a slow upregulation of Fe65 in the brain. 
a
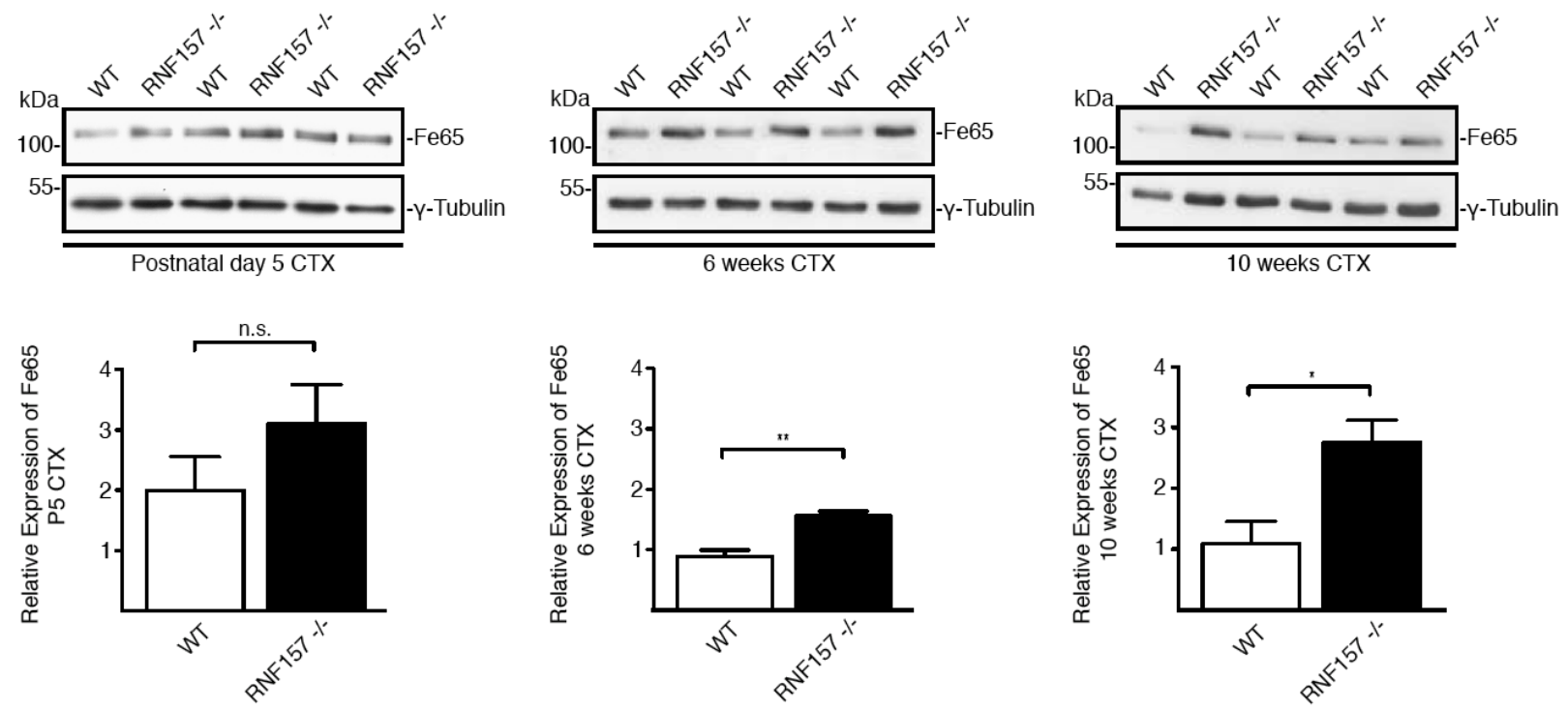

b
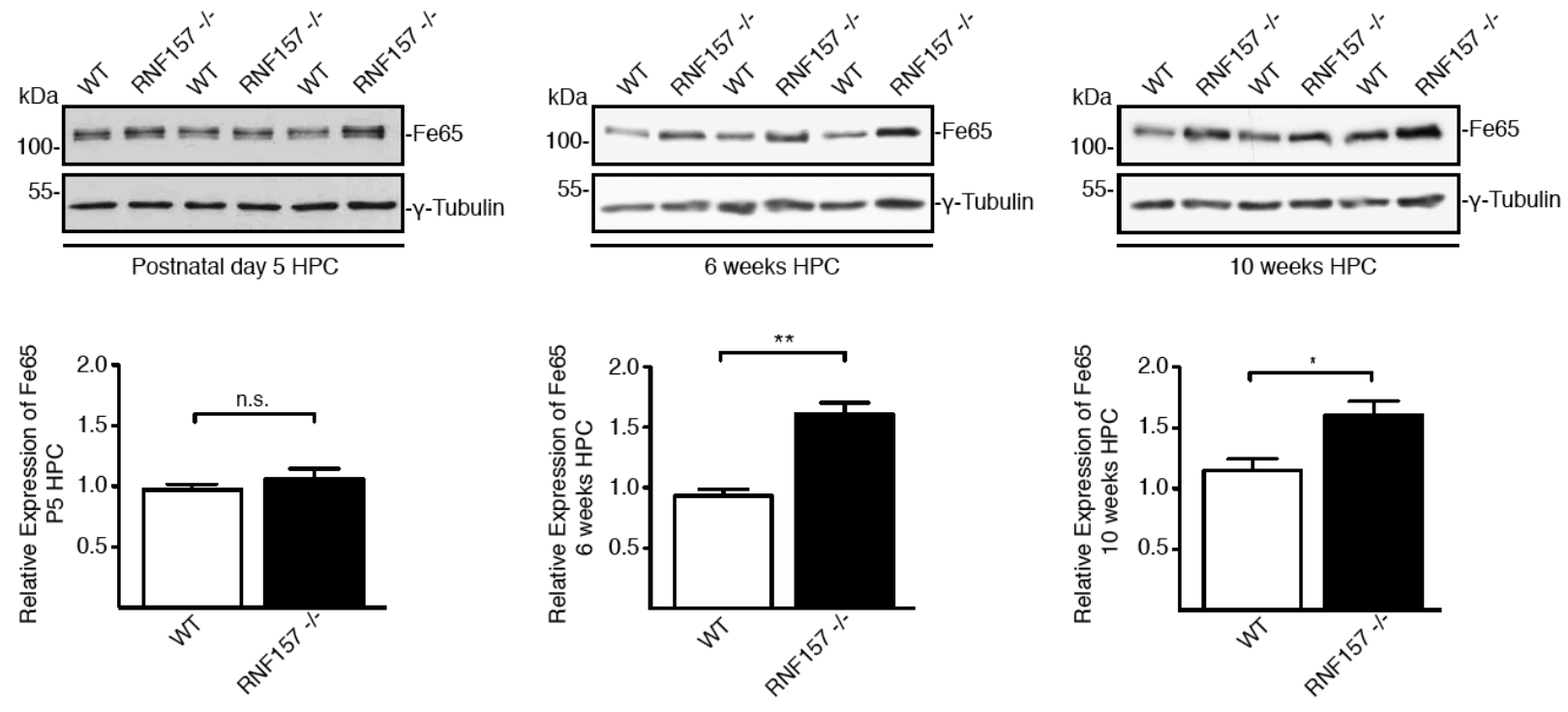

c

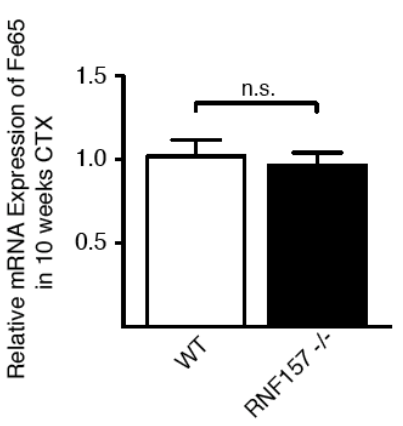

d
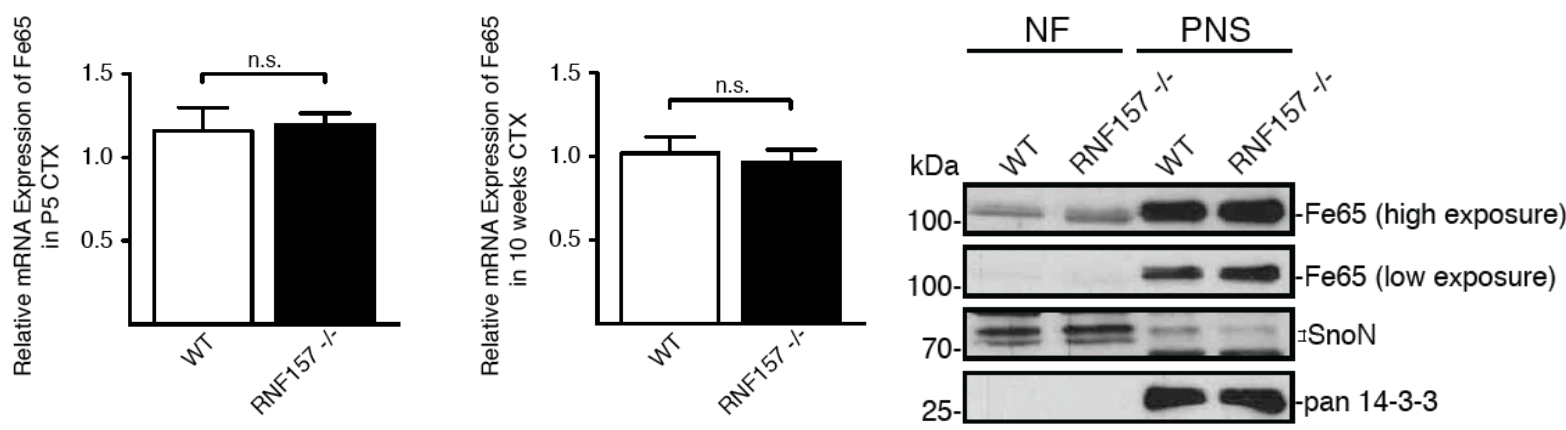
e

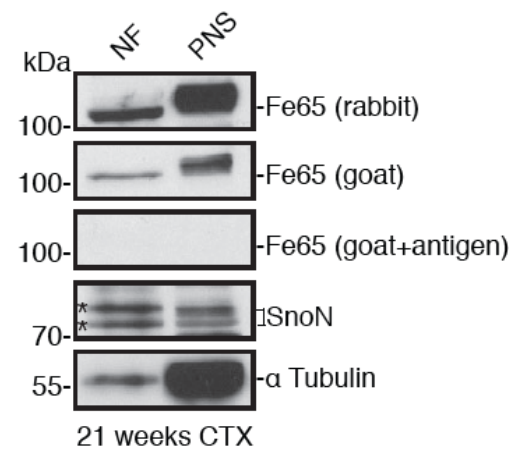

Fig 3.10 Loss of RNF157 led to slow accumulation of Fe65.

a) Cortical and b) hippocampal lysates of P5, 6-week-old as well as 10week-old wild type or RNF157 knockout mice were immunoblotted using the Fe65 antibody. $\gamma$-Tubulin served as loading control. The lower panel indicates the quantification of the Fe65 level normalized to the loading control. (Student's t-test, n.s. $=$ non-significant, $*=\mathrm{p}<0.05, * *=\mathrm{p}<0.01$ )

c) Quantitative PCR of Fe65 mRNA expression in P5 and 10-week-old cortices of wild type and RNF $157^{-/-}$mice. Three independent littermates were analyzed (Student's t-test, n.s.=non-significant).

d) 6-week-old wild type and $\mathrm{RNF} 157^{-/-}$cortices were subjected to subcellular fractionation followed by immunoblotting with the Fe65, SnoN and pan 14-3-3 antibodies. SnoN served as quality control for nuclear fraction and pan 14-3-3 for postnuclear supernatant.

e) Subcellular fractions of 21 weeks cortical tissue were immunoblotted with the rabbit Fe65 antibody, that was used throughout the paper, the goat Fe65 antibody and the goat Fe65 antibody pre-absorbed with the Fe65 antigen, SnoN and $\alpha$-Tubulin antibodies. The latter two served as quality control for nuclear fraction and postnuclear supernatant, respectively. The asterisks indicate the specific bands.

\subsubsection{The degradation of Fe65 is mediated by the proteasome}

Due to the slow accumulation of Fe65, I asked how Fe65 is degraded. Proteins are degraded via two major pathways: by either lysosomes or proteasomes (Ciechanover, 2013; Korolchuk et al., 2010). Therefore, I first tested if treatment of neurons with lysosomal inhibitors alters the protein level of Fe65. 24 hours before lysis, cultured cortical neurons were treated with either leupeptin or $\mathrm{NH}_{4} \mathrm{Cl}$. The lysates were immunoblotted with Fe65, APP (positive control for lysosomal inhibition) and $\beta$-Actin antibodies (Fig 3.11a, b). While APP accumulated in the neurons treated with lysosomal inhibitors, Fe65 level appeared to be stable. This indicates that Fe65 is not degraded by the lysosomal pathway.

On the other hand, cultured hippocampal neurons were treated with the proteasomal inhibitor, lactacystin, and subjected to immunoblotting analysis with Fe65, SnoN (positive control for proteasomal inhibition) and $\gamma$-Tubulin antibodies. Aside from the mobility shift of Fe65 upon lactacystin treatment, I also observed an increase of Fe65 levels (Fig 3.11c), which could mean that Fe65 level is regulated by the proteasome. My results indicated that Fe65 is turned over by proteasomal degradation, rather than lysosomal degradation. 
a

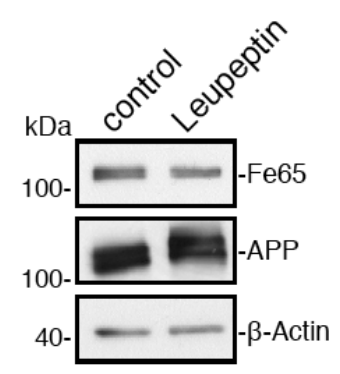

b

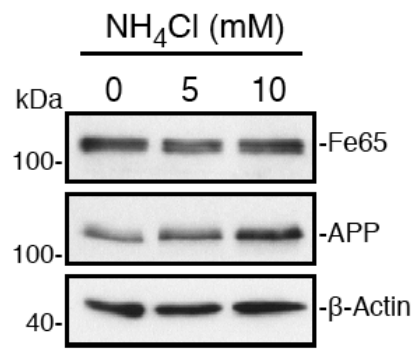

C

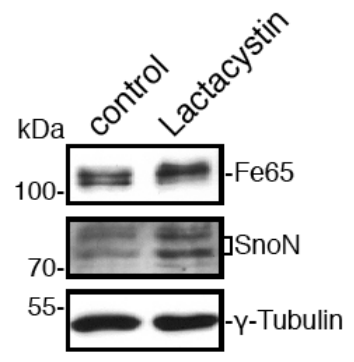

Fig 3.11 Ubiquitination of Fe65 could result in its proteasomal degradation, not lysosomal degradation.

a) Lysates of cortical neurons, treated with either vehicle or $100 \mu \mathrm{M}$ leupeptin for 24 hours, were immunoblotted with Fe65, APP and $\beta$-Actin antibodies. The latter served as loading control.

b) Lysates of cortical neurons, treated with either vehicle or indicated concentration of $\mathrm{NH}_{4} \mathrm{Cl}$ for 24 hours, were subjected to immunoblotting with Fe65, APP and $\beta$-Actin antibodies. The latter served as loading control.

c) Hippocampal neurons treated with vehicle or $40 \mu \mathrm{M}$ lactacystin for 8 hours were lysed, followed by immunoblotting with Fe65, SnoN and $\gamma$-Tubulin antibodies. The latter served as loading control.

\subsection{Potential players involved in the RNF157/Fe65 survival pathway}

To enhance our understanding of RNF157 and its interactor Fe65 in neuronal survival, we set out to identify further components, which might act in this pathway. In the following, I analyzed the expression and function of three Fe65-interacting proteins and examined if they operate together with RNF157 and Fe65 in the control of neuronal survival.

\subsubsection{RNF157 competes APP for the binding with Fe65}

One of the previously characterized interaction partners of Fe65 is the amyloid precursor protein (APP) (Borg et al., 1996; Fiore et al., 1995; Guenette et al., 1996b). Various studies implicated APP and in particular its cleavage products in the development of Alzheimer's disease (Hardy and Selkoe, 2002). The interaction between Fe65 and APP was found to affect the production of A $\beta$ (Ando et al., 2001; Sabo et al., 1999). Since RNF157 interacts with Fe65 through the PTB2 domain where APP interacts with Fe65, I first examined if RNF157 has an impact on the APP/Fe65 interaction. To address this question, I expressed wild type human APP (hAPP) and control vector or myc-Fe65 expressing plasmid together with increasing amounts of the GFP-RNF157 plasmid in HEK293T for immunoprecipitation analysis. Lysates of HEK293T were immunoprecipitated with the myc antibody and subjected to immunoblotting with the APP 
antibody. Interestingly, I found that the interaction between APP and Fe65 decreased when the amount of RNF157 increased (Fig 3.12a). In addition to wild type APP, I also examined if RNF157 has similar effect on the interaction of Fe65 and the APP carrying the Swedish mutation (swAPP), since swAPP is more prone to A $\beta$ production (Citron et al., 1994; Macq et al., 1998). I found that RNF157 outcompeted swAPP for its binding to Fe65 (Fig 3.12b).

a

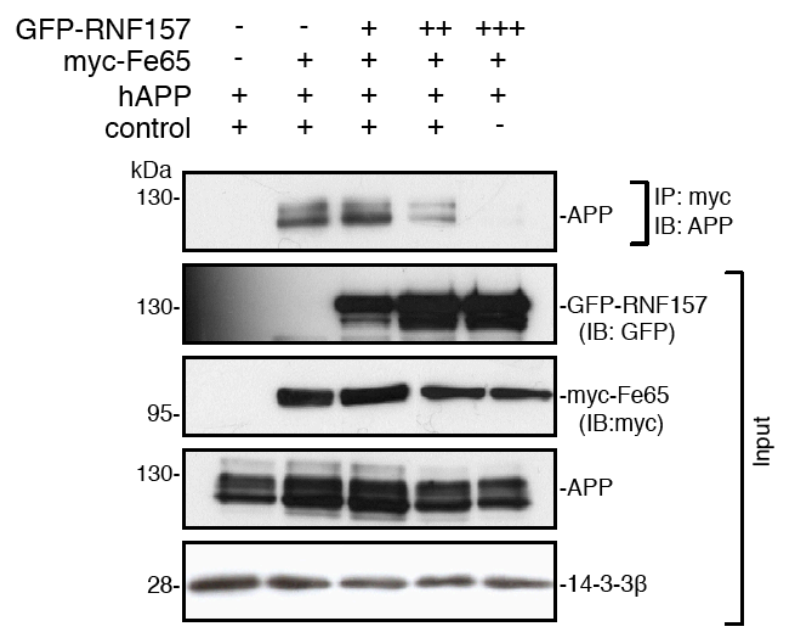

b $\begin{array}{rccccc}\text { GFP-RNF157 } & - & - & + & ++ & +++ \\ \text { myc-Fe65 } & - & + & + & + & + \\ \text { swAPP } & + & + & + & + & + \\ \text { control } & + & + & + & + & -\end{array}$

Fig 3.12 RNF157 competes APP for the interaction with Fe65.

Lysates of HEK293T cells, transfected with control vector, the myc-Fe65 plasmids, increasing amount of GFPRNF157 plasmid together with either a) hAPP or b) swAPP, were immunoprecipitated with the myc antibody and subjected to immunoblotting with the APP antibody. 14-3-3 $\beta$ serves as the loading control for APP.

With these results in mind, I reasoned that RNF157 could influence the A $\beta$ production by decreasing the binding affinity between Fe65 and APP. Since reports concerning the effect of Fe65 on A $\beta$ production were controversial and the analyses were done using different experimental setups (Ando et al., 2001; Guenette et al., 2006; Sabo et al., 1999; Santiard-Baron et al., 2005), I set out to determine if Fe65 stimulates or inhibits the cleavage of APP. In addition, to study the impact of RNF157 on the production of A $\beta$ since it competes APP for the binding with Fe65, I included RNF157 in the experiment. Here, I overexpressed either control plasmid, myc-Fe65, myc-RNF157, together with hAPP or swAPP in N2A cells and subjected the medium to an ELISA assay to examine the $A \beta_{42}$ production. The results revealed that Fe65 appeared to promote the $A \beta_{42}$ production through the cleavage of hAPP and swAPP, although the difference was not significant (Fig 3.13). Moreover, the influence of RNF157 on A $\beta_{42}$ was not consistent (Fig 3.13). Taken together, RNF157 can compete the binding of Fe65 with APP and the consequence of this binding competition needs to be further examined. 


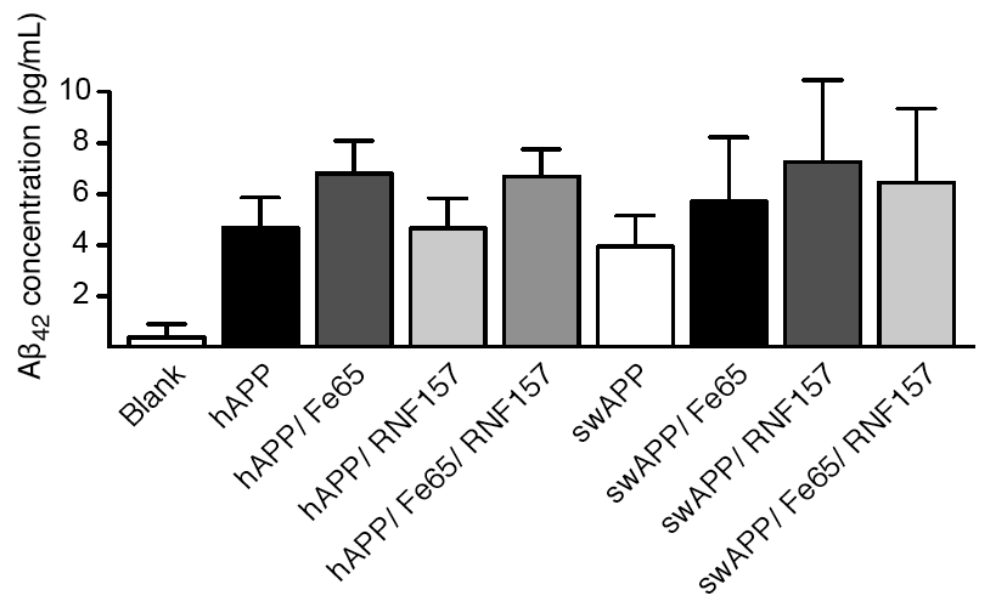

Fig 3.13 Fe65 might facilitate the production of $A \beta_{42}$ in $N 2 A$ cells.

N2A cells were transfected with control vector, myc-Fe65, mycRNF157, hAPP or swAPP plasmids and the medium was subjected to ELISA assay to determine the $\mathrm{A} \beta$ level. Blank refers to the condition of untransfected cells.

\subsubsection{Tip60, interacts with Fe65 at PTB2 domain, does not promote apoptosis}

Another interesting interaction partner of Fe65, which could be a potential player in this pathway, is Tip60. It was first identified as a HIV-1 Tat interaction protein which possesses histone acetyltransferase activity (Kamine et al., 1996; Kimura and Horikoshi, 1998; Yamamoto and Horikoshi, 1997). A previous study reported that a complex consisting of Fe65, Tip60 and amyloid precursor protein intercellular domain (AICD) can translocate into the nucleus to regulate gene transcription (Cao and Südhof, 2001). In addition, Tip60 was also shown to acetylate p53 and thus control the cell cycle arrest as well as apoptosis (Legube et al., 2004; Tyteca et al., 2006). Therefore, I examined if Tip60 is a potential player in RNF157/Fe65 pathway. First, I verified that Fe65 interacted with Tip60 using immunoprecipitation analysis (Fig 3.14a).

a

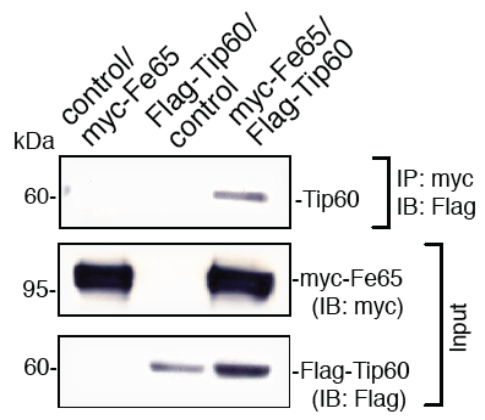

b

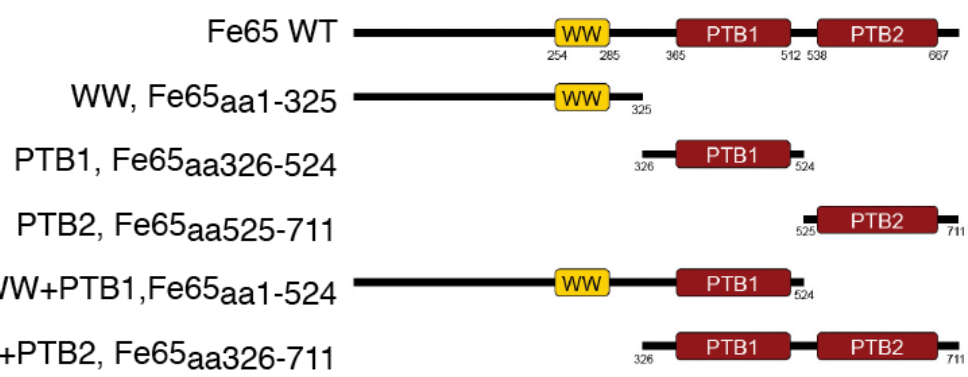


c
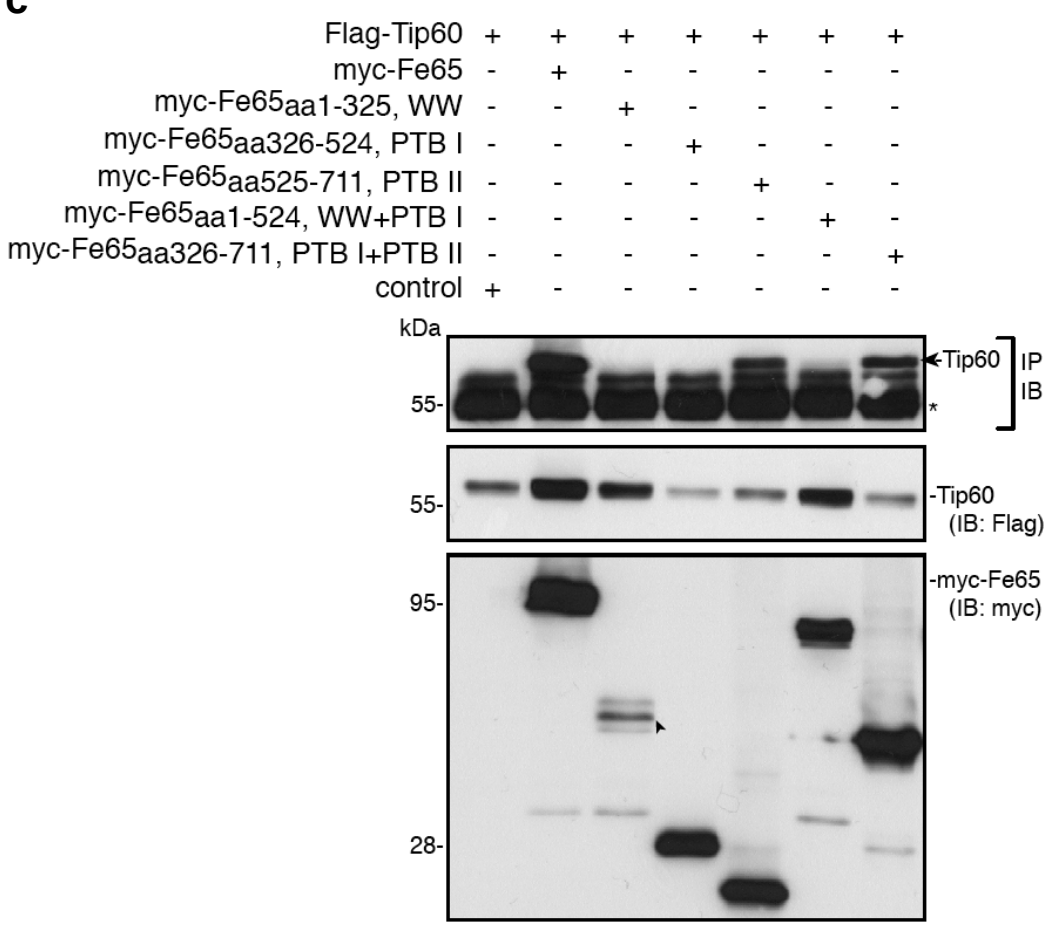

Fig 3.14 Tip60 interacts with Fe65 at the region containing PTB2 domain of Fe65.

a) HEK293T cells, transfected with control vector, together with the myc-Fe65 or the Flag-Tip60 plasmid or with both of the mycFe65 and the Flag-Tip60 plasmids, IP: mifere lysed, subjected to immunoprecipitation with the myc antibody followed by immunoblotting with the Flag antibody.

p) Domain structure of Fe65 mutants.

c) Lysates of HEK293T cells, 䱛sfected with control vector, the indicated myc-Fe65 constructs and the Flag-Tip60 plasmid, were Immunoprecipitated with the myc antibody and subjected to immunoplotting with the Flag antibody. The hrrowhead indicates the specific band and the asterisk indicates the IgG heavy chain.

Then, I determined if Fe65 interacted with Tip60 through its PTB1 domain as reported (Cao and Südhof, 2001). Using the Fe65 mutant constructs generated in the lab (Fig 3.14b), I carried out the mapping analyses. To my surprise, Tip60 did not interact with the PTB1 domain and the interaction was robust whenever the PTB2 domain was included in the expressing constructs (Fig 3.14c).

In further mapping analysis, I determined if deletion of either the PTB1, PTB2 domain or the amino acids between PTB1 and PTB2 domain abolished the interaction of Fe65 and Tip60. Hence, I generated three Fe65 mutant constructs, in which either the PTB1, PTB2 domain or the connection between PTB1 and PTB2 domain was removed (Fig 3.15a). As expected, immunoprecipitation analysis revealed that Tip60 still could bind to the Fe65 $\triangle$ PTB1 mutant although the interaction appeared to be weaker, this could be due to the lesser expression of Tip60. On the other hand, the interaction between Tip60 and Fe65 $\Delta$ PTB2 was not disturbed (Fig 3.15b). Additionally, the Fe65 $\Delta \mathrm{B} 12$ mutant still interacted with Tip60 (Fig 3.15b). These results indicate that the conformation of Fe65 or amino acid stretches adjacent to the PTB domains could be essential for the interaction between Fe65 and Tip60. 
a

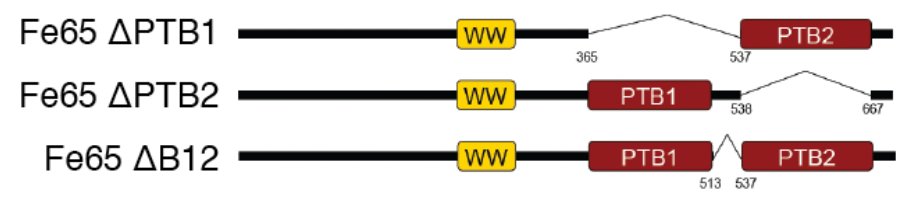

b

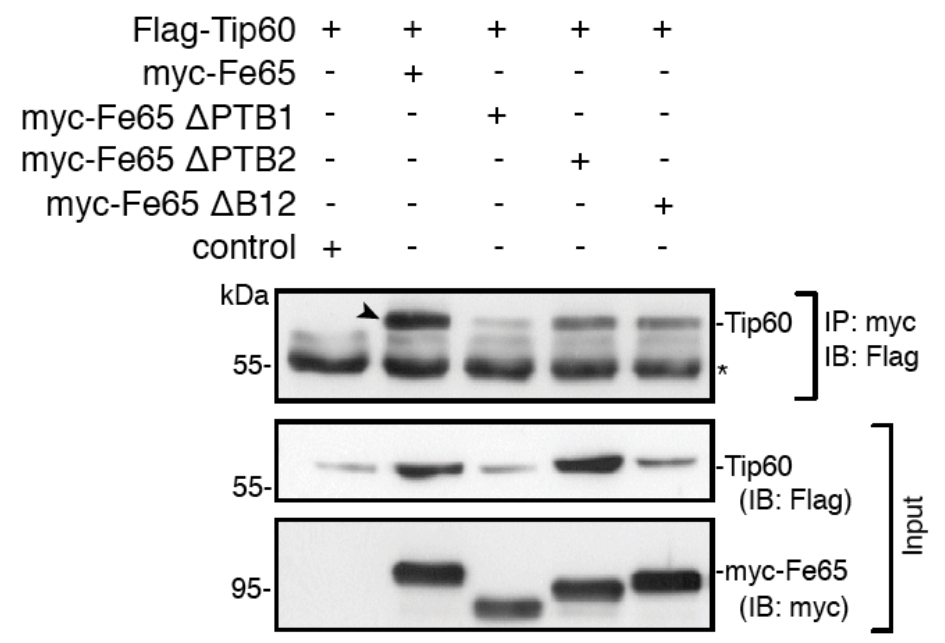

Fig 3.15 Tip60 interacts with Fe65 at the region containing $\mathrm{PTB} 2$ domain of $\mathrm{Fe} 65$.

a) Domain structure of Fe65 deletion mutants.

b) Lysates of HEK293T cells, transfected with control vector, the indicating myc-Fe65 plasmids and the Flag-Tip60 plasmid, were immunoprecipitated with the myc antibody and subjected to immunoblotting with the Flag antibody. The arrowhead indicates the specific band and the asterisk indicates the IgG heavy chain.

\subsubsection{Overexpression of Tip60 does not trigger apoptosis}

In addition to biochemical analysis of the interaction of Fe65 and Tip60, I examined the role of Tip60 in neuronal survival. Here, I overexpressed Tip60 in cerebellar granule neurons for gain-of-function analysis. The results showed that Tip60 did not induce neuronal apoptosis (Fig 3.16a). Since Tip60 was reported to form a complex together with AICD and Fe65 to regulate gene transcription (Cao and Südhof, 2001), I examined if Tip60 would further enhance neuronal apoptosis upon Fe65 overexpression. Therefore, I transfected Tip60 together with Fe65 in cerebellar granule neurons to determine the apoptotic rate. As demonstrated before, Fe65 induced neuronal apoptosis as compared to the control condition (Fig 3.16b). However, cell death was not increased when I co-expressed Tip60 on top of Fe65 expression. To sum up, Tip60 did neither induce neuronal apoptosis on its own nor did it exacerbate the effect of Fe65. 
a

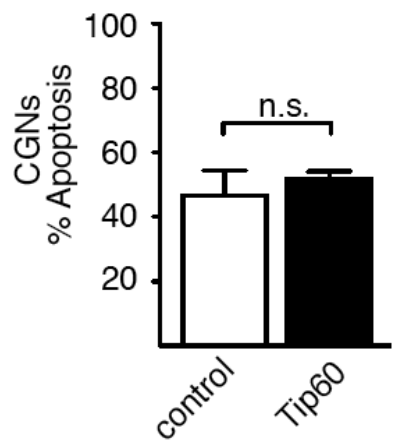

b

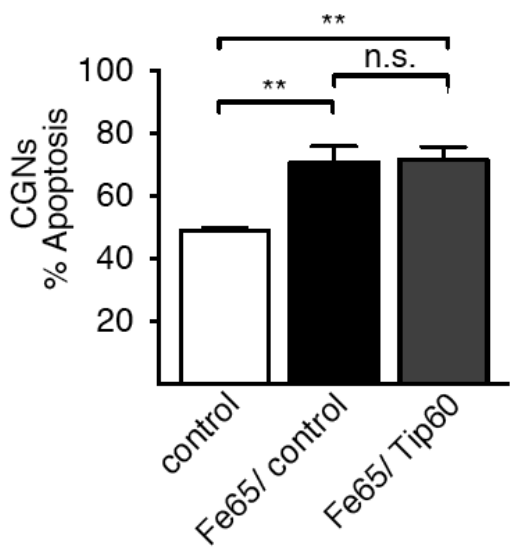

Fig 3.16 Tip60, an interactor of Fe65, does not promote neuronal death in cerebellar granule neurons.

a) Cerebellar granule neurons were transfected at DIV2 with either the empty vector or the Tip60 plasmid together with $\beta$-Gal plasmid and subjected to immunohistochemistry at DIV6 with $\beta$-Gal antibody. A total of 2434 neurons from 6 independent

experiments was analyzed (Mann-Whitney U-test, n.s.= non-significant, mean +s.e.m.).

b) Cerebellar granule neurons, transfected at DIV2 with either the control plasmid, Fe65 expressing plasmid or Fe65 and Tip60 plasmids together with $\beta$-Gal plasmid, were subjected to immunohistochemistry at DIV6 with $\beta$ Gal antibody. A total of 1376 neurons from 4 independent experiments was analyzed (ANOVA, $* *$ p $<0.01$, n.s. $=$ non-significant, mean + s.e.m.).

\subsubsection{Tip110, a novel interactor of Fe65, localizes to nucleus}

Since Tip60 is not pro-apoptotic in cerebellar granule neurons, we tried to identify another potential player in RNF157/Fe65 pathway. Since nuclear Fe65 potently induced neuronal apoptosis in neurons, I reasoned that the proteins interacting with nuclear Fe65 could have an impact on neuronal death. A yeast-two hybrid screen was therefore conducted by A. Matz using a human fetal brain cDNA library to search for nuclear interactors of Fe65. Here, we identified Tip110, a nuclear RNA-binding protein (Gu et al., 1998), as a potential interactor of Fe65. Tip110 is an HIV-1 Tat interacting protein (Liu et al., 2002) and has been implicated in RNA processing (Song et al., 2010), hematopoiesis (Liu et al., 2011), regulation of embryonic stem cells (Liu et al., 2012) as well as HIV-1-mediated gene transcription initiation and elongation (Liu et al., 2002; Zhao et al., 2014). However, the role of Tip110 in neuronal survival has not yet been investigated.

Therefore, I first confirmed the interaction of Fe65 and Tip110 in HEK293T cells (Fig 3.17a). HEK293T cells were transfected with either the HA-Tip110 plasmid or the Flag-Fe65 construct, or both of the HA-Tip110 and the Flag-Fe65 plasmids. Cell lysates were subjected to coimmunoprecipitation with the Flag antibody and immunoblotting with the HA antibody. I found that Tip110 interacted with Fe65. Next, I asked if Tip110 localizes to nucleus also in neurons. Due to the lack of a specific Tip110 antibody, I overexpressed HA-tagged Tip110 in 
hippocampal neurons and observed an expected nuclear localization (Fig 3.17b), as already shown in HeLa cells (Gu et al., 1998).

a

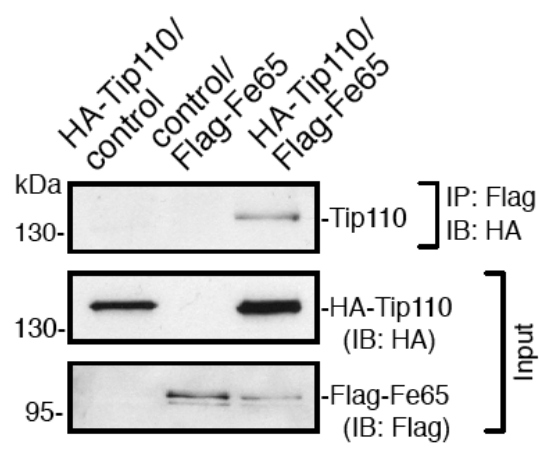

b

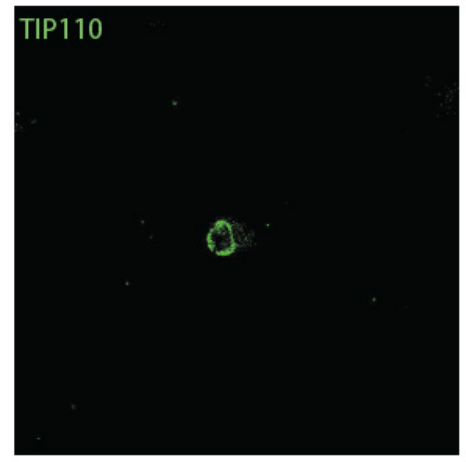

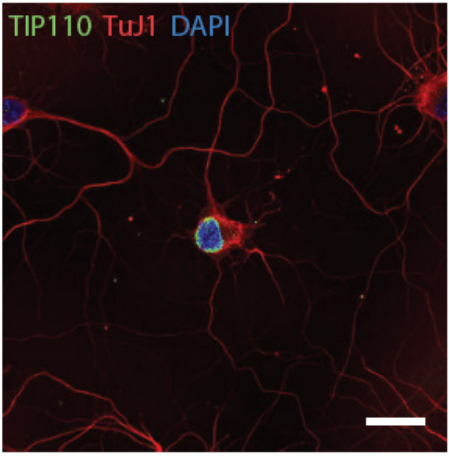

Fig 3.17 Tip110 interacts with Fe65 and localizes to the nucleus.

a) Lysates of HEK293T cells, transfected with control vector, together with the Flag-Fe65 or the HA-Tip110 plasmid or with both of the Flag-Fe65 and the HA-Tip110 plasmids, were immunoprecipitated with the Flag antibody and subjected to immunoblotting with the myc antibody.

b) Hippocampal neurons were transfected with the HA-Tip110 plasmid and subjected to immunohistochemistry with the HA antibody, the neuronal marker TuJ1 and the DNA dye DAPI. Scale bar $=20 \mu \mathrm{m}$.

\subsubsection{Tip110 promotes neuronal apoptosis}

Having established the interaction and localization of Tip110, I further asked if Tip110 has a role in mediating neuronal apoptosis. First of all, we conducted the gain-of-function analyses in cerebellar granule neurons as well as in the hippocampal neurons. Here, Tip110 was overexpressed together with $\beta$-Gal plasmid in the cultured neurons at DIV2. Neurons were fixed and analyzed at DIV6. Interestingly, overexpression of Tip110 induced cell death up to 2 folds as compared to the control cerebellar granule neurons (Fig 3.18a). In hippocampal neurons, Tip110 induced an even stronger apoptotic phenotype (Fig 3.18b, c). These results indicated that Tip110 acts in a pro-apoptotic manner in neurons. 
a

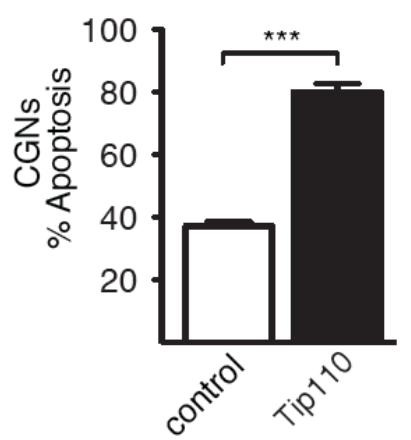

b

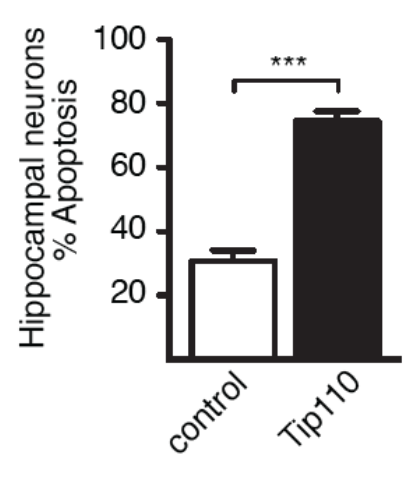

C

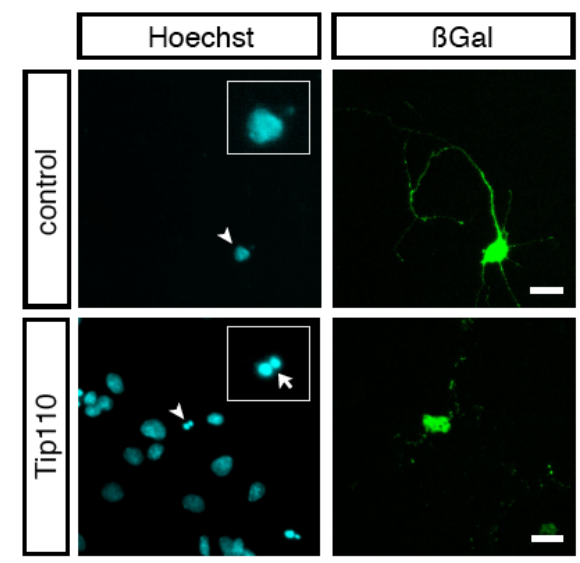

Fig 3.18 Tip110 induces neuronal apoptosis in cerebellar granule and in hippocampal neurons.

a) Cerebellar granule neurons (CGNs) transfected at DIV2 with the empty vector or the Tip110 expression plasmid together with the $\beta$-Gal plasmid were subjected to immunocytochemistry with the $\beta$-Gal antibody and the DNA dye bisbenzimide at DIV6. A total of 541 neurons from 3 independent experiments were analyzed (Student's t-test, $* * * p<0.001$, mean + s.e.m.). Courtesy of A. Matz.

b) Hippocampal neurons were transfected at DIV2 with control plasmid or the Tip110 expression plasmid together with the $\beta$-Gal plasmid and analyzed at DIV6. A total of 429 neurons from 3 independent experiments were included in the analysis (Student's t-test, $* * * \mathrm{p}<0.001$, mean + s.e.m.).

c) Representative images of neurons in $3.8 \mathrm{~b}$. Arrowhead indicates the nucleus of transfected neurons. Insets depict higher magnification of nuclei and the arrow indicates the pyknotic nucleus.

\subsubsection{Tip110 acts together with Fe65 and RNF157 in controlling neuronal survival}

To establish Tip110 as a component in the RNF157/Fe65 pathway, I first generated Tip110 RNAi constructs and verified their efficacy in HEK293T cells expressing the HA-Tip110 plasmid (Fig 3.19a). Among the four RNAi constructs only Tip110 RNAi \#3 was efficient in knocking down Tip110 and was used in the following experiments.

To investigate if Fe65 regulates neuronal apoptosis together with Tip110, I carried out epistasis experiments. Here, I overexpressed Fe65, knocked down Tip110, or expressed both the Fe65 expression plasmid and Tip110 RNAi plasmid together with the $\beta$-Gal plasmid in hippocampal neurons at DIV2. After 5 days, hippocampal neurons were immunostained with the $\beta$-Gal antibody and the DNA dye DAPI and analyzed. Interestingly, I found that while knockdown of Tip110 did not show any effect on neurons, it neutralized the apoptosis induced by Fe65 (Fig 3.19b, c). These experiments suggested that Tip110 is required for nuclear Fe65 to induce apoptosis. In further epistasis analysis, I examined if Tip110 knockdown could rescue the strong apoptosis induced by RNF157 knockdown. Here, I knocked down Tip110, RNF157 or both Tip110 and RNF157 together with overexpression of the $\beta$-Gal plasmid in hippocampal 
neurons at DIV2. Neurons were subjected to immunochemistry and analyzed at DIV7. I found that knockdown of Tip110 led to a partial rescue of the RNF157 RNAi-induced cell death (Fig 3.19d). These analyses indicated that Tip110 is a component of the RNF157/Fe65 pathway.

a

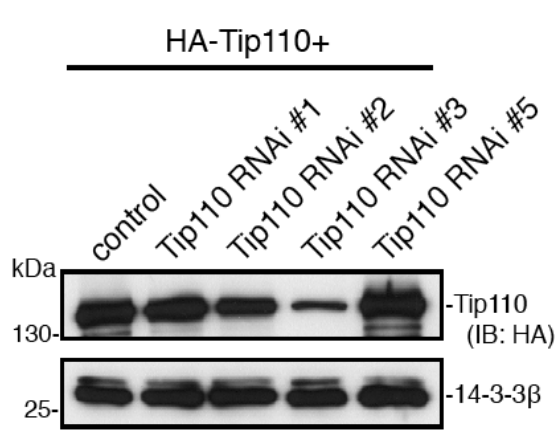

d

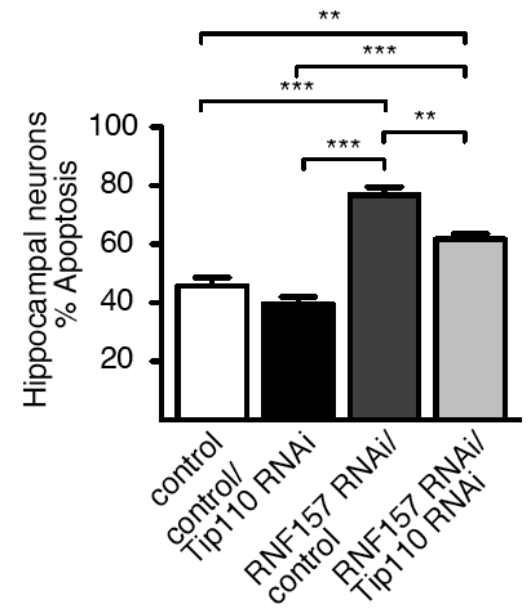

b

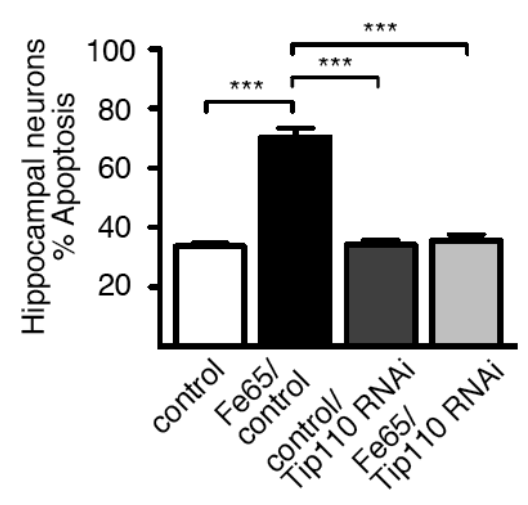

Fig 3.19 Knockdown of Tip110 rescued neuronal apoptosis induced by Fe65 and ameliorated the cell death upon RNF157 knockdown in HPNs.

a) Lysates of HEK293T cells, transfected with the HA-Tip110 plasmid together with empty vector and different Tip110 RNAi constructs, were subjected to immunoblotting with the HA and the 14-3-3 $\beta$ antibodies. The latter was a loading control.

b) Hippocampal neurons were transfected at DIV2 with control vectors, the Fe65 plasmid, the Tip110 RNAi construct or both the Fe65 expression and the Tip110 RNAi plasmids and fixed at DIV7. A total of 1447 neurons in 4 independent experiments were analyzed (ANOVA, ${ }^{* * *} \mathrm{p}<0.001$, mean +s.e.m.).

c) Representative images of neurons in 3.19b. Insets depict higher magnification of nuclei. Scale bar $=20 \mu \mathrm{m}$.

d) Hippocampal neurons were transfected at DIV2 with control vectors, the Tip110 RNAi plasmid, the RNF157 RNAi plasmid or both the Tip110 RNAi and the RNF157 RNAi plasmids and fixed at DIV7. A total of 1410 neurons in 4 independent experiments were included in the analysis (ANOVA, $* * \mathrm{p}<0.01, * * * \mathrm{p}<0.001$, mean + s.e.m.). 


\subsection{Behavioral phenotyping of RNF157 knockout mice}

According to our in vitro analyses, RNF157 has a role in neuronal survival. Therefore, loss of RNF157, which induced neuronal death, might result in neurodegeneration in mice. To determine the functional and cognitive outcome of the loss of RNF157 in vivo, I first examined the general health and activity of male wild type $(n=11)$, heterozygous $(n=22)$ and homozygous $(n=18)$ mice using the basic behavior assessment battery to rule out the influence of physical deficits on cognitive test. Then, I finalized the phenotyping with the cognitive tests to investigate learning and memory.

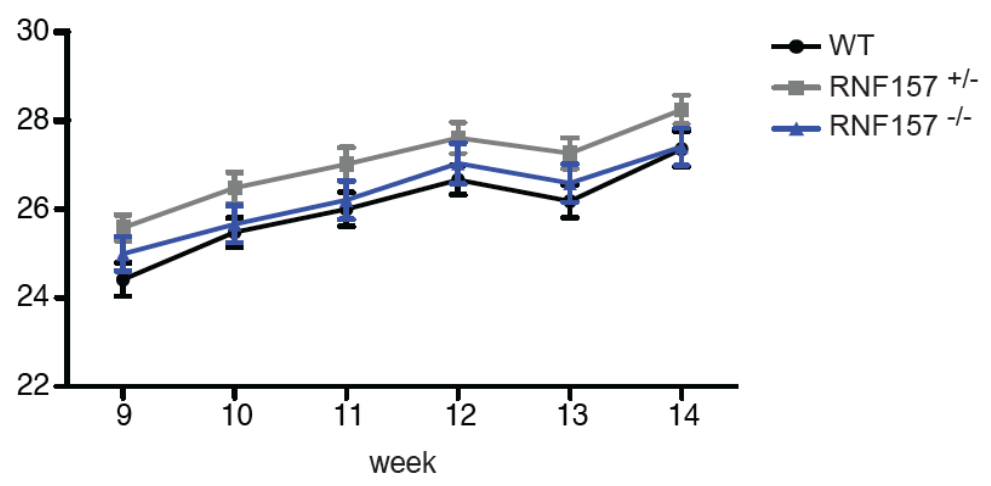

Fig 3.20 Body weight of RNF157 transgenic mice were similar in all genotypes.

The body weight measured from 9-week-old till 14-week-old did not show difference among genotypes.

\subsubsection{General behavioral characterization of RNF157 mice}

\subsubsection{Loss of RNF157 does not affect appearance and body weight}

During the behavioral tests, the general health and the weight of all mice were monitored. A previous study described that a mutation in the gene encoding the RNF157 homologue MGRN1 induces the alteration in mouse coat color (Phan et al., 2002). However, RNF157/- mice did not exhibit differences in their fur color or posture. The weight of all mice was comparable throughout the testing period (Fig 3.20).

\subsubsection{Anxiety and locomotor activity is unaltered in RNF157 $7^{-/}$mice}

First of all, both anxiety and activity of animals were assessed. Before animals got used to the testing environment, the anxiety was investigated using elevated plus maze. The plus maze consists of two open arms and two enclosed arms. By using anxiolytic or anxiogenic drugs, this 
widely used test has been proven to be a valid measure for anxiety. Untreated rodents tend to avoid the open space while the ones receiving anxiogenic substances stay even longer in the closed arms (Pellow et al., 1985; Pellow and File, 1986; Treit et al., 1993). During the test, the maze was lifted and the mice were allowed to explore the whole maze freely. The distance mice traveled and time they spent in each area (open arms, closed arms and center) were recorded. I found all tested mice exhibited natural avoidance of the elevated, open area and spent most of the time in the closed arm (Fig 3.16a; $<<0.001$ ). They also travelled farthest in the closed arm (Fig $3.16 \mathrm{~b} ; \mathrm{p}<0.001)$. However, there was no difference between genotypes in time spent (Fig 3.21a) and distance travelled (Fig 3.21b).

a

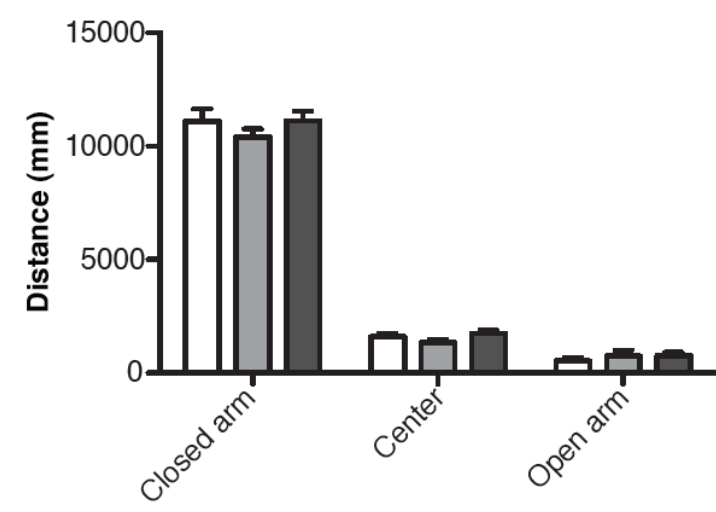

b

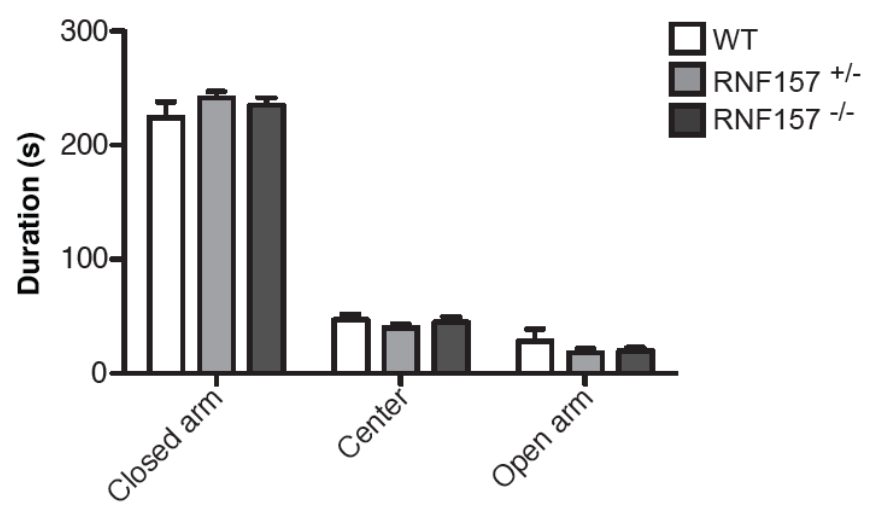

Fig 3.21 RNF157 transgenic mice exhibit normal anxiety levels in the elevated plus maze test.

a) The distance animals travelled b) the time mice spent in the closed arm, center and the open arm in the elevated plus maze were not different between genotypes.

In the open field test, all genotypes travelled significantly farther and spent significantly more time in the peripheral area as compared to the intermediate and the center zones (Fig 3.22a, $\mathrm{b} ; \mathrm{p}<0.001)$. Mice also exhibited the tendency to remain close to the walls and to avoid the central area. This behavior is referred to as thigmotaxis, which could be used as another readout for anxiety in rodents (Simon et al., 1994; Treit and Fundytus, 1988). Although the wild type mice appeared to travel more in the peripheral area than the RNF157 $7^{+/-}$and RNF157/- mice (Fig 3.22a), no overall difference could be observed in distance travelled and time spent in each zone (Fig 3.22b). The average velocity was also comparable between the different genotypes (Fig 3.22c). These results suggested that deletion of RNF157 has no impact on anxiety and general activity. 
a

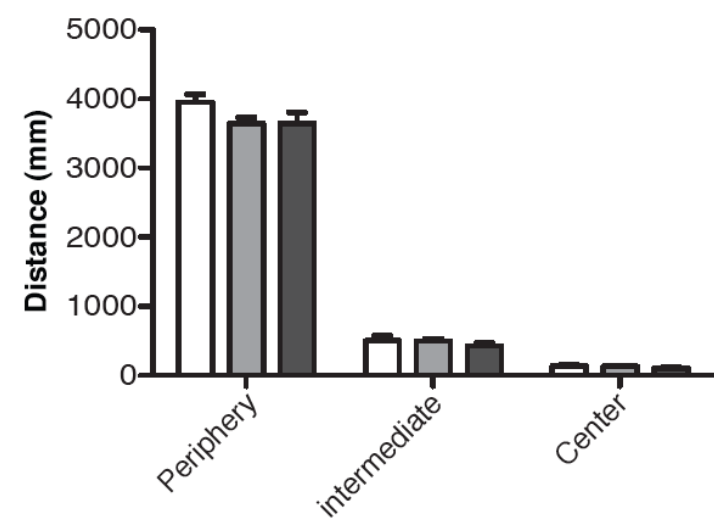

C

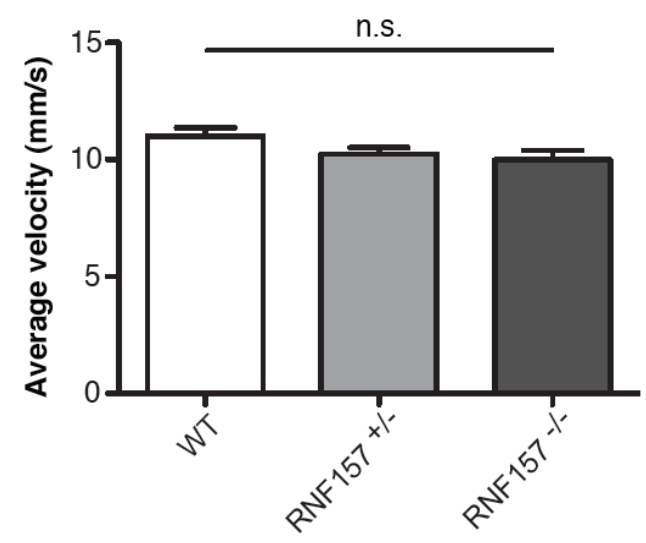

b

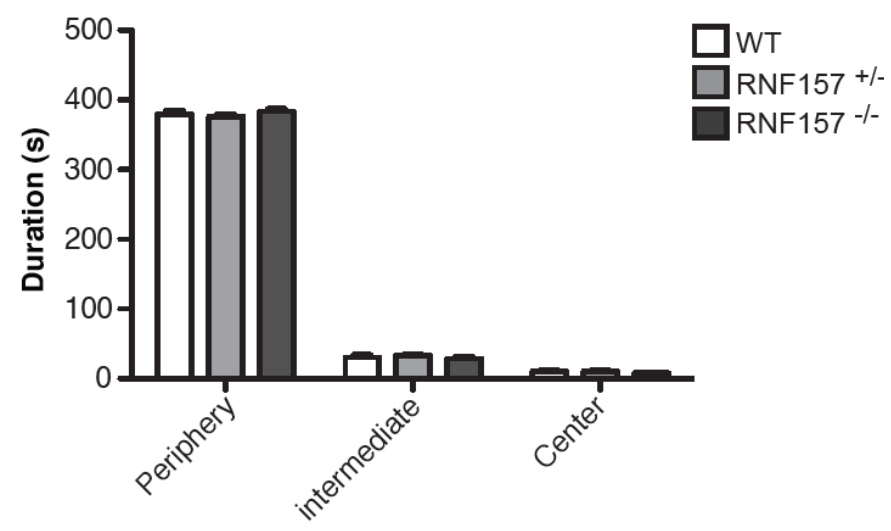

Fig 3.22 RNF157 transgenic mice showed similar locomotor activity.

a) The distance mice travelled b) the time mice spent in the closed arm, center and the open arm in the open field were similar for all genotypes.

c) No difference was shown in the average velocity with which animals were traveling in the open field.

\subsubsection{RNF157 $7^{-/-}$mice show intact motor function}

The general motor function including balance and coordination was assessed using the rotarod test. The duration that mice can stay on the accelerating rotarod is recorded. On the first test day, I observed a difference in the genotypes (Fig 3.23a; p < 0.05). Post-hoc analysis revealed that $\mathrm{RNF} 157^{-/-}$mice performed significantly worse as compared to wild type littermates (Fig 3.23a; p < 0.01). However, all mice improved the performance when tested 24 hours later. To verify this result, a three-trial rotarod test was conducted, for which the mice were tested 3 times in a day, 1 hour between each trial. No difference was found in all the trials and all mice exhibited intact motor learning (Fig 3.23b).

In addition, the muscular strength was measured using grip strength meter. The strength that animals apply to hold the grip was measured. The measurement showed that all mice possessed similar grip force (Fig 3.23c). These results indicated that the deletion of RNF157 did not affect muscle coordination and strength. 

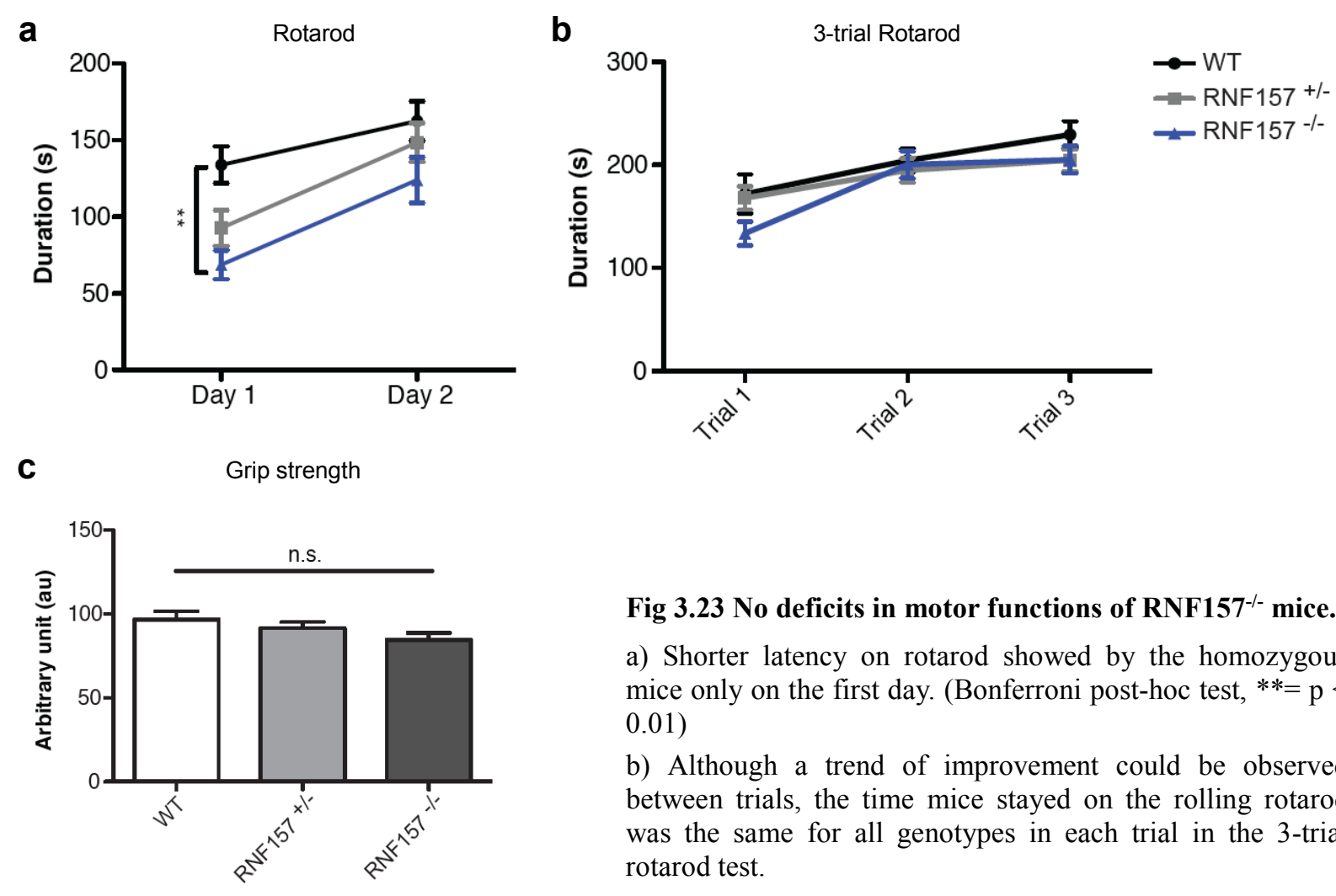

Fig 3.23 No deficits in motor functions of RNF157/- mice.

a) Shorter latency on rotarod showed by the homozygous mice only on the first day. (Bonferroni post-hoc test, $* *=p<$ $0.01)$

b) Although a trend of improvement could be observed between trials, the time mice stayed on the rolling rotarod was the same for all genotypes in each trial in the 3-trial rotarod test.

c) All test animals showed comparable grip force.

\subsubsection{Intact sensory and sensorimotor gating functions in $\mathrm{RNF} 157^{-/-}$mice}

Next, I went on to test the sensory functions including vision, hearing, olfaction as well as pain sensation since intact sensory functions are required for the following cognitive tests. First, I assessed the vision using the visual cliff test. The ground of the apparatus is divided into a ground and an air area. During the experiment, the apparatus is lifted up and the mice can see the floor through the transparent "air" area while the "ground" area is opaque. Mice tend to stay in the ground area if the depth perception is intact (Fox, 1965). I found that all mice spent more time in the "ground" area than the "air" part (Fig 3.24a; p < 0.0001) and there was no significant difference between genotypes. This result indicates an intact depth perception of all mice.

The hearing test examines the hearing ability by measuring animals' startle responses to the sound at different $\mathrm{dB}$ level. The wild type and homozygous mice responded similarly to the sounds (Fig 3.24b). The startle amplitude of heterozygous mice however was significantly stronger than that of wild type $(113 \mathrm{~dB}: \mathrm{p}<0.0001 ; 120 \mathrm{~dB}: \mathrm{p}<0.05)$ and homozygous mice (107 dB: $p<0.01 ; 110 \mathrm{~dB}: \mathrm{p}<0.01 ; 113 \mathrm{~dB}: \mathrm{p}<0.0001 ; 120 \mathrm{~dB}: \mathrm{p}<0.0001)$. These could be attributed to the trend that heterozygous mice were slight heavier as shown in Fig 3.20.

The following olfactory ability was evaluated by comparing the duration that mice need to 
find the hidden cookies. One piece of cookie was buried shallowly in the bedding in a cage as described in chapter 2.13.11. During the test, mice were released in the cage and allowed to search for the cookie. Three trials with an inter-trial interval of $50 \mathrm{~min}$ were conducted in each mouse. The cookie was visible in the $3^{\text {rd }}$ trial as a control. Mice responded significantly faster in the subsequent sessions (Fig 3.24c, p $<0.0001$ ), which implied a learning effect and I did not find a difference between genotypes. This indicates that the olfactory sensitivity is normal in all genotypes tested.

Pain sensation was examined by the duration that animals can stay on the hot plate. Mice were placed on a $55^{\circ} \mathrm{C}$ hot metal plate and the duration from placement till animals respond to the heat was recorded. Similar to the wild type mice, the heterozygous and homozygous animals were able to tolerate the heat to the same extent (Fig 3.24d). The measurement revealed that all tested mice had comparable sensitivity to pain.

a

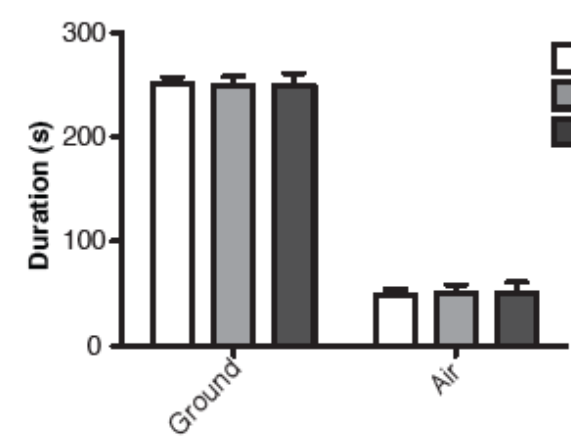

C

Olfaction b

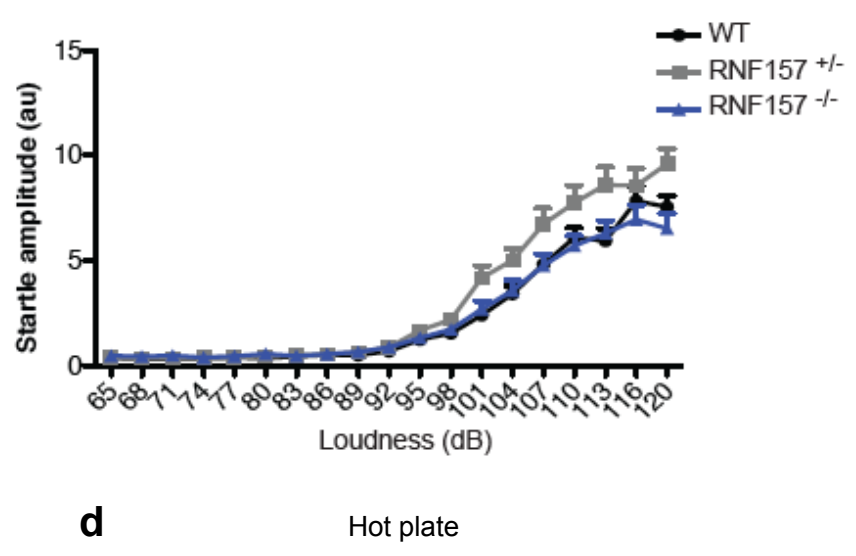

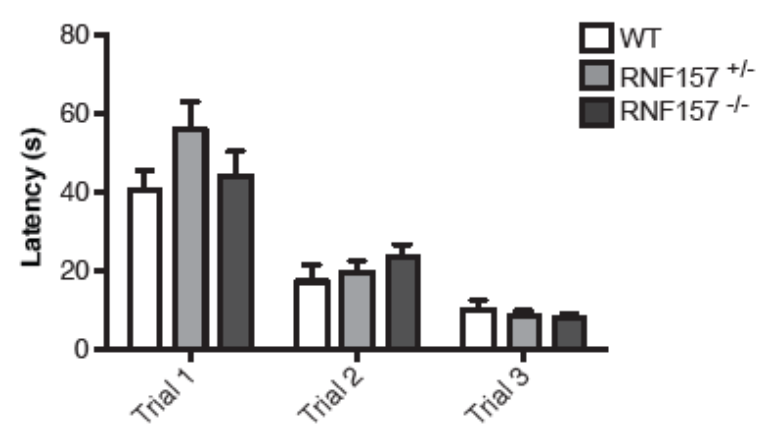

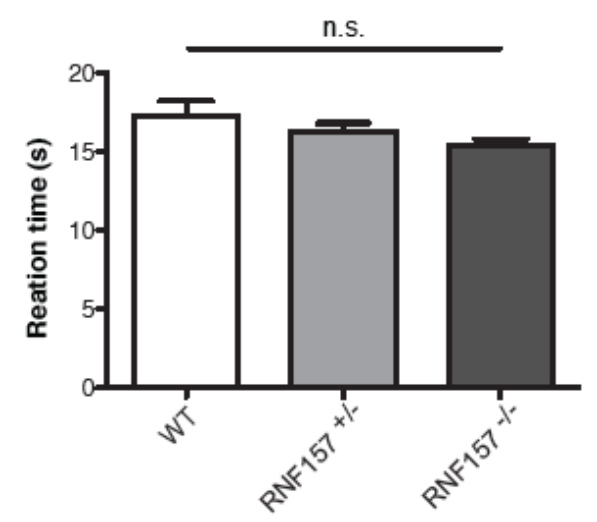


e

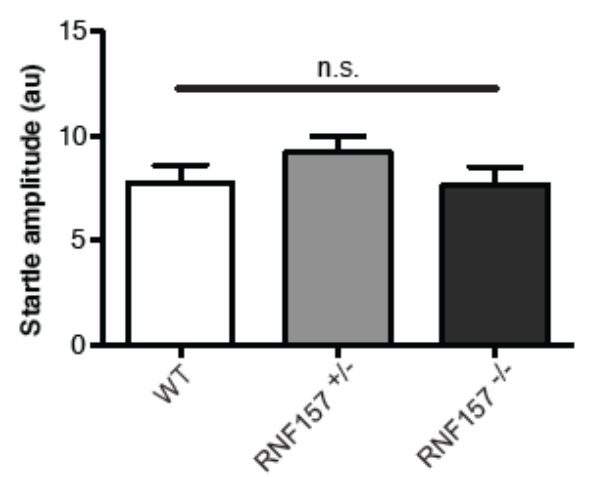

f Pre-pulse inhibition

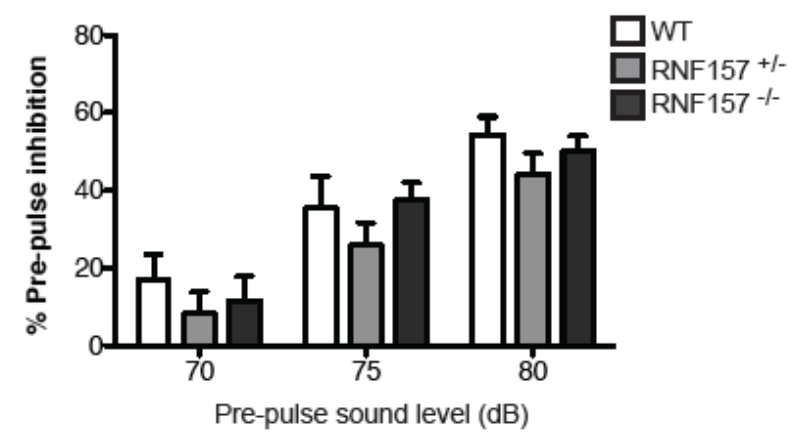

Fig 3.24 No deficits in sensory functions of RNF157 $7^{-/-}$mice.

a) The time mice spent in the "air" or "ground" part of the visual cliff test apparatus. Mice from all genotypes spent significantly more time in the "ground" area than "air" area (2-way ANOVA, $* * *=p<0.0001)$, indicating intact vision.

b) The startle amplitude plotted against the sound intensities. All mice showed a similar hearing curve, the startle amplitude was clear from $95 \mathrm{~dB}$ onwards.

c) The time that mice needed to find the hidden cookies. No difference was observed, indicating all animals had similar olfactory sensitivity.

d) The time animals need to sense the heat. All animals responded as soon as each other in the hot plate test, indicating intact pain perception.

e) The startle responses to the loud pulse as baseline for pre-pulse inhibition test. No difference in the startle amplitude could be seen between genotypes.

f) Percentage of pre-pulse inhibition plotted against the pre-pulse intensities. Mice from all three genotypes exhibited similar pre-pulse inhibition.

In addition, the sensorimotor-gating ability was examined using the pre-pulse inhibition test, in which the startle responses to auditory stimuli presented with or without a pre-pulse were recorded. Mice were placed into the metal chamber enclosed in a sound-attenuating isolation box. Various auditory stimuli were played and the startle responses of animals were recorded. Since I have already mentioned that the auditory function of all animals was undamaged, the result of pre-pulse inhibition test can indicate the integrity of the cortico-striato-pallido-pontine circuitry (Swerdlow et al., 2000). The similar startle responses to a loud stimulus demonstrated that all mice had an equal startle baseline (Fig 3.24e). Furthermore, all mice exhibited significantly stronger inhibition on the startle amplitude when the pre-pulse was louder (Fig 3.24f, $\mathrm{p}<0.001$ ). Nonetheless, no difference of the inhibition could be observed between genotypes, suggesting that the sensorimotor-gating function is unchanged upon deletion of RNF157.

To sum up, loss of RNF157 did not induce any damage on the basic sensory function such as vision, hearing, olfaction and pain sensitivity. The more complex brain circuitry underlying sensorimotor-gating was also unaltered in the $\mathrm{RNF} 157^{-/ /}$mice. 


\subsubsection{Lack of RNF157 does not affect exploratory and impulsive behaviors}

The exploratory activity was examined using the hole board test. The time mice need to make the first nose poke was recorded. Here, all mice spent approx. $100 \mathrm{sec}$ to make the first nose poke (Fig 3.25a). The number of nose pokes was also compared. While the heterozygous mice showed reduced exploratory behavior (Fig 3.25b; p < 0.05) as compared to wild type animals, the RNF157/- mice showed similar number of entries, indicating that loss of RNF157 does not alter the exploratory behavior.

Moreover, I investigated the impulsivity and the stereotypic digging behavior using the marble-burying test. The number of buried marbles in the periphery or center area was analyzed. All mice buried a similar amount of marbles in the periphery and in the center regardless of genotypes (Fig 3.25c). These data suggest that deletion of RNF157 does not affect the impulsive behaviors.

a Hole board - time to the first entry

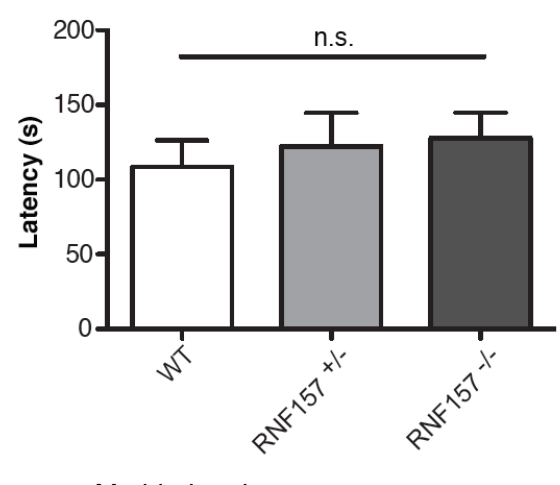

C

Marble-burying

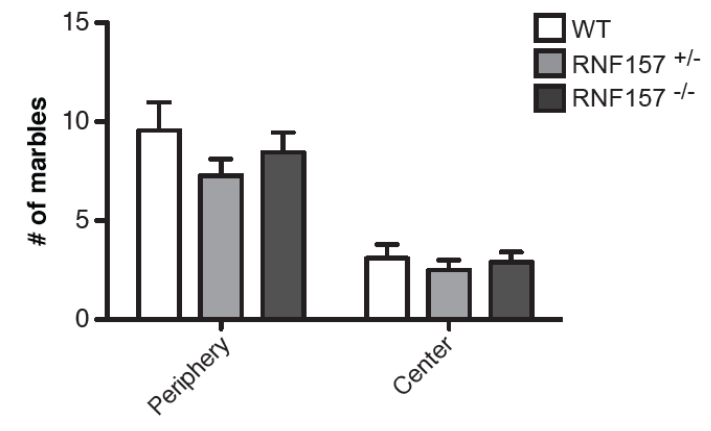

b Hole board - numbers of entry

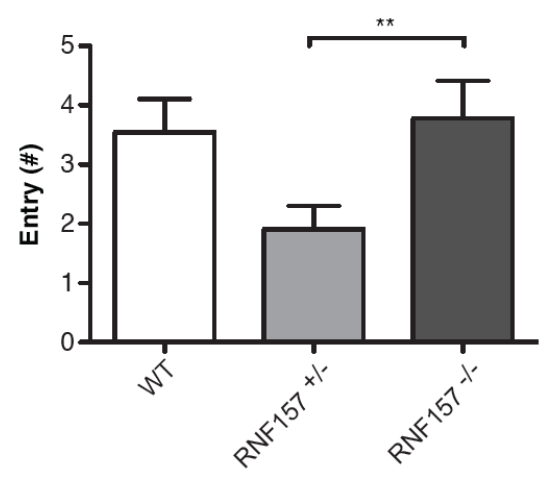

Fig 3.25 No difference in exploratory and impulsive behaviors of RNF157 knockout mice.

a) The time mice spent until mice made the first nose poke $(\mathrm{p}>0.05)$.

b) Numbers of nose pokes of each genotype in the hole board test. Although the heterozygous animals poked less times then the other two genotypes, there was no difference between the wild type and the knockout groups. (Bonferroni post-hoc test, $*=p<0.05$ )

c) The number of buried marbles was significantly different between the positions of marbles $(p<0.001)$, but not different between burying behavior of genotypes.

Taken together, the abovementioned basic behavioral battery demonstrated that all animals were indistinguishable and therefore suitable for the following cognitive tests. 


\subsubsection{Analyses of cognitive functions of RNF157 mice}

\subsubsection{Deletion of RNF157 does not impair working memory}

To assess the object recognition memory of RNF $157^{-/-}$mice, I first performed the novel object recognition test. This test is based on the natural preference of rodents for the novel objects (Ennaceur and Delacour, 1988). Animals tend to spend more time with a new object as compared to familiar objects. Various studies demonstrated the involvement of hippocampus and adjacent cortical areas in the formation and retention of object memory (Broadbent et al., 2010; Goulart et al., 2010; Hammond et al., 2004). Since RNF157 is highly expressed in the hippocampus, I hypothesized that its deletion might lead to memory deficits.

To test working memory, mice were allowed to explore the old objects for 5 sessions, 6 min per session. In the final session, a familiar object was replaced with a novel object. The time mice spent with objects was recorded. The analysis showed that all mice spent significantly more time with novel object as compared to the old ones (Fig 3.26, $p<0.001$ ). However, mice from all genotypes showed similar preference to the novel object, indicating that loss of RNF157 did not induce working memory impairment.

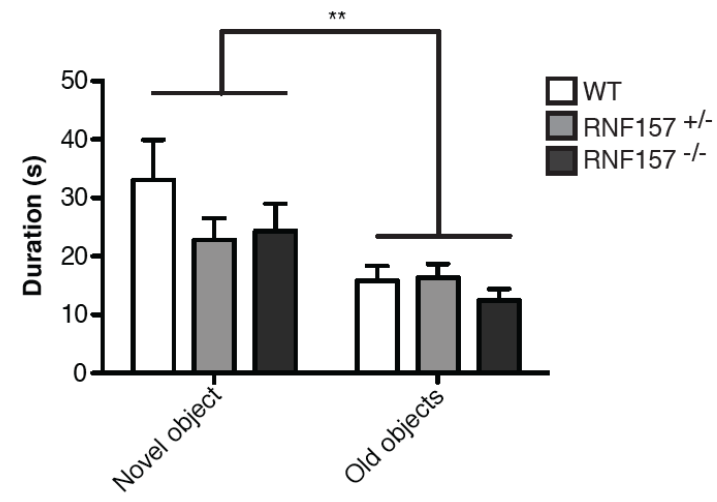

Fig 3.26 Working memory was undamaged in RNF157 transgenic mice

The time mice spent with the novel object or the old objects. Animals explored the novel object significantly longer than the old one $(p<0.001)$, but no difference between genotypes.

\subsubsection{Loss of RNF157 does not affect spatial memory}

I also examined the role of RNF157 in spatial learning and memory using Morris water maze. In rodents, the formation and consolidation of spatial memory is tightly regulated by hippocampus as well as cortex (Kolb et al., 1983; Morris et al., 1986). Due to the robust expression of RNF157 in both brain areas, I investigated if deletion of RNF157 impairs spatial memory. 
On the first two days, mice were trained to escape onto a visible platform. This was followed by 8 days of acquisition phase, in which mice search for the hidden platform. A probe trial without the platform afterwards was carried out to examine their memory. Then, the hidden platform was positioned in the opposite quadrant and the mice had 4 days to learn the new position. After the learning phase, the second probe trial was performed. For data analysis, the water maze was divided into four quadrants as depicted in Fig 3.27a: target (T), target right (TR), target opposite (TO), and target left (TL). The platform is illustrated in red. The time mice needed to escape onto the platform and their swimming distance was recorded.

The analysis showed a proper learning curve for all mice (Fig 3.27b). The escape latency was reduced over time during the first as well as the reversal training phases, indicating that all mice were able to acquire the position of the hidden platform even when the hidden platform was moved in the reversal phase. In the probe trial 1 and 2 , my data demonstrated that all animals stayed longer in the target area $(\mathrm{T})$ than other zones (Fig 3.27c, d). Interestingly, when comparing the visits onto the target, the knockout animals visited the former location platform more often than the others (Fig 3.27e, f). Although there was no main effect of the genotype in the first trial, I further analyzed the data using Bonferroni post-hoc due to the observed trend. The post-hoc analysis revealed that the knockouts made more visits than the heterozygotes $(\mathrm{p}<$ 0.001). Moreover, a main effect of genotype was observed when comparing the number of visits in the probe trial 2 (Fig 3.27f, p $<0.05$ ), indicating deletion of RNF157 led to more visits of the target. This probably could be explained by higher speed of swimming behaviors of the knockouts in the second probe trial, although all animals swam at similar speed in the first probe trial (Fig $3.27 \mathrm{~g}, \mathrm{~h}$ ). This also indicated a higher activity in the second probe trial. However, taken together, the deletion of RNF157 does not lead to impairment in spatial learning and memory.

a

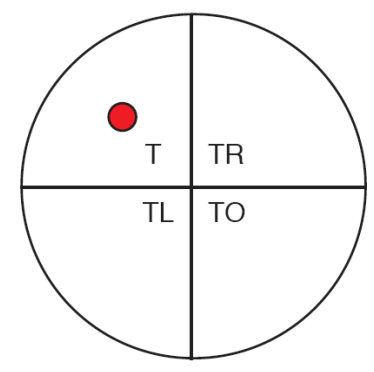

b

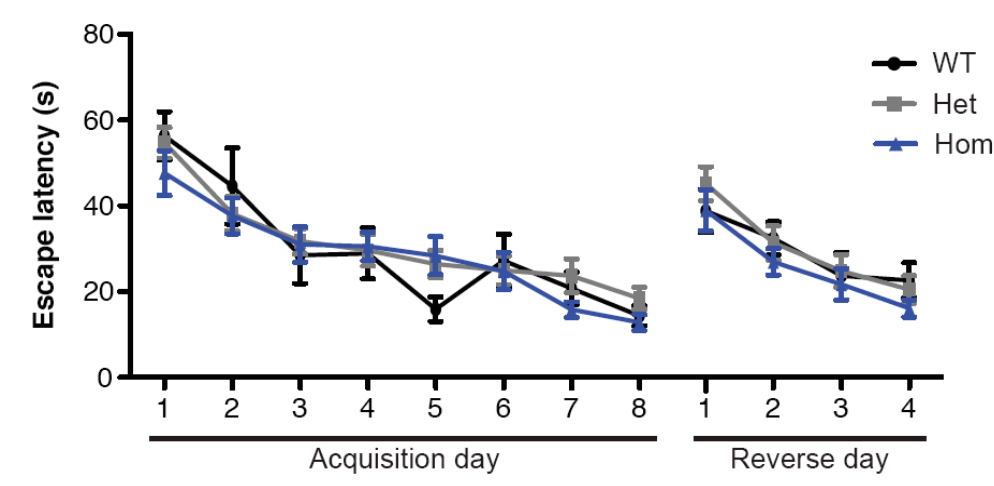


C

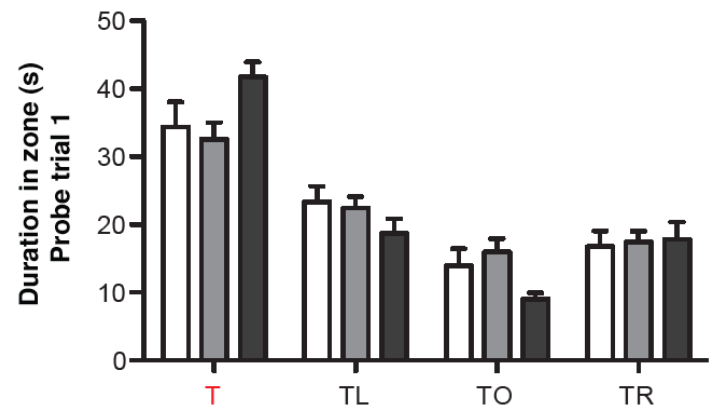

e

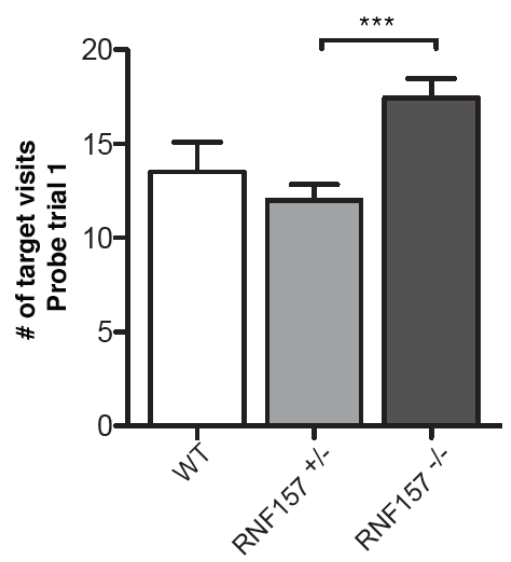

h

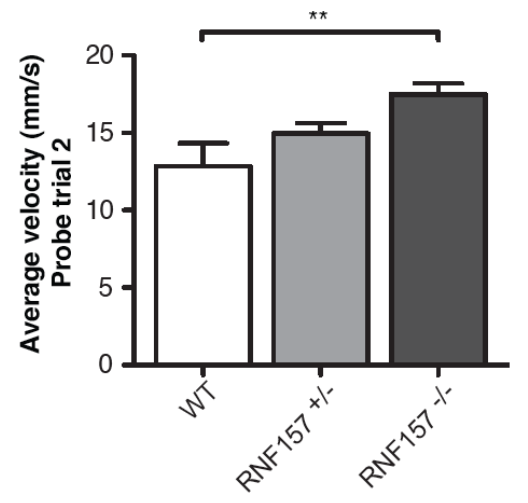
unaltered. d

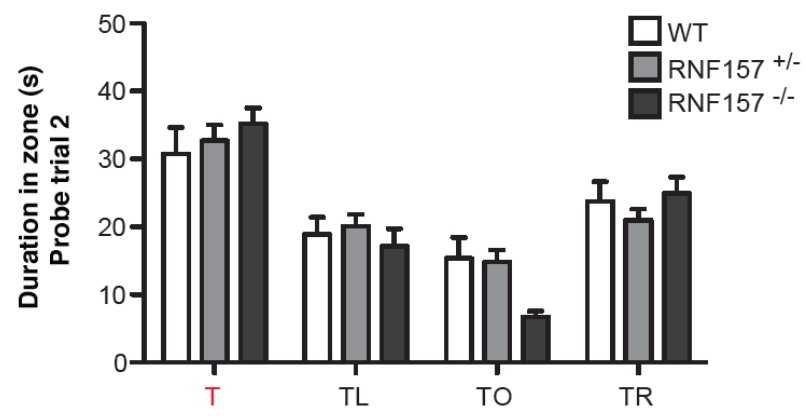

g
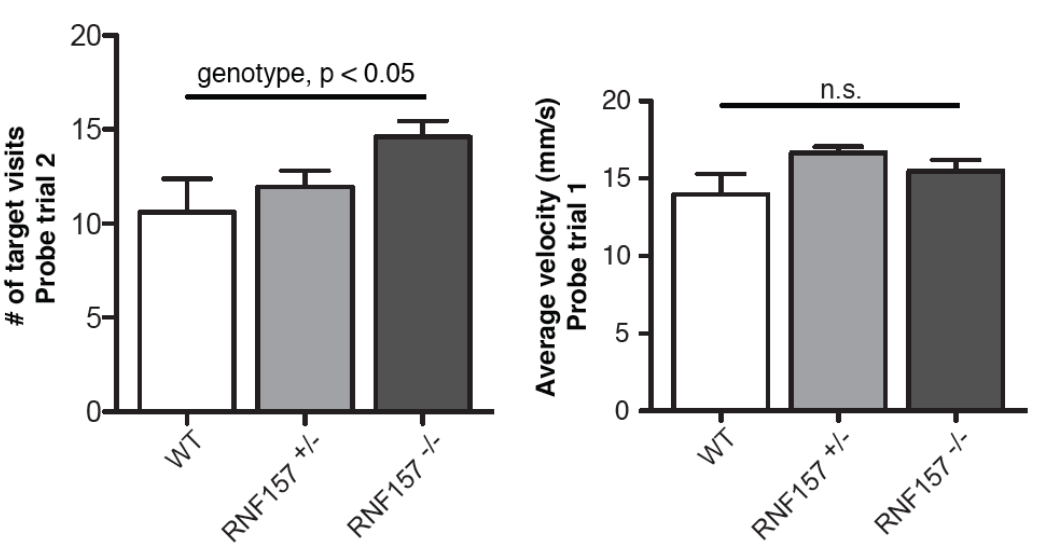

Fig 3.27 Spatial learning and memory of RNF157/-- mice is

a) Illustration of the position of the hidden platform and the division of four quadrants of the Morris water maze.

b) Curves depict the escape latency onto the platform. All mice learned equally well in the acquisition as well as reverse phases.

c), d) The time that mice swam in each quadrant during the first probe trial (c) and 2nd trial (d). The platform was removed during the trials.

e), f) Number of visits to the target in the probe trials. The knockout animals tended to visit the target more often than other mice.

f) Average swimming velocity of mice was similar in the probe trial 1 , but the RNF157/- mice swam significantly faster in the trial 2 , indicating a higher activity.

\subsubsection{RNF157 knockouts have impaired cued associative fear memory}

In addition, I investigated the impact of RNF157 on fear memory. The formation of fear memory is highly dependent on the amygdala, while the hippocampus mainly contributes to the memory retention concerning the context (Kim et al., 1993; Phillips and LeDoux, 1992). Here, I used the fear-conditioning paradigm in order to assess the role of RNF157 in fear memory.

Mice were first trained in a conditioning chamber, in which animals were exposed to a 10- 
sec tone (conditioned stimulus, CS), followed by a 2-sec foot shock (unconditioned stimulus, US). Each mouse received two tone-shock pairing so that the association between CS and US was achieved. 24 hours after training, mice were placed in the same chamber to examine their freezing behavior as the readout for contextual fear memory. Three hours later, the cued fear memory was measured. Mice were placed a new chamber and the same tone as in the training phase was played. The freezing behavior during this period refers to as cued fear memory. All mice exhibited a similar baseline freezing level in both contextual as well as cued condition (Fig $3.28 \mathrm{a}, \mathrm{b})$. Although there was no main effect of genotype on the freezing response to the same context (Fig 3.28a, p = 0.09), post-hoc test revealed that the RNF157/- mice showed less freezing as compared to the RNF $157^{+-}$mice $(\mathrm{p}<0.01)$. Interestingly, statistical analysis revealed a main effect of the genotype in the cued fear memory ( $p<0.05$, Fig 3.28b). Subsequent post-hoc tests demonstrated that the freezing level of $\mathrm{RNF} 157^{-/-}$mice attenuated significantly compared to the wild type $(\mathrm{p}<0.05)$ as well as the RNF157 $7^{+/-}$mice $(\mathrm{p}<0.01)$. In sum, impaired fear associative memory was found in the mice without RNF157. Although the deletion of RNF157 seemed to have a smaller impact on the hippocampus-based fear memory, my data suggest that RNF157 could be essential for the formation of amygdala-dependent fear memory.

a

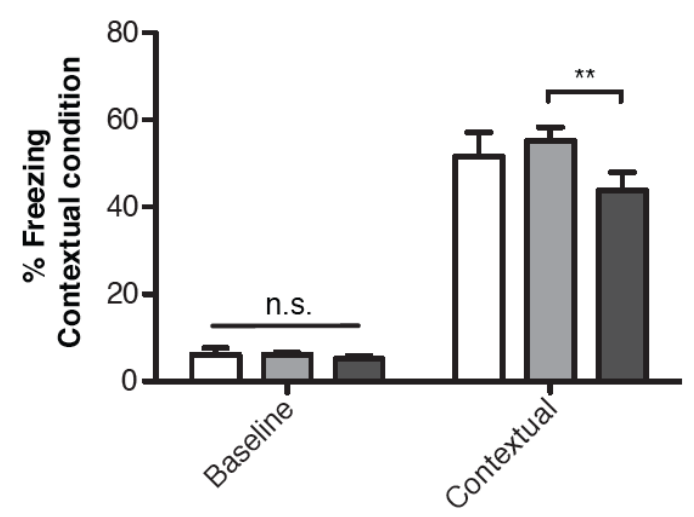

b

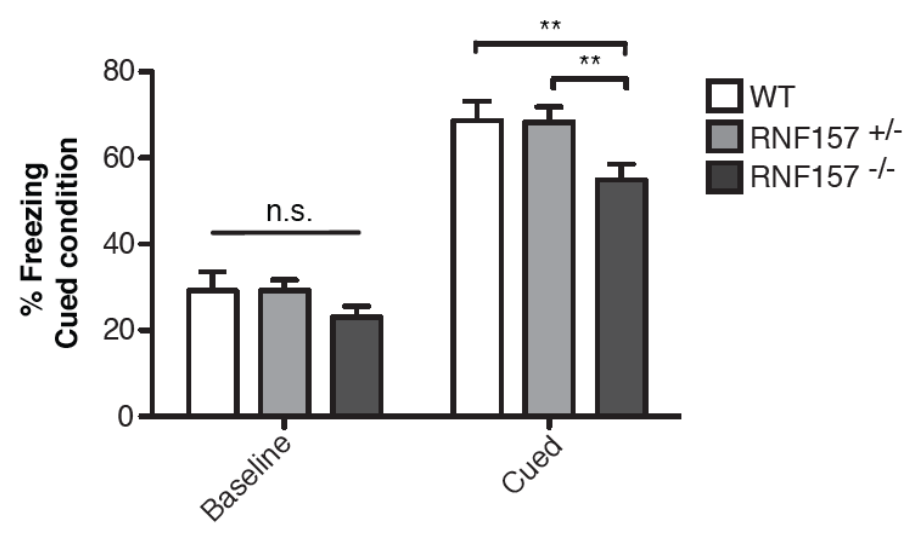

Fig 3.27 Loss-of-RNF157 leads to impairment of fear memory.

a) Freezing response to the same context. Mice showed similar baseline freezing level. While no main effect of genotype was shown $(\mathrm{p}=0.09)$, post-hoc test indicated a possible impaired fear memory in RNF157 mice as compared to RNF $157^{+-}$littermates (Bonferroni post-hoc test, $* *=\mathrm{p}<0.01$ ).

b) Freezing response in the cued fear memory test. All mice had comparable baseline freezing level. RNF157-/mice showed damaged fear memory compared to the wild type as well as heterozygous littermates (Bonferroni post-hoc test, $* *=\mathrm{p}<0.01 ; *=\mathrm{p}<0.05)$.

To sum up, the results from the aforementioned cognitive tests pointed out that loss-ofRNF157 indeed disturbs cognitive functions to some extent. Although the impact of RNF157 on 
hippocampal functions was not yet clear in adult mice, impairment in amygdala-mediated fear memory could already be observed. This indicates that RNF157 is essential for amygdaloid function in adult mice. 


\section{Discussion}

The loss of neurons in the brain is often associated with an imbalance of the protein homeostasis. Emerging evidence signifies the direct role of E3 ubiquitin ligases in neurodegeneration. A previous study demonstrated that disrupted transcription of the Mgrn1 gene induces progressive spongiform neurodegeneration (He et al., 2003). In our lab, the E3 ligase RNF157, which is highly homologous to Mgrn1 (Jiao et al., 2009), has been found to regulate neuronal survival (Matz et al., in press). Furthermore, a novel interactor of RNF157, Fe65, was found to operate in the nucleus to promote apoptosis and to act downstream in the RNF157 survival pathway (Matz et al., in press). My study further elaborated on the role of RNF157 in regulation of neuronal survival. I uncovered that aside from cerebellar granule neurons, the RNF157/Fe65 survival pathway is also active in hippocampal neurons. Moreover, my results indicate that Fe65 is a substrate of RNF157 and that Fe65 is most likely targeted for proteasomal degradation. Furthermore, I identified an additional player, the RNA-binding protein Tip110, in this survival pathway. Tip110 is required for Fe65 to trigger apoptosis in the nucleus. In addition to the in vitro characterization of RNF157, I investigated the behavior of transgenic RNF157 mice. The RNF $157^{-/-}$mice are apparently normal and exhibit intact motor and sensory functions. Notably, while all tested mice possess similar short-term and spatial working memory, mice lacking RNF157 have impaired fear-memory. To sum up, my study elucidated the RNF157/Fe65/Tip110 survival pathway in vitro. Together with the behavioral data, my work expands our understanding of the E3 ligase RNF157 in regulating neuronal survival.

\subsection{RNF157 is a brain-dominant protein involved in the Akt- and Erk- mediated survival pathways}

My study revealed that RNF157 is expressed in cultured neuronal as well as non-neuronal cells in the brain. Interestingly, RNF157 is strongly expressed in the cortex as well as hippocampus of the adult mouse brain, indicating its potential role in cognitive functions. However, only a low expression level can be observed in the internal granule layer of the cerebellum, where the cerebellar granule neurons (CGNs) are, and in white matter, while RNF157 is robustly expressed in cultured CGNs, astrocytes as well as oligodendrocyte precursor cells. Since the cultured cells are obtained from prenatal and neonatal animals, it can be 
speculated that RNF157 is essential for the early development of these cells rather than for the maintenance of the cells in the adult brain.

Using subcellular fractionation analysis, I found that RNF157 is restricted to the cytoplasm. This subcellular localization is different from that of Mgrn1, which shows a nuclear and cytoplasmic expression (Kim et al., 2007a). Unlike the widespread distribution of its homologue Mgrn1, RNF157 exhibits a brain-dominant and cytoplasmic expression, indicating a distinct role of RNF157 in the brain, but a certain degree of functional redundancy provided by Mgrn1 cannot be excluded due to the spatially overlapping distribution of Mgrn1 and RNF157.

Notably, the major survival pathways mediated by the kinases Akt and Erk in the RNF157/brains were altered due to loss of RNF157, suggesting a potential role of RNF157 in the regulation of neuronal health. To protect neurons from death after stroke, the activity of Akt has been shown to be transiently upregulated (Zhao et al., 2006). Transient activation of Erk to inhibit apoptosis can also be observed in cells responding to pro-apoptotic stimuli such as radiation, osmotic stress or growth factor withdrawal ( $\mathrm{Lu}$ and $\mathrm{Xu}, 2006 \mathrm{a}$ ). These indicate a neuroprotective function of Akt and Erk in response to cellular stress. While the total amount of Akt and Erk remains unchanged in the RNF157/- brain, the activity of both kinases decreases. This downregulation of Akt and Erk activity, however, does not affect the viability of the mice. It can be speculated that the low kinase activity is sufficient for the basic physiological functions, but mice lacking RNF157 might be more susceptible to harmful stimuli or events that are associated with aging. To test this hypothesis, further experiments using cultured neurons exposed to different stress stimuli can be considered. Moreover, it remains unclear how RNF157 regulates Akt or Erk. So far, the kinases phosphoinositide 3-kinase (PI3K) or 3-phosphoinositidedependent kinase (PDK) and the Ras/Raf/Mitogen-activated protein kinase kinase (MEK) cascade has been shown to phosphorylate Akt and Erk, respectively (Song et al., 2012). It would be worthwhile to investigate if their activity is modulated by RNF157.

\subsection{RNF157/Fe65 pathway regulates apoptosis in hippocampal neurons}

RNF157 has been shown to regulate neuronal survival in cerebellar granule neurons (Matz et al., in press). Owing to its robust expression in the hippocampus, I characterized this role of RNF157 in hippocampal neurons and found that knockdown of RNF157 triggers apoptosis in hippocampal neurons, indicating that RNF157 is pro-survival in different types of neurons. 
Furthermore, it has been demonstrated that Fe65 triggers cell death in cerebellar granule neurons (Matz et al., in press). My result showing that Fe65 also initiates apoptosis in hippocampal neurons is in line with the previous finding. In addition, it has been reported that only nuclear Fe65 contributes to cell death in the cerebellar granule neurons (Matz et al., in press). Interestingly, I found that in hippocampal neurons, both nuclear and cytoplasmic Fe65 can induce apoptosis with the nuclear Fe65 being more potent. It can be postulated that hippocampal neurons are more sensitive to Fe65-induced cellular stress, and therefore even cytoplasmic Fe65 is sufficient to cause cell death. It could be that overexpression of cytoplasmic Fe65 leads to the translocation of endogenous Fe65 into nucleus and thus to an apoptotic response in hippocampus. Another possible explanation would be that the cytoplasmic Fe65 triggers distinct apoptotic pathways. As an adaptor protein, Fe65 has been reported to bind to different transmembrane receptors such as LRP or ApoEr2 (Borquez and Gonzalez-Billault, 2012), which have been implicated in apoptosis (Hashimoto et al., 2000; Kysenius et al., 2012). Hence, it would be interesting to investigate if Fe65 governs two distinct pro-apoptotic pathways in the different cell compartments.

In addition, epitasis analysis demonstrated that Fe65 knockdown does not have an effect on neuronal apoptosis but, together with RNF157 knockdown, Fe65 knockdown rescues hippocampal neurons from cell death. Together with our previous study, regardless of neuronal cell types, RNF157 operates upstream of Fe65 in a linear pathway to control neuronal survival.

\subsection{RNF157 ubiquitinates Fe65 via K63 chains for progressive proteasomal degradation}

Since Fe65 acts downstream of the E3 ligase RNF157, I reasoned that RNF157 can ubiquitinate Fe65. My study revealed that Fe65 is ubiquitinated by RNF157 via K63-linked polyubiquitin chains. Generally, K48 polyubiquitin chains are considered as the primary signal for proteasomal targeting, while K63 chains are often associated with protein stabilization and transportation, DNA repair and lysosomal degradation (Komander and Rape, 2012). However, Saeki and colleagues have shown that K63 chains can also mediate proteasomal degradation (Saeki et al., 2009). Upon deletion of RNF157 in vivo, I found progressive accumulation of the Fe65 protein in the cortices as well as hippocampi of RNF157/- mice, while the mRNA level was unaltered, indicating that post-translational modification mediated by RNF157 leads to degradation of Fe65. 
Furthermore, I have verified that Fe65 is localized to the cytoplasm as well as the nucleus. Various studies have demonstrated that Fe65 can translocate into the nucleus together with AICD to mediate gene transcription (Bruni et al., 2002; Cao and Südhof, 2001; Kimberly et al., 2001) and K63-linked polyubiquitination has been shown to facilitate the nuclear translocation of substrates (Geetha et al., 2005). Since nuclear Fe65 is the main contributor in inducing neuronal apoptosis, I reasoned that the ubiquitination of Fe65 via RNF157 could lead to its accumulation in the nucleus to trigger apoptosis. The subcellular fractionation study, however, revealed that not only the nuclear Fe65 but also cytoplasmic Fe65 accumulate in the cortex of 10-week-old RNF157//- mice, suggesting that RNF157 ubiquitinates Fe65 mainly for degradation, not for nuclear translocation.

Next, I analyzed if Fe65 undergoes proteasomal or lysosomal protein turnover since it has been reported that K63-linked chains enhance endosomal trafficking of the substrate to the lysosome for degradation (Lauwers et al., 2009; Tofaris et al., 2011). I found that Fe65 is degraded by the proteasome rather than the lysosome. This is an interesting finding since previous studies on this matter show conflicting results. It has been shown that all ubiquitin chains except K63 chains can target substrates for proteasomal degradation (Bedford et al., 2011; $\mathrm{Xu}$ et al., 2009b). This was partial debated by Saeki and colleagues who showed that K63 chains do mediate proteasomal turnover (Saeki et al., 2009). More recently, a study reported that while K48 and K63 chains binds equally well to the proteasome, K63-chains-associated factors weaken its association with the proteasome (Nathan et al., 2013). Another possible scenario is that other E3 ligases are also involved in the ubiquitination and degradation of Fe65. Since Fe65 is an adaptor protein involved in numerous cellular signaling pathways, it can be speculated that RNF157 is not the only E3 ligase for Fe65. Notably, the homologue of RNF157, Mgrn1, has been found to interact with Fe65 (Matz et al., in press), it is very likely that Mgrn1 also ubiquitinates Fe65. In addition, Nedd4-2, a HECT-type E3 ligase belonging to Nedd4 family has been shown to ubiquitinate Fe65 (Lee et al., 2009a), although the functional implication of this is not yet known. Furthermore, it has been reported that Fe65 forms a trimeric complex with Notch1, a transmembrane receptor, and Itch, another HECT-type E3 ligase, to stimulate the ubiquitination of Notch1 by Itch probably for proteasomal degradation (Kim et al., 2012). However, it is unclear if Fe65 is turned over together with Notch1. Hence, it would be important to investigate the consequence of Mgrn1 or Nedd4-2-mediated ubiquitination of Fe65.

To sum up, my study so far suggests that RNF157 interacts with Fe65 to ubiquitinate Fe65 via K63 polyubiquitin chains and most likely targets Fe65 for proteasomal degradation in a noncanonical manner. 


\subsection{RNF157 competes with APP for the binding of Fe65}

Fe65 binds to APP via its PTB2 domain and mediated the trafficking and processing of APP (Borquez and Gonzalez-Billault, 2012; McLoughlin and Miller, 2008). Since one of the products of APP processing, A $\beta$, appears to be neurotoxic (Kriem et al., 2005; Morishima et al., 2001), I reasoned that APP might play a role in the RNF157/Fe65 survival pathway. Moreover, APP harboring Swedish mutation (swAPP) produces more $\mathrm{A} \beta$ as compared to the wild type human APP (hAPP) (Citron et al., 1992). Therefore, I included both hAPP and swAPP into my analysis and found that RNF157 can compete with both hAPP and swAPP for the binding to Fe65 via its PTB2 domain and thus interfere their interaction. This scenario resembles the one of the adaptor protein Dab1 that competes with Fe65 for the binding to APP, and in turn blocks the association between Fe65 and APP, leading to suppression of APP processing (Kwon et al., 2010). Hence, I hypothesized that RNF157 can influence the production of A $\beta$. My experiments suggest that in the presence of Fe65, more $A \beta_{42}$ appears to be produced, which is the more toxic form among all A $\beta$ species (Klein et al., 1999; Zhang et al., 2002). However, I did not find a consistent influence of RNF157 on Fe65-mediated A $\beta$ secretion. My results also indicate that Fe65 could promote A $\beta$ production, which is in line with previous report that overexpression of Fe65 upregulates A $\beta$ levels in Madin-Darby canine kidney cells (Sabo et al., 1999). Further animal models also support the observation that deletion of Fe65 leads to reduced $A \beta_{42}$ secretion in the brain (Guenette et al., 2006). Additionally, ablation of Fe65 in mice carrying human mutant APP also suppresses $A \beta_{42}$ production (Wang et al., 2004). However, Ando and colleagues have shown that Fe65 inhibits $A \beta_{42}$ production in HEK293T (Ando et al., 2001). This discrepancy can be attributed to the different cell types. Taken together, it is more likely that Fe65 promotes A $\beta$ secretion, which in turn induces cell death. However, the link between RNF157 and Fe65mediated $\mathrm{A} \beta$ production needs to be established with further experiments.

Moreover, APP has been shown to tether Fe65 to the membrane, where Fe65 can be liberated upon phosphorylation of APP at Thr668 or Thr682 (Barbagallo et al., 2010; Nakaya and Suzuki, 2006). This adds to my hypothesis that the binding between RNF157 and Fe65 could result in the dissociation of Fe65 from APP. The interaction between RNF157 and Fe65 then facilitates the ubiquitination of Fe65 governed by RNF157. This in turn degrades Fe65, decreases its nuclear translocation and thus hinders apoptosis.

To complicate the story, a previous study revealed that tyrosine kinase c-Abl phosphorylates Fe65 at its PTB2 domain and stimulates the transcriptional activity of APP/Fe65 (Perkinton et al., 
2004). The phosphorylation of Fe65 is necessary for APP/Fe65-mediated gene transcription. Phosphorylation of substrates has been shown to have an impact on ubiquitination. For instance, the phosphorylation of c-Myc mediated by the glycogen synthase kinase 3 (GSK3) is necessary for the binding of the F-box protein Fbw7 and c-Myc, which enables the ubiquitination of c-Myc governed by the E3 ligase SCF/Fbw7 complex (Welcker et al., 2004). Accordingly, I postulate that the phosphorylation of Fe65 might be needed for its binding to RNF157 and thus facilitates the RNF157-mediated ubiquitination of Fe65, which could further affect the Fe65's turnover. To verify this assumption in RNF157/Fe65 pathway, more evidence concerning the interplay between phosphorylation and ubiquitination of Fe65 is required.

\subsection{The nuclear interactors of Fe65}

Having shown that nuclear Fe65 is as potent as the wild type Fe65 at inducing apoptsis, I reasoned that Fe65 could act with other nuclear proteins to inhibit neuronal survival. After analyzing two nuclear proteins, Tip60 and Tip110, I found that while Tip60 does not induce apoptosis, Tip110 promotes neuronal death in the RNF157/Fe65 survival pathway.

\subsubsection{Tip60 interacts with Fe65 but does not promote neuronal apoptosis}

The nuclear protein Tip60, a histone acetyltransferase (HAT), has been reported to form a complex with Fe65 and AICD, which triggers gene transcription in the nucleus (Cao and Südhof, 2001; Yamamoto and Horikoshi, 1997). Tip60 is normally localized to the nucleus; however, a small fraction can be anchored to cytoplasmic membranes by APP. This facilitates phosphorylation of Tip60 by cyclin-dependent kinase (CDK) and in turn promotes the nuclear translocation of Tip60/Fe65 to activate gene transcription (Hass and Yankner, 2005).

Much is known about the role of Tip60 in DNA damage responses and the resulting DNA repair or apoptosis (Squatrito et al., 2006). Tip60 lacking its HAT activity fails to repair doublestrand DNA breaks (Ikura et al., 2000). Upon DNA damage, the HAT coactivator TRRAP recruits Tip60 to form a complex at the DNA damage sites, which facilitates acetylation of ATM, the ataxia telangiectasia mutant kinase involved in the DNA damage response (Bakkenist and Kastan, 2003; Murr et al., 2006; Sun et al., 2005). ATM further phosphorylates p53 and triggers p53-dependent DNA repair or apoptotic pathways (Saito et al., 2002; Squatrito et al., 2006). Furthermore, Tip60 can also directly acetylate p53, which stabilizes p53 and thus induces p53- 
mediated activation of $p 21$, a cyclin-dependent kinase inhibitor, or the pro-apoptotic genes Puma and Noxa (Deng et al., 1995; Tang et al., 2006; Villunger et al., 2003). With these various options the cell can go into cell cycle arrest to attempt DNA repair, or, if not successful, initiate apoptosis. In short, these findings indicate a role of Tip60 in regulating transcription of diverse genes to regulate different events in the cell.

Intriguingly, nuclear Fe65 is required for the recruitment of Tip60-TRRAP to the double strand breaks and the resulting DNA damage response (Stante et al., 2009). Therefore, I reasoned that Tip60 could be a player in the RNF157/Fe65 survival pathway. Due to its interesting dual role in the response to DNA damage, various studies attempted to elucidate the role of Tip60 in apoptosis. Given its importance in DNA damage response, it is not surprising that knockdown of Tip60 in U2OS cells suppresses apoptosis induced by irradiation (Tyteca et al., 2006), while overexpression of Tip60 slightly increases apoptotic rate in stressed cell (Xu et al., 2009a). Furthermore, overexpression of Tip60 in unstressed SH-SY5Y cells has no effect on survival (Kim et al., 2007b). Taken together, Tip60 appears to promote cell apoptosis in heterogeneous cell systems only after DNA damage. However, a direct link between Tip60 and apoptosis in neurons is not yet well understood. Owing to its interaction with Fe65, I hypothesized that Tip60 could act together with Fe65 to regulate neuronal apoptosis.

I first verified the interaction between Fe65 and Tip60. My result supports the previous report that they do interact (Cao and Südhof, 2001). Surprisingly, my mapping analysis revealed that Tip60 interacts with the PTB2 domain of Fe65, rather than the suggested PTB1 domain (Cao and Südhof, 2001). Comparing the deletion mutants used in that study, no evidence was shown about the binding between Tip60 and the PTB2 domain. In addition, I attempted to narrow down the binding area by including further deletion mutants lacking PTB1, PTB2 or the sequence between 2 PTB domains. However, all these mutants interact with Tip60. Since mutations of a protein can alter its conformation and thus change its binding affinity with other proteins (Abrol et al., 2014), the folding structure of the PTB domains could be necessary for the interaction between Fe65 and Tip60.

I further analyzed the function of Tip60 in cerebellar granule neurons. The survival assays revealed that Tip60 by itself does not promote neuronal apoptosis. Therefore, I went on to investigate if Tip60 potentiates or attenuates Fe65-induced apoptosis. I found that overexpression of Tip60 and Fe65 does not increase the extent of apoptosis mediated by Fe65 itself. This is in line with a previous finding that Tip60 alone or Tip60 and Fe65 do not induce cell death in SHSY5Y cells (Kim et al., 2007b), although Fe65 by itself does not induce apoptosis in the same 
study. However, they found that while overexpression of AICD induces cell death, transfection of Tip60, Fe65 and AICD potentiates apoptosis up to 3-fold. Hence, it is worth considering to include AICD into further analysis. In addition, a recent study using Drosophila models demonstrated that pan-neuronal expression of mutant Tip60 that does not possess HAT activity leads to cell loss in the central nervous system, and overexpression of wild type Tip60 even worsens the apoptotic phenotype as compared to mutant Tip60 (Pirooznia et al., 2012). Interestingly, introduction of APP rescues the apoptosis induced by Tip60-overexpression in flies. This could be attributed to the observation that APP tethers Fe65 in the cytosol so that Fe65 cannot enter the nucleus to trigger apoptosis either with or without Tip60 (Nakaya et al., 2008). However, how Tip60 operates in the mammalian central nervous system needs further examination.

Furthermore, since I found that Tip60 overexpression does not worsen Fe65-triggered apoptosis, it would also be worthwhile to investigate if knockdown of Tip60 promotes or inhibits neuronal survival in the background of Fe65 overexpression. As Tip60 colocalizes with Fe65 in the nucleus (von Rotz et al., 2004), it is possible that they act as a complex to trigger apoptosis and thus the apoptotic effect of Fe65 can be alleviated upon loss of Tip60. Furthermore, Fe65 has been shown to stabilize AICD in the nucleus and form a complex with Tip60 to initiate gene transcription (Cao and Südhof, 2001; Kimberly et al., 2001; von Rotz et al., 2004). If loss of Tip60 alters the Fe65-triggered apoptotic phenotype, it can be speculated that the AICD-induced gene transcription would be blocked. Since AICD has been demonstrated to promote APP transcription as well as the accumulation of cellular APP (von Rotz et al., 2004), suppressed AICD-mediated gene expression can reduce cellular APP level. This in turn results in more nuclear translocation of Fe65 due to the lack of membrane anchoring mediated by APP, which ultimately triggers apoptosis. Another possible scenario could be that less AICD leads to a decrease of the formation of nuclear Fe65/Tip60/AICD, which in turn promotes cell survival. Furthermore, the target genes for AICD-mediated transcription are not fully identified. The verification of this model requires an extended understanding of the key molecules.

Once it is clear how Tip60 regulates apoptosis, it would be intriguing to know if RNF157 plays a role in concert with Fe65 and Tip60 to regulate neuronal survival. Since RNF157 mediates the degradation of Fe65 and loss of Fe65 could result in the dissociation of this potential apoptotic Fe65/Tip60/AICD complex and hence promote survival. Therefore, it would be worthwhile to reassess a link between RNF157 and Tip60. 


\subsubsection{Tip110, a novel interactor of Fe65, induces neuronal apoptosis}

In this study, I found that the RNA-binding protein Tip110 interacts with Fe65 and promotes neuronal apoptosis. Tip110 was first identified as a nuclear molecule that can bind RNA in vitro (Gu et al., 1998). It was later shown to directly interact with Tat, encoded by the HIV-1 regulatory gene tat, and thus involved in regulating HIV-1 gene transcription (Liu et al., 2002; Zhao et al., 2014). Tip110 is present in various human tissues with robust expression in kidney, skeletal muscle, heart and brain (Liu et al., 2002). Besides its function related to HIV-1, Tip110 has also been implicated in pre-mRNA splicing (Liu et al., 2013; Song et al., 2010), thus mediating the pluripotency of human embryonic stem cells (Liu et al., 2013). Moreover, it is involved in androgen receptor-mediated transcription (Liu et al., 2004) and hematopoiesis (Liu et al., 2011). My study reveals a novel function of Tip110 in neurons in apoptosis.

So far, no other studies propose a role for Tip110 in apoptosis. Although deletion of the Tip110 orthologue in zebrafish causes embryonic lethality (Trede et al., 2007), overexpression of human Tip110 in transgenic mice does not induce apparent developmental, behavioral or pathological alterations (Liu et al., 2011). This suggests an important role of Tip110 during early development rather than later in tissue maintenance, but interestingly, Tip110 has been shown to interact with the transcriptional factor c-Myc to operate its function in pre-mRNA processing (Liu et al., 2011; Liu et al., 2013). C-Myc is a oncoprotein, which is aberrantly upregulated in tumor cells, leading to uncontrolled cell proliferation (Pelengaris et al., 2002). Intriguingly, a transgenic model with c-Myc overexpression in the forebrain of mice supports these in vitro observations in vivo (Lee et al., 2009b). Mice with additional c-Myc develop neurodegeneration and astrocytosis in the hippocampus, which is associated with a deficit in working memory. These findings indicate the importance of c-Myc in regulating neuronal apoptosis. Since Tip110 interacts with c-Myc, it is conceivable that Tip110 promotes neuronal cell death through the cMyc-mediated apoptotic pathway. Expression of c-Myc has been shown to facilitate the release of cytochrome $\mathrm{c}$ by cooperating with the Bax-mediated mitochondrial apoptotic cascade and thus triggers apoptosis (Juin et al., 2002). Furthermore, Liu and colleagues have previously shown that while overexpression of Tip110 promotes c-Myc expression and knockdown of Tip110 reduces c-Myc level in different cell lines and human embryonic stem cells, c-Myc controls the expression of Tip110 in a similar manner (Liu et al., 2011; Liu et al., 2013). They are also shown to promote each other's transcription (Liu et al., 2011). According to this evidence, it would be interesting to explore whether c-Myc is required for Tip110 to trigger apoptosis in neurons and in addition, if Bax is a key player acting downstream of this hypothetical Tip110/c-Myc apoptotic 
pathway.

In addition, my epistasis analysis showing that Tip110 knockdown protects neurons from Fe65-mediated apoptosis suggests that Tip110 is required for Fe65 to trigger neuronal death in the nucleus. A subsequent experiment demonstrated that knockdown of Tip110 partially rescues the apoptosis induced by knockdown of RNF157, implying that other nuclear proteins could be involved in this pathway.

Taken together, my findings characterize a novel RNF157/Fe65/Tip110 pathway in the control of neuronal apoptosis. Further analyses addressing the role of other possible interactors of Fe65 or Tip110 such as Tip60 or c-Myc, respectively, could expand our understanding of the E3 ligase RNF157-mediated neuronal survival.

\subsection{The role of RNF157 in cognitive function}

Unlike mice lacking Mgrn1, which exhibit change of coat color, curly whiskers and muscular weakness, RNF157/- mice show no apparent alterations in the fur color and muscle function. This could be attributed to the brain-dominant expression of RNF157, in contrast to Mgrn1, which is present in various tissues (He et al., 2003). The behavioral analysis of RNF157 knockout mice revealed that loss of RNF157 neither affects basic motor and sensory functions nor the mice's anxious, exploratory or impulsive behaviors. Notably, intact basic functions are required for the accuracy of the memory tests.

Converging evidence indicates a role of proteasome-mediated degradation in various brain areas to regulate memory function. Inhibition of proteasomal function in hippocampus, amygdala or prefrontal cortex impairs the formation or consolidation of memory (Artinian et al., 2008; Jarome et al., 2011; Lee et al., 2008b; Reis et al., 2013). Among the components of the ubiquitin proteasome system, E3 ligases have also been reported to regulate memory function. While lack of Cdh1, the coactivator of RING-type E3 ligase APC/C, is embryonic lethal, $\mathrm{Cdh} 1^{+/}$mice exhibit deficits in fear memory ( $\mathrm{Li}$ et al., 2008a), which resembles the phenotype in heterozygous mice of SCRAPPER, an F-box protein in SCF E3 ligases (Yao et al., 2011). Furthermore, selective deletion of Cdh1 in the forebrain also showed fear memory impairment (Pick et al., 2013), whereas forebrain-specific knockout of APC2, another subunit of APC/C damages spatial memory as well as fear memory (Kuczera et al., 2011). These findings indicate an essential role of E3 ligases in cognitive function. 


\subsubsection{RNF157 has no impact on hippocampus-mediated working and spatial memory}

Due to the intriguing hippocampal cell loss in the Mgrnl null mouse and the robust expression of RNF157 in the hippocampus, I raised the question whether loss of RNF157 leads to deficits in cognitive functions. Therefore, I carried out the object recognition test and the Morris water maze test that are highly hippocampus-dependent (Broadbent et al., 2010; Garthe and Kempermann, 2013; Wang and Morris, 2010). To my surprise, I found that lack of RNF157 neither alters the formation of working memory nor affects spatial learning or memory.

Considering upregulated Fe65 levels in RNF157//- hippocampi as well as in the brain of patients with Alzheimer's disease (Delatour et al., 2001), it would be conceivable that deletion of RNF157 might lead to memory deficits. It was however not the case. This could also be attributed to the age when the test was performed. Since the accumulation of Fe65 became obvious only after 3 months of age in the RNF $157^{-/-}$brain, it could be speculated that the behavioral consequence of Fe65 upregulation would appear much later, not at 4 or even at 9 months of age when the working memory or spatial memory was assessed.

Nonetheless, my result is in line with the finding that overexpression of Fe65 in mouse does not damage working memory at 8 months of age with no neuronal loss observed in the hippocampus of these mice over 18 months old (Ghosal et al., 2009). Moreover, mice with extra human Fe65 also fail to exhibit any obvious phenotype up to 13 months of age (Santiard-Baron et al., 2005), although no behavioral data concerning these mice has been shown. On the other hand, isoform-specific deletion of 97-KDa full-length Fe65 induces impairments in hiddenplatform acquisition in Morris water maze in both young (2-4 months old) and 14-month-old mice, while no difference in hippocampal morphology can be observed at 6 months (Wang et al., 2004; Wang et al., 2009). These results suggest that Fe65 is required for hippocampal function.

Another possible explanation would be that RNF157 or Fe65 might affect memory retention but not formation. To address this hypothesis, one can examine the memory in Morris water maze much later after training. For instance, a mouse model lacking the RING-type E3 ligase cbl-b showed normal learning but enhanced long-term memory when tested 45 days after acquisition (Tan et al., 2006). If reviewing my result carefully, there seems to be a trend that $\mathrm{RNF} 157^{-/-}$mice stay longer in the target zone. It is possible that deletion of RNF157 facilitates memory retention, but this needs to be confirmed with further experiments.

Furthermore, owing to its function as an adaptor protein interacting with diverse molecules, 
the pro-apoptotic function of Fe65 might not be favored in the normal physiological environment. Instead, redundant Fe65 might damage hippocampal neurons only under certain conditions. Fe65 has been shown to induce age-dependent working memory deficits only together with AICD (Ghosal et al., 2009). The regulation of apoptosis by the Fe65 pathway in vivo might thus require other proteins that act together with Fe65 or modify its function. For instance, Fe65 also interacts with Notch1, which triggers apoptosis in neurons after ischemic stroke (Cheng et al., 2014; Kim et al., 2012). Interestingly, Fe65 has been shown to restrain Notch1 function by either facilitating its degradation or suppressing Notch1-mediated signaling (Kim et al., 2012), suggesting that Fe65 may counteract the pro-apoptotic impact of Notch1 in neurons and thus promote neuronal survival. In short, to comprehend the intact working and spatial memory in RNF157/- mice, we would need to fully assess the role of Fe65 in hippocampal function.

The other explanation for the intact hippocampal function in RNF157/- mice could be that Mgrn1 and RNF157 may be interchangeable in the brain. Mgrn1 appears to play a crucial role in neuronal health and integrity in the mouse brain as mice with a mutant Mgrnl gene develop a spongiform change in the hippocampus at 2 months of age (He et al., 2003), indicating that the presence of RNF157 cannot compensate for loss-of-Mgrn1-induced neurodegeneration. On the contrary, loss-of-RNF157 seems not to have similar outcome, hinting that Mgrn1 may have more prominent impacts and thus take over most of RNF157's functions in the RNF157/- brain. Therefore, it is important to establish if loss-of-RNF157 can lead to spongiform degeneration in vivo as seen in Mgrn1 null mice by further histological analyses of the aged RNF157/- mouse. Taken together, it could be speculated that RNF157 is essential for the neuronal integrity at the cellular level, but Mgrn1 and RNF157 could have a large degree of functional redundancy in vivo in the early phase of brain development and during young adulthood but not in the aging brain.

\subsubsection{RNF157 is required for associated fear memory}

In addition to aforementioned memory, two forms of fear memory were examined in the RNF157 transgenic mice. While the contextual fear memory requires both hippocampus and amygdala, the cued fear memory relies mainly on amygdala (Kim et al., 1993; Phillips and LeDoux, 1992). My results indicate that while RNF157//- mice seemed to have only a subtle deficit in contextual memory, deletion of RNF157 impaired the amygdala-dependent fear memory, suggesting that RNF157 is more essential for amygdala in the control of memory. The 
slight tendency in contextual condition could be attributed to the involvement of amygdala in this type of fear memory. This supports the notion that E3 ligases are essential in regulating fear memory.

Notably, patients with Alzheimer's disease exhibited deficits in fear conditioning (Hamann et al., 2002) and symptom-related amygdala as well as hippocampal atrophy (Poulin et al., 2011). This is not yet in agreement with the observations from the animal model of Alzheimer's disease. Although the APP/PS1 transgenic mice also showed impaired memory, only altered dendritic morphology but no neuronal loss in amygdala was observed (Knafo et al., 2009). The explanation could be that the dendritic phenotype contributes to the fear memory deficits and a general imbalance in the central nervous system. On the contrary, an imbalanced central nervous system could be the cause for the change in dendrites. In both scenarios, the final consequence of a disturbed cell signaling could be the cell death seen in patients. Since it has been shown that RNF157 can also promote dendrite growth independent of its ligase activity (Matz et al., in press), it could be the reason for the impaired memory when RNF157 is absent in amygdala. The molecular pathway underlying RNF157-mediated dendrite development however needs further research.

The possibility that loss of RNF157 can lead to neuronal apoptosis in amygdala can still not be excluded. Therefore, it is worthwhile to examine, first of all, the expression pattern of RNF157 and Fe65 in the amygdala. Secondly, if neurons in amygdala develop shorter dendrites or are more prone to apoptosis in RNF157/- mice should be addressed. If the RNF157/Fe65 survival pathway is also active in the amygdala, I speculate that Fe65 could mediate the expression of genes that govern fear memory formation during fear conditioning since Fe65 is involved in the transcription of various genes (Minopoli et al., 2012). Hence, it would be interesting to identify the target genes of Fe65 during fear conditioning and if Tip60, Tip110 or other proteins operate together with Fe65 to regulate these genes.

Additionally, the study using Fe65 $5^{-/-}$mice demonstrated that deletion of Fe65 impaired both hippocampal-dependent contextual fear memory and the induction of associated long-term potentiation (LTP) in the hippocampus (Wang et al., 2009). However, no data concerning amygdala function has been shown in this mouse model. Owing to its strong association with cued fear memory, the long-term potentiation (LTP) in amygdala is worth being examined (Rogan et al., 1997). I would expect defective LTP in the RNF157/- amygdala. Furthermore, it would be interesting to examine LTP in hippocampus since a trend of impaired contextual memory can be seen in mice lacking RNF157. This could address if Fe65 has a different function 
in hippocampus and amygdala or if RNF157 acts in distinct ways in various brain regions. Lastly, due to its ability to bind to Fe65 (Matz et al., in press), the function of Mgrn1 in these brain areas should be taken into consideration. Whether Mgrn1 compensates the function of RNF157 in these parts of the brain would induce different outcome of RNF157 knockdown. If Mgrn1 is the functionally redundant homologue of RNF157 in neurons, a Mgrn1/RNF157 double knockout mouse model would help to elaborate the real impact of RNF157 in the central nervous system.

\subsection{Conclusion}

My study extends our understanding of the brain-dominant E3 ligase RNF157 in the control of neuronal survival. Here I show that the novel interactor of RNF157, the adaptor protein Fe65 is pro-apoptotic and operates downstream of RNF157 in the control of neuronal apoptosis. Importantly, RNF157 counteracts Fe65's pro-apoptotic function by attaching the K63-linked polyubiquitin chain on Fe65, which atypically targets Fe65 for proteasomal degradation and thus protects neurons from cell death. In hippocampal neurons, Fe65 acts mainly in the nucleus to trigger apoptosis. I further elucidated this pathway and identified a novel interactor of nuclear Fe65, the nuclear RNA-binding protein Tip110. Like Fe65, Tip110 induces neuronal apoptosis. Further epistasis analysis demonstrated that Tip110 is required for Fe65 to trigger cell death. In short, my in vitro work uncovers a novel RNF157/Fe65/Tip110 pathway in the control of neuronal survival. Based on these and other findings, I propose a working model depicted in Fig 4.1 .

In addition, the behavioral analyses of RNF157//- mice indicate that loss of RNF157 does not induce a severe pathological outcome in mouse behavior at young age. Notably, I found that deletion of rnf157 impairs the amygdala-mediated fear memory. My study elaborates on the role of RNF157 in neuronal survival as well as in fear memory.

In conclusion, my work provides further insight into the pro-survival function of the E3 ligase RNF157 and its role in cognitive function. Further research is necessary to establish a link between RNF157 and neurodegeneration in vivo that could ultimately contribute to deeper understanding of ubiquitination in neurodegenerative diseases. 


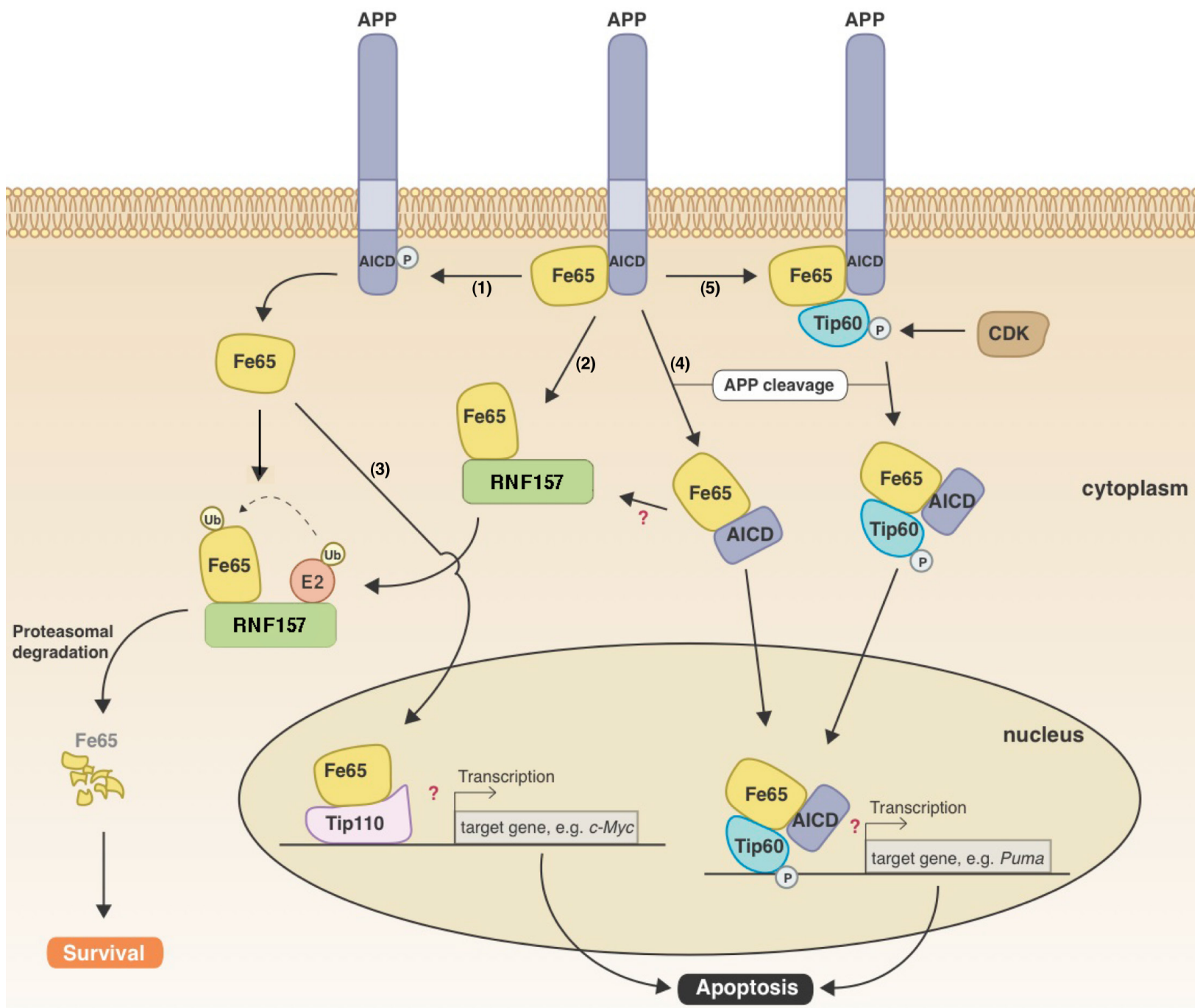

Fig 4.1 Current working model of the E3 ligase RNF157 in neuronal survival.

The adaptor protein Fe65, can be tethered by APP to the membrane upon their interaction (Nakaya and Suzuki, 2006). (1) When APP is phosphorylated, Fe65 is liberated from APP into the cytoplasm (Nakaya and Suzuki, 2006). RNF157 can therefore bind to Fe65 and ubiquitinate it. (2) Alternatively, RNF157 can directly compete with APP for the binding to Fe65. In both scenarios, Fe65 is targeted for proteasomal degradation. (3) In the absence of RNF157, cytoplasmic Fe65 can translocate into the nucleus and act together with Tip110 to trigger the transcription of its target genes resulting in apoptosis. (4) Fe65 can also translocate into the nucleus together with AICD to trigger gene transcription, which may lead to cell death (Cao and Südhof, 2001; Perkinton et al., 2004; von Rotz et al., 2004) or (5) initiate the putative apoptotic pathway mediated by AICD, Fe65 and Tip60. Briefly, cyclindependent kinase (CDK) activates Tip60 by phosphorylation (Hass and Yankner, 2005). Together with activated Tip60, Fe65 and AICD can form a trimeric complex, which translocates either together or separately in the nucleus to stimulate gene transcription (Cao and Sudhof, 2004; Cao and Südhof, 2001). However, several questions remains unsolved in the RNF157-mediated survival mechanism: i) can RNF157 also compete with AICD for the binding to Fe65 to inhibit the pathway (4), ii) is Tip60 required for Fe65 to induce apoptosis, and iii) which are the target genes in the Tip110 or Tip60-mediated gene expression? 


\section{References}

Abrol, R., Trzaskowski, B., Goddard, W.A., 3rd, Nesterov, A., Olave, I., and Irons, C. (2014). Ligand- and mutation-induced conformational selection in the CCR5 chemokine G proteincoupled receptor. Proc Natl Acad Sci U S A 111, 13040-13045.

Akao, Y., Otsuki, Y., Kataoka, S., Ito, Y., and Tsujimoto, Y. (1994). Multiple SubcellularLocalization of Bcl-2 - Detection in Nuclear Outer-Membrane, Endoplasmic-Reticulum Membrane, and Mitochondrial-Membranes. Cancer Res 54, 2468-2471.

Almeida, A., Bolanos, J.P., and Moreno, S. (2005). Cdh1/Hct1-APC is essential for the survival of postmitotic neurons. J Neurosci 25, 8115-8121.

Andalib, S., Vafaee, M.S., and Gjedde, A. (2014). Parkinson's disease and mitochondrial gene variations: A review. J Neurol Sci.

Ando, K., Iijima, K.I., Elliott, J.I., Kirino, Y., and Suzuki, T. (2001). Phosphorylation-dependent regulation of the interaction of amyloid precursor protein with Fe65 affects the production of beta-amyloid. J Biol Chem 276, 40353-40361.

Aravind, L., and Koonin, E.V. (2000). The U box is a modified RING finger - a common domain in ubiquitination. Current Biology 10, R132-R134.

Artinian, J., McGauran, A.M., De Jaeger, X., Mouledous, L., Frances, B., and Roullet, P. (2008). Protein degradation, as with protein synthesis, is required during not only long-term spatial memory consolidation but also reconsolidation. Eur J Neurosci 27, 3009-3019.

Ashkenazi, A., and Dixit, V.M. (1998). Death receptors: signaling and modulation. Science 281, 1305-1308.

Atabay, C., Cagnoli, C.M., Kharlamov, E., Ikonomovic, M.D., and Manev, H. (1996). Removal of serum from primary cultures of cerebellar granule neurons induces oxidative stress and DNA fragmentation: protection with antioxidants and glutamate receptor antagonists. J Neurosci Res 43, 465-475.

Atkin, G., Hunt, J., Minakawa, E., Sharkey, L., Tipper, N., Tennant, W., and Paulson, H.L. (2014) F-box Only Protein 2 (Fbxo2) Regulates Amyloid Precursor Protein Levels and Processing. Journal of Biological Chemistry 289, 7038-7048.

Atkin, G., and Paulson, H. (2014). Ubiquitin pathways in neurodegenerative disease. Front Mol Neurosci 7, 63.

Bakkenist, C.J., and Kastan, M.B. (2003). DNA damage activates ATM through intermolecular autophosphorylation and dimer dissociation. Nature 421, 499-506.

Barbagallo, A.P., Weldon, R., Tamayev, R., Zhou, D., Giliberto, L., Foreman, O., and D'Adamio, L. (2010). $\operatorname{Tyr}(682)$ in the intracellular domain of APP regulates amyloidogenic APP processing in vivo. PLoS One 5, e15503.

Barbato, C., Canu, N., Zambrano, N., Serafino, A., Minopoli, G., Ciotti, M.T., Amadoro, G., Russo, T., and Calissano, P. (2005). Interaction of Tau with Fe65 links tau to APP. Neurobiol Dis 18, 399-408.

Becher, M.W., Kotzuk, J.A., Sharp, A.H., Davies, S.W., Bates, G.P., Price, D.L., and Ross, C.A. (1998). Intranuclear neuronal inclusions in Huntington's disease and dentatorubral and pallidoluysian atrophy: correlation between the density of inclusions and IT15 CAG triplet repeat length. Neurobiol Dis 4, 387-397. 
Becker, E.B., and Bonni, A. (2004). Cell cycle regulation of neuronal apoptosis in development and disease. Prog Neurobiol 72, 1-25.

Bedford, L., Layfield, R., Mayer, R.J., Peng, J., and Xu, P. (2011). Diverse polyubiquitin chains accumulate following $26 \mathrm{~S}$ proteasomal dysfunction in mammalian neurones. Neurosci Lett 491, 44-47.

Berndsen, C.E., and Wolberger, C. (2014). New insights into ubiquitin E3 ligase mechanism. Nat Struct Mol Biol 21, 301-307.

Billen, L.P., Kokoski, C.L., Lovell, J.F., Leber, B., and Andrews, D.W. (2008). Bcl-XL inhibits membrane permeabilization by competing with Bax. Plos Biol 6, 1268-1280.

Bodda, C., Tantra, M., Mollajew, R., Arunachalam, J.P., Laccone, F.A., Can, K., Rosenberger, A., Mironov, S.L., Ehrenreich, H., and Mannan, A.U. (2013). Mild overexpression of Mecp2 in mice causes a higher susceptibility toward seizures. Am J Pathol 183, 195-210.

Borg, J.P., Ooi, J., Levy, E., and Margolis, B. (1996). The phosphotyrosine interaction domains of X11 and FE65 bind to distinct sites on the YENPTY motif of amyloid precursor protein. Molecular and Cellular Biology 16, 6229-6241.

Borquez, D.A., and Gonzalez-Billault, C. (2012). The amyloid precursor protein intracellular domain-fe65 multiprotein complexes: a challenge to the amyloid hypothesis for Alzheimer's disease? Int J Alzheimers Dis 2012, 353145.

Broadbent, N.J., Gaskin, S., Squire, L.R., and Clark, R.E. (2010). Object recognition memory and the rodent hippocampus. Learn Mem 17, 5-11.

Brouillette, J., Caillierez, R., Zommer, N., Alves-Pires, C., Benilova, I., Blum, D., De Strooper, B., and Buee, L. (2012). Neurotoxicity and memory deficits induced by soluble lowmolecular-weight amyloid-beta1-42 oligomers are revealed in vivo by using a novel animal model. J Neurosci 32, 7852-7861.

Brunet, A., Datta, S.R., and Greenberg, M.E. (2001). Transcription-dependent and -independent control of neuronal survival by the PI3K-Akt signaling pathway. Curr Opin Neurobiol 11, 297-305.

Bruni, P., Minopoli, G., Brancaccio, T., Napolitano, M., Faraonio, R., Zambrano, N., Hansen, U., and Russo, T. (2002). Fe65, a ligand of the Alzheimer's beta-amyloid precursor protein, blocks cell cycle progression by down-regulating thymidylate synthase expression. J Biol Chem 277, 35481-35488.

Bryan, B., Cai, Y., Wrighton, K., Wu, G., Feng, X.H., and Liu, M. (2005). Ubiquitination of RhoA by Smurf1 promotes neurite outgrowth. Febs Lett 579, 1015-1019.

Cao, X., and Sudhof, T.C. (2004). Dissection of amyloid-beta precursor protein-dependent transcriptional transactivation. J Biol Chem 279, 24601-24611.

Cao, X., and Südhof, T.C. (2001). A transcriptionally [correction of transcriptively] active complex of APP with Fe65 and histone acetyltransferase Tip60. Science 293, 115-120.

Certo, M., Del Gaizo Moore, V., Nishino, M., Wei, G., Korsmeyer, S., Armstrong, S.A., and Letai, A. (2006). Mitochondria primed by death signals determine cellular addiction to antiapoptotic BCL-2 family members. Cancer Cell 9, 351-365.

Cheng, E.H.Y.A., Wei, M.C., Weiler, S., Flavell, R.A., Mak, T.W., Lindsten, T., and Korsmeyer, S.J. (2001). BCL-2, BCL-X-L sequester BH3 domain-only molecules preventing BAX- and BAK-mediated mitochondrial apoptosis. Molecular Cell 8, 705-711.

Cheng, Y.L., Park, J.S., Manzanero, S., Choi, Y., Baik, S.H., Okun, E., Gelderblom, M., Fann, D.Y.W., Magnus, T., Launikonis, B.S., et al. (2014). Evidence that collaboration between HIF-1 alpha and Notch-1 promotes neuronal cell death in ischemic stroke. Neurobiol Dis 62, 
286-295.

Chhangani, D., and Mishra, A. (2013). Mahogunin ring finger-1 (MGRN1) suppresses chaperone-associated misfolded protein aggregation and toxicity. Sci Rep 3, 1972.

Chipuk, J.E., and Green, D.R. (2005). Do inducers of apoptosis trigger caspase-independent cell death? Nat Rev Mol Cell Bio 6, 268-275.

Chow, W.N., Luk, H.W., Chan, H.Y., and Lau, K.F. (2012). Degradation of mutant huntingtin via the ubiquitin/proteasome system is modulated by FE65. Biochem J 443, 681-689.

Chung, C.W., Song, Y.H., Kim, I.K., Yoon, W.J., Ryu, B.R., Jo, D.G., Woo, H.N., Kwon, Y.K., Kim, H.H., Gwag, B.J., et al. (2001). Proapoptotic effects of Tau cleavage product generated by caspase-3. Neurobiol Dis $8,162-172$.

Ciechanover, A. (2013). Intracellular protein degradation: From a vague idea through the lysosome and the ubiquitin-proteasome system and onto human diseases and drug targeting. Bioorgan Med Chem 21, 3400-3410.

Ciechanover, A., and Brundin, P. (2003). The ubiquitin proteasome system in neurodegenerative diseases: sometimes the chicken, sometimes the egg. Neuron 40, 427-446.

Citron, M., Oltersdorf, T., Haass, C., McConlogue, L., Hung, A.Y., Seubert, P., Vigo-Pelfrey, C., Lieberburg, I., and Selkoe, D.J. (1992). Mutation of the beta-amyloid precursor protein in familial Alzheimer's disease increases beta-protein production. Nature 360, 672-674.

Citron, M., Vigo-Pelfrey, C., Teplow, D.B., Miller, C., Schenk, D., Johnston, J., Winblad, B., Venizelos, N., Lannfelt, L., and Selkoe, D.J. (1994). Excessive production of amyloid betaprotein by peripheral cells of symptomatic and presymptomatic patients carrying the Swedish familial Alzheimer disease mutation. Proc Natl Acad Sci U S A 91, 11993-11997.

Cregan, S.P., Fortin, A., MacLaurin, J.G., Callaghan, S.M., Cecconi, F., Yu, S.W., Dawson, T.M., Dawson, V.L., Park, D.S., Kroemer, G., et al. (2002). Apoptosis-inducing factor is involved in the regulation of caspase-independent neuronal cell death. J Cell Biol 158, 507-517.

Culmsee, C., and Mattson, M.P. (2005). p53 in neuronal apoptosis. Biochem Biophys Res Commun 331, 761-777.

D'Mello, S.R., Galli, C., Ciotti, T., and Calissano, P. (1993). Induction of apoptosis in cerebellar granule neurons by low potassium: inhibition of death by insulin-like growth factor I and cAMP. Proc Natl Acad Sci U S A 90, 10989-10993.

Dantuma, N.P., and Bott, L.C. (2014). The ubiquitin-proteasome system in neurodegenerative diseases: precipitating factor, yet part of the solution. Front Mol Neurosci 7, 70.

Datta, S.R., Brunet, A., and Greenberg, M.E. (1999). Cellular survival: a play in three Akts. Gene Dev 13, 2905-2927.

Datta, S.R., Dudek, H., Tao, X., Masters, S., Fu, H.A., Gotoh, Y., and Greenberg, M.E. (1997). Akt phosphorylation of BAD couples survival signals to the cell-intrinsic death machinery. Cell 91, 231-241.

Delatour, B., Mercken, L., El Hachimi, K.H., Colle, M.A., Pradier, L., and Duyckaerts, C. (2001). FE65 in Alzheimer's disease: neuronal distribution and association with neurofibrillary tangles. Am J Pathol 158, 1585-1591.

Deng, C., Zhang, P., Harper, J.W., Elledge, S.J., and Leder, P. (1995). Mice lacking p21CIP1/WAF1 undergo normal development, but are defective in G1 checkpoint control. Cell 82, 675-684.

Desagher, S., Osen-Sand, A., Nichols, A., Eskes, R., Montessuit, S., Lauper, S., Maundrell, K., Antonsson, B., and Martinou, J.C. (1999). Bid-induced conformational change of Bax is responsible for mitochondrial cytochrome c release during apoptosis. Journal of Cell 
Biology 144, 891-901.

Deshaies, R.J., and Joazeiro, C.A. (2009). RING domain E3 ubiquitin ligases. Annu Rev Biochem 78, 399-434.

Deveraux, Q.L., Leo, E., Stennicke, H.R., Welsh, K., Salvesen, G.S., and Reed, J.C. (1999). Cleavage of human inhibitor of apoptosis protein XIAP results in fragments with distinct specificities for caspases. Embo Journal 18, 5242-5251.

DiFiglia, M., Sapp, E., Chase, K.O., Davies, S.W., Bates, G.P., Vonsattel, J.P., and Aronin, N. (1997). Aggregation of huntingtin in neuronal intranuclear inclusions and dystrophic neurites in brain. Science 277, 1990-1993.

Du, C.Y., Fang, M., Li, Y.C., Li, L., and Wang, X.D. (2000). Smac, a mitochondrial protein that promotes cytochrome c-dependent caspase activation by eliminating IAP inhibition. Cell $102,33-42$.

Durrieu, F., Samejima, K., Fortune, J.M., Kandels-Lewis, S., Osheroff, N., and Earnshaw, W.C. (2000). DNA topoisomerase II alpha interacts with CAD nuclease and is involved in chromatin condensation during apoptotic execution. Current Biology 10, 923-926.

Edlich, F., Banerjee, S., Suzuki, M., Cleland, M.M., Arnoult, D., Wang, C.X., Neutzner, A., Tjandra, N., and Youle, R.J. (2011). Bcl-x(L) Retrotranslocates Bax from the Mitochondria into the Cytosol. Cell 145, 104-116.

Elmore, S. (2007). Apoptosis: a review of programmed cell death. Toxicol Pathol 35, 495-516.

Enari, M., Sakahira, H., Yokoyama, H., Okawa, K., Iwamatsu, A., and Nagata, S. (1998). A caspase-activated DNase that degrades DNA during apoptosis, and its inhibitor ICAD. Nature 391, 43-50.

Ennaceur, A., and Delacour, J. (1988). A New One-Trial Test for Neurobiological Studies of Memory in Rats .1. Behavioral-Data. Behav Brain Res 31, 47-59.

Ermekova, K.S., Zambrano, N., Linn, H., Minopoli, G., Gertler, F., Russo, T., and Sudol, M. (1997). The WW domain of neural protein FE65 interacts with proline-rich motifs in Mena, the mammalian homolog of Drosophila enabled. Journal of Biological Chemistry 272, 32869-32877.

Erwig, L.P., and Henson, P.M. (2008). Clearance of apoptotic cells by phagocytes. Cell Death and Differentiation 15, 243-250.

Fang, S.Y., Jensen, J.P., Ludwig, R.L., Vousden, K.H., and Weissman, A.M. (2000). Mdm2 is a RING finger-dependent ubiquitin protein ligase for itself and p53. Journal of Biological Chemistry 275, 8945-8951.

Fiore, F., Zambrano, N., Minopoli, G., Donini, V., Duilio, A., and Russo, T. (1995). The regions of the Fe65 protein homologous to the phosphotyrosine interaction/phosphotyrosine binding domain of Shc bind the intracellular domain of the Alzheimer's amyloid precursor protein. $\mathrm{J}$ Biol Chem 270, 30853-30856.

Fox, M.W. (1965). The visual cliff test for the study of visual depth perception in the mouse. Anim Behav 13, 232-233.

Furukawa, M., He, Y.J., Borchers, C., and Xiong, Y. (2003). Targeting of protein ubiquitination by BTB-Cullin 3-Roc1 ubiquitin ligases. Nat Cell Biol 5, 1001-1007.

Garthe, A., and Kempermann, G. (2013). An old test for new neurons: refining the Morris water maze to study the functional relevance of adult hippocampal neurogenesis. Front Neurosci 7 , 63.

Geetha, T., Kenchappa, R.S., Wooten, M.W., and Carter, B.D. (2005). TRAF6-mediated ubiquitination regulates nuclear translocation of NRIF, the p75 receptor interactor. EMBO J 
24, 3859-3868.

Ghosal, K., Vogt, D.L., Liang, M., Shen, Y., Lamb, B.T., and Pimplikar, S.W. (2009). Alzheimer's disease-like pathological features in transgenic mice expressing the APP intracellular domain. Proc Natl Acad Sci U S A 106, 18367-18372.

Gorin, P.D., and Johnson, E.M. (1980). Effects of Long-Term Nerve Growth-Factor Deprivation on the Nervous-System of the Adult-Rat - an Experimental Autoimmune Approach. Brain Res 198, 27-42.

Götz, J., and Ittner, L.M. (2008). Animal models of Alzheimer's disease and frontotemporal dementia. Nat Rev Neurosci 9, 532-544.

Goulart, B.K., de Lima, M.N.M., de Farias, C.B., Reolon, G.K., Almeida, V.R., Quevedo, J., Kapczinski, F., Schroder, N., and Roesler, R. (2010). Ketamine Impairs Recognition Memory Consolidation and Prevents Learning-Induced Increase in Hippocampal BrainDerived Neurotrophic Factor Levels. Neuroscience 167, 969-973.

Gregory, G.C., and Halliday, G.M. (2005). What is the dominant Abeta species in human brain tissue? A review. Neurotox Res 7, 29-41.

Gross, A., Jockel, J., Wei, M.C., and Korsmeyer, S.J. (1998). Enforced dimerization of BAX results in its translocation, mitochondrial dysfunction and apoptosis. Embo Journal 17, 3878-3885.

Gu, J., Shimba, S., Nomura, N., and Reddy, R. (1998). Isolation and characterization of a new $110 \mathrm{kDa}$ human nuclear RNA-binding protein (p110nrb). Biochim Biophys Acta 1399, 1-9.

Guenette, S., Chang, Y., Hiesberger, T., Richardson, J.A., Eckman, C.B., Eckman, E.A., Hammer, R.E., and Herz, J. (2006). Essential roles for the FE65 amyloid precursor protein-interacting proteins in brain development. EMBO J 25, 420-431.

Guenette, S.Y., Chen, J., Jondro, P.D., and Tanzi, R.E. (1996a). Association of a novel human FE65-like protein with the cytoplasmic domain of the beta-amyloid precursor protein. Proc Natl Acad Sci U S A 93, 10832-10837.

Guenette, S.Y., Chen, J., Jondro, P.D., and Tanzi, R.E. (1996b). Association of a novel human FE65-like protein with the cytoplasmic domain of the beta-amyloid precursor protein. P Natl Acad Sci USA 93, 10832-10837.

Hamann, S., Monarch, E.S., and Goldstein, F.C. (2002). Impaired fear conditioning in Alzheimer's disease. Neuropsychologia 40, 1187-1195.

Hammond, R.S., Tull, L.E., and Stackman, R.W. (2004). On the delay-dependent involvement of the hippocampus in object recognition memory. Neurobiol Learn Mem 82, 26-34.

Hardy, J., and Selkoe, D.J. (2002). Medicine - The amyloid hypothesis of Alzheimer's disease: Progress and problems on the road to therapeutics. Science 297, 353-356.

Hashimoto, Y., Jiang, H., Niikura, T., Ito, Y., Hagiwara, A., Umezawa, K., Abe, Y., Murayama, Y., and Nishimoto, I. (2000). Neuronal apoptosis by apolipoprotein E4 through low-density lipoprotein receptor-related protein and heterotrimeric GTPases. J Neurosci 20, 8401-8409.

Hass, M.R., and Yankner, B.A. (2005). A \{gamma\}-secretase-independent mechanism of signal transduction by the amyloid precursor protein. J Biol Chem 280, 36895-36904.

Haupt, Y., Maya, R., Kazaz, A., and Oren, M. (1997). Mdm2 promotes the rapid degradation of p53. Nature 387, 296-299.

He, L., Lu, X.Y., Jolly, A.F., Eldridge, A.G., Watson, S.J., Jackson, P.K., Barsh, G.S., and Gunn, T.M. (2003). Spongiform degeneration in mahoganoid mutant mice. Science 299, 710-712.

Hegde, R., Srinivasula, S.M., Zhang, Z.J., Wassell, R., Mukattash, R., Cilenti, L., DuBois, G., Lazebnik, Y., Zervos, A.S., Fernandes-Alnemri, T., et al. (2002). Identification of Omi/HtrA- 
2 as a mitochondrial apoptotic serine protease that disrupts inhibitor of apoptosis proteincaspase interaction. Journal of Biological Chemistry 277, 432-438.

Hemmings, B.A., and Restuccia, D.F. (2012). Pi3k-Pkb/Akt Pathway. Csh Perspect Biol 4.

Hoe, H.S., Magill, L.A., Guenette, S., Fu, Z.Y., Vicini, S., and Rebeck, G.W. (2006). FE65 interaction with the ApoE receptor ApoEr2. Journal of Biological Chemistry 281, 2452124530.

Hoe, H.S., Wessner, D., Beffert, U., Becker, A.G., Matsuoka, Y., and Rebeck, G.W. (2005). Fspondin interaction with the apolipoprotein E receptor ApoEr2 affects processing of amyloid precursor protein. Molecular and Cellular Biology 25, 9259-9268.

Hsu, Y.T., Wolter, K.G., and Youle, R.J. (1997). Cytosol-to-membrane redistribution of Bax and Bcl-X(L) during apoptosis. Proc Natl Acad Sci U S A 94, 3668-3672.

Hu, S.M., and Yang, X.L. (2003). Cellular inhibitor of apoptosis 1 and 2 are ubiquitin ligases for the apoptosis inducer Smac/DIABLO. Journal of Biological Chemistry 278, 10055-10060.

Huntwork-Rodriguez, S., Wang, B., Watkins, T., Ghosh, A.S., Pozniak, C.D., Bustos, D., Newton, K., Kirkpatrick, D.S., and Lewcock, J.W. (2013). JNK-mediated phosphorylation of DLK suppresses its ubiquitination to promote neuronal apoptosis. J Cell Biol 202, 747-763.

Idan-Feldman, A., Ostritsky, R., and Gozes, I. (2012). Tau and caspase 3 as targets for neuroprotection. Int J Alzheimers Dis 2012, 493670.

Ikura, T., Ogryzko, V.V., Grigoriev, M., Groisman, R., Wang, J., Horikoshi, M., Scully, R., Qin, J., and Nakatani, Y. (2000). Involvement of the TIP60 histone acetylase complex in DNA repair and apoptosis. Cell 102, 463-473.

Jarome, T.J., Werner, C.T., Kwapis, J.L., and Helmstetter, F.J. (2011). Activity Dependent Protein Degradation Is Critical for the Formation and Stability of Fear Memory in the Amygdala. PLoS One 6.

Jesenberger, V., and Jentsch, S. (2002). Deadly encounter: ubiquitin meets apoptosis. Nat Rev Mol Cell Biol 3, 112-121.

Jiao, J., Sun, K.H., Walker, W.P., Bagher, P., Cota, C.D., and Gunn, T.M. (2009). Abnormal regulation of TSG101 in mice with spongiform neurodegeneration. Bba-Mol Basis Dis 1792, 1027-1035.

Johnson, B.N., Berger, A.K., Cortese, G.P., and Lavoie, M.J. (2012). The ubiquitin E3 ligase parkin regulates the proapoptotic function of Bax. Proc Natl Acad Sci U S A 109, 6283-6288.

Jordan, J., Galindo, M.F., Prehn, J.H.M., Weichselbaum, R.R., Beckett, M., Ghadge, G.D., Roos, R.P., Leiden, J.M., and Miller, R.J. (1997). P53 expression induces apoptosis in hippocampal pyramidal neuron cultures. J Neurosci 17, 1397-1405.

Joza, N., Susin, S.A., Daugas, E., Stanford, W.L., Cho, S.K., Li, C.Y., Sasaki, T., Elia, A.J., Cheng, H.Y., Ravagnan, L., et al. (2001). Essential role of the mitochondrial apoptosisinducing factor in programmed cell death. Nature 410, 549-554.

Juin, P., Hunt, A., Littlewood, T., Griffiths, B., Swigart, L.B., Korsmeyer, S., and Evan, G. (2002). c-Myc functionally cooperates with Bax to induce apoptosis. Mol Cell Biol 22, 6158-6169.

Kamine, J., Elangovan, B., Subramanian, T., Coleman, D., and Chinnadurai, G. (1996). Identification of a cellular protein that specifically interacts with the essential cysteine region of the HIV-1 Tat transactivator. Virology 216, 357-366.

Kaneko, M., Koike, H., Saito, R., Kitamura, Y., Okuma, Y., and Nomura, Y. (2010). Loss of HRD1-Mediated Protein Degradation Causes Amyloid Precursor Protein Accumulation and Amyloid-beta Generation. J Neurosci 30, 3924-3932.

Kapuria, V., Peterson, L.F., Fang, D., Bornmann, W.G., Talpaz, M., and Donato, N.J. (2010). 
Deubiquitinase Inhibition by Small-Molecule WP1130 Triggers Aggresome Formation and Tumor Cell Apoptosis. Cancer Res 70, 9265-9276.

Kaufmann, T., Schlipf, S., Sanz, J., Neubert, K., Stein, R., and Borner, C. (2003). Characterization of the signal that directs $\mathrm{Bcl}-\mathrm{x}(\mathrm{L})$, but not $\mathrm{Bcl}-2$, to the mitochondrial outer membrane. Journal of Cell Biology 160, 53-64.

Kawabe, H., and Brose, N. (2011). The role of ubiquitylation in nerve cell development. Nat Rev Neurosci 12, 251-268.

Kawabe, H., Neeb, A., Dimova, K., Young, S.M., Jr., Takeda, M., Katsurabayashi, S., Mitkovski, M., Malakhova, O.A., Zhang, D.E., Umikawa, M., et al. (2010). Regulation of Rap2A by the ubiquitin ligase Nedd4-1 controls neurite development. Neuron 65, 358-372.

Kim, B.Y., Olzmann, J.A., Barsh, G.S., Chin, L.S., and Li, L. (2007a). Spongiform neurodegeneration-associated E3 ligase Mahogunin ubiquitylates TSG101 and regulates endosomal trafficking. Mol Biol Cell 18, 1129-1142.

Kim, H., Rafiuddin-Shah, M., Tu, H.C., Jeffers, J.R., Zambetti, G.P., Hsieh, J.J., and Cheng, E.H. (2006). Hierarchical regulation of mitochondrion-dependent apoptosis by BCL-2 subfamilies. Nat Cell Biol 8, 1348-1358.

Kim, J.J., Rison, R.A., and Fanselow, M.S. (1993). Effects of amygdala, hippocampus, and periaqueductal gray lesions on short- and long-term contextual fear. Behav Neurosci 107, 1093-1098.

Kim, M.Y., Mo, J.S., Ann, E.J., Yoon, J.H., and Park, H.S. (2012). Dual regulation of notch1 signaling pathway by adaptor protein fe65. J Biol Chem 287, 4690-4701.

Kim, S.Y., Kim, M.Y., Mo, J.S., and Park, H.S. (2007b). Notch1 intracellular domain suppresses APP intracellular domain-Tip60-Fe65 complex mediated signaling through physical interaction. Biochim Biophys Acta 1773, 736-746.

Kimberly, W.T., Zheng, J.B., Guenette, S.Y., and Selkoe, D.J. (2001). The intracellular domain of the beta-amyloid precursor protein is stabilized by Fe65 and translocates to the nucleus in a Notch-like manner. Journal of Biological Chemistry 276, 40288-40292.

Kimura, A., and Horikoshi, M. (1998). Tip60 acetylates six lysines of a specific class in core histones in vitro. Genes Cells 3, 789-800.

Kitada, T., Asakawa, S., Hattori, N., Matsumine, H., Yamamura, Y., Minoshima, S., Yokochi, M., Mizuno, Y., and Shimizu, N. (1998). Mutations in the parkin gene cause autosomal recessive juvenile parkinsonism. Nature 392, 605-608.

Klein, A.M., Kowall, N.W., and Ferrante, R.J. (1999). Neurotoxicity and oxidative damage of beta amyloid 1-42 versus beta amyloid 1-40 in the mouse cerebral cortex. Ann N Y Acad Sci 893, 314-320.

Knafo, S., Venero, C., Merino-Serrais, P., Fernaud-Espinosa, I., Gonzalez-Soriano, J., Ferrer, I., Santpere, G., and DeFelipe, J. (2009). Morphological alterations to neurons of the amygdala and impaired fear conditioning in a transgenic mouse model of Alzheimer's disease. J Pathol $219,41-51$.

Kolb, B., Sutherland, R.J., and Whishaw, I.Q. (1983). A Comparison of the Contributions of the Frontal and Parietal Association Cortex to Spatial Localization in Rats. Behavioral Neuroscience 97, 13-27.

Komander, D., and Rape, M. (2012). The Ubiquitin Code. Annu Rev Biochem 81, 203-229.

Korolchuk, V.I., Menzies, F.M., and Rubinsztein, D.C. (2010). Mechanisms of cross-talk between the ubiquitin-proteasome and autophagy-lysosome systems. Febs Lett 584, 13931398. 
Kriem, B., Sponne, I., Fifre, A., Malaplate-Armand, C., Lozac'h-Pillot, K., Koziel, V., Yen-Potin, F.T., Bihain, B., Oster, T., Olivier, J.L., et al. (2005). Cytosolic phospholipase A2 mediates neuronal apoptosis induced by soluble oligomers of the amyloid-beta peptide. Faseb J 19, 85-87.

Kruman, II, Wersto, R.P., Cardozo-Pelaez, F., Smilenov, L., Chan, S.L., Chrest, F.J., Emokpae, R., Jr., Gorospe, M., and Mattson, M.P. (2004). Cell cycle activation linked to neuronal cell death initiated by DNA damage. Neuron 41, 549-561.

Kuan, C.Y., Roth, K.A., Flavell, R.A., and Rakic, P. (2000). Mechanisms of programmed cell death in the developing brain. Trends Neurosci 23, 291-297.

Kuczera, T., Stilling, R.M., Hsia, H.E., Bahari-Javan, S., Irniger, S., Nasmyth, K., Sananbenesi, F., and Fischer, A. (2011). The anaphase promoting complex is required for memory function in mice. Learn Memory 18, 49-57.

Kuhlenbaumer, G., Young, P., Oberwittler, C., Hunermund, G., Schirmacher, A., Domschke, K., Ringelstein, B., and Stogbauer, F. (2002). Giant axonal neuropathy (GAN): case report and two novel mutations in the gigaxonin gene. Neurology 58, 1273-1276.

Kuwana, T., Bouchier-Hayes, L., Chipuk, J.E., Bonzon, C., Sullivan, B.A., Green, D.R., and Newmeyer, D.D. (2005). BH3 domains of BH3-only proteins differentially regulate Baxmediated mitochondrial membrane permeabilization both directly and indirectly. Mol Cell 17, 525-535.

Kvansakul, M., Yang, H., Fairlie, W.D., Czabotar, P.E., Fischer, S.F., Perugini, M.A., Huang, D.C.S., and Colman, P.M. (2008). Vaccinia virus anti-apoptotic F1L is a novel Bcl-2-like domain-swapped dimer that binds a highly selective subset of $\mathrm{BH} 3$-containing death ligands. Cell Death and Differentiation 15, 1564-1571.

Kwon, O.Y., Hwang, K., Kim, J.A., Kim, K., Kwon, I.C., Song, H.K., and Jeon, H. (2010). Dab1 Binds to Fe65 and Diminishes the Effect of Fe65 or LRP1 on APP Processing. J Cell Biochem 111, 508-519.

Kysenius, K., Muggalla, P., Matlik, K., Arumae, U., and Huttunen, H.J. (2012). PCSK9 regulates neuronal apoptosis by adjusting ApoER2 levels and signaling. Cell Mol Life Sci 69, 19031916.

Lassot, I., Robbins, I., Kristiansen, M., Rahmeh, R., Jaudon, F., Magiera, M., Mora, S., Vanhille, L., Lipkin, A., Pettmann, B., et al. (2010). Trim17, a novel E3 ubiquitin-ligase, initiates neuronal apoptosis. Cell Death and Differentiation 17, 1928-1941.

Lau, K.F., Chan, W.M., Perkinton, M.S., Tudor, E.L., Chang, R.C., Chan, H.Y., McLoughlin, D.M., and Miller, C.C. (2008). Dexras1 interacts with FE65 to regulate FE65-amyloid precursor protein-dependent transcription. J Biol Chem 283, 34728-34737.

Lauwers, E., Jacob, C., and Andre, B. (2009). K63-linked ubiquitin chains as a specific signal for protein sorting into the multivesicular body pathway. J Cell Biol 185, 493-502.

Lee, E.J., Hyun, S., Chun, J., Shin, S.H., and Kang, S.S. (2009a). Ubiquitylation of Fe65 adaptor protein by neuronal precursor cell expressed developmentally down regulated 4-2 (Nedd4-2) via the WW domain interaction with Fe65. Exp Mol Med 41, 555-568.

Lee, H.G., Casadesus, G., Nunomura, A., Zhu, X., Castellani, R.J., Richardson, S.L., Perry, G., Felsher, D.W., Petersen, R.B., and Smith, M.A. (2009b). The Neuronal Expression of MYC Causes a Neurodegenerative Phenotype in a Novel Transgenic Mouse. American Journal of Pathology 174, 891-897.

Lee, J.M., and Bernstein, A. (1995). Apoptosis, Cancer and the P53 Tumor-Suppressor Gene. Cancer Metast Rev 14, 149-161.

Lee, J.T., Wheeler, T.C., Li, L., and Chin, L.S. (2008a). Ubiquitination of alpha-synuclein by 
Siah-1 promotes alpha-synuclein aggregation and apoptotic cell death. Hum Mol Genet 17, 906-917.

Lee, S.H., Choi, J.H., Lee, N., Lee, H.R., Kim, J.I., Yu, N.K., Choi, S.L., Kim, H., and Kaang, B.K. (2008b). Synaptic protein degradation underlies destabilization of retrieved fear memory. Science 319, 1253-1256.

Legube, G., Linares, L.K., Tyteca, S., Caron, C., Scheffner, M., Chevillard-Briet, M., and Trouche, D. (2004). Role of the histone acetyl transferase Tip60 in the p53 pathway. J Biol Chem 279, 44825-44833.

Letai, A., Bassik, M.C., Walensky, L.D., Sorcinelli, M.D., Weiler, S., and Korsmeyer, S.J. (2002). Distinct BH3 domains either sensitize or activate mitochondrial apoptosis, serving as prototype cancer therapeutics. Cancer Cell 2, 183-192.

Li, H., Zhu, H., Xu, C.J., and Yuan, J. (1998). Cleavage of BID by caspase 8 mediates the mitochondrial damage in the Fas pathway of apoptosis. Cell 94, 491-501.

Li, L.Y., Luo, X., and Wang, X. (2001). Endonuclease G is an apoptotic DNase when released from mitochondria. Nature 412, 95-99.

Li, M., Shin, Y.H., Hou, L.F., Huang, X.X., Wei, Z.B., Klann, E., and Zhang, P.M. (2008a). The adaptor protein of the anaphase promoting complex Cdh1 is essential in maintaining replicative lifespan and in learning and memory. Nature Cell Biology 10, 1083-1089.

Li, P., Nijhawan, D., Budihardjo, I., Srinivasula, S.M., Ahmad, M., Alnemri, E.S., and Wang, X. (1997). Cytochrome c and dATP-dependent formation of Apaf-1/caspase-9 complex initiates an apoptotic protease cascade. Cell 91, 479-489.

Li, W., Bengtson, M.H., Ulbrich, A., Matsuda, A., Reddy, V.A., Orth, A., Chanda, S.K., Batalov, S., and Joazeiro, C.A. (2008b). Genome-wide and functional annotation of human E3 ubiquitin ligases identifies MULAN, a mitochondrial E3 that regulates the organelle's dynamics and signaling. PLoS One 3, e1487.

Li, Y., Kong, Y., Zhou, Z., Chen, H., Wang, Z., Hsieh, Y.C., Zhao, D., Zhi, X., Huang, J., Zhang, J., et al. (2013). The HECTD3 E3 ubiquitin ligase facilitates cancer cell survival by promoting K63-linked polyubiquitination of caspase-8. Cell Death Dis 4.

Liew, C.W., Sun, H.Y., Hunter, T., and Day, C.L. (2010). RING domain dimerization is essential for RNF4 function. Biochem J 431, 23-29.

Lim, K.L., and Lim, G.G. (2011). K63-linked ubiquitination and neurodegeneration. Neurobiol Dis 43, 9-16.

Lindsten, T., Ross, A.J., King, A., Zong, W.X., Rathmell, J.C., Shiels, H.A., Ulrich, E., Waymire, K.G., Mahar, P., Frauwirth, K., et al. (2000). The combined functions of proapoptotic Bcl-2 family members bak and bax are essential for normal development of multiple tissues. Mol Cell 6, 1389-1399.

Lipkowitz, S., and Weissman, A.M. (2011). RINGs of good and evil: RING finger ubiquitin ligases at the crossroads of tumour suppression and oncogenesis. Nature Reviews Cancer 11, 629-643.

Liu, X.S., Li, P., Widlak, P., Zou, H., Luo, X., Garrard, W.T., and Wang, X.D. (1998). The 40$\mathrm{kDa}$ subunit of DNA fragmentation factor induces DNA fragmentation and chromatin condensation during apoptosis. P Natl Acad Sci USA 95, 8461-8466.

Liu, Y., Kim, B.O., Kao, C., Jung, C., Dalton, J.T., and He, J.J. (2004). Tip110, the human immunodeficiency virus type 1 (HIV-1) Tat-interacting protein of $110 \mathrm{kDa}$ as a negative regulator of androgen receptor (AR) transcriptional activation. J Biol Chem 279, 2176621773. 
Liu, Y., Lee, M.R., Timani, K., He, J.J., and Broxmeyer, H.E. (2012). Tip110 maintains expression of pluripotent factors in and pluripotency of human embryonic stem cells. Stem Cells Dev 21, 829-833.

Liu, Y., Li, J., Kim, B.O., Pace, B.S., and He, J.J. (2002). HIV-1 Tat protein-mediated transactivation of the HIV-1 long terminal repeat promoter is potentiated by a novel nuclear Tat-interacting protein of $110 \mathrm{kDa}$, Tip110. J Biol Chem 277, 23854-23863.

Liu, Y., Timani, K., Mantel, C., Fan, Y., Hangoc, G., Cooper, S., He, J.J., and Broxmeyer, H.E. (2011). TIP110/p110nrb/SART3/p110 regulation of hematopoiesis through CMYC. Blood $117,5643-5651$.

Liu, Y., Timani, K., Ou, X., Broxmeyer, H.E., and He, J.J. (2013). C-MYC controlled TIP110 protein expression regulates OCT4 mRNA splicing in human embryonic stem cells. Stem Cells Dev 22, 689-694.

Lu, C., Pribanic, S., Debonneville, A., Jiang, C., and Rotin, D. (2007). The PY motif of ENaC, mutated in Liddle syndrome, regulates channel internalization, sorting and mobilization from subapical pool. Traffic 8, 1246-1264.

Lu, Z., and Xu, S. (2006a). ERK1/2 MAP kinases in cell survival and apoptosis. Iubmb Life 58, 621-631.

Lu, Z.M., and Xu, S.C. (2006b). ERK1/2 MAP kinases in cell survival and apoptosis. Iubmb Life $58,621-631$.

Luo, X., Budihardjo, I., Zou, H., Slaughter, C., and Wang, X. (1998). Bid, a Bcl2 interacting protein, mediates cytochrome $\mathrm{c}$ release from mitochondria in response to activation of cell surface death receptors. Cell 94, 481-490.

Ma, Q.H., Futagawa, T., Yang, W.L., Jiang, X.D., Zeng, L., Takeda, Y., Xu, R.X., Bagnard, D., Schachner, M., Furley, A.J., et al. (2008). A TAG1-APP signalling pathway through Fe65 negatively modulates neurogenesis. Nat Cell Biol 10, 283-294.

Macq, A.F., Czech, C., Essalmani, R., Brion, J.P., Maron, A., Mercken, L., Pradier, L., and Octave, J.N. (1998). The long term adenoviral expression of the human amyloid precursor protein shows different secretase activities in rat cortical neurons and astrocytes. Journal of Biological Chemistry 273, 28931-28936.

Magiera, M.M., Mora, S., Mojsa, B., Robbins, I., Lassot, I., and Desagher, S. (2013). Trim17mediated ubiquitination and degradation of Mcl-1 initiate apoptosis in neurons. Cell Death and Differentiation 20, 281-292.

Mahammad, S., Murthy, S.N., Didonna, A., Grin, B., Israeli, E., Perrot, R., Bomont, P., Julien, J.P., Kuczmarski, E., Opal, P., et al. (2013). Giant axonal neuropathy-associated gigaxonin mutations impair intermediate filament protein degradation. J Clin Invest 123, 1964-1975.

Masin, M., Kerschensteiner, D., Dumke, K., Rubio, M.E., and Soto, F. (2006). Fe65 interacts with P2X2 subunits at excitatory synapses and modulates receptor function. J Biol Chem 281, 4100-4108.

Matagne, A., Joris, B., and Frere, J.M. (1991). Anomalous Behavior of a Protein during Sds/Page Corrected by Chemical Modification of Carboxylic Groups. Biochem J 280, 553-556.

Matz, A., Lee, S.J., Schwedhelm-Domeyer, N., Zanini, D., Holubowska, A., Kannan, M., Farnworth, M., Jahn, O., Goepfert, M., and Stegmüller, J. (in press). Regulation of neuronal survival and morphology by the E3 ubiquitin ligase RNF157. Cell Death and Differentiation.

McLoughlin, D.M., and Miller, C.C.J. (1996). The intracellular cytoplasmic domain of the Alzheimer's disease amyloid precursor protein interacts with phosphotyrosine-binding domain proteins in the yeast two-hybrid system. Febs Lett 397, 197-200. 
McLoughlin, D.M., and Miller, C.C.J. (2008). The FE65 proteins and Alzheimer's disease. Journal of Neuroscience Research 86, 744-754.

Minopoli, G., Gargiulo, A., Parisi, S., and Russo, T. (2012). Fe65 matters: New light on an old molecule. Iubmb Life 64, 936-942.

Minopoli, G., Stante, M., Napolitano, F., Telese, F., Aloia, L., De Felice, M., Di Lauro, R., Pacelli, R., Brunetti, A., Zambrano, N., et al. (2007). Essential roles for Fe65, Alzheimer amyloid precursor-binding protein, in the cellular response to DNA damage. J Biol Chem 282, 831835.

Mishra, A., and Jana, N.R. (2008). Regulation of turnover of tumor suppressor p53 and cell growth by E6-AP, a ubiquitin protein ligase mutated in Angelman mental retardation syndrome. Cell Mol Life Sci 65, 656-666.

Morishima, Y., Gotoh, Y., Zieg, J., Barrett, T., Takano, H., Flavell, R., Davis, R.J., Shirasaki, Y., and Greenberg, M.E. (2001). Beta-amyloid induces neuronal apoptosis via a mechanism that involves the c-Jun N-terminal kinase pathway and the induction of Fas ligand. J Neurosci 21, $7551-7560$

Morizane, Y., Honda, R., Fukami, K., and Yasuda, H. (2005). X-linked inhibitor of apoptosis functions as ubiquitin ligase toward mature caspase-9 and cytosolic Smac/DLABLO. J Biochem 137, 125-132.

Morris, M., Maeda, S., Vossel, K., and Mucke, L. (2011). The many faces of tau. Neuron 70, 410-426.

Morris, R. (1984). Developments of a water-maze procedure for studying spatial learning in the rat. J Neurosci Methods 11, 47-60.

Morris, R.G.M., Hagan, J.J., and Rawlins, J.N.P. (1986). Allocentric Spatial-Learning by Hippocampectomized Rats - a Further Test of the Spatial-Mapping and Working Memory Theories of Hippocampal Function. Q J Exp Psychol-B 38, 365-+.

Motoyama, N., Wang, F.P., Roth, K.A., Sawa, H., Nakayama, K., Nakayama, K., Negishi, I., Senju, S., Zhang, Q., Fujii, S., et al. (1995). Massive Cell-Death of Immature Hematopoietic-Cells and Neurons in Bcl-X-Deficient Mice. Science 267, 1506-1510.

Murr, R., Loizou, J.I., Yang, Y.G., Cuenin, C., Li, H., Wang, Z.Q., and Herceg, Z. (2006). Histone acetylation by Trrap-Tip60 modulates loading of repair proteins and repair of DNA doublestrand breaks. Nat Cell Biol 8, 91-99.

Nakamura, Y. (2004). Isolation of p53-target genes and their functional analysis. Cancer Science 95, 7-11.

Nakaya, T., Kawai, T., and Suzuki, T. (2008). Regulation of FE65 nuclear translocation and function by amyloid beta-protein precursor in osmotically stressed cells. J Biol Chem 283, 19119-19131.

Nakaya, T., Kawai, T., and Suzuki, T. (2009). Metabolic stabilization of p53 by FE65 in the nuclear matrix of osmotically stressed cells. FEBS J 276, 6364-6374.

Nakaya, T., and Suzuki, T. (2006). Role of APP phosphorylation in FE65-dependent gene transactivation mediated by AICD. Genes Cells 11, 633-645.

Nakayama, K.I., and Nakayama, K. (2006). Ubiquitin ligases: cell-cycle control and cancer. Nature Reviews Cancer 6, 369-381.

Nateri, A.S., Riera-Sans, L., Da Costa, C., and Behrens, A. (2004). The ubiquitin ligase SCFFbw7 antagonizes apoptotic JNK signaling. Science 303, 1374-1378.

Nathan, J.A., Kim, H.T., Ting, L., Gygi, S.P., and Goldberg, A.L. (2013). Why do cellular proteins linked to K63-polyubiquitin chains not associate with proteasomes? EMBO J 32, 
$552-565$.

Oppenheim, R.W. (1991). Cell-Death during Development of the Nervous-System. Annu Rev Neurosci 14, 453-501.

Pasinelli, P., Belford, M.E., Lennon, N., Bacskai, B.J., Hyman, B.T., Trotti, D., and Brown, R.H., Jr. (2004). Amyotrophic lateral sclerosis-associated SOD1 mutant proteins bind and aggregate with Bcl-2 in spinal cord mitochondria. Neuron 43, 19-30.

Pasinelli, P., Houseweart, M.K., Brown, R.H., Jr., and Cleveland, D.W. (2000). Caspase-1 and -3 are sequentially activated in motor neuron death in $\mathrm{Cu}, \mathrm{Zn}$ superoxide dismutase-mediated familial amyotrophic lateral sclerosis. Proc Natl Acad Sci U S A 97, 13901-13906.

Peixoto, P.M., Lue, J.K., Ryu, S.Y., Wroble, B.N., Sible, J.C., and Kinnally, K.W. (2011). Mitochondrial apoptosis-induced channel (MAC) function triggers a Bax/Bak-dependent bystander effect. Am J Pathol 178, 48-54.

Pelengaris, S., Khan, M., and Evan, G. (2002). c-MYC: more than just a matter of life and death. Nat Rev Cancer 2, 764-776.

Pellow, S., Chopin, P., File, S.E., and Briley, M. (1985). Validation of Open - Closed Arm Entries in an Elevated Plus-Maze as a Measure of Anxiety in the Rat. J Neurosci Meth 14, 149-167.

Pellow, S., and File, S.E. (1986). Anxiolytic and Anxiogenic Drug Effects on Exploratory Activity in an Elevated Plus-Maze - a Novel Test of Anxiety in the Rat. Pharmacol Biochem Be 24, 525-529.

Perciavalle, R.M., Stewart, D.P., Koss, B., Lynch, J., Milasta, S., Bathina, M., Temirov, J., Cleland, M.M., Pelletier, S., Schuetz, J.D., et al. (2012). Anti-apoptotic MCL-1 localizes to the mitochondrial matrix and couples mitochondrial fusion to respiration. Nature Cell Biology 14, 575-+.

Perez, R.G., Soriano, S., Hayes, J.D., Ostaszewski, B., Xia, W.M., Selkoe, D.J., Chen, X.H., Stokin, G.B., and Koo, E.H. (1999). Mutagenesis identifies new signals for beta-amyloid precursor protein endocytosis, turnover, and the generation of secreted fragments, including A beta 42. Journal of Biological Chemistry 274, 18851-18856.

Perkinton, M.S., Standen, C.L., Lau, K.F., Kesavapany, S., Byers, H.L., Ward, M., McLoughlin, D.M., and Miller, C.C. (2004). The c-Abl tyrosine kinase phosphorylates the Fe65 adaptor protein to stimulate Fe65/amyloid precursor protein nuclear signaling. J Biol Chem 279, 22084-22091.

Phan, L.K., Lin, F., LeDuc, C.A., Chung, W.K., and Leibel, R.L. (2002). The mouse mahoganoid coat color mutation disrupts a novel $\mathrm{C} 3 \mathrm{HC} 4 \mathrm{RING}$ domain protein. Journal of Clinical Investigation 110, 1449-1459.

Phillips, R.G., and LeDoux, J.E. (1992). Differential contribution of amygdala and hippocampus to cued and contextual fear conditioning. Behav Neurosci 106, 274-285.

Pick, J.E., Malumbres, M., and Klann, E. (2013). The E3 ligase APC/C-Cdh1 is required for associative fear memory and long-term potentiation in the amygdala of adult mice. Learn Memory 20, 11-20.

Pietrzik, C.U., Yoon, I.S., Jaeger, S., Busse, T., Weggen, S., and Koo, E.H. (2004). FE65 constitutes the functional link between the low-density lipoprotein receptor-related protein and the amyloid precursor protein. J Neurosci 24, 4259-4265.

Pirooznia, S.K., Sarthi, J., Johnson, A.A., Toth, M.S., Chiu, K., Koduri, S., and Elefant, F. (2012). Tip60 HAT activity mediates APP induced lethality and apoptotic cell death in the CNS of a Drosophila Alzheimer's disease model. PLoS One 7, e41776.

Portt, L., Norman, G., Clapp, C., Greenwood, M., and Greenwood, M.T. (2011). Anti-apoptosis 
and cell survival: a review. Biochim Biophys Acta 1813, 238-259.

Poulin, S.P., Dautoff, R., Morris, J.C., Barrett, L.F., Dickerson, B.C., and Initia, A.D.N. (2011). Amygdala atrophy is prominent in early Alzheimer's disease and relates to symptom severity. Psychiat Res-Neuroim 194, 7-13.

Putcha, G.V., Le, S., Frank, S., Besirli, C.G., Clark, K., Chu, B., Alix, S., Youle, R.J., LaMarche, A., Maroney, A.C., et al. (2003). JNK-mediated BIM phosphorylation potentiates BAXdependent apoptosis. Neuron 38, 899-914.

Radyushkin, K., El-Kordi, A., Boretius, S., Castaneda, S., Ronnenberg, A., Reim, K., Bickeboller, H., Frahm, J., Brose, N., and Ehrenreich, H. (2010). Complexin2 null mutation requires a 'second hit' for induction of phenotypic changes relevant to schizophrenia. Genes Brain Behav 9, 592-602.

Reis, D.S., Jarome, T.J., and Helmstetter, F.J. (2013). Memory formation for trace fear conditioning requires ubiquitin-proteasome mediated protein degradation in the prefrontal cortex. Front Behav Neurosci 7, 150.

Reyes-Turcu, F.E., Ventii, K.H., and Wilkinson, K.D. (2009). Regulation and cellular roles of ubiquitin-specific deubiquitinating enzymes. Annu Rev Biochem 78, 363-397.

Reymond, A., Meroni, G., Fantozzi, A., Merla, G., Cairo, S., Luzi, L., Riganelli, D., Zanaria, E., Messali, S., Cainarca, S., et al. (2001). The tripartite motif family identifies cell compartments. EMBO J 20, 2140-2151.

Rigamonti, D., Bauer, J.H., De-Fraja, C., Conti, L., Sipione, S., Sciorati, C., Clementi, E., Hackam, A., Hayden, M.R., Li, Y., et al. (2000). Wild-type huntingtin protects from apoptosis upstream of caspase-3. J Neurosci 20, 3705-3713.

Riley, T., Sontag, E., Chen, P., and Levine, A. (2008). Transcriptional control of human p53regulated genes. Nat Rev Mol Cell Bio 9, 402-412.

Rogan, M.T., Staubli, U.V., and LeDoux, J.E. (1997). Fear conditioning induces associative longterm potentiation in the amygdala. Nature 390, 604-607.

Roos, W.P., and Kaina, B. (2013). DNA damage-induced cell death: from specific DNA lesions to the DNA damage response and apoptosis. Cancer Lett 332, 237-248.

Rotin, D., and Kumar, S. (2009). Physiological functions of the HECT family of ubiquitin ligases. Nat Rev Mol Cell Biol 10, 398-409.

Roullet, P., Mele, A., and Ammassari-Teule, M. (1996). Involvement of glutamatergic and dopaminergic systems in the reactivity of mice to spatial and non-spatial change. Psychopharmacology (Berl) 126, 55-61.

Roy, N., Deveraux, Q.L., Takahashi, R., Salvesen, G.S., and Reed, J.C. (1997). The c-IAP-1 and c-IAP-2 proteins are direct inhibitors of specific caspases. Embo Journal 16, 6914-6925.

Sabo, S.L., Ikin, A.F., Buxbaum, J.D., and Greengard, P. (2001). The Alzheimer amyloid precursor protein (APP) and FE65, an APP-binding protein, regulate cell movement. Journal of Cell Biology 153, 1403-1414.

Sabo, S.L., Ikin, A.F., Buxbaum, J.D., and Greengard, P. (2003). The amyloid precursor protein and its regulatory protein, FE65, in growth cones and synapses in vitro and in vivo. J Neurosci 23, 5407-5415.

Sabo, S.L., Lanier, L.M., Ikin, A.F., Khorkova, O., Sahasrabudhe, S., Greengard, P., and Buxbaum, J.D. (1999). Regulation of beta-amyloid secretion by FE65, an amyloid protein precursor-binding protein. J Biol Chem 274, 7952-7957.

Saeki, Y., Kudo, T., Sone, T., Kikuchi, Y., Yokosawa, H., Toh-e, A., and Tanaka, K. (2009). Lysine 63-linked polyubiquitin chain may serve as a targeting signal for the $26 \mathrm{~S}$ proteasome. 
EMBO J 28, 359-371.

Saito, S., Goodarzi, A.A., Higashimoto, Y., Noda, Y., Lees-Miller, S.P., Appella, E., and Anderson, C.W. (2002). ATM mediates phosphorylation at multiple p53 sites, including Ser(46), in response to ionizing radiation. Journal of Biological Chemistry 277, 1249112494.

Sakahira, H., Enari, M., and Nagata, S. (1998). Cleavage of CAD inhibitor in CAD activation and DNA degradation during apoptosis. Nature 391, 96-99.

Salvesen, G.S., and Duckett, C.S. (2002). IAP proteins: Blocking the road to death's door. Nat Rev Mol Cell Bio 3, 401-410.

Santiard-Baron, D., Langui, D., Delehedde, M., Delatour, B., Schombert, B., Touchet, N., Tremp, G., Paul, M.F., Blanchard, V., Sergeant, N., et al. (2005). Expression of human FE65 in amyloid precursor protein transgenic mice is associated with a reduction in beta-amyloid load. J Neurochem 93, 330-338.

Saudou, F., Finkbeiner, S., Devys, D., and Greenberg, M.E. (1998). Huntingtin acts in the nucleus to induce apoptosis but death does not correlate with the formation of intranuclear inclusions. Cell 95, 55-66.

Scaffidi, C., Fulda, S., Srinivasan, A., Friesen, C., Li, F., Tomaselli, K.J., Debatin, K.M., Krammer, P.H., and Peter, M.E. (1998). Two CD95 (APO-1/Fas) signaling pathways. Embo Journal 17, 1675-1687.

Schwamborn, J.C., Muller, M., Becker, A.H., and Puschel, A.W. (2007). Ubiquitination of the GTPase Rap1B by the ubiquitin ligase Smurf2 is required for the establishment of neuronal polarity. EMBO J 26, 1410-1422.

Shamas-Din, A., Brahmbhatt, H., Leber, B., and Andrews, D.W. (2011). BH3-only proteins: Orchestrators of apoptosis. Bba-Mol Cell Res 1813, 508-520.

Shimura, H., Schwartz, D., Gygi, S.P., and Kosik, K.S. (2004). CHIP-Hsc70 complex ubiquitinates phosphorylated tau and enhances cell survival. J Biol Chem 279, 4869-4876.

Shin, Y.G., Klucken, J., Patterson, C., Hyman, B.T., and McLean, P.J. (2005). The co-chaperone carboxyl terminus of Hsp70-interacting protein (CHIP) mediates alpha-synuclein degradation decisions between proteasomal and lysosomal pathways. Journal of Biological Chemistry 280, 23727-23734.

Shiozaki, E.N., Chai, J., and Shi, Y. (2002). Oligomerization and activation of caspase-9, induced by Apaf-1 CARD. Proc Natl Acad Sci U S A 99, 4197-4202.

Siegel, R.M. (2006). Caspases at the crossroads of immune-cell life and death. Nat Rev Immunol 6, 308-317.

Silvius, D., Pitstick, R., Ahn, M., Meishery, D., Oehler, A., Barsh, G.S., DeArmond, S.J., Carlson, G.A., and Gunn, T.M. (2013). Levels of the Mahogunin Ring Finger 1 E3 ubiquitin ligase do not influence prion disease. PLoS One 8, e55575.

Simon, P., Dupuis, R., and Costentin, J. (1994). Thigmotaxis as an Index of Anxiety in Mice Influence of Dopaminergic Transmissions. Behav Brain Res 61, 59-64.

Slee, E.A., Harte, M.T., Kluck, R.M., Wolf, B.B., Casiano, C.A., Newmeyer, D.D., Wang, H.G., Reed, J.C., Nicholson, D.W., Alnemri, E.S., et al. (1999). Ordering the cytochrome cinitiated caspase cascade: hierarchical activation of caspases-2, $-3,-6,-7,-8$, and -10 in a caspase-9-dependent manner. J Cell Biol 144, 281-292.

Song, E.J., Werner, S.L., Neubauer, J., Stegmeier, F., Aspden, J., Rio, D., Harper, J.W., Elledge, S.J., Kirschner, M.W., and Rape, M. (2010). The Prp19 complex and the Usp4(Sart3) deubiquitinating enzyme control reversible ubiquitination at the spliceosome. Gene Dev 24, 
1434-1447.

Song, M.S., Salmena, L., and Pandolfi, P.P. (2012). The functions and regulation of the PTEN tumour suppressor. Nat Rev Mol Cell Biol 13, 283-296.

Squatrito, M., Gorrini, C., and Amati, B. (2006). Tip60 in DNA damage response and growth control: many tricks in one HAT. Trends Cell Biol 16, 433-442.

Srinivasula, S.M., Ahmad, M., Fernandes-Alnemri, T., and Alnemri, E.S. (1998). Autoactivation of procaspase- 9 by Apaf-1-mediated oligomerization. Mol Cell 1, 949-957.

Stante, M., Minopoli, G., Passaro, F., Raia, M., Vecchio, L.D., and Russo, T. (2009). Fe65 is required for Tip60-directed histone $\mathrm{H} 4$ acetylation at DNA strand breaks. Proc Natl Acad Sci U S A 106, 5093-5098.

Staropoli, J.F., McDermott, C., Martinat, C., Schulman, B., Demireva, E., and Abeliovich, A. (2003). Parkin is a component of an SCF-like ubiquitin ligase complex and protects postmitotic neurons from kainate excitotoxicity. Neuron 37, 735-749.

Sun, Y.L., Jiang, X.F., Chen, S.J., Fernandes, N., and Price, B.D. (2005). A role for the Tip60 histone acetyltransferase in the acetylation and activation of ATM. P Natl Acad Sci USA 102, 13182-13187.

Susin, S.A., Lorenzo, H.K., Zamzami, N., Marzo, I., Snow, B.E., Brothers, G.M., Mangion, J., Jacotot, E., Costantini, P., Loeffler, M., et al. (1999). Molecular characterization of mitochondrial apoptosis-inducing factor. Nature 397, 441-446.

Suzuki, Y., Imai, Y., Nakayama, H., Takahashi, K., Takio, K., and Takahashi, R. (2001a). A serine protease, HtrA2, is released from the mitochondria and interacts with XIAP, inducing cell death. Molecular Cell 8, 613-621.

Suzuki, Y., Nakabayashi, Y., and Takahashi, R. (2001b). Ubiquitin-protein ligase activity of Xlinked inhibitor of apoptosis protein promotes proteasomal degradation of caspase-3 and enhances its anti-apoptotic effect in Fas-induced cell death. Proc Natl Acad Sci U S A 98, $8662-8667$.

Swerdlow, N.R., Braff, D.L., and Geyer, M.A. (2000). Animal models of deficient sensorimotor gating: what we know, what we think we know, and what we hope to know soon. Behav Pharmacol 11, 185-204.

Takuma, H., Tomiyama, T., Kuida, K., and Mori, H. (2004). Amyloid beta peptide-induced cerebral neuronal loss is mediated by caspase-3 in vivo. J Neuropathol Exp Neurol 63, 255261.

Tan, D.P., Liu, Q.Y., Koshiya, N., Gu, H., and Alkon, D. (2006). Enhancement of long-term memory retention and short-term synaptic plasticity in cbl-b null mice. Proc Natl Acad Sci U S A 103, 5125-5130.

Tanahashi, H., and Tabira, T. (1999). Molecular cloning of human Fe65L2 and its interaction with the Alzheimer's beta-amyloid precursor protein. Neuroscience Letters 261, 143-146.

Tang, Y., Luo, J., Zhang, W., and Gu, W. (2006). Tip60-dependent acetylation of p53 modulates the decision between cell-cycle arrest and apoptosis. Mol Cell 24, 827-839.

Tao, W., and Levine, A.J. (1999). Nucleocytoplasmic shuttling of oncoprotein Hdm2 is required for Hdm2-mediated degradation of p53. Proc Natl Acad Sci U S A 96, 3077-3080.

Tofaris, G.K., Kim, H.T., Hourez, R., Jung, J.W., Kim, K.P., and Goldberg, A.L. (2011). Ubiquitin ligase Nedd4 promotes alpha-synuclein degradation by the endosomal-lysosomal pathway. Proc Natl Acad Sci U S A 108, 17004-17009.

Trede, N.S., Medenbach, J., Damianov, A., Hung, L.H., Weber, G.J., Paw, B.H., Zhou, Y., Hersey, C., Zapata, A., Keefe, M., et al. (2007). Network of coregulated spliceosome components 
revealed by zebrafish mutant in recycling factor p110. Proc Natl Acad Sci U S A 104, 66086613.

Treit, D., and Fundytus, M. (1988). Thigmotaxis as a Test for Anxiolytic Activity in Rats. Pharmacol Biochem Be 31, 959-962.

Treit, D., Menard, J., and Royan, C. (1993). Anxiogenic Stimuli in the Elevated Plus-Maze. Pharmacol Biochem Be 44, 463-469.

Trommsdorff, M., Borg, J.P., Margolis, B., and Herz, J. (1998). Interaction of cytosolic adaptor proteins with neuronal apolipoprotein E receptors and the amyloid precursor protein. J Biol Chem 273, 33556-33560.

Tyteca, S., Vandromme, M., Legube, G., Chevillard-Briet, M., and Trouche, D. (2006). Tip60 and p400 are both required for UV-induced apoptosis but play antagonistic roles in cell cycle progression. EMBO J 25, 1680-1689.

Urushitani, M., Kurisu, J., Tateno, M., Hatakeyama, S., Nakayama, K., Kato, S., and Takahashi, R. (2004). CHIP promotes proteasomal degradation of familial ALS-linked mutant SOD1 by ubiquitinating Hsp/Hsc70. J Neurochem 90, 231-244.

van Loo, G., Schotte, P., van Gurp, M., Demol, H., Hoorelbeke, B., Gevaert, K., Rodriguez, I., Ruiz-Carrillo, A., Vandekerckhove, J., Declercq, W., et al. (2001). Endonuclease G: a mitochondrial protein released in apoptosis and involved in caspase-independent DNA degradation. Cell Death and Differentiation 8, 1136-1142.

Vazquez, M.C., Vargas, L.M., Inestrosa, N.C., and Alvarez, A.R. (2009). c-Abl modulates AICD dependent cellular responses: transcriptional induction and apoptosis. J Cell Physiol 220, 136-143.

Verhagen, A.M., Ekert, P.G., Pakusch, M., Silke, J., Connolly, L.M., Reid, G.E., Moritz, R.L., Simpson, R.J., and Vaux, D.L. (2000). Identification of DIABLO, a mammalian protein that promotes apoptosis by binding to and antagonizing IAP proteins. Cell 102, 43-53.

Verhagen, A.M., Silke, J., Ekert, P.G., Pakusch, M., Kaufmann, H., Connolly, L.M., Day, C.L., Tikoo, A., Burke, R., Wrobel, C., et al. (2002). HtrA2 promotes cell death through its serine protease activity and its ability to antagonize inhibitor of apoptosis proteins. Journal of Biological Chemistry 277, 445-454.

Villunger, A., Michalak, E.M., Coultas, L., Mullauer, F., Bock, G., Ausserlechner, M.J., Adams, J.M., and Strasser, A. (2003). p53- and drug-induced apoptotic responses mediated by BH3only proteins puma and noxa. Science $302,1036-1038$.

von Rotz, R.C., Kohli, B.M., Bosset, J., Meier, M., Suzuki, T., Nitsch, R.M., and Konietzko, U. (2004). The APP intracellular domain forms nuclear multiprotein complexes and regulates the transcription of its own precursor. J Cell Sci 117, 4435-4448.

Vucic, D., Dixit, V.M., and Wertz, I.E. (2011). Ubiquitylation in apoptosis: a post-translational modification at the edge of life and death. Nat Rev Mol Cell Biol 12, 439-452.

Wang, B., Hu, Q., Hearn, M.G., Shimizu, K., Ware, C.B., Liggitt, D.H., Jin, L.W., Cool, B.H., Storm, D.R., and Martin, G.M. (2004). Isoform-specific knockout of FE65 leads to impaired learning and memory. J Neurosci Res 75, 12-24.

Wang, H.R., Zhang, Y., Ozdamar, B., Ogunjimi, A.A., Alexandrova, E., Thomsen, G.H., and Wrana, J.L. (2003). Regulation of cell polarity and protrusion formation by targeting RhoA for degradation. Science 302, 1775-1779.

Wang, S.H., and Morris, R.G. (2010). Hippocampal-neocortical interactions in memory formation, consolidation, and reconsolidation. Annu Rev Psychol 61, 49-79, C41-44.

Wang, Y., Zhang, M., Moon, C., Hu, Q., Wang, B., Martin, G., Sun, Z., and Wang, H. (2009). The 
APP-interacting protein FE65 is required for hippocampus-dependent learning and longterm potentiation. Learn Mem 16, 537-544.

Watanabe, M., Dykes-Hoberg, M., Culotta, V.C., Price, D.L., Wong, P.C., and Rothstein, J.D. (2001). Histological evidence of protein aggregation in mutant SOD1 transgenic mice and in amyotrophic lateral sclerosis neural tissues. Neurobiol Dis 8, 933-941.

Wei, M.C., Lindsten, T., Mootha, V.K., Weiler, S., Gross, A., Ashiya, M., Thompson, C.B., and Korsmeyer, S.J. (2000). tBID, a membrane-targeted death ligand, oligomerizes BAK to release cytochrome c. Genes Dev 14, 2060-2071.

Wei, M.C., Zong, W.X., Cheng, E.H.Y., Lindsten, T., Panoutsakopoulou, V., Ross, A.J., Roth, K.A., MacCregor, G.R., Thompson, C.B., and Korsmeyer, S.J. (2001). Proapoptotic BAX and BAK: A requisite gateway to mitochondrial dysfunction and death. Science 292, 727730 .

Weissman, A.M., Shabek, N., and Ciechanover, A. (2011). The predator becomes the prey: regulating the ubiquitin system by ubiquitylation and degradation. Nat Rev Mol Cell Biol 12, 605-620.

Welcker, M., Orian, A., Jin, J.P., Grim, J.A., Harper, J.W., Eisenman, R.N., and Clurman, B.E. (2004). The Fbw7 tumor suppressor regulates glycogen synthase kinase 3 phosphorylationdependent c-Myc protein degradation. P Natl Acad Sci USA 101, 9085-9090.

Whatley, B.R., Li, L., and Chin, L.S. (2008). The ubiquitin-proteasome system in spongiform degenerative disorders. Biochim Biophys Acta 1782, 700-712.

White, F.A., Keller-Peck, C.R., Knudson, C.M., Korsmeyer, S.J., and Snider, W.D. (1998). Widespread elimination of naturally occurring neuronal death in Bax-deficient mice. J Neurosci 18, 1428-1439.

Willis, S.N., Chen, L., Dewson, G., Wei, A., Naik, E., Fletcher, J.I., Adams, J.M., and Huang, D.C.S. (2005). Proapoptotic Bak is sequestered by Mcl-1 and Bcl-x(L), but not Bcl-2, until displaced by BH3-only proteins. Gene Dev 19, 1294-1305.

Witting, A., Muller, P., Herrmann, A., Kettenmann, H., and Nolte, C. (2000). Phagocytic clearance of apoptotic neurons by Microglia/Brain macrophages in vitro: involvement of lectin-, integrin-, and phosphatidylserine-mediated recognition. J Neurochem 75, 1060-1070.

Xu, L., Chen, Y., Song, Q., Xu, D., Wang, Y., and Ma, D. (2009a). PDCD5 interacts with Tip60 and functions as a cooperator in acetyltransferase activity and DNA damage-induced apoptosis. Neoplasia 11, 345-354.

Xu, P., Duong, D.M., Seyfried, N.T., Cheng, D., Xie, Y., Robert, J., Rush, J., Hochstrasser, M., Finley, D., and Peng, J. (2009b). Quantitative proteomics reveals the function of unconventional ubiquitin chains in proteasomal degradation. Cell 137, 133-145.

Yamamoto, T., and Horikoshi, M. (1997). Novel substrate specificity of the histone acetyltransferase activity of HIV-1-Tat interactive protein Tip60. J Biol Chem 272, 3059530598.

Yang, Y., Fang, S.Y., Jensen, J.P., Weissman, A.M., and Ashwell, J.D. (2000). Ubiquitin protein ligase activity of IAPs and their degradation in proteasomes in response to apoptotic stimuli. Science 288, 874-877.

Yao, I., Takao, K., Miyakawa, T., Ito, S., and Setou, M. (2011). Synaptic E3 Ligase SCRAPPER in Contextual Fear Conditioning: Extensive Behavioral Phenotyping of Scrapper Heterozygote and Overexpressing Mutant Mice. PLoS One 6.

Yasuda, T., Nakata, Y., and Mochizuki, H. (2013). alpha-Synuclein and neuronal cell death. Mol Neurobiol 47, 466-483. 
Ye, Y.H., and Rape, M. (2009). Building ubiquitin chains: E2 enzymes at work. Nat Rev Mol Cell Bio 10, 755-764.

Yip, K.W., and Reed, J.C. (2008). Bcl-2 family proteins and cancer. Oncogene 27, 6398-6406.

Yoshida, H., Kong, Y.Y., Yoshida, R., Elia, A.J., Hakem, A., Hakem, R., Penninger, J.M., and Mak, T.W. (1998). Apaf1 is required for mitochondrial pathways of apoptosis and brain development. Cell 94, 739-750.

Zambrano, N., Bimonte, M., Arbucci, S., Gianni, D., Russo, T., and Bazzicalupo, P. (2002). feh-1 and apl-1, the Caenorhabditis elegans orthologues of mammalian Fe65 and beta-amyloid precursor protein genes, are involved in the same pathway that controls nematode pharyngeal pumping. J Cell Sci 115, 1411-1422.

Zhang, Y., McLaughlin, R., Goodyer, C., and LeBlanc, A. (2002). Selective cytotoxicity of intracellular amyloid beta peptide1-42 through p53 and Bax in cultured primary human neurons. J Cell Biol 156, 519-529.

Zhao, H., Sapolsky, R.M., and Steinberg, G.K. (2006). Phosphoinositide-3-kinase/akt survival signal pathways are implicated in neuronal survival after stroke. Mol Neurobiol 34, 249-270.

Zhao, W., Liu, Y., Timani, K.A., and He, J.J. (2014). Tip110 protein binds to unphosphorylated RNA polymerase II and promotes its phosphorylation and HIV-1 long terminal repeat transcription. J Biol Chem 289, 190-202.

Zhong, Q., Gao, W.H., Du, F.H., and Wang, X.D. (2005). Mule/ARF-BP1, a BH3-only E3 ubiquitin ligase, catalyzes the polyubiquitination of Mcl-1 and regulates apoptosis. Cell 121, 1085-1095.

Zou, H., Henzel, W.J., Liu, X., Lutschg, A., and Wang, X. (1997). Apaf-1, a human protein homologous to $\mathrm{C}$. elegans CED-4, participates in cytochrome c-dependent activation of caspase-3. Cell 90, 405-413.

Zou, H., Li, Y., Liu, X., and Wang, X. (1999). An APAF-1.cytochrome c multimeric complex is a functional apoptosome that activates procaspase-9. J Biol Chem 274, 11549-11556.

Zucchelli, S., Marcuzzi, F., Codrich, M., Agostoni, E., Vilotti, S., Biagioli, M., Pinto, M., Carnemolla, A., Santoro, C., Gustincich, S., et al. (2011). Tumor necrosis factor receptorassociated factor 6 (TRAF6) associates with huntingtin protein and promotes its atypical ubiquitination to enhance aggregate formation. J Biol Chem 286, 25108-25117. 